San Jose State University

SJSU ScholarWorks

Master's Theses

Master's Theses and Graduate Research

Fall 2014

\title{
The Role of Lithology in Glacial Valley Cross-Sectional Shape in Sierra Nevada, California
}

Kaai Jensen

San Jose State University

Follow this and additional works at: https://scholarworks.sjsu.edu/etd_theses

\section{Recommended Citation}

Jensen, Kaai, "The Role of Lithology in Glacial Valley Cross-Sectional Shape in Sierra Nevada, California" (2014). Master's Theses. 4497.

DOI: https://doi.org/10.31979/etd.pkkt-wf95

https://scholarworks.sjsu.edu/etd_theses/4497

This Thesis is brought to you for free and open access by the Master's Theses and Graduate Research at SJSU ScholarWorks. It has been accepted for inclusion in Master's Theses by an authorized administrator of SJSU ScholarWorks. For more information, please contact scholarworks@sjsu.edu. 


\title{
THE ROLE OF LITHOLOGY IN GLACIAL VALLEY CROSS-SECTIONAL SHAPE IN SIERRA NEVADA, CALIFORNIA
}

\author{
A Thesis \\ Presented to \\ The Faculty of the Department of Geology \\ San Jose State University \\ In Partial Fulfillment \\ of the Requirements for the Degree \\ Master of Science
}

by

Ka'ai Jensen

December 2014 
(C) 2014

Ka'ai Jensen

ALL RIGHTS RESERVED 
The Designated Thesis Committee Approves the Thesis Titled THE ROLE OF LITHOLOGY IN GLACIAL VALLEY CROSS-SECTIONAL PROFILE IN SIERRA NEVADA, CALIFORNIA by

Ka'ai Jensen

APPROVED FOR THE DEPARTMENT OF GEOLOGY SAN JOSE STATE UNIVERSITY

December 2014
Dr. Emmanuel Gabet
Department of Geology
Dr. Jonathan Miller
Department of Geology
Dr. Paula Messina
Department of Geology
Dr. Luis Bonachea
Department of Biology 


\section{ABSTRACT \\ THE ROLE OF LITHOLOGY IN GLACIAL VALLEY CROSS-SECTIONAL PROFILE IN SIERRA NEVADA, CALIFORNIA}

It is commonly inferred that alpine glaciers transform fluvial valleys from $\mathrm{V}$ to $\mathrm{U}$ shaped. It has been suggested that, under certain circumstances, glaciers are unable to modify V-shaped valleys and that lithology may play an important role in a glacier's ability to effectively erode bedrock. To test this hypothesis, six glaciated valleys with both U and V-shaped cross sections were chosen in the Sierra Nevada (CA) to examine how lithological properties might affect patterns of glacial erosion: Tenaya Canyon, Tuolumne River, Little Yosemite Valley, the San Joaquin River, the north fork of the King River, and Deadman Canyon. Valley profiles were

fitted with the power law, $y=a x^{b}$, where the exponent $b$ represents the shape of the cross section. Rock mass strength (RMS) values for these sites were determined by field inspection. No clear relationship was found between RMS and valley shape. Although, there was a weak association between measured joint orientation and valley axis in Little Yosemite Valley, the North Fork of the King River, and the San Joaquin, the two variables appear unrelated in Tenaya Creek, Tuolumne River, and Deadman Canyon. These results propose that in the Sierra Nevada granitic batholith, patterns of glacial erosion in granitic rock are not dependent on rock mass strength (as typically measured), while joint orientation may play a minor role. 


\section{ACKNOWLEDGMENTS}

This project has had many contributors in the last two and a half years, each devoting their time and knowledge to the completion of a venture that has been a

long time coming. I would like to first acknowledge my advisor, Manny Gabet, who was the inspiration behind the subject matter, my heartfelt thanks for his continued support, encouragement, insight, and patience. I will treasure his firm belief in the usage of a thesaurus when writing. Behind any good writer is a good editor, and I would like to recognize Tim Carlson and Manny Gabet for their continued efforts in reading and improving this work.

I would also like to recognize the assistance of my committee and the contribution of their knowledge in the writing of this paper. I am indebted, as always, to my mom and brother who have continued to faithfully support me in all of my endeavors. Lastly, my sincere appreciation to all of my resolute field assistants: Tim and Dave Carlson, Gregg Armstrong, and Robert Neusel for braving an often difficult, yet rewarding, field season. 


\section{Table of Contents}

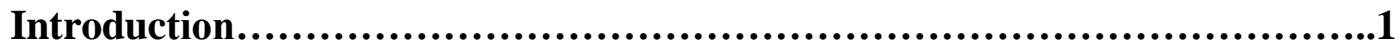

Materials and Methods......................................................4

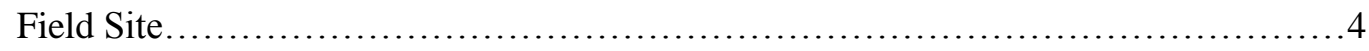

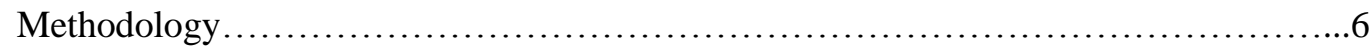

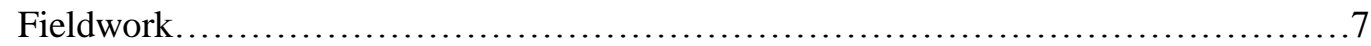

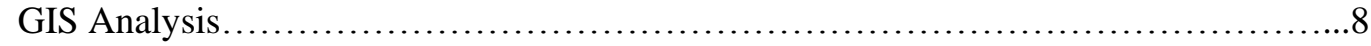

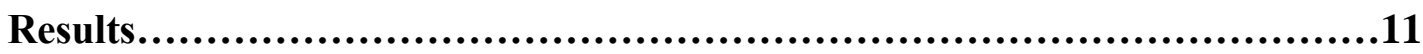

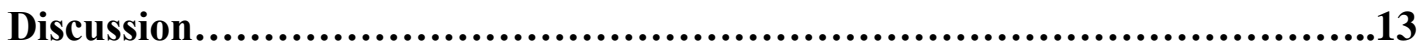

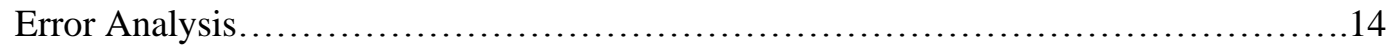

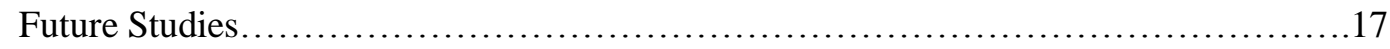

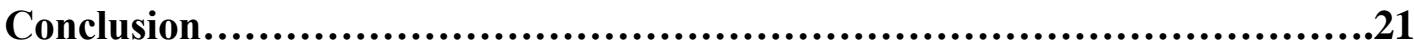




\section{List of Figures}

Figure 1. Cross sectional transect of Tuolumne River Valley, Yosemite National Park, CA ....... 22

Figure 2. Map of California showing the locations of the six field sites ..................................... 23

Figure 3. Ten meter DEM of Tuolumne River Valley, Yosemite National Park, CA ................... 24

Figure 4. Ten meter DEM of Tenaya Canyon, Yosemite National Park, CA............................... 25

Figure 5. Ten meter DEM of Little Yosemite Valley, Yosemite National Park, CA ................... 26

Figure 6. Ten meter DEM of the San Joaquin River, Sequoia National Forest, CA..................... 27

Figure 7. Ten meter DEM of the North Fork King River, Sequoia National Forest, CA ............. 28

Figure 8. Ten meter DEM of Deadman Canyon, Kings Canyon National Park, CA..................... 29

Figure 9. Example of a $\mathrm{U}$ and a V-shaped valley from Tuolumne River Valley ........................... 30

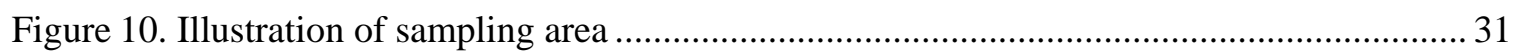

Figure 11.Matlab curve fit for half a U-shaped transect from Tuolumne River Valley ................. 32

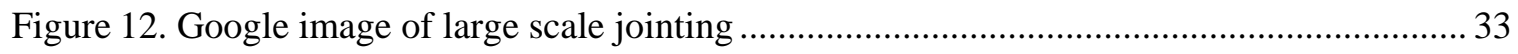

Figure 13. Graphs showing relationship between RMS and valley shape for all six field sites .... 34

Figure 14. Graph showing median joint spacing for all six Sierra Nevada valleys ....................... 35

Figure 15. Equal area projection stereonets for all six field areas ............................................. 36

Figure 16. Photo illustrating joint bounded blocks from Tenaya Canyon .................................... 37

Figure 17. Plot showing the mode of the delta strike vs. average $b$ for 19 reaches from the six

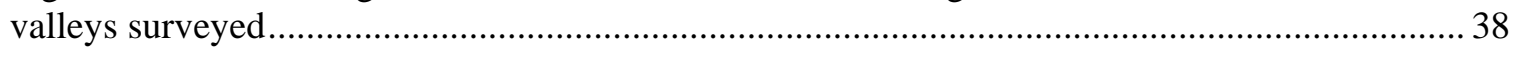

Figure 18. Percent frequency histogram for the mode of delta strike orientations ....................... 39

Figure 19. Photo of perpendicular microfractures from Tenaya Canyon...................................... 40

Figure 20. Photo illustrating in situ blocks with fracture spacing greater than 1 meter................. 41 


\section{List of Tables}

Table 1. Previous glaciations from the last 3 million years from the Sierra Nevada.......42

Table 2. Rock mass classification chart from Selby (1980).........................................43 


\section{Introduction}

Horton (1945) was one of the first to examine the erosional development of fluvial systems from a geomorphologic perspective utilizing the idea that river systems develop V-shaped valleys. V-shaped valley morphology has been used to determine the fluvial origin of river valleys in Alberta, Canada (Smith and Fisher, 1993). Numerous geomorphological studies of Martian valleys have also used Vshaped cross-sections as evidence for the existence of surface water processes on another planet (e.g. Brakenridge et al., 1985; Gulick and Baker, 1990; Baker, 2001). The idea that U-shaped cross- sectional profiles represent a typical glacial valley was introduced by Matthes (1930). This morphology has continued to be considered the classic indicator for glacially carved valleys (e.g. Potter, 1976; Harbor, 1995; MacGregor et al., 2000) and Yosemite Valley in the Sierra Nevada, California, is recognized as one of the iconic symbols representing this classic glacial U-shape. Large alpine glaciers last occupied the Sierra Nevada mountain range approximately 18,000 years ago (Birkland, 1964; Bateman and Wahrhaftig, 1966; James, 2003; Gillespie and Zehfuss, 2004). Matthes (1930) was the first to make a thorough inspection of the extent and morphology of presumed glacial erosion in the central and southern Sierra Nevada. Matthes' initial field work began in Yosemite Valley and expanded to the San Joaquin, Kern, and Kaweah river basins (Matthes 1960, 1965). Although Matthes classified much of Yosemite Valley as U-shaped, he noted areas above Bridal Veil Falls and sections along the Tuolumne River valley as being 
V-shaped (Figure 1) and suggested that lithological characteristics, such as the extent of jointing, may play an important role in a glacier's ability to effectively erode bedrock.

Boulton (1979) was one of the earliest works to compare the formation of erosional landforms in two different lithologies: lithified and unlithified sediment. By examining the erosional processes that formed these landforms beneath active glaciers, Boulton (1979) suggested that quarrying was the dominant erosional process in lithified sediment and abrasion was the principal process in eroding unlithified sediment. Research into bedrock control on the subglacial formation of rock steps in Camp Glacier, Oksfjordjokelen (Norway) found that the basal shear stress produced by a flowing glacier was sufficient to remove any joint-bounded bedrock blocks, indicating that glacial erosion by quarrying is an important process in fractured rock (Rea, 1994). Hay (1943) speculated that the Pennine valley in northern England was both $\mathrm{V}$ and U-shaped based on the distribution of joint spacing in the rock. He believed that closer joint spacing resulted in more Ushaped valleys. A comparison of glacial valleys in highly jointed metamorphic rocks and non-jointed plutonic rocks in the Southern Alps, New Zealand concluded that the presence of joints and their spacing, orientation, and width contributed to the formation of broader, flatter glacial troughs (Augustinus, 1992). Augustinus (1995) expanded on his 1992 study and examined how rock mass strength (RMS) and a rock's in situ stress-field controlled the direction and extent of slope 
modification in glacial valleys. He concluded that broader, shallower valleys developed in areas with low RMS on valley slopes. Brook et al. (2004) compared cross-sectional profiles of glacial valleys in igneous and metamorphic rocks with RMS and rock intact strength in the Scottish Highlands and NW Iceland. Brook et al.'s (2004) study concluded that rock intact strength had a negligible effect on valley morphology and that joint spacing, joint orientation, and joint width were the most effective at explaining cross-section profile morphology in glacial valleys.

Krabbendam and Glasser (2011) analyzed the formation of glacial features, (e.g. whale backs) through the processes of quarrying and abrasion between intact versus fractured sandstone and quartzite in northwest Scotland. They concluded that quarrying is the dominant erosional method in hard rock with dense joint spacing, while softer rocks with greater joint spacing are eroded by abrasion. Cosmogenic radionuclide $(\mathrm{CRN})$ concentrations in bedrock were measured to determine past glacial erosion rates within the Tuolumne River basin in Yosemite National Park and compared with joint density for two units of the Tuolumne Intrusive Series and compared to past erosion rates (Duhnforth et al., 2010). Duhnforth et al. (2010) concluded that when fracture spacing is less than $1 \mathrm{~m}$, quarrying of the bedrock is the dominant process, whereas in less fractured bedrock, abrasion dominates. In a survey of an Alaskan glacial lake, Loso et al. (2004) discovered that coarse material carried as bedload is often underrepresented in most estimates of total sediment yield. Their measurements showed that bedload material comprised $80-85 \%$ of 
the total load backing the interpretation that quarrying is an important process in glacial erosion. Experiments by Sklar and Dietrich (2001) showed that, under fluvial conditions, granite is more resistant to abrasion than most other rock lithologies, supporting the idea that fractures are necessary for efficient erosion of granitic rock.

I hypothesized that rock hardness, joint density, and joint orientation strongly influence the cross-sectional shape of glacially eroded valleys in the Sierra Nevada batholith. Duhnforth et al. (2010) showed that where abrasion is the dominant process, erosion rates are slow, and in valleys where quarrying is the dominant process, erosion rates are much faster. Therefore, regions with abrasion-dominant erosion should experience less valley modification and exhibit a V-shaped crosssectional profile, while regions with quarrying-dominant erosion should display Ushaped profiles.

\section{Materials and Methods}

\section{Field Site}

The Sierra Nevada mountain range with its varied glaciated-valley cross-sections presents a unique opportunity to study the effects of lithology on glacial erosional processes. Seven major glacial advances in the Sierra Nevada over the last $\sim 3$ million years are recognized by Gillespie and Zehfuss, (2004) (Table 1). Based on glacial maps from Moore and Mack (2008), Alpha et al. (1987), and Matthes (1960, 1965), six previously glaciated regions composed of granitoids within the 
Sierra Nevada were selected for this study: the Tuolumne River Canyon, Tenaya Canyon, Little Yosemite Valley, the San Joaquin River Canyon, the Canyon of the North Fork of the Kings River and Deadman Canyon (Figure 2). The Sierra Nevada batholith, comprised of Mesozoic-aged plutonic rocks, forms the majority of the Sierra Nevada mountain range (Bateman and Wahrhaftig, 1966). The Tuolumne River site is the most heterogeneous of the six sites consisting of Cretaceous leucogranite of Ten Lakes, equigranular facies of the Half Dome granodiorite, the Johnson granite porphyry, the coarse grained Mount Hoffman granodiorite, and a Tertiary trachybasalt plug (Bateman et al., 1983). Tenaya Canyon is formed principally of the medium grained Cretaceous equigranular facies of the Half Dome granodiorite (Bateman et al., 1983). Half Dome quartz monzonite and medium grained equigranular facies of Half Dome granodiorite constitutes the majority of Little Yosemite Valley (Bateman et al., 1983). The Mount Givens granodiorite forms the bulk of the Middle Fork of the San Joaquin River valley (Bateman et al., 1971; Huber and Rinehart, 1965). The North Fork of the King River valley runs primarily through Mount Given granodiorite with patches of Finger Peak felsic quartz monzonite at its eastern end (Bateman, 1965). Deadman Canyon is made up entirely of Sugarloaf granodiorite with the exception of small pockets of Quaternary alluvium scattered throughout (Moore and Sisson, 1978). 


\section{Methodology}

Using 10 m DEMs (digital elevation models) (Figures 3-8) in ArcGIS, I visually confirmed that each valley has profiles that span the range from $\mathrm{V}$ to U-shape (Figures 9A and 9B). The valleys were divided into transects which extended from the base of the valley to the upper limits of glacial erosion $(\sim 200 \mathrm{~m})$ or the top of the valley, making sure that each transect was drawn perpendicular to elevation contours. The maximum elevation over which effective glacial erosion occurred was selected at two hundred meters (Matthes, 1930; Moscicki, et al., 2006). Each transect was then divided into a series of circles with a radius of $\sim 1.3 \mathrm{~m}$. The circles continued from the base of the valley proceeding up the valley slope perpendicular to glacial flow with subsequent circles located $\sim 10 \mathrm{~m}$ above the previous one (Figure 10). Measurements of rock intact strength, rock weathering, joint orientation, joint spacing, joint width, joint continuity, and outflow of groundwater were taken within the area of each circle. This procedure was repeated for approximately 10 transects along each of the longitudinal profiles of Tuolumne River valley, Tenaya Canyon, Little Yosemite Valley, the San Joaquin River valley, the North Fork of the King River Valley and Deadman Canyon. An attempt was made to use transects from valley walls that were directly opposite each other in order to delineate the cross-sectional shape, but due to accessibility problems in many areas, this was often not feasible. 


\section{Fieldwork}

An N-type Schmidt hammer was used to determine the rock intact strength. This instrument is extremely sensitive to discontinuities in a rock and so should not be used on rock that is fissile, laminated or closely foliated. Goudie (2006) recommends that the Schmidt hammer be used on large blocks of rock weighing more than $25 \mathrm{~kg}$ avoiding surface irregularities as they may influence the results and so tests should be done on a rock surface that is as free from defects as possible. The effectiveness of the Schmidt hammer is also improved when it is used parallel to the force of gravity (Goudie, 2006), although in instances where measurements cannot be taken perpendicular to the rock face a correction can be made using the Schmidt hammer manual.

Following Selby's (1980) RMS classification chart, each of the measured parameters was assigned a range of values that were grouped into five classes. This range of values was then assigned an "r" rating reflecting the strength of the rock. For example, an average spacing between measured joints of $0.6 \mathrm{~m}$ falls within the range of $0.3-1 \mathrm{~m}$ and so was given an " $\mathrm{r}$ " rating of 21 , indicating a rock of moderate strength (Table 2). The " $r$ " rating number for rock intact strength, rock weathering, joint orientation, joint spacing, joint width, joint continuity, and outflow of groundwater were then summed to determine the total RMS value ranging from 1 to 100 . A very weak rock has an RMS value of $<26$ and a very strong rock has an RMS value of 91-100. 
For the purpose of this research, a joint is defined as all discontinuities throughout the bedrock (Selby, 1980; Palmstrom, 2005). The orientation of the fracture is the attitude of the joint in space as defined by its strike and dip direction (Gumede and Stacey, 2007). Spacing is the perpendicular distance between two joints (Palmstrom, 2005), and joint width is the width of the aperture between two adjacent joint walls (Selby, 1980). Any joint that terminates at another joint is considered discontinuous (Palmstrom, 1995), all others are continuous. Joint infill is any material that is of dissimilar composition to that of the rock type in which the joint is found (Selby, 1980). Following Palmstrom (2005) and Selby (1980) joints less than $5 \mathrm{~mm}$ in length (microjoints) were not considered as they do not contribute significantly to the weakening of rock.

\section{GIS Analysis}

A quantitative analysis of valley cross-sectional morphology adopted by some (e.g., Brook et al., 2004; Yingkui et al., 2001; Augustinus 1995; Hirano and Aniya, 1988; Graf, 1970) uses the power law,

$y=a x^{b}$

where $x$ is the horizontal distance (in meters) measured from the center of valley, $y$ is the height (in meters) from the center to that point, and $a$ and $b$ are constants with increases in $b$ toward 2.0 reflecting a broader $\mathrm{U}$-shaped valley. A $b$ value of 1.0 indicates a narrow V- shaped valley (Brook et al. 2004; Yingkui et al. 2001). A MATLAB (2012) program was used to calculate the value of $b$ from a best curve fit 
from Eqn. 1 with $b$ values $\leq 1.5$ defined as a $\mathrm{V}$-shape and $b$ values $>1.5$ as $\mathrm{U}$ shaped. For example, Figure 11 shows a $b$ value of 1.78 demonstrating a U-shaped cross-sectional profile.

The form ratio $\left(F_{r}\right)$ was used by Hirano and Aniya (1988) in order to better understand where on the spectrum between $\mathrm{V}$ and $\mathrm{U}$-shape the cross section of glacial valleys fell. The form ratio is equal to $D / W$, with $D$ defined as the valley depth and $W$ as the valley width. Hirano and Aniya (1988) defined two regimes based on their findings; the Rocky Mountain regime where $b$ values rise with increasing $F_{r}$ and the Patagonia and Antarctica regimes where larger $b$ values are associated with decreasing $F_{r}$. They indicated that the form ratio could be used independently of any other valley shape analysis. Several other studies such as (Brook et al., 2004) have followed this example, however, there is disagreement over the proper usage of the form ratio Harbor (1992) suggested that the form ratio should only be used in conjunction with the power law, stating it has no merit on its own due to the fact that two valleys could have the same form ratio but two very different profile shapes. Based on these arguments, the form ratio as proposed by Hirano and Aniya (1988) was not used in this study.

There is some error associated with determining $b$ values. James (1996) compared the usefulness of the power law (Eqn. 1) and the quadratic equation for determining the cross-sectional shape of glacial valleys,

$y=m x^{2}+n x+p$ 
where $y$ is elevation above sea level, $x$ is the lateral distance from the valley's axis; $m, n$, and $p$ are constants with $m$ representing valley form. After examining three valleys from the northwestern Sierra Nevada James (1996) concluded that the value of $b$ can differ depending on operational decisions of where to begin each curve fit analysis. The parameter $b$ can be sensitive to valley bottom morphology and so, in areas where processes such as fluvial action or rock fall debris have modified the valley floor, $b$ can misrepresent the true valley shape. Values of $b$ can also be significantly altered based on the terminating height chosen for the curve fits. For example, a curve fit analysis of a transect in Little Yosemite Valley at a height of $200 \mathrm{~m}$ produces a $b$ value of 1.35 , but a height of $50 \mathrm{~m}$ produces a $b$ value of 1.97 , two very distinct representations of valley cross-sectional shape. It is also essential that all transects be drawn perpendicular to topography as any deviation can again result in a $b$ value that is not truly representative of valley form.

James (1996) concluded that a quadratic equation was less sensitive to valley bottom morphology errors although convexity, asymmetry, and polymorphism in the valley cross-sectional profile made the usage of the quadratic equation inappropriate. Due to the high amount of visual complexity observed within many of the valleys selected for field studies and widespread acceptance, the power law was selected as the most representative equation of valley form for this study. Mitigation of the various issues mentioned above was attempted through the selection of transects dominated by exposed bedrock with little or no signs of other 
processes having operated on the area, limiting the curve fit height to a maximum glacial elevation of $200 \mathrm{~m}$, and taking all transects perpendicular to the valley axis.

Large scale joints $(>0.5 \mathrm{~km})$ measured from aerial photographs were also incorporated into the analysis. Each of the six valleys was divided into $5 \mathrm{~km}^{2}$ areas extending along the length of the valley where measurements had been taken in the field. The strike of glacial flow and all visible joints were measured using the ruler tool in Google Earth ${ }^{\circledR}$ at an eye altitude of $\sim 10,920 \mathrm{~m}$ (Figure 12). The difference in orientation between the large scale joints and valley trend, the delta strike, was also calculated.

\section{Results}

An independent sample t-test was run on the RMS and $b$ values from the six river valleys. The average RMS value for $\mathrm{U}$-shaped glacial valleys $(78.13 \pm 9.19$ $1 \sigma$ ) was not significantly different $(\alpha=0.05)$ than the average RMS value for Vshaped glacial valleys $(75.25 \pm 9.401 \sigma)$ (independent samples t-test: $\mathrm{n}=42, \mathrm{p}=$ 0.476). There was no apparent correlation between $b$ and RMS (Figure 13), suggesting that valley profiles are not dependent on rock mass strength.

Despite this lack of correlation, there are several points worth noting. Each valley, with the exception of Deadman Canyon, has cross-sectional profiles that range from strongly $\mathrm{V}$-shaped to distinctly $\mathrm{U}$-shaped. Although the $b$ values from Deadman Canyon imply that it is predominantly U-shaped, it too has a spectrum of profiles from barely U- shaped to highly U-shaped. Interestingly, Deadman 
Canyon was the only valley to have a slight negative linear relationship between RMS and $b$ (Figure 13). In addition, all the valleys except Deadman Canyon have at least one profile with two halves of the transect with different $b$ values. For example, Tenaya Canyon has a profile with a $b$ value of 1.9 for the left side and 1.2 for the right side.

Because it has been suggested that the distance between joints plays a role in valley profile shape (Duhnforth, et al. 2010), a plot of joint spacing vs. $b$ was created to determine if such a relationship could be identified from the current study's field data (Figure 14). Joint spacing values extended from $0.3-20 \mathrm{~m}$ with the majority of spacing falling between $0.4-2.5 \mathrm{~m}$. Values for $b$ ranged from $3.4-$ 1.0 with no discernible correlation with joint spacing.

In order to investigate the role of joint orientation, poles to the plane for the strike and dip of each of the smaller scale joints were plotted on an equal area lower- hemisphere stereonet. All of the valleys have a significant grouping of shallowly dipping joints with clusters of vertical to sub-vertically dipping joints (Figure 15), indicating the presence of two distinct perpendicular joint sets which can form fractured rock blocks of sufficient size for quarrying (Figure 16).

A plot of $b$ vs. the mode of the delta strike (Figure 17) suggests a clustering of joint direction between $25^{\circ}$ and $45^{\circ}$ indicating that the majority of the jointing is obliquely oriented to the valley trend and glacial flow direction. A percent frequency histogram (Figure 18) exhibits a similar tendency in that the delta strike 
values with the two highest percentages are $45^{\circ}$ and $25^{\circ}$. The delta strike with the third highest percentage is $5^{\circ}$ which appears to deviate from figure seventeen's results.

\section{Discussion}

Former glacial valleys that have retained their presumed original fluvial shape in Scotland and Iceland were documented by Brook et al. (2004). Their study focused on how bedrock properties in five morphologically and lithologically distinct terrains influenced valley morphology away from continent-continent collision zones. The power law, form ratio, and quadratic equation were used as measures of glacial valley cross- profile shape that were then compared to rock mass strength. Their results showed that while valleys with lower rock mass strength values have a distinct $\mathrm{U}$-shape and valleys with higher rock mass strength values tend to be deeper with a considerably less pronounced U-shape profile, the rock mass strength properties that are most important in valley morphology are joint width, spacing, and orientation.

Augustinus (1992) also conducted a similar study in the Southern Alps of New Zealand using a slightly modified version of Selby's (1980) RMS classification and intact strength based on Schmidt hammer measurements. Augustinus (1992) used the form ratio, the shape factor, glaciated valley joint density, and the coefficients $a, b$ and $c$ from the quadratic formula to quantify valley morphology in granite, gneiss, greywacke, and schist. A lack of relationship was found between the rock 
intact strength properties and morphometric variables such as the form ratio, valley cross-sectional shape, and the shape factor, but a positive relationship was shown when plotting valley profile against rock mass strength, indicating steeper slopes and narrower valleys in regions with higher rock mass strength.

Error Analysis

A comparison of average rock mass strength (RMS) and valley shape ( $b$ from Eqn. 1) from Brook et al.'s (2004) data and the current study was done to corroborate the experimental techniques of the present study. Brook et al.'s (2004) average RMS value of 78.75 and average $b$ value of 1.48 in granite agree reasonably well with the current study's average RMS of 78.91 and average $b$ value of 1.42 , confirming that my experimental techniques of the present study are acceptable. A comparison of $b$ values could not be made with Augustinus' (1992) data as he did not use the power law, but his average RMS value of 82.5 is not significantly greater than 78.7 or 78.9.

Despite demonstrating a lack of correlation between rock mass strength and valley shape, this study shows that there are both $\mathrm{V}$ and $\mathrm{U}$-shaped profiles throughout formerly glaciated valleys within the central and southern Sierra Nevada. This brings forth the question of how these two morphologies can exist simultaneously in an area of such extensive glaciation. The idea that rivers are capable of carving U-shaped valleys and that the cross-sectional profiles within the Sierra Nevada range were set by pre-glacial fluvial processes was suggested by Schaffer (1997). This concept is illustrated with Zion Canyon in Zion National 
Park, Utah. It has a broad, flat floor that gradually merges with walls whose heights rival that of Yosemite Valley to form a wide U-shaped valley (Grater, 1947). Zion Canyon consists predominantly of the Navajo sandstone which can be as resistant to abrasion as granite (Sklar and Dietrich, 2001). There are two factors that undermine the strength of this particular type of sandstone. The first is a weaker cement, making it less resistant to the sapping processes which are known to form broad, flat-floored U-shaped profiles (Kochel and Piper, 1986; Gulick and Baker, 1990). The second is the presence of closely spaced joints and joints of various lengths, which lowers the rock's resistance to erosion even further (Bahat et al., 1995). These two factors, combined with extensive biannual flooding events, have led to the U-shaped profile of Zion Canyon, an area that has never been previously glaciated (Grater, 1947). If Schaffer's (1997) interpretation is correct, similar processes to the ones operating in Zion Canyon can be applied to the Sierra Nevada valleys during their formation.

That the Sierra Nevada mountain range was formed during the Cretaceous is not a new concept (e.g. Wahrhaftig, 1965). A recent review of the numerous studies supporting this concept (Gabet, 2014) indicates that there is sufficient evidence to justify an origination of the Sierra Nevada during the Cretaceous. Instead of the current warm temperate climate (Kottek et al., 2006) with seasonal rains, western California was wet and tropical during the early Cretaceous through the midEocene (Minnich, 2007). A warmer and wetter climate leads to increased chemical 
weathering of the felsic minerals within granitic rocks, leaving the more resistant minerals such as quartz and feldspar to a loose material called gruss (White, 1945; Hall et al., 1989). The presence of joints increases the surface area available for weathering which, coupled with a tropical environment, increases granite erosion rates (White, 1945). This weakening of a normally resistant rock may have led to increased fluvial erosion within the Sierra Nevada during the Cretaceous/Paleogene and created morphologies similar to that of Zion Canyon today.

Two separate processes in an open system leading to the same result are known as equifinality (Grezov and Drazin, 1997). Equifinality is an idea that has been used extensively in psychology, biology, medicine, and a variety of other fields. Recently, it has been applied to the field of geological sciences including aspects such as the modeling of hydrologic and geomorphic systems (e.g., Beven, 1996; Phillips, 1997; Beven, 2006), paleoenvironmental reconstruction of alluvial fans in New Zealand (Nicholas and Quine, 2010), and comparison of erosional processes in the formation of shore platforms (Cruslock et al., 2010). Campbell and Twidale (1995) reviewed distinct landforms associated with granite and their development through specific mechanisms and demonstrated that multiple processes are able to produce the same topographic feature. This idea of equifinality can be applied to the formation of Sierra Nevada valleys. In his investigation of Yosemite Valley, Matthes (1930) acknowledged that up to $300 \mathrm{~m}$ of the valley's depth could have 
been due to fluvial erosion, supporting the interpretation that rivers may have contributed a much greater proportion of valley development than originally thought.

\section{Future studies}

The development of joints within the Sierra Nevada presents the most problematic aspect in understanding their role in glacial erosion and valley morphogenesis. The presence of exfoliation jointing, shallowly dipping fractures parallel to the surface on which they form, was noted by Bergbauer and Martel (1999) and Martel (2006) at Olmstead Point in Yosemite National Park. In addition to these shallowly dipping breaks in the rock, vertical and sub-vertical

jointing is also recognized in the Sierra Nevada (Segall, 1984), yet little is known about their formation relative to glaciation and their temporal distribution. Based on an age of $\sim 80$ mya from K/Ar dating of chlorite and epidote K/Ar within fractures in the Sierra Nevada, Segall et al. (1990) put initial joint formation at shortly after the intrusion of the pluton. This implies that some jointing within the Sierra Nevada is pre-glacial, but the rate at which new joints form is unknown. Many microfractures were observed in the field (Figure 20); presumably, these will evolve into joints large enough and deep enough for the next glacier to quarry. If the time-span for the development of these microfractures into extensive jointing is longer than the time between glaciations, the bedrock may not be sufficiently fractured to induce quarrying, thus reducing the effectiveness of glacial erosion. A 
quantification of the timescales over which jointing is sufficient for glaciers to quarry rock is, thus, essential in order to better constrain the rates of glacial erosion.

Studies into fracture mechanics have concluded that the stresses generated during glaciation have the potential to contribute to the acceleration of jointing within the bedrock (Leith, et al., 2011; Hallet 1995; Morland and Boulton, 1975). Under glaciers, high stresses sufficient to form fractures, can occur along the edges of rock steps (Hallet, 1995; Iverson, 1991; Morland and Boulton, 1979). Harbor (1995) has emphasized the importance of these sub-glacial joints as weaknesses that can then be exploited for further erosion by future glacial events. While it is possible that sub-glacial stress induced fracturing may be the principal method through which granite is sufficiently weakened, spatial constraints on joint propagation must also be understood.

Anderson (2014) investigated seasonal variations on the subglacier hydrologic system and progression of surface irregularities in bedrock over thousands of years. A model of glacial erosion supported the importance of subglacial joint formation and block size in the quarrying of rock. If a block is too large, a glacier will be unable to transport it through quarrying and abrasion becomes the primary form of rock removal, reducing the rate of erosion. Field observations from the current study not only noted the occurrence of joint bounded blocks (Figure 16) at varying heights up the valley walls in each of the six sites visited, but several of the valley 
blocks were found with glacial polish on their upper surfaces (Figure 19). Many of the observed blocks also have joint spacing within the 1-3 m range noted by Duhnforth et al. (2010). This observation along with other models of quarrying processes (Iverson, 1991; Cohen et al., 2006; Hooyer, et al., 2012; Iverson, 2012), suggest that there was insufficient fracturing of the blocks during glaciation for effective quarrying.

Although many studies agree that closely spaced joints are the key to effective glacial quarrying, one element that has not yet been closely examined is the vertical propagation of joint growth. In order for the formation of the stepped topography that is needed to promote quarrying (Cohen, 2006; Hooyer, 2012; Iverson, 2012), perpendicular joint sets must either be continuously formed through sub-glacial stresses or be present at depth. Jahns (1945) observed that some exfoliation joints can extend up to $\sim 100 \mathrm{~m}$ at depth. Ziegler et al. (2013), attempted to date exfoliation joints in the Central Alps by mapping their distribution above and below ground and correlating it to various fluvial and glacial erosion patterns within valleys. Their results indicate that exfoliation joints can occur at depths up to $240 \mathrm{~m}$, that they are most established in massive granitic or gneissic rocks away from faulting, and that there were four distinct episodes in which exfoliation joints were generated (lower Pleistocene, middle Pleistocene, upper Pleistocene, and Late Glacial to Holocene).

While it is acknowledged that joint orientation is an important aspect controlling 
rock mass strength (e.g. Augustinus, 1995), very little research has been done to understand its relationship with quarrying. Moore et al. (2009) studied the relationship of rock mass strength properties to rock falls in the north-central Sierra Nevada. They found that although higher rock mass strength did correlate to fewer rock falls, the most important factor in controlling rock fall rate was the orientation of the jointing. Rock wall with joints dipping into the faces were less prone to rock falls than areas with joints dipping out of the rock face. Similarly, recognizing the spatial patterns of jointing and how they affect the mechanism of glacial quarrying is key to being able to fully interpret glacial erosion rates and their ability to alter the landscape. This is particularly important in the Sierra Nevada with its diverse jointing patterns.

Augustinus (1992) and Harbor (1995) have argued that in addition to comparisons such as rock mass strength versus valley cross-sectional profile; the age, mode, and rate of rock slope development should be taken into consideration when evaluating valley morphogenesis. Rock falls have been documented in the granitic cliffs of Yosemite National Park that are associated with exfoliation jointing (Stock et al., 2011). The existence of rock falls throughout this region of the Sierra Nevada is indicative of active erosional processes other than glacial and fluvial which in the area. The extent to which rock falls have contributed to the evolution of valley morphology is a final area of research that should be further explored. 


\section{Conclusion}

Many studies have shown that $\mathrm{V}$-shaped valleys tend to develop in strong rocks and U-shaped valleys are form in weak rock. Data collected for this study matched closely with the average rock mass strength and $b$ values of previous studies but did not find an association between rock mass strength and valley cross-sectional profile.

A better comprehension of subaerial processes such as rock falls and their role in glacial valley morphogenesis is needed before rock mass strength can be linked to valley morphology. A more thorough understanding of the temporal constraints on joint formation in granite and its relationship to glacial cycles will also help to clarify glacial erosion rates in the Sierra Nevada. Rock is an anisotropic material which tends not to conform to idealized models of glacial erosion. The Sierra Nevada is a complex system with an equally complicated geologic history, and the evolution of the valleys within this system may involve considerably more determinants than that of simply abrasion and quarrying. It is likely that the erosional processes which shape valleys are not dominated by a single process but that multiple interactions are occurring at the subglacial interface, and that the idea of equifinality can be applied to the formation of the Sierra Nevada valleys. 

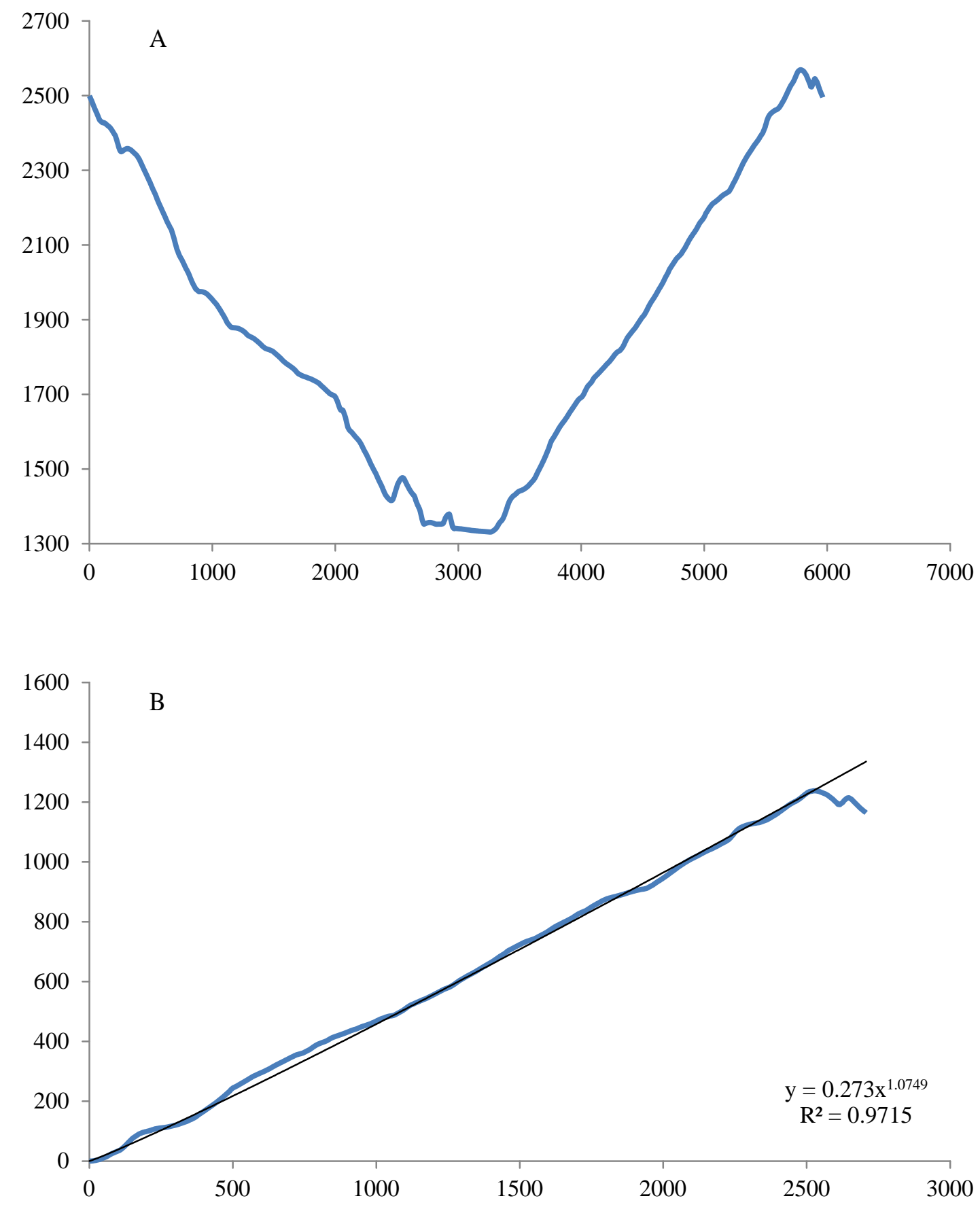

Figure 1. Transect from Tuolumne River valley, Yosemite National Park, California (A). A $b$ value of 1.07 indicates a linear profile or V-shaped valley (B). 


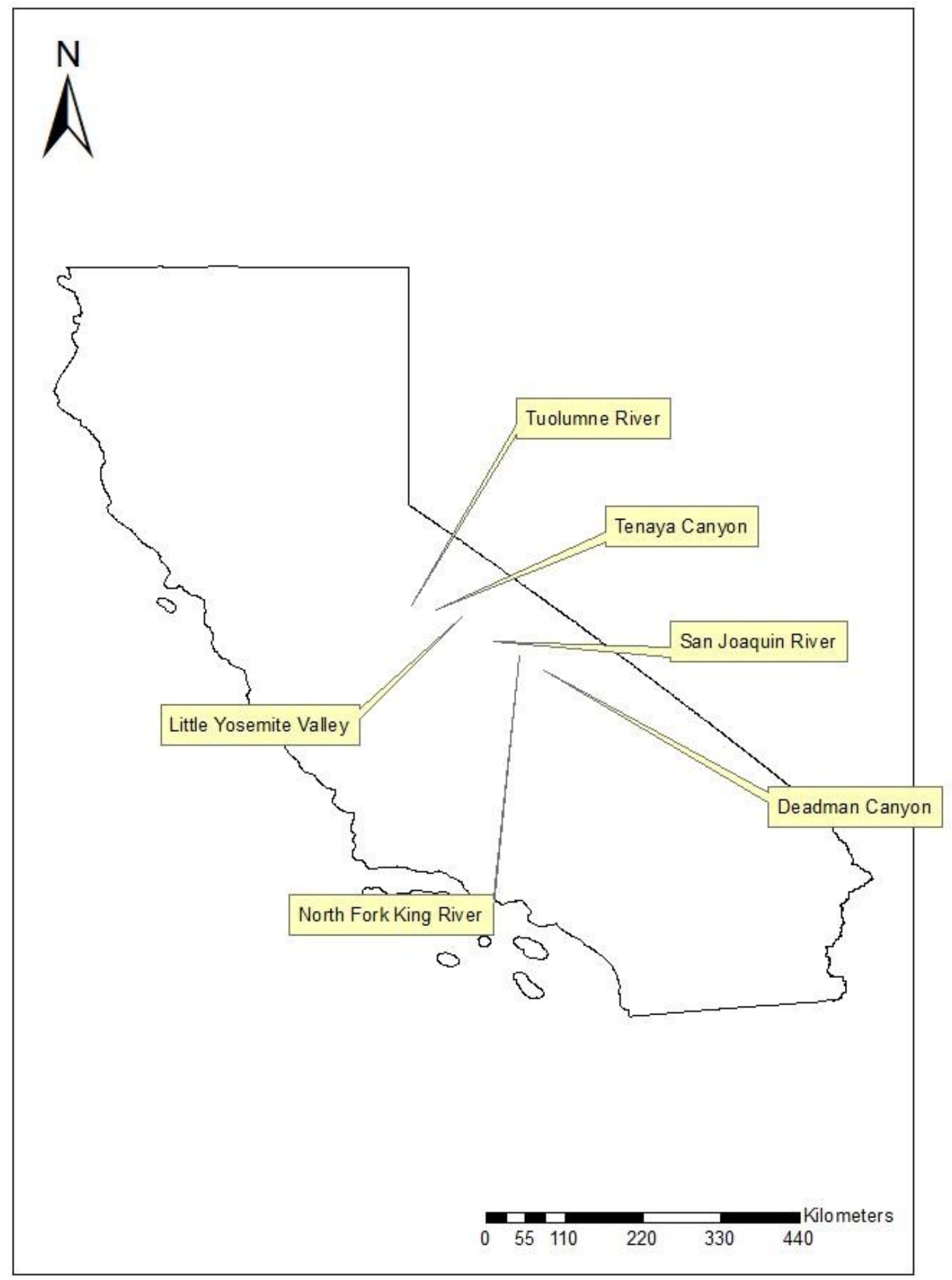

Figure 2. Map of California showing the locations of the six field sites. 


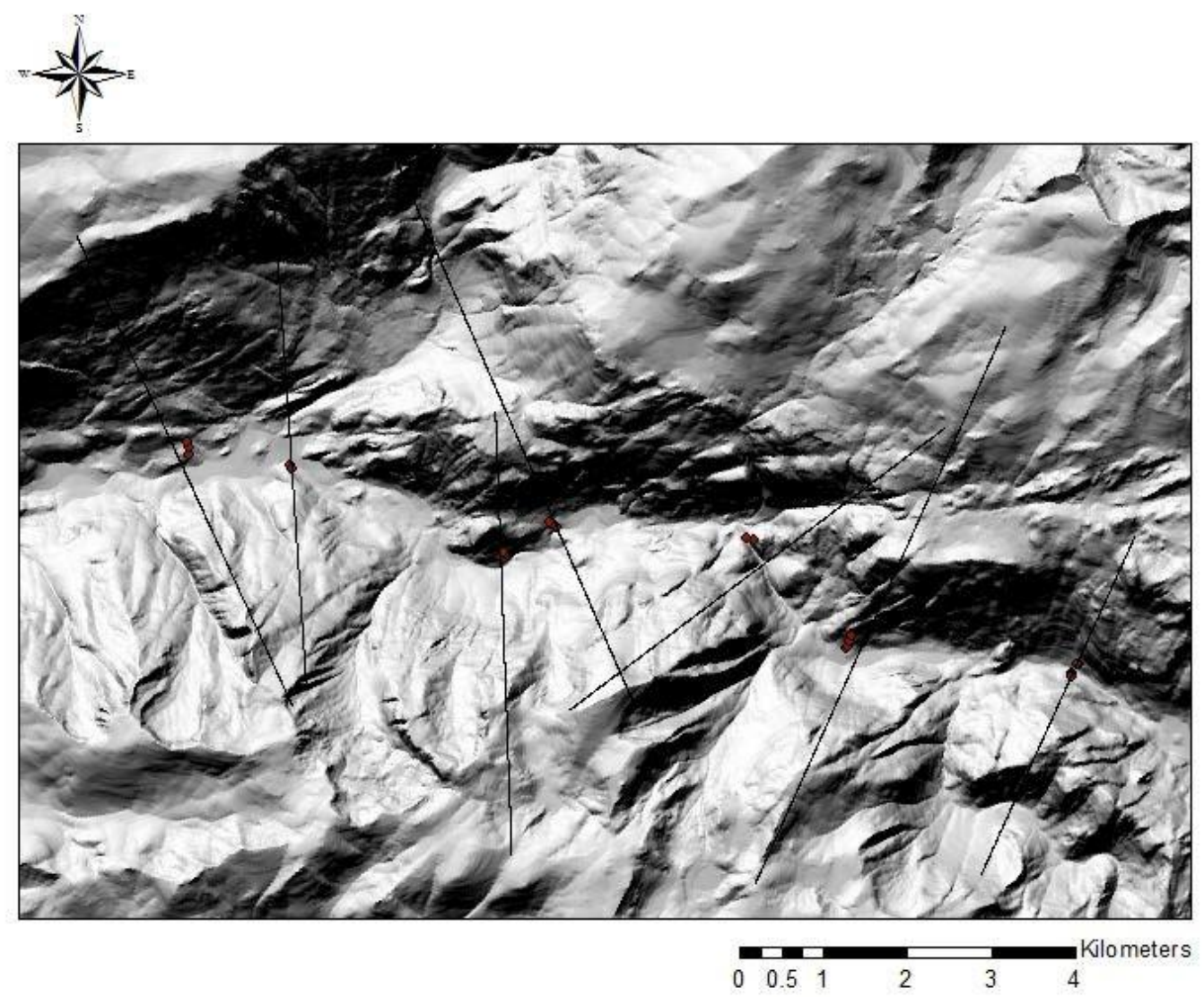

Figure 3. Ten meter DEM of Tuolumne River valley, Yosemite National Park, California. Dots indicate locations of measurements taken along transect (black lines). 


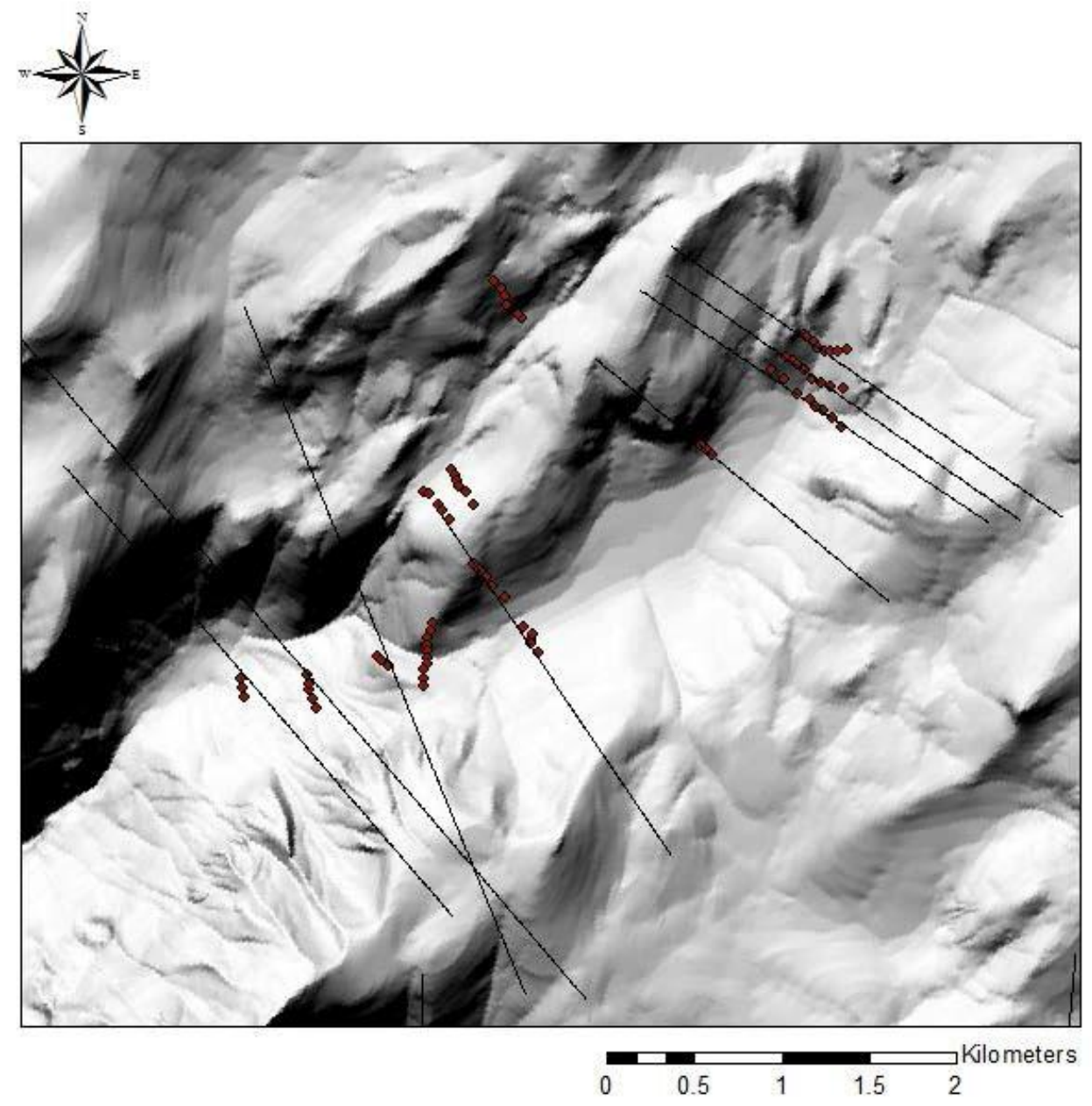

Figure 4. Ten meter DEM of Tenaya Canyon, Yosemite National Park, California. Dots indicate locations of measurements taken along transects (black lines) 


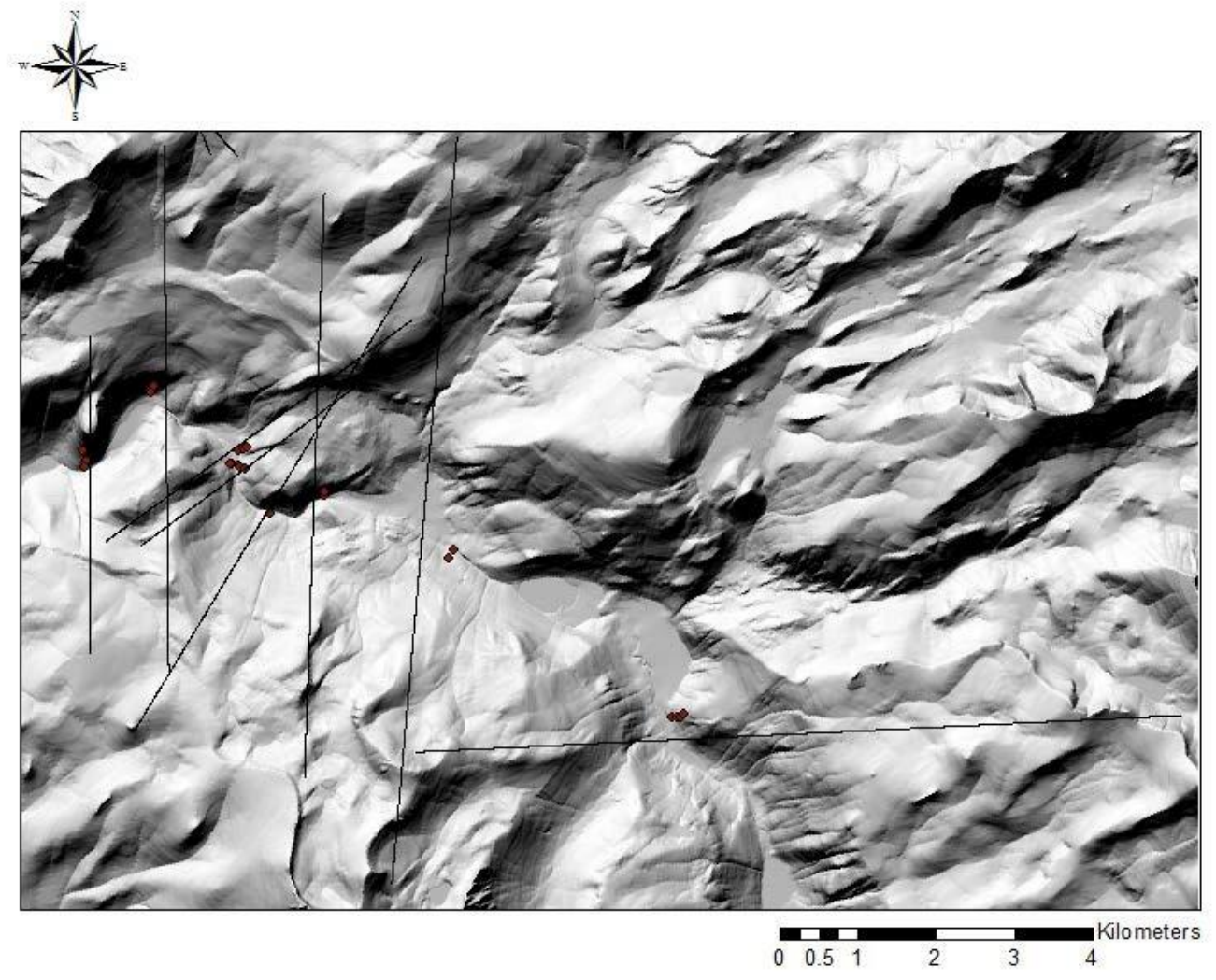

Figure 5. Ten meter DEM of Little Yosemite Valley, Yosemite National Park, California. Dots indicate locations of measurements taken along transect (black lines). 


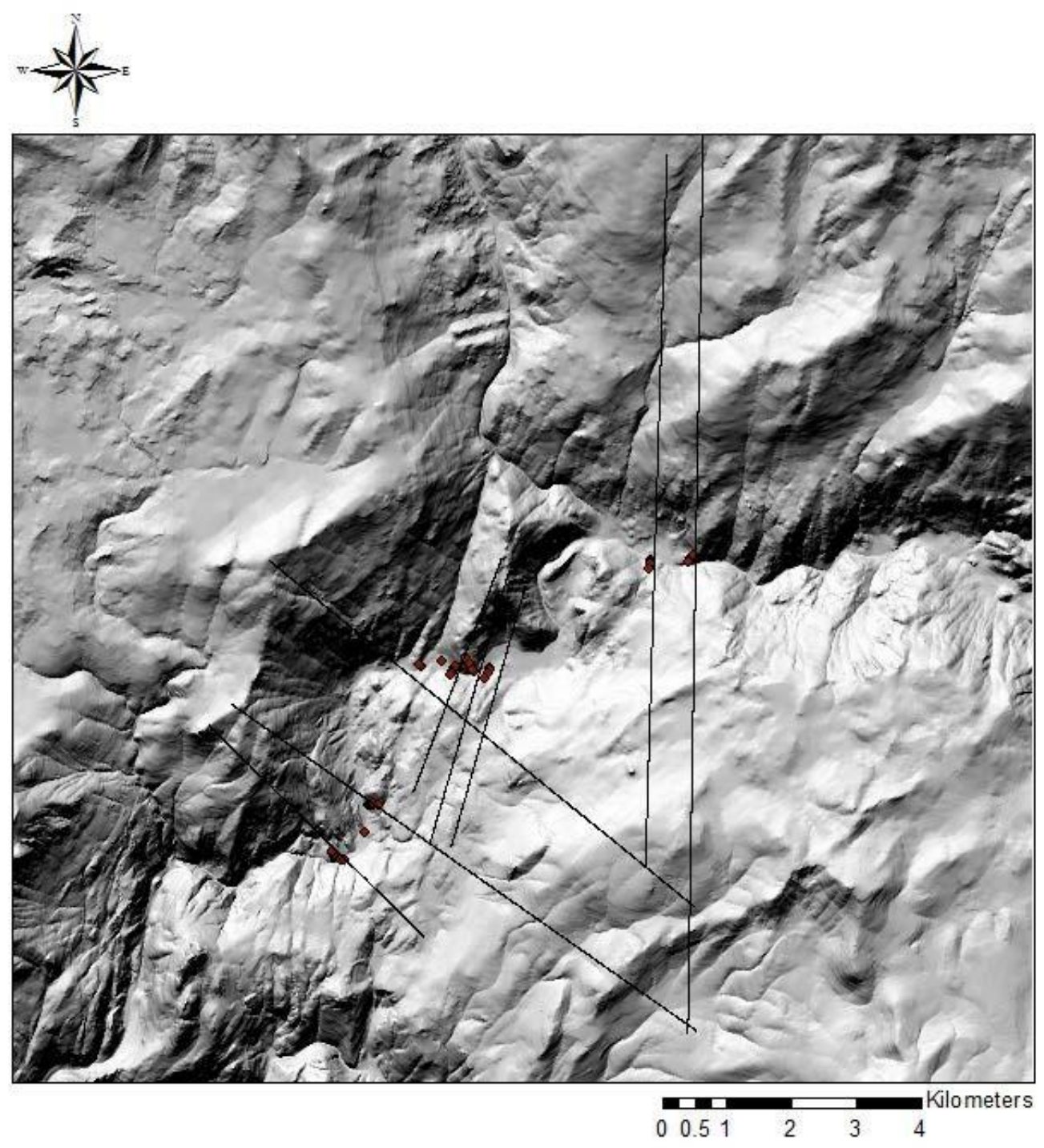

Figure 6. Ten meter DEM of San Joaquin River, Sequoia National Forest, California. Dots indicate locations of measurements taken along transect (black lines). 


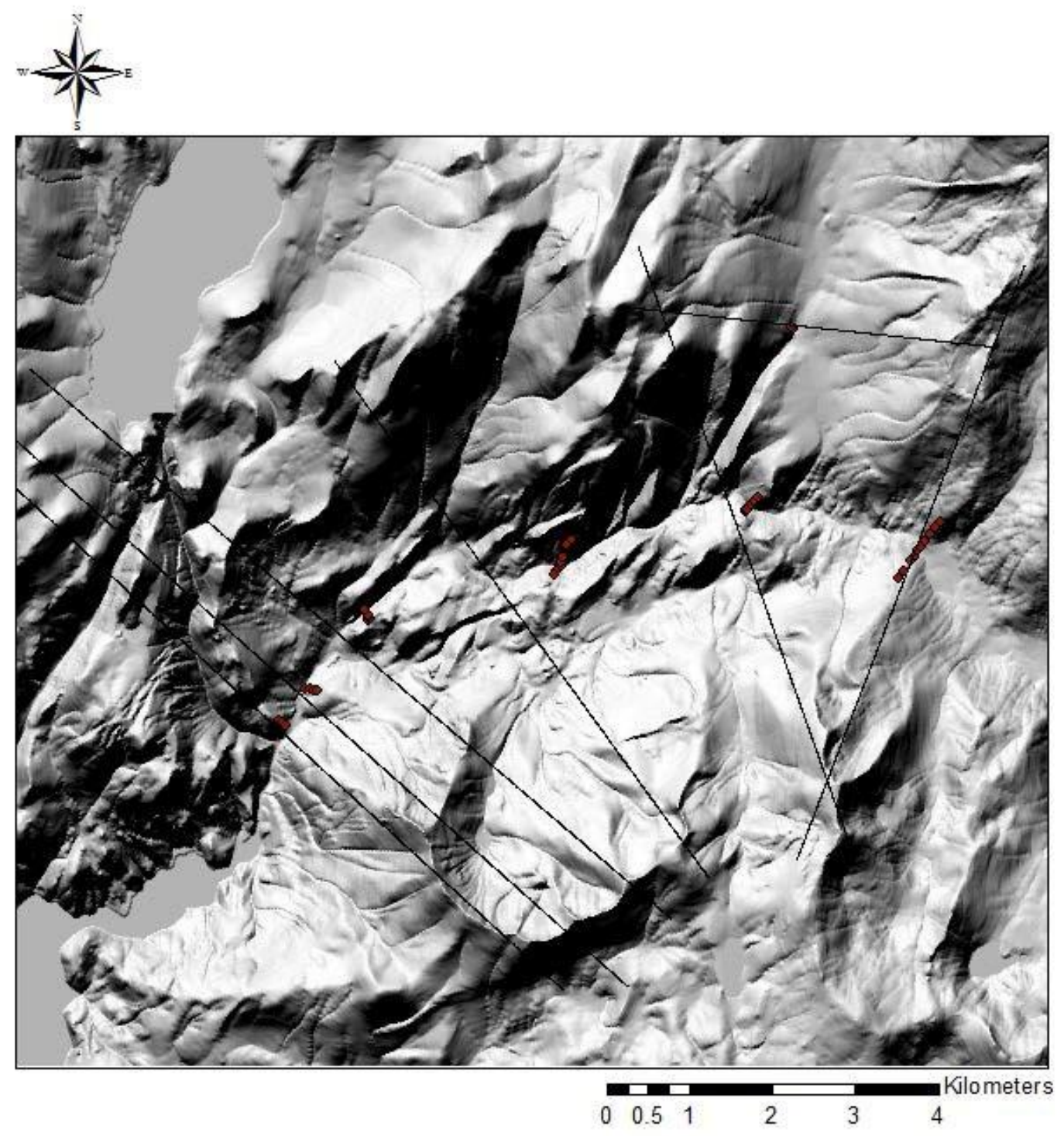

Figure 7. Ten meter DEM of North Fork King River, Sequoia National Forest, California. Dots indicate locations of measurements taken along transect (black lines). 


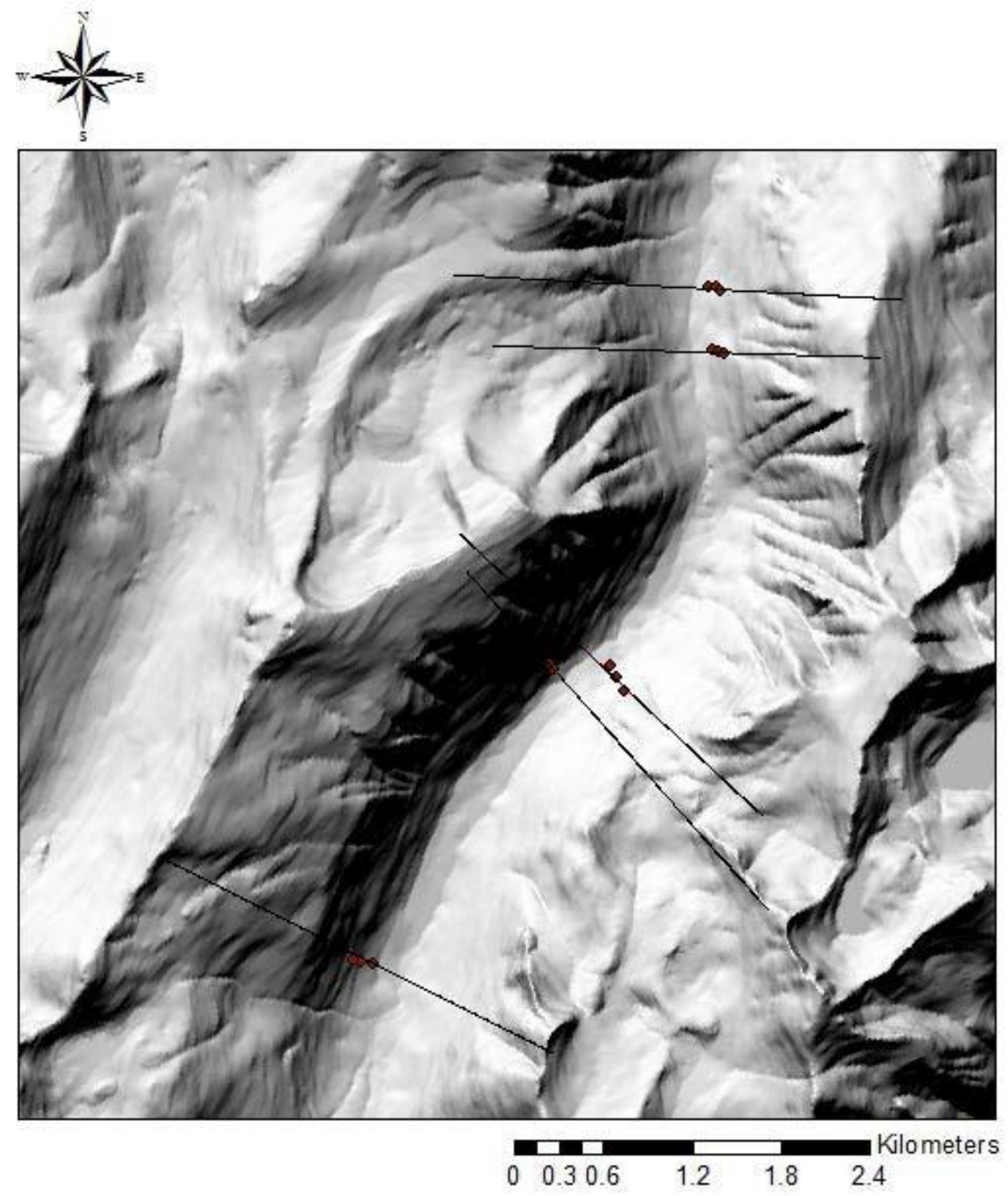

Figure 8. Ten meter DEM of Deadman Canyon, Kings Canyon National Park, California. Dots indicate locations of measurements taken along transect (black lines). 

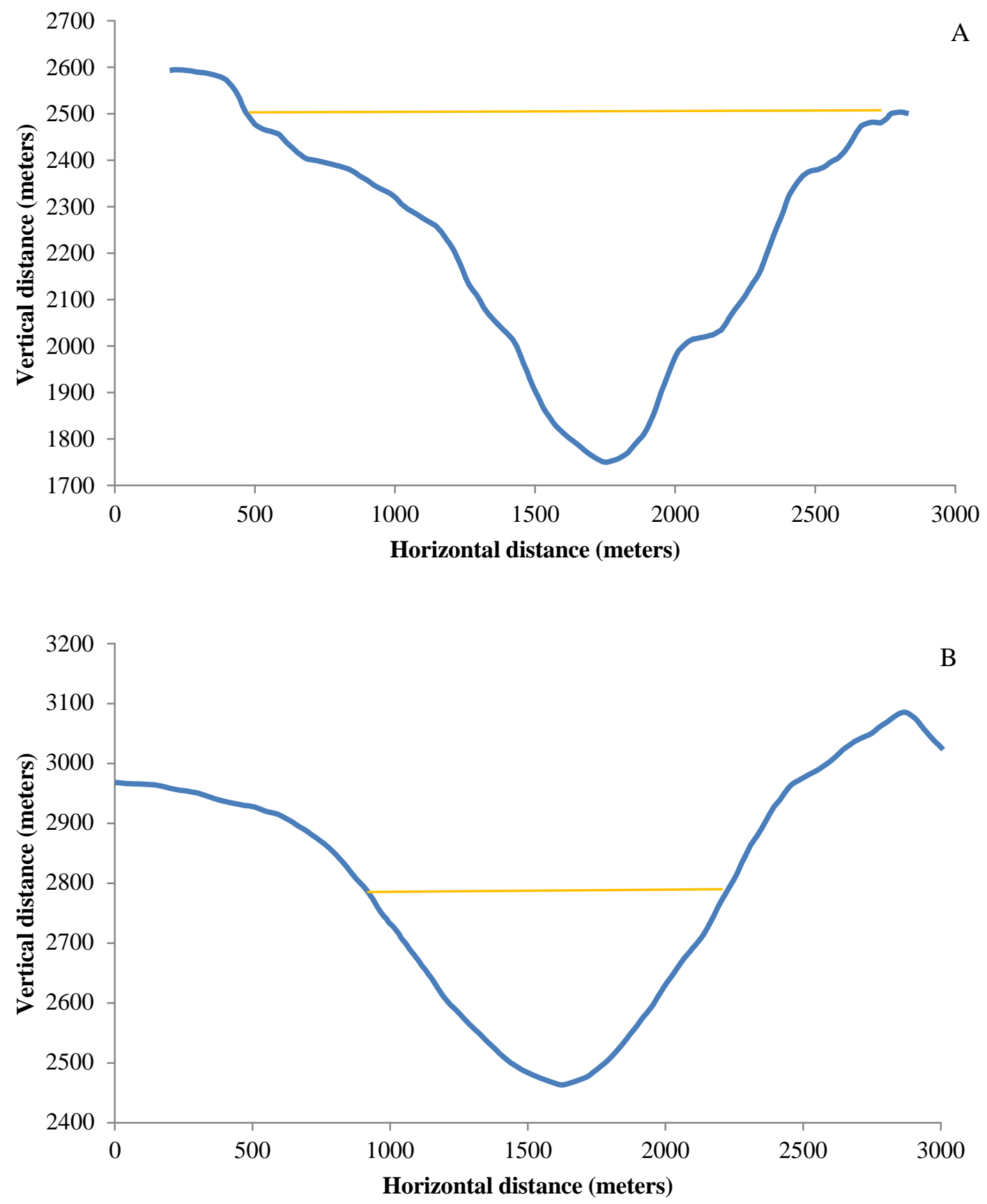

Figure 9. Example of a V-shaped valley (A) and a U-shaped valley from Tuolumne River valley, Yosemite National Park, California (B). The grey lines show the approximate height of the glaciers taken from Matthes (1930) map. 


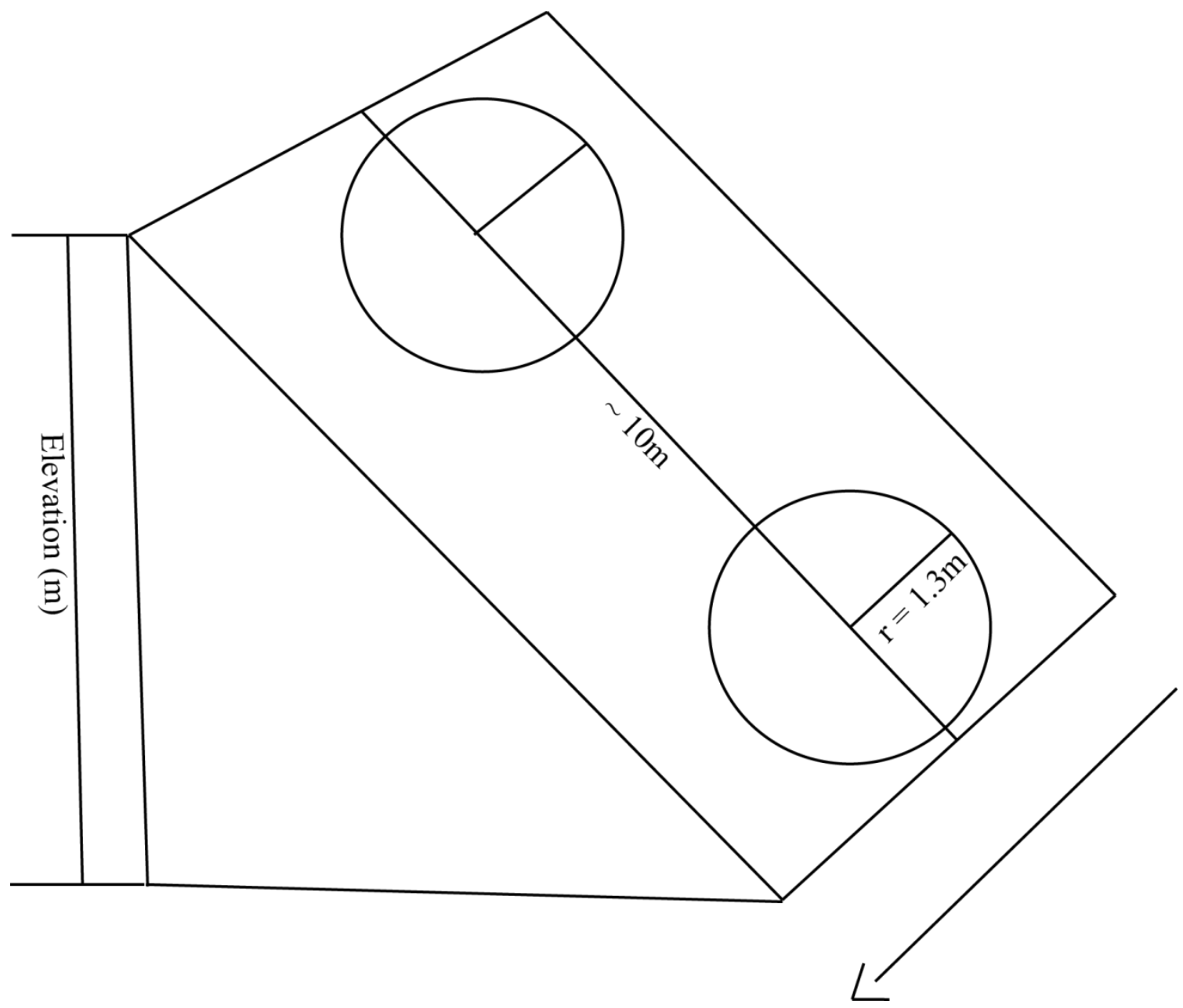

Figure 10. Illustration of one half of a glacial valley with circles indicating an area of $\sim 5 \mathrm{~m}^{2}$ in which all field measurements were taken. Areas are spaced $\sim 10 \mathrm{~m}$ apart. Arrow shows down valley direction. 


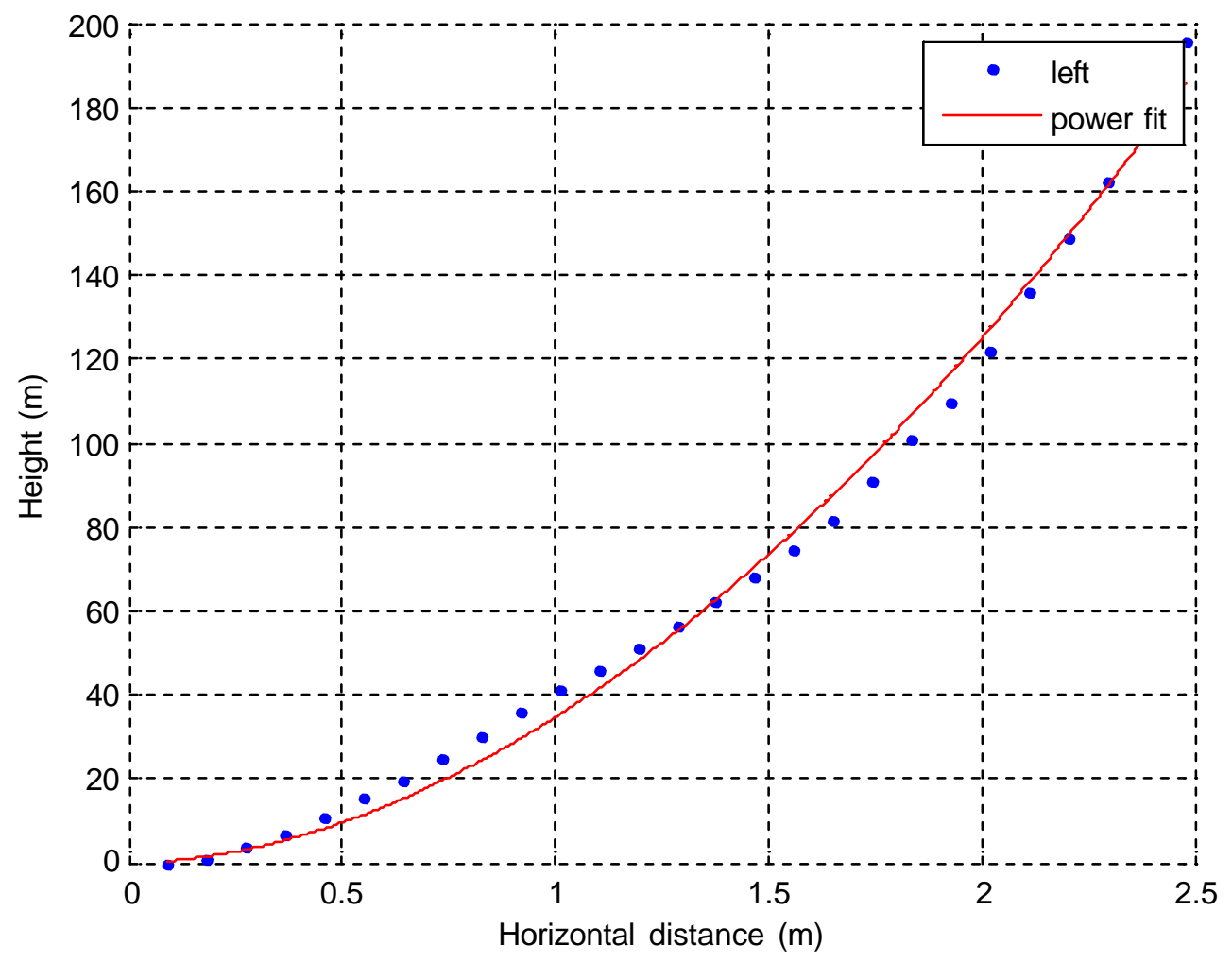

Figure 11. Matlab curve fit for the left half of a transect in Tuolumne River valley with a $b$ value of 1.8 indicating a $\mathrm{U}$-shape. 


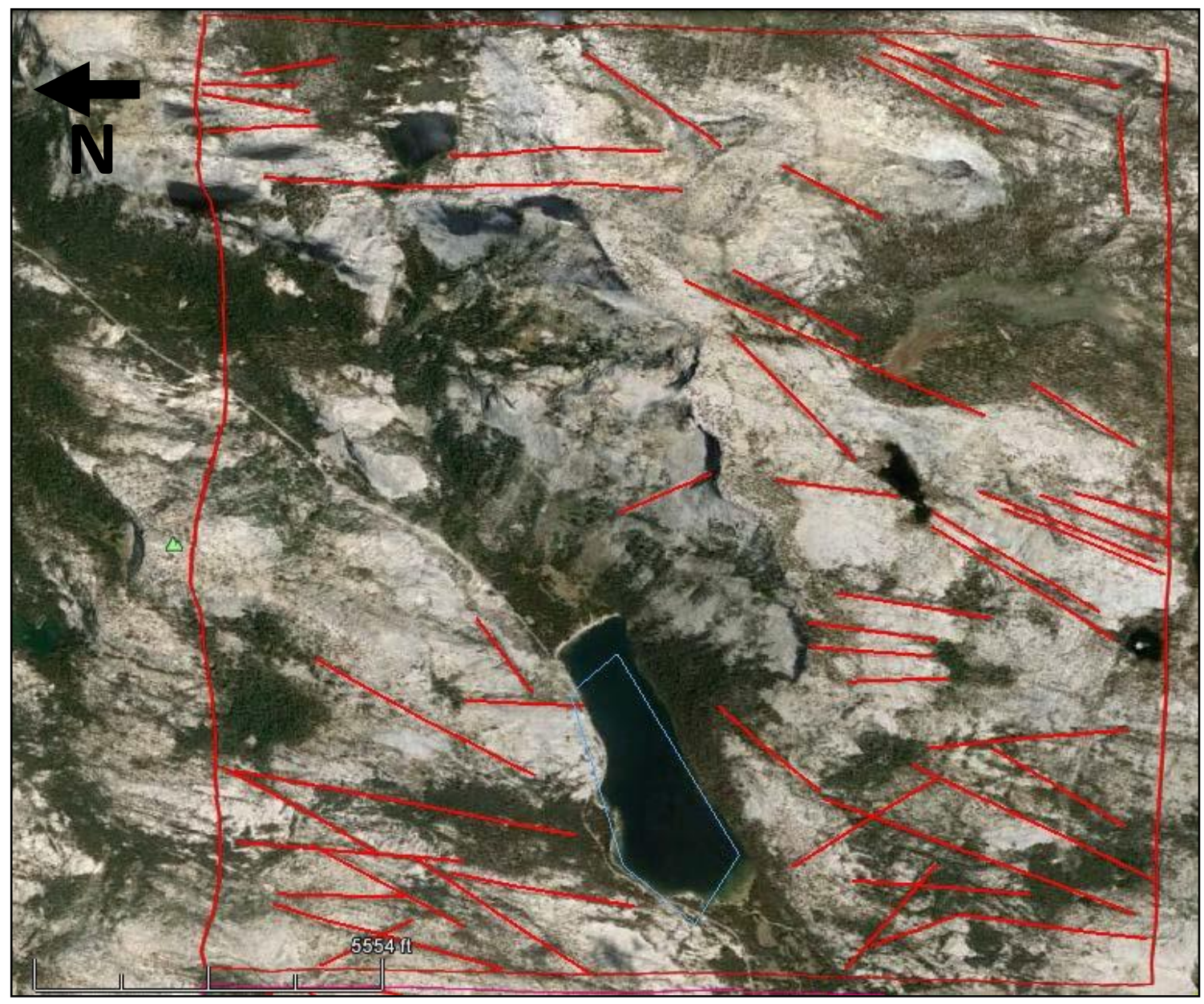

Figure 12. Google Earth image of large scale jointing $(>0.5 \mathrm{~km})$ in a $5 \mathrm{~km}^{2}$ area near Tenaya Lake, Yosemite National Park, California with an eye altitude of $\sim 10,920 \mathrm{~m}$. 

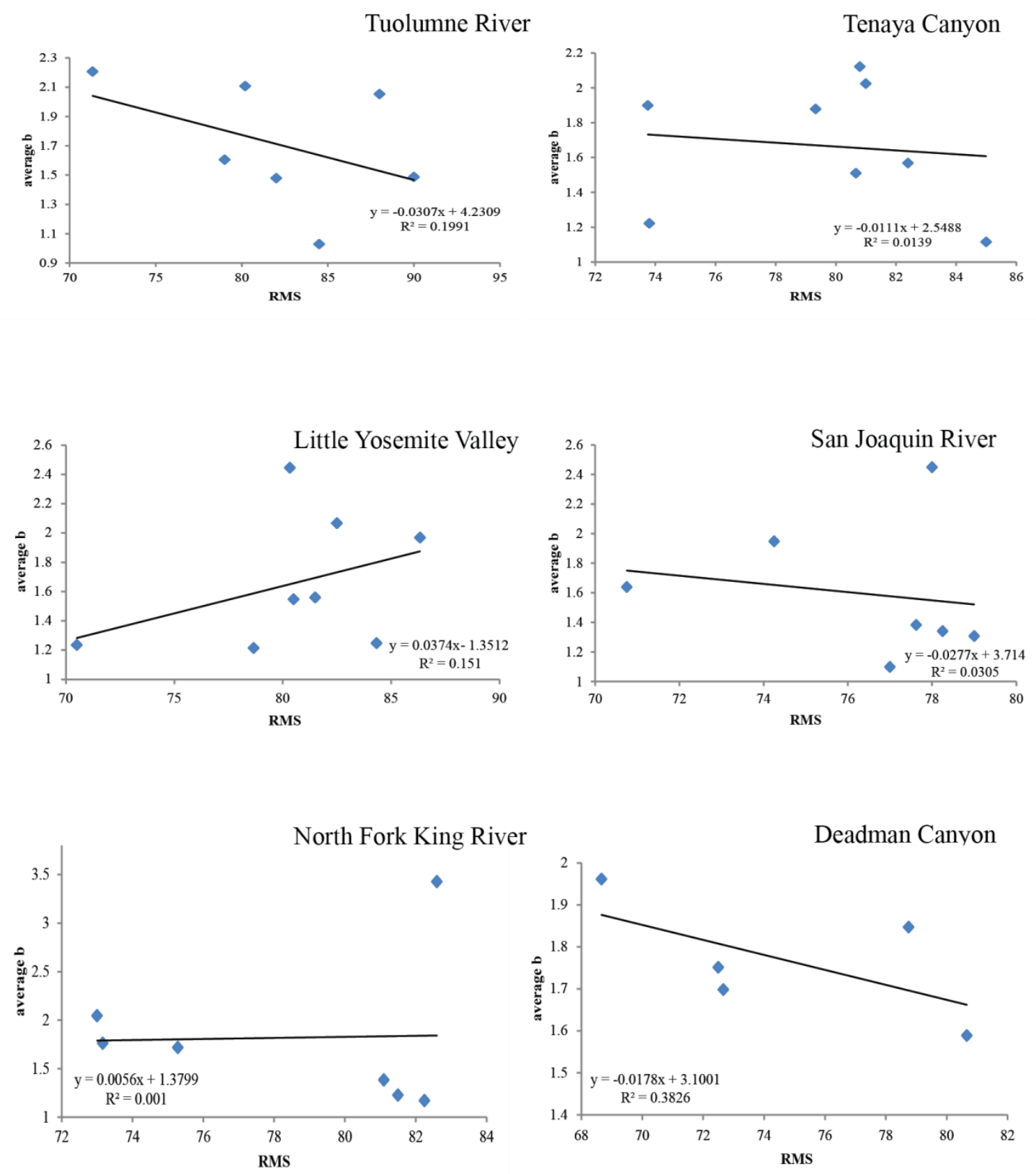

Figure 13. Graphs showing valley shape (b) and rock mass strength (RMS) for each of the six sites surveyed. All six show no correlation between $b$ and RMS with the highest $\mathrm{R}^{2}$ value being 0.38 for Deadman Canyon. 


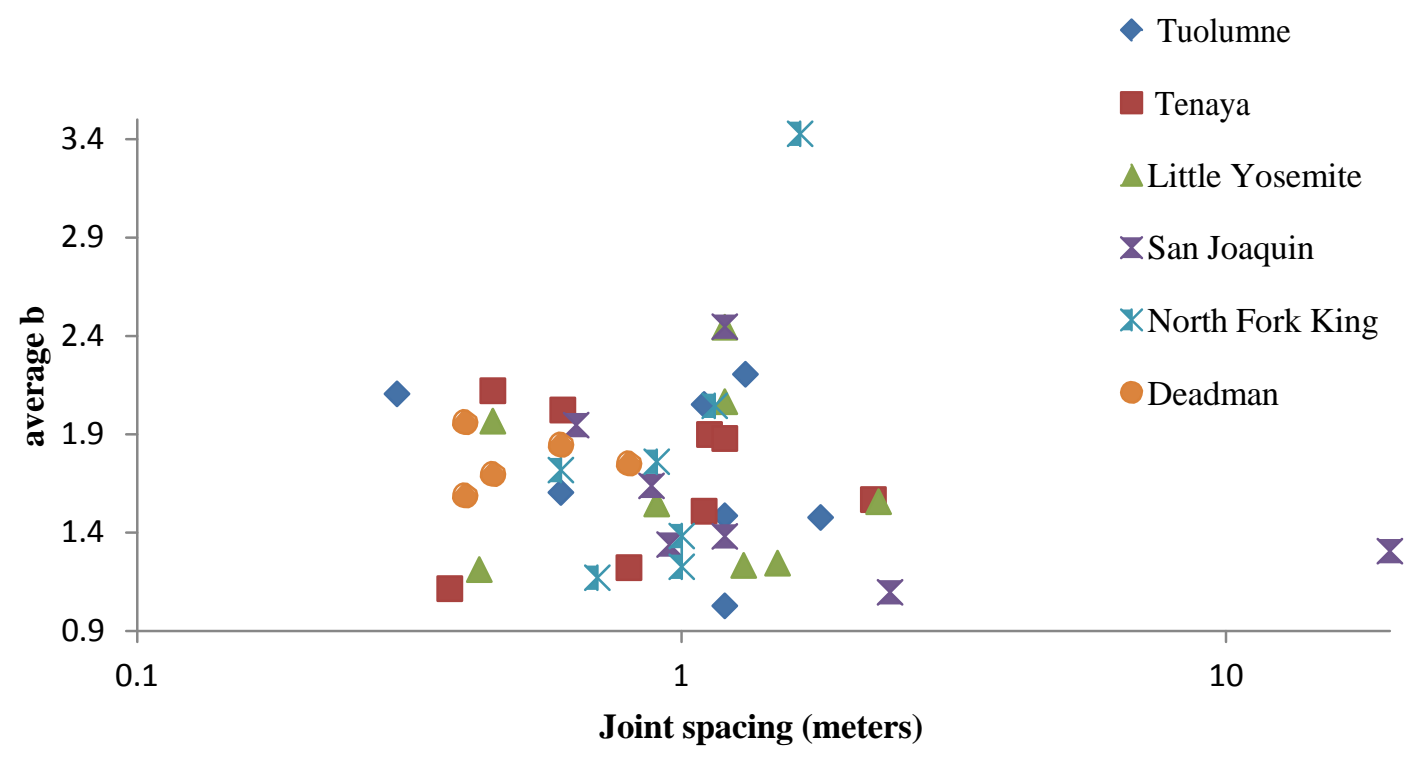

Figure 14. Median joint spacing for the six Sierra Nevada valleys plotted against the average valley cross-sectional shape $(b)$. Although there is no relationship between joint spacing and $b$ the majority of the spacing values fall between $0.3 \mathrm{~m}$ and $2.4 \mathrm{~m}$, roughly the ideal distance for glacial quarrying. Note logarithmic scale on x-axis. 

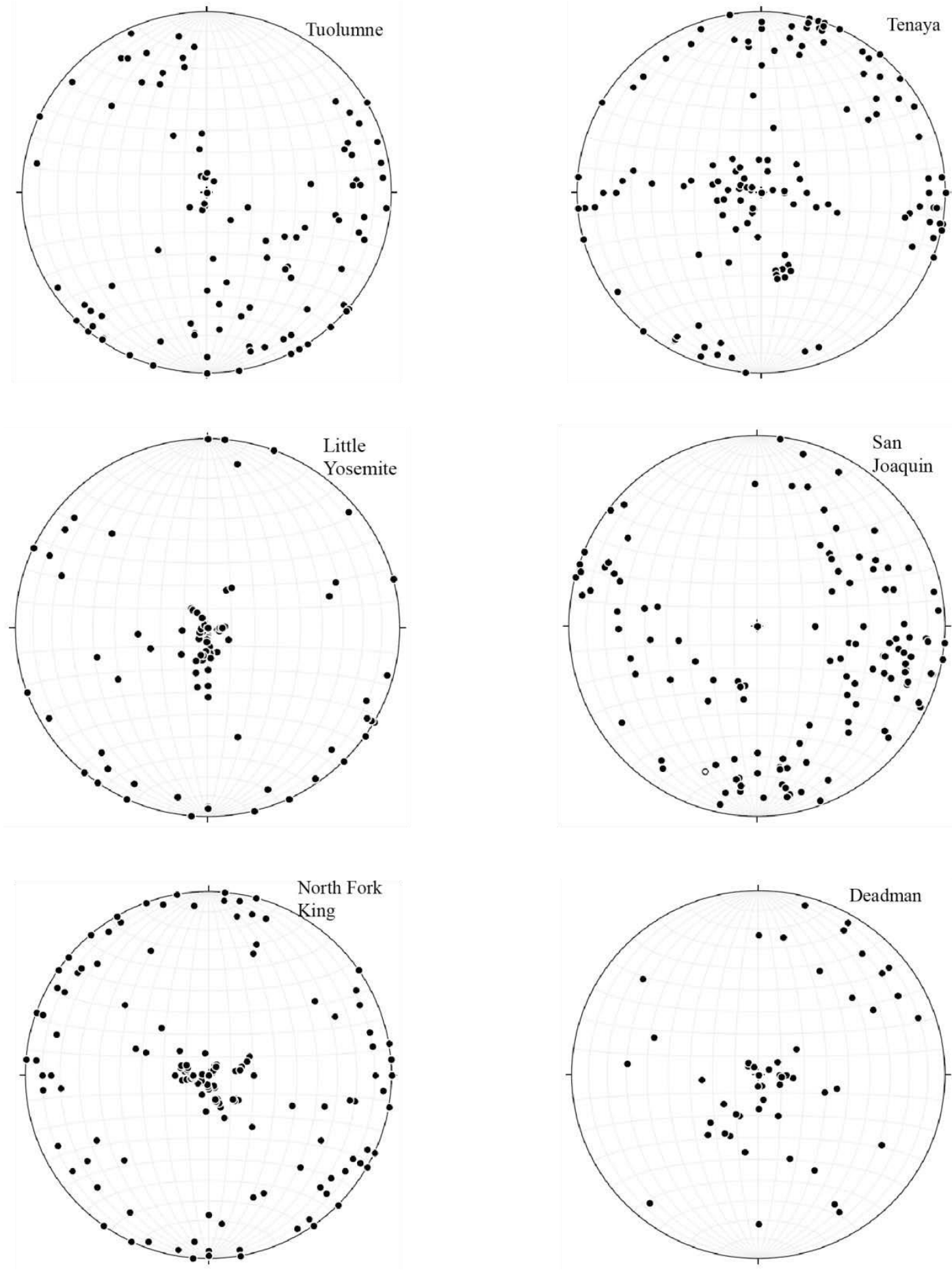

Figure 15. Equal area projection stereonets of the six field sites. All six stereonets show a distribution of shallowly dipping joints as well as sub-vertical to vertically dipping joints. Each valley exhibits a number of perpendicular joints which form fracture bounded blocks, an ideal geometry for glacial quarrying. 


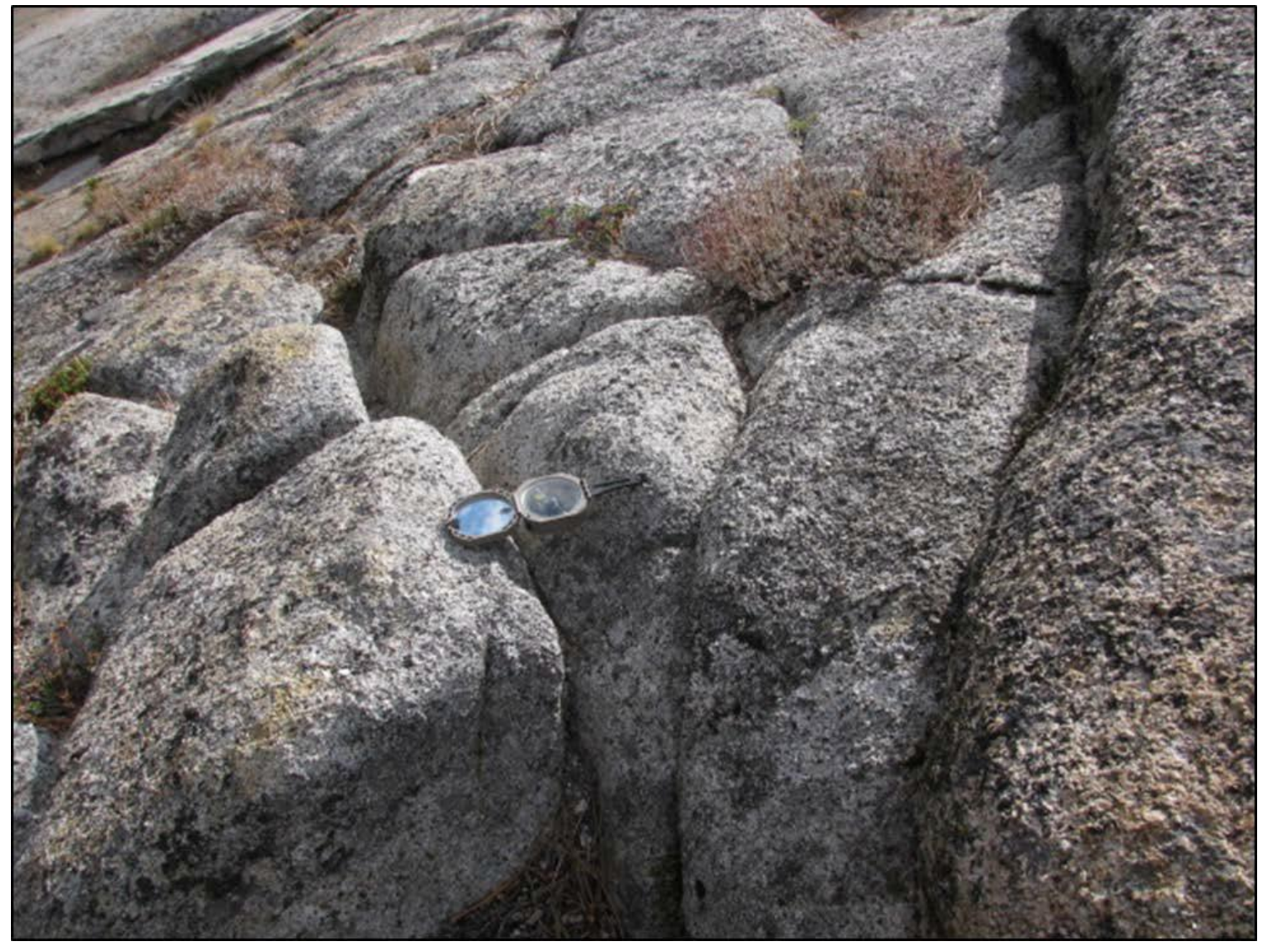

Figure 16. Photo of joint bounded blocks in Tenaya Canyon with $<1 \mathrm{~m}$ joint spacing and stepped topography, an ideal situation for glacial quarrying. Brunton compass for scale. 


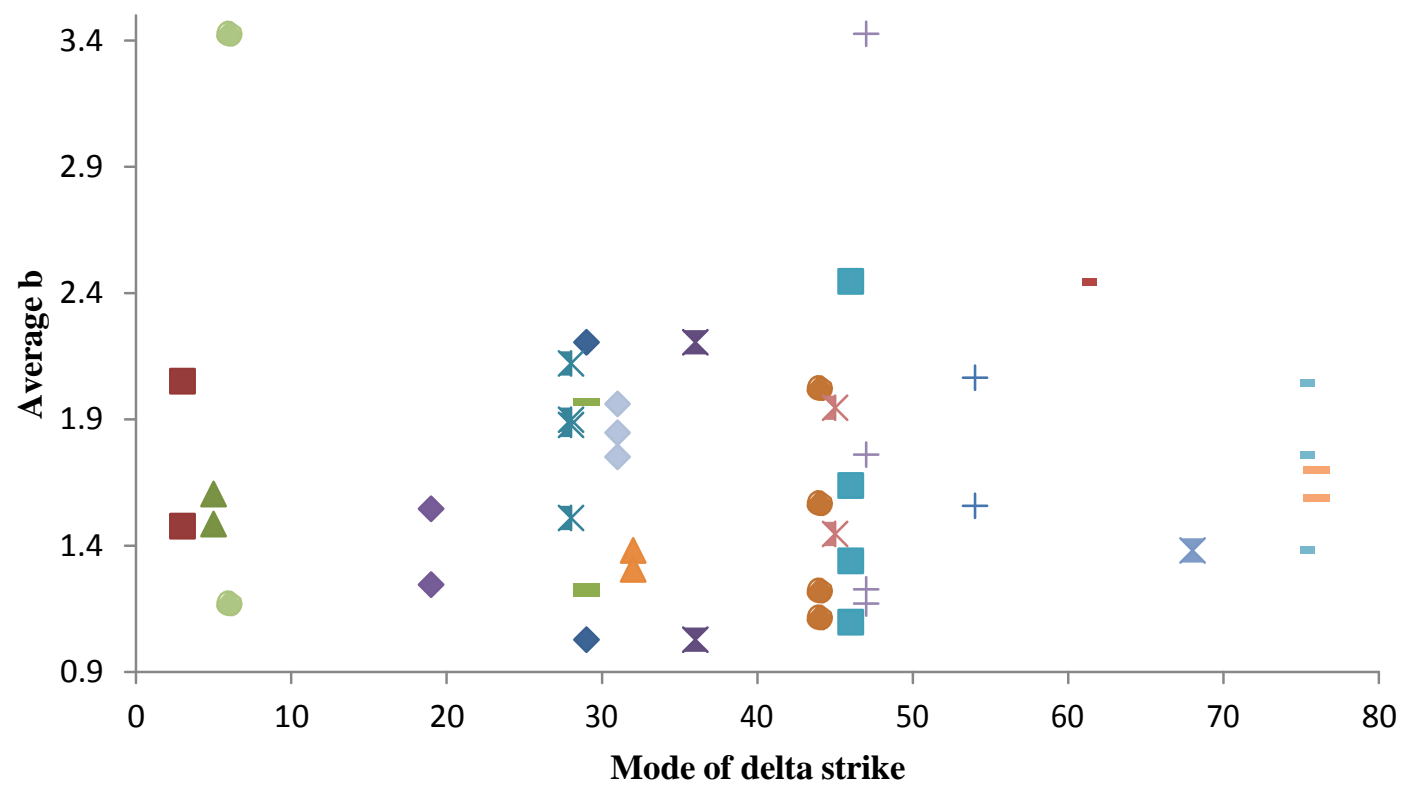

$\checkmark$ Tuolumne $1 \& 2 \quad$ Tuolumne $3 \& 4 \quad \Delta$ Tuolumne $5 \& 6$

又Tuolumne $7 \& 8 \quad$ XTenaya $1 \& 2 \quad$ Tenaya $3 \& 4$

+Little Yosemite $1 \& 2$-Little Yosemite $3 \& 4$-Little Yosemite 5 \& 6

$\checkmark$ Little Yosemite $7 \& 8 \quad$ San Joaquin $1 \& 2 \quad \triangle$ San Joaquin $3 \& 4$

XSan Joaquin $5 \& 6 \quad$ \&San Joaquin $7 \& 8 \quad$ North Fork King $1 \& 2$

+ North Fork King $3 \& 4$-North Fork King $5 \& 6$-Deadman $1 \& 2$

$\diamond$ Deadman $3 \& 4$

Figure 17. Mode of the delta strike vs. average $b$ for 19 reaches from the six valleys surveyed. The clustering of the joints implies that the majority of the large scale joint orientation is $20^{\circ}-45^{\circ}$, obliquely oriented to the longitudinal axis of the valley, although no correlation between delta strike and $b$ values is evident. 


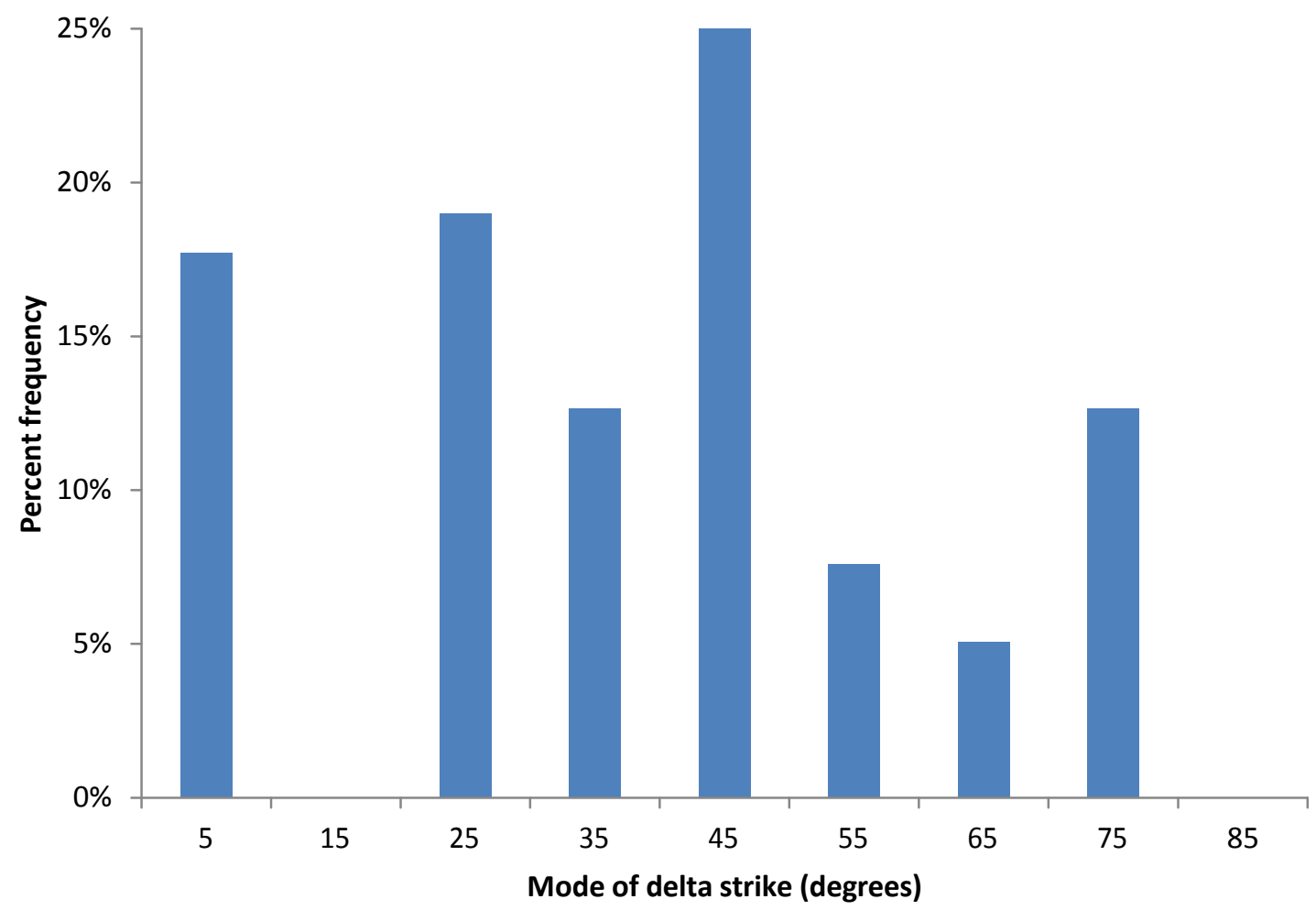

Figure 18. Percent frequency histogram of the mode of delta strike orientations. Note the lack of jointing at $5^{\circ}-25^{\circ}$ and above $75^{\circ}$. 


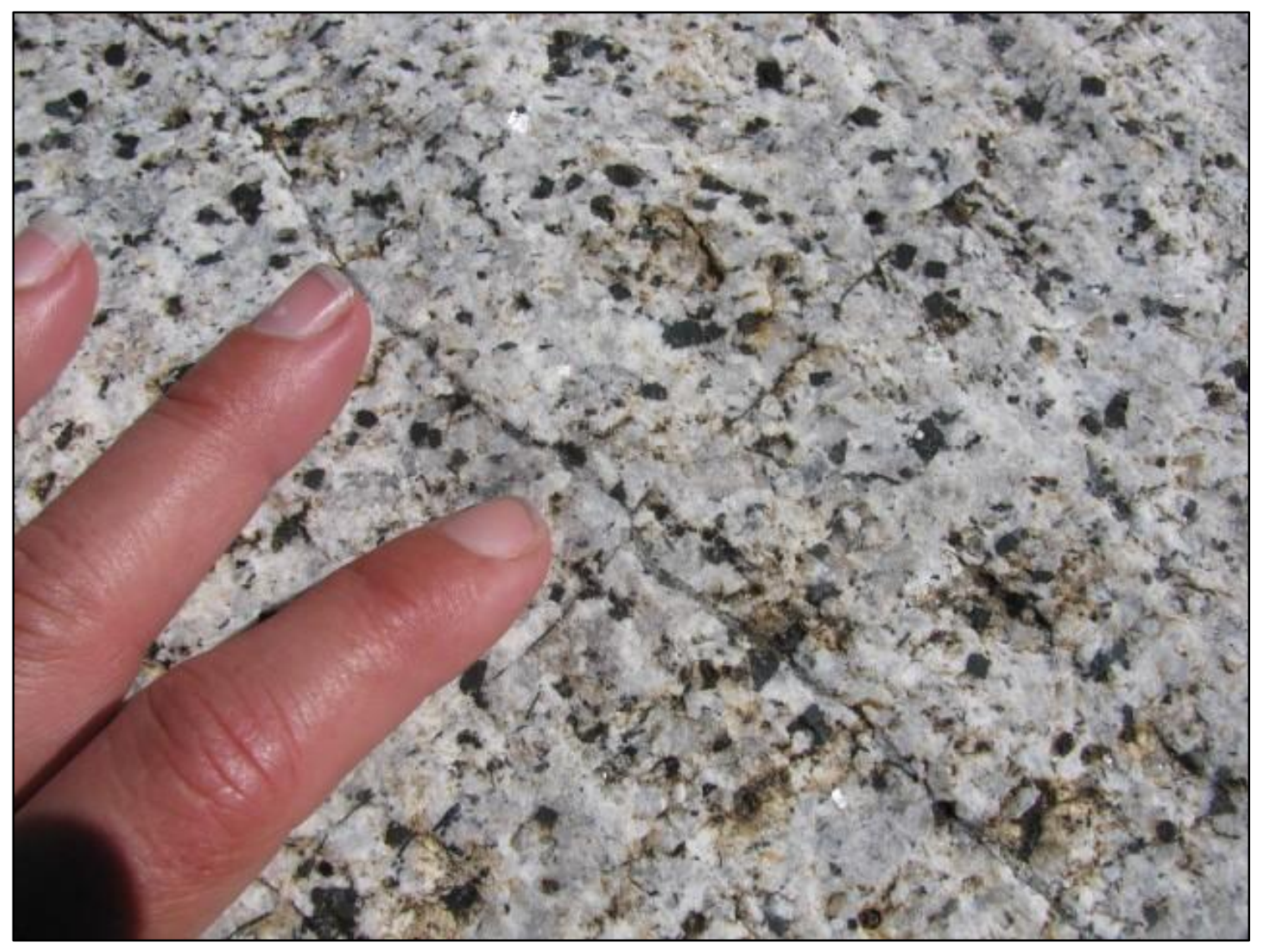

Figure 19. Photo of perpendicular microfractures from Tenaya Canyon. 


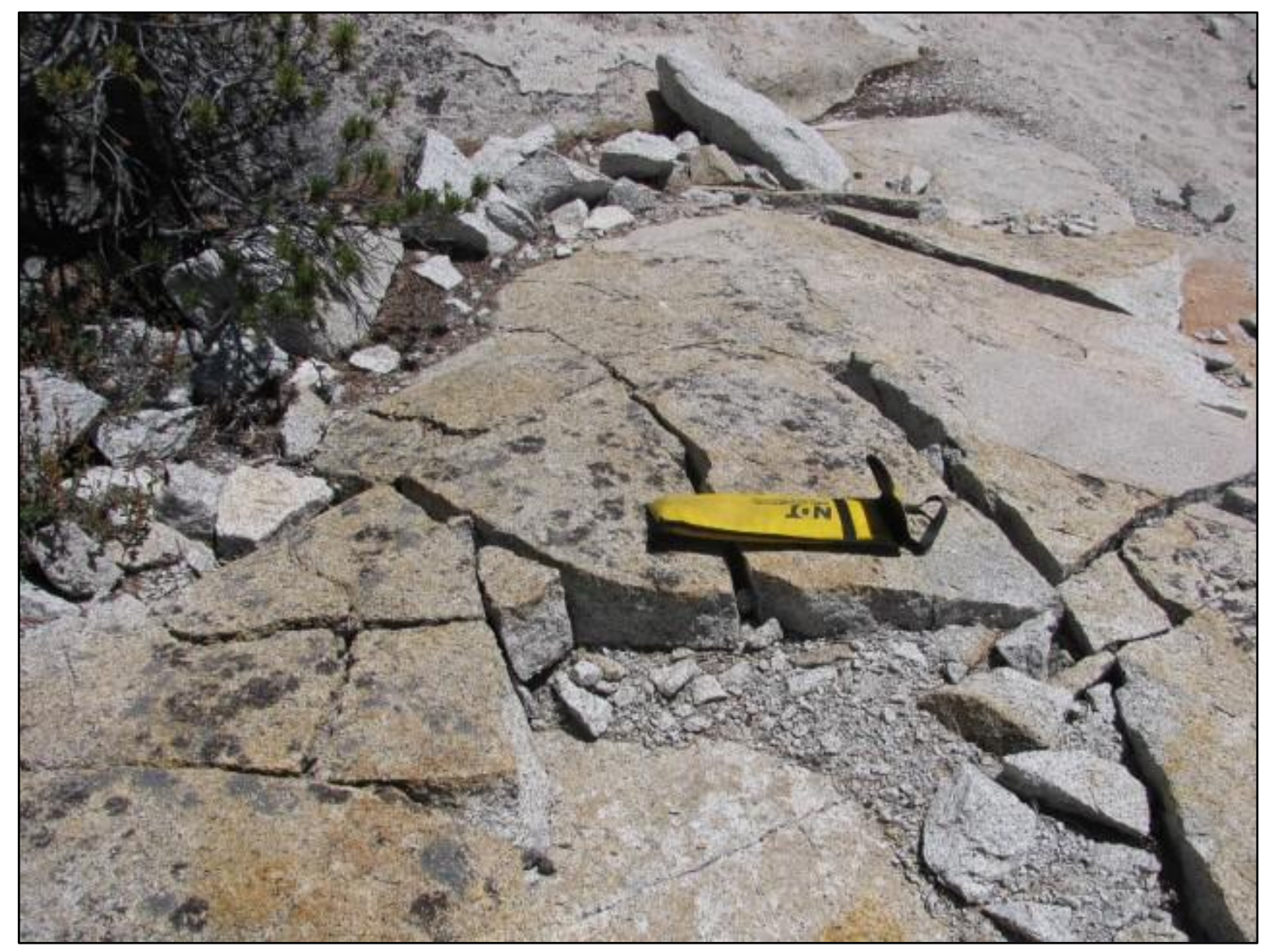

Figure 20. Photo illustrating in situ blocks with joint spacing $<1 \mathrm{~m}$ with glacial polish on their surfaces indicating they were either not moved during glaciation or joints formed post-glacial. 
Table 1. Previous glaciations from the last $\sim 3$ million years within the Sierra Nevada mountains, California (Gillespie and Zehfuss, 2004.

\begin{tabular}{|l|l|}
\hline Glaciation & Mean age (ka) \\
\hline Recess Peak & $14.2-13.1$ \\
\hline Tioga (retreat) & $15-14$ \\
\hline Tioga (start) & $21-20$ \\
\hline Tenaya & 31 \\
\hline Tahoe & $50-42$ \\
\hline Sherwin & c. 820 \\
\hline McGee & $2,700-1,500$ \\
\hline
\end{tabular}


Table 2. Rock mass strength (RMS) classification chart from Selby (1980).

\begin{tabular}{|c|c|c|c|c|c|}
\hline & 1 & 2 & 3 & 4 & 5 \\
\hline Parameter & Very strong & Strong & Moderate & Weak & Very weak \\
\hline $\begin{array}{l}\text { Intact rock } \\
\text { strength (N- } \\
\text { type Schmidt } \\
\text { Hammer 'R') }\end{array}$ & $100-60$ & $59-50$ & $49-40$ & $39-35$ & $34-10$ \\
\hline Weathering & $\mathrm{R}: 10$ & $\begin{array}{l}\text { Slightly } \\
\text { weathered } \\
\text { R:9 }\end{array}$ & $\begin{array}{l}\text { Moderately } \\
\text { weathered } \\
\text { R:7 }\end{array}$ & $\begin{array}{l}\text { Highly } \\
\text { weathered } \\
\text { R:5 }\end{array}$ & $\begin{array}{l}\text { Completely } \\
\text { weathered } \\
\text { R:3 }\end{array}$ \\
\hline $\begin{array}{l}\text { Spacing of } \\
\text { joints }\end{array}$ & $\begin{array}{l}>3 \mathrm{~m} \\
\mathrm{R}: 30\end{array}$ & $\begin{array}{l}3-1 \mathrm{~m} \\
\mathrm{R}: 28\end{array}$ & $\begin{array}{l}0.99-0.3 \mathrm{~m} \\
\mathrm{R}: 21\end{array}$ & $\begin{array}{l}299-50 \mathrm{~mm} \\
\mathrm{R}: 15\end{array}$ & $\begin{array}{l}<50 \mathrm{~mm} \\
\mathrm{R}: 8\end{array}$ \\
\hline $\begin{array}{l}\text { Joint } \\
\text { orientations }\end{array}$ & $\begin{array}{l}\text { Very } \\
\text { favorable. } \\
\text { Steep dips } \\
\text { into slope, } \\
\text { cross joints } \\
\text { interlock. } \\
\text { R:20 }\end{array}$ & $\begin{array}{l}\text { Favorable. } \\
\text { Moderate } \\
\text { dips into } \\
\text { slope. } \\
\text { R:18 }\end{array}$ & $\begin{array}{l}\text { Fair. } \\
\text { Horizontal } \\
\text { dips, or } \\
\text { nearly } \\
\text { vertical (hard } \\
\text { rocks only) } \\
\text { R:14 }\end{array}$ & $\begin{array}{l}\text { Unfavorable. } \\
\text { Moderate dips } \\
\text { out of slope. } \\
\text { R:9 }\end{array}$ & $\begin{array}{l}\text { Very } \\
\text { unfavorable. } \\
\text { Steep dips out } \\
\text { of slope. } \\
\text { R:5 }\end{array}$ \\
\hline $\begin{array}{l}\text { Width of } \\
\text { joints }\end{array}$ & $\begin{array}{l}<0.1 \mathrm{~mm} \\
\mathrm{R}: 7\end{array}$ & $\begin{array}{l}0.1-1 \mathrm{~mm} \\
\mathrm{R}: 6\end{array}$ & $\begin{array}{l}0.99-5 \mathrm{~mm} \\
\mathrm{R}: 5\end{array}$ & $\begin{array}{l}4.9-20 \mathrm{~mm} \\
\mathrm{R}: 4\end{array}$ & $\begin{array}{l}>20 \mathrm{~mm} \\
\mathrm{R}: 2\end{array}$ \\
\hline $\begin{array}{l}\text { Continuity of } \\
\text { joints }\end{array}$ & $\begin{array}{l}\text { None } \\
\text { continuous } \\
\text { R:7 }\end{array}$ & $\begin{array}{l}\text { Few } \\
\text { continuous } \\
\text { R:6 }\end{array}$ & $\begin{array}{l}\text { Continuous, } \\
\text { no infill } \\
\text { R:5 }\end{array}$ & $\begin{array}{l}\text { Continuous, } \\
\text { thin infill } \\
\text { R:4 }\end{array}$ & $\begin{array}{l}\text { Continuous, } \\
\text { thick infill } \\
\text { R:1 }\end{array}$ \\
\hline $\begin{array}{l}\text { Outflow of } \\
\text { groundwater }\end{array}$ & None & Trace & $\begin{array}{l}\text { Slight } \\
<251 / \mathrm{min} / 10 \\
\mathrm{~m} 2 \\
\mathrm{R}: 4\end{array}$ & $\begin{array}{l}\text { Moderate } \\
25-125 \\
1 / \mathrm{min} / 10 \mathrm{~m} 2 \\
\mathrm{R}: 3\end{array}$ & $\begin{array}{l}\text { Great } \\
>125 \\
1 / \mathrm{min} / 10 \mathrm{~m} 2 \\
\mathrm{R}: 1\end{array}$ \\
\hline Total rating & $100-91$ & $90-71$ & $70-51$ & $50-26$ & $<26$ \\
\hline
\end{tabular}




\section{References}

Allmendinger, R., 2014, Stereonet 9, http://www.geo.cornell.edu/geology/faculty/RWA/programs/stereonet.html.

Alpha, Tau R., Wahrhaftig, Clyde, and Huber, N.K., 1987, Oblique map showing maximum extent of 20,000-year-old (Tioga) glaciers, Yosemite National Park, Central Sierra Nevada, California, scale varies with direction, 1 sheet.

Augustinus, P. C. 1995, Glacial valley cross-profile development: the influence of in situ rock stress and rock mass strength, with examples from the Southern Alps, New Zealand: Geomorphology, v. 14, p. 87-97.

Augustinus, P. C., 1992, The influence of rock mass strength on glacial valley crossprofile morphometry: A case study from the Southern Alps, New Zealand: Earth Surface Processes and Landforms, v. 17, p. 39-51.

Bahat, D., Grossenbaker, K., and Karasaki K. 1995, Investigation of Exfoliation Joints in Navajo Sandstone at the Zion National Park and in Granite at the Yosemite National Park by Tectonofractographic Techniques: Lawrence Berkeley Laboratory, University of California, Earth Sciences Division, p. 1-70.

Baker, V.R. 2001, Water and the Martian landscape: Nature, v. 412, p. 228-236.

Bateman, P.C. 1992, Plutonism in the Central Part of the Sierra Nevada Batholith, California: U.S. Geological Survey Paper 1483, p. 1-194.

Bateman, P.C., Kistler, R.W., Peck, D.L., and Busacca, A. 1983, Geologic map of the Tuolumne Meadows quadrangle, Yosemite National Park, California: U.S. Geological Survey, Geologic Quadrangle Map GQ-1570, scale 1:62,000, 1 sheet. 
Bateman, P.C., Lockwood, J.P., and Lydon, P.A. 1971, Geologic map of the Kaiser Peak quadrangle, central Sierra Nevada, California: U.S. Geological Survey, Geologic Quadrangle Map GQ-894, scale 1:62,500, 1 sheet.

Bateman, P.C. and Wahrhaftig, C. 1966, Geology of the Sierra Nevada: Geology of northern California: California Division of Mines and Geology Bulletin, 190, p. 107-172.

Bateman, P.C., 1965, Geologic map of the Blackcap Mountain quadrangle, Fresno County, California: U.S. Geological Survey, Geologic Quadrangle Map GQ-428, scale 1:62,500, 1 sheet.

Bergbauer, S. and Martel, S.J. 1999, Formation of joints in cooling plutons: Journal of Structural Geology, v. 21, p. 821-835.

Beven, K., 1996, Equifinality and Uncertainty in Geomorphological Modeling, in eds. Rhoads, B.L. and Thorn, C.E., The Scientific Nature of Geomorphology: Proceedings of the $27^{\text {th }}$ Binghamton Symposium in Geomorphology held 27-29 September 1996, p. 291313.

Beven, K., 2006, A manifesto for the equifinality thesis: Journal of Hydrology, v. 320, p.18-36.

Birkland, P.W. 1964, Pleistocene glaciation of the northern Sierra Nevada, north of Lake Tahoe, California: The Journal of Geology, v. 72, p. 810-825.

Brakenridge, G.R., Hewsom, H.E., and Baker, V.R., 1985, Ancient hot springs on Mars: Origins and paleoenvironmental significance of small Martian valleys: Geology, v. 13, p. 859-862.

Brook, M.S., Kirkbride, M.P., and Brock, B. W. 2004, Rock strength and development of glacial valley morphology in the Scottish Highlands and northwest Iceland: Geografiska Annaler, v. 86, p. 225-234. 
Boulton, G.S., 1979, Process of Glacier Erosion on Different Substrata: Journal of Glaciology, v. 23, p. 15-38.

Cohen, D., Hooyer, T.S., Iverson, N.R., Thomason, J.F., and Jackson, M. 2006, Role of transient water pressure in quarrying: a subglacial experiment using acoustic emissions: Journal of Geophysical Research, v. 111. DOI: 10.1029/2005JF000439.

Cruslock, E.M., Naylor, L.A., Foote, Y.L., and Swantesson, J.O.H., 2010, Geomorphic equifinality: A comparison between shore platforms in Hoga Kusten and Faro, Sweden and the Vale of Glamorgan, South Wales, UK: Geomorphology, v. 114, p. 78-88.

Duhnforth, M., Anderson, R.S., Ward, D. and Stock G.M., 2010, Bedrock fracture control of glacial erosion processes and rates: Geology, v. 38, p. 423-426.

Gabet, E., 2014, Late Cenozoic uplift of the Sierra Nevada, California? A critical analysis of the geomorphic evidence : American Journal of Science, v. 314, p. 1224-1257.

Gillespie, A.R. and Zehfuss, P.H., 2004, Glaciations of the Sierra Nevada, California, USA, in Ehlers, J. and Gibbard, P.L., eds., Quaternary Glaciations - Extent and Chronology, Part II: New York, Elsevier B.V., p. 51-62.

Goudie, A.S. 2006, The Schmidt hammer in geomorphological research. Progress in Physical Geography 30, 6. 703-713. DOI: 10.1177/3039133306071954.

Graf, W.L. 1970, The geomorphology of the glacial valley cross section: Arctic and Alpine Research, v. 2, p. 303-312.

Grater, R.K., 1947, A Geologic and Geographic Sketch of Zion National Park: ZionBryce Museum Bulletin No. 3 National Park Service, Zion Natural History Museum, 18 p. 
Grezov, C. and Drazin, R., 1997, Equifinality: Functional Equivalence in Organizational Design: Academy of Management Review, v. 22, p. 403-428.

Gulick, V.C. and Baker, V.R. 1990, Origin and evolution of valleys on Martian volcanoes: Journal of Geophysical Research: Solid Earth (1978-2012) v. 95, p. 1432514344.

Gumede, H. and Stacey, T.R. 2007, Measurement of typical joint characteristics in South African gold mines and the use of these characteristics in the prediction of rock falls: The Journal of The Southern African Institute of Mining and Metallurgy, v. 107, p. 335-344.

Hall, A.M., Mellnor, A, and Wilson, M.J., 1989, The clay mineralogy and age of deeply weathered rock in north-east Scotland: Zeitschrift fur Geomorphologie N.F. Supplemental Bulletin, v. 72, p. 97-108.

Harbor, J.M. 1995, Development of glacial-valley cross sections under conditions of spatially variable resistance to erosion: Geomorphology, v. 14, p. 99-107.

Harbor, J.M. 1992, Numerical modeling of the development of U-shaped valleys by glacial erosion: Geological Society Bulletin, v. 104, p. 1364-1375.

Hallet, B. 1995, Glacial quarrying: a simple theoretical model: Annals of Glaciology, v. 22, p. 1-8.

Hay, T. 1943, Notes on Glacial Erosion and Stone Stripes: The Geographical Journal, v.102, p.13-20.

Hirano, M. and Aniya, M., 1988, A rational explanation of cross-profile morphology for glacial valleys and of glacial valley development: Earth Surface and Processes, v. 13, p. 707-716.

Hooyer, T.S, Cohen, D. and Iverson, N.R,. 2012, Control of glacial quarrying by bedrock joints: Geomorphology, v.153-154, p. 91-101. 
Horton, R.E., 1945, Erosional development of streams and their drainage basins; hydrophysical approach to quantitative morphology: Geological society of America bulletin, v. 56, p. 275-370.

Huber, N.K. and Rinehart, C.D., 1965, Geologic map of the Devils Postpile quadrangle, Sierra Nevada, California: U.S. Geological Survey, Geologic Quadrangle Map GQ-437, scale $1: 62,500,1$ sheet.

Iverson, N.R. 2012, A theory of glacial quarrying for landscape evolution models: Geology, v.40, p. 679-682.

Iverson, N.R. 1991, Potential effects of subglacial water pressure fluctuations on quarrying: Journal of Glaciology, v.36, p. 304-314.

James, L.A., 1996, Polynomial and Power Functions for Glacial Valley Cross-section Morphology: Earth Surface Processes and Landforms, v. 21, p. 413-432.

James, L.A. 2003b, Glacial erosion and geomorphology in the northwest Sierra Nevada, CA: Geomorphology, v.55, p. 283-303.

Krabbendam, M. and Glasser, N. 2011, Glacial erosion and bedrock properties in NW Scotland: Abrasion and quarrying, hardness and joint spacing: Geomorphology, v. 130, p. 374-383.

Kochel, R.C., and Piper, J.F. 1986, Morphology of large valleys on Hawaii: Evidence for groundwater sapping and comparisons with Martian valleys: Journal of Geophysical Research: Solid Earth (1978-2012), v. 91, p. E175-E192.

Kottek, M.,Grieser, J., Beck, C., Rudolf, B., and Rubel, F., 2006. World Map of the Koppen-Geiger climate classification updated: Meteorologische Zeitschrift, v. 15, p. 259263. 
Leith, K., Moore, J., Amann, F., and Loew, S. 2011, Effect of glacial ice cover on fracturing in critically stressed bedrock: Geophysical Research Abstracts, EGU General Assembly, v. 13, EGU2011-12825-2.

Loso, M.G., Anderson, R.S., Anderson, S.P., and Reimer, P.J., 2006, A 1500-year record of temperature and glacial response inferred from varved Iceberg Lake, southcentral Alaska: Quaternary Research, v.66, p. 12-24.

Li, Y., Liu, G., and Cui, Z. 2001, Glacial valley cross-profile morphology, Tian Shan Mountains, China: Geomorphology, v.38, p. 153-166.

MacGregor, K.R., Anderson, R.S., Anderson, S.P., and Waddington, E.D. 2000, Numerical simulations of glacial-valley longitudinal profile evolution: Geology, v. 28, p. 1031-1034.

Martel, S.J. 2006, Effect of topographic curvature on near-surface stresses and application to sheeting joints: Geophysical Research Letters, v.33, p. 1-5.

Matlab 8.3, Natick, Massachusetts, The Mathworks Inc., 2012.

Matthes, F.E. 1965, Glacial Reconnaissance of Sequoia National Park California: U.S. Geologic Survey Professional Paper 504-A, 164 pp.

Matthes, F.E. 1960, Reconnaissance of the Geomorphology and Glacial Geology of the San Joaquin Basin, Sierra Nevada California: U.S. Geologic Survey Professional Paper 329, 62 pp.

Matthes, F.E. 1930, Geologic History of the Yosemite Valley: U.S. Geologic Survey Professional Paper 160, 131 pp. 
Minnich, R.A. 2007, Climate, Paleoclimate and Paleovegetation in Barbour, M.G., Keeler-Wolf, T, and Schoenherr, A.S. eds., Terrestrial Vegetation of California: University of California Press, p. 43-70.

Moore, J. G., and Mack, G.S., 2008, Map Showing Limits of Tahoe Glaciation in Sequoia and Kings Canyon National Parks, California, scale 1:25,000, 1 sheet.

Moore, J.G., and Sisson, T.W., 1978, Geologic map of the Triple Divide Peak quadrangle, Tulare County, California: U.S. Geological Survey, Geologic Quadrangle Map GQ-1636, scale 1:62,500, 1 sheet.

Moore, J.R., Sanders, J.W., Dietrich, W.E., and Glasser, S.D., 2009, Influence of rock mass strength on the erosion rate of alpine cliffs: Earth Surface Processes and Landforms.

Morland, L.W., and Boulton, G.S. 1975, Stress in an elastic hump: the effects of glacier flow over elastic bedrock: Proceedings of the Royal Society of London Series A, v. 344, p.157-73.

Moscicki, J., Kotara, A., and Kedzia, S., 2006. Glacial erosion in the Abisko Mountains: Karevagge and Vassivagge, Northern Sweden: Geografiska Annaler, v. 88, p. 151-163.

Nicholas, A.P., and Quine, T.A., 2010, Quantitative assessment of landform equifinality and paleoenvironmental reconstruction using geomorphic models, Geomorphology, v. 121, p. 167-183.

Palmstrom, A. 2005, Measurements of and Correlations between Block Size and Rock Quality Designation (RQD): Tunnel and Underground Space Technology, v. 20, p. 362377.

Palmstrom, A. 1995, RMi - a rock mass characterization system for rock engineering purposes, PhD thesis, Oslo University, Norway, 400p. 
Phillips, J., 1997, Simplexity and the reinvention of equifinality: Geographical Analysis, v. 29, p. 1-15.

Potter, E.C. 1976, Pleistocene glaciation in Ethiopia: new evidence: Journal of Glaciology, v. 17, p. 148-150.

Rea, B.R. 1994, Joint Control in the Formation of Rock Steps in the Subglacial Environment, in Robinson, D.A. and Williams, R.G., eds., Rock Weathering and Landform Evolution: John Wiley \& Sons Ltd., p. 473-486.

Schaffer, J. 1997, The Geomorphic Evolution of the Yosemite Valley and Sierra Nevada Landscapes: Solving the Riddles in the Rocks: Berkeley, California, Wilderness Press, $388 \mathrm{p}$.

Segall, P., 1984, Formation and growth of extensional fracture sets: Geological Society of America Bulletin, v. 95, p. 454-462.

Segall, P., McKee, H., Martel, S.J., and Turrin, B.D., 1990, Late Cretaceous age of fractures in the Sierra Nevada batholith, California: Geology, v. 18, p. 1248-1251.

Selby, M.J. 1980, A rock mass strength classification for geomorphic purposes: with tests from Antarctica and New Zealand: Zeitschrift Geomorphologie, v. 24, p. 31-51.

Smith D.G. and Fisher T.G. 1993, Glacial Lake Agassiz: The northwestern outlet and paleoflood: Geology, v. 21, p. 9-12.

Sklar, L.S. and Dietrich, W.E. 2001, Sediment and rock strength controls on river incision into bedrock; Geology, v. 29, p. 1087-1090. 
Stock, GM., Martel, S.J., Collins, B.D., and Harp E.L., 2011, Progressive failure of sheeted rock slopes: the 2009-2010 Rhombus Wall rock falls in Yosemite Valley, California, USA: Earth Surface Processes and Landforms, v. 37, p. 546-561.

Yingkui, L., Gengnian L., and Zhijiu, C. 2001, Longitudinal variations in cross-section morphology along a glacier valley: a case-study from the Tien Shan, China: Journal of Glaciology, v. 47, p. 243-250.

White, W.A., 1945, Origin of Granite Domes in the Southeastern Piedmont: The Journal of Geology, v. 53, p. 276-282.

Ziegler, M., Loew, S., and Moore, J.R., 2013, Distribution and inferred ago of exfoliation joints in the Aar Granite of the central Swiss Alps and relationship to Quaternary landscape evolution: Geomorphology, v. 201, p. 344-362. 
APPENDIX A

\begin{tabular}{|c|c|c|c|c|c|c|c|c|}
\hline \multicolumn{9}{|c|}{ Tuolumne River valley } \\
\hline \multicolumn{9}{|l|}{\begin{tabular}{|l|} 
Waypoint \\
92 (T7)
\end{tabular}} \\
\hline Lat/Long & $\begin{array}{l}\text { Elev. } \\
\text { (m) }\end{array}$ & $\mathrm{SH}$ & Alpha & $\begin{array}{l}\text { Joint } \\
\text { spacing } \\
(\mathrm{m})\end{array}$ & $\begin{array}{l}\text { Joint } \\
\text { length } \\
\text { (m) }\end{array}$ & $\begin{array}{l}\text { Joint } \\
\text { width } \\
(\mathrm{mm})\end{array}$ & $\begin{array}{l}\text { Joint strike } \\
\text { and dip }\end{array}$ & Continuity \\
\hline $\begin{array}{l}37.93520 \\
119.4889\end{array}$ & 1758 & 60 & -45 & $r=28$ & $3.5(1)$ & na & 15 & $r=6$ \\
\hline & & 64 & & & $5(2)$ & na & 15 & \\
\hline & & 48 & & & $1.5(3)$ & na & 125,88 & \\
\hline & & 68 & & & $1(4)$ & 0.5 & 90,90 & \\
\hline & & 60 & & & $5(5)$ & 0.5 & 90,45 & \\
\hline & & 60 & & & & $r=7$ & $r=18$ & \\
\hline & & 48 & & & & & & \\
\hline & & 68 & & & & & & \\
\hline & & 64 & & & & & & \\
\hline & & 63 & & & & & & \\
\hline & & 65 & & & & & & \\
\hline & & 55 & & & & & & \\
\hline & & 63 & & & & & & \\
\hline & & 63 & & & & & & \\
\hline & & 63 & & & & & & \\
\hline & & 55 & & & & & & \\
\hline & & 63 & & & & & & \\
\hline & & 61 & & & & & & \\
\hline & & & & & & & & \\
\hline $\begin{array}{l}\text { Waypoint } \\
93\end{array}$ & & & & & & & & \\
\hline $\begin{array}{l}37.93506 \\
119.48881\end{array}$ & 1777 & 70( & -45 & 0.8 & $2.5(1)$ & 0.5 & 6 & $r=5$ \\
\hline & & 65 & & 0.3 & $3(2)$ & 0.5 & 125,89 & \\
\hline & & 66 & & 0.3 & $>5(3)$ & na & 10 & \\
\hline & & 69 & & 3 & $>5(4)$ & na & 68 & \\
\hline & & 73 & & 2 & $2(5)$ & 2.5 & 75,60 (flake & \\
\hline & & 68 & & 0.6 & & $r=4$ & $r=9$ & \\
\hline & & 64 & & 0.7 & & & & \\
\hline & & 71 & & $r=21$ & & & & \\
\hline
\end{tabular}




\begin{tabular}{|c|c|c|c|c|c|c|c|c|}
\hline & & 69 & & & & & & \\
\hline & & 70 & & & & & & \\
\hline & & 69 & & & & & & \\
\hline & & 64 & & & & & & \\
\hline & & 64 & & & & & & \\
\hline & & 66 & & & & & & \\
\hline & & 68 & & & & & & \\
\hline & & 71 & & & & & & \\
\hline & & 72 & & & & & & \\
\hline & & 68 & & & & & & \\
\hline & & & & & & & & \\
\hline $\begin{array}{l}\text { Waypoint } \\
94\end{array}$ & & & & & & & & \\
\hline $\begin{array}{l}37.93491 \\
119.48875 \\
\end{array}$ & 1781 & $72(\mathrm{P}$ & -45 & 1 & $1.5(1)$ & face & 357,68 & \\
\hline & & 72 & & 2 & $3(2)$ & na & 4 & \\
\hline & & 72 & & 2.5 & $>6(3)$ & face & 65 & \\
\hline & & 70 & & 1.5 & $2(4)$ & 3 & 68 & \\
\hline & & 73 & & 2 & $2.5(5)$ & 2-Jan & 260,60 & \\
\hline & & 70 & & 3 & $3(6)$ & 2 & 240,60 & \\
\hline & & 73 & & 2 & $>5(7)$ & 4 & 247,55 & \\
\hline & & 71 & & $r=28$ & $6(8)$ & 3.5 & $263,7(?)$ & \\
\hline & & 74 & & & & $r=4$ & $r=18$ & $r=6$ \\
\hline & & 70 & & & & & & \\
\hline & & 72 & & & & & & \\
\hline & & 74 & & & & & & \\
\hline & & 64 & & & & & & \\
\hline & & 74 & & & & & & \\
\hline & & 68 & & & & & & \\
\hline & & 73 & & & & & & \\
\hline & & 70 & & & & & & \\
\hline & & 71 & & & & & & \\
\hline & & & & & & & & \\
\hline $\begin{array}{l}\text { Waypoint } \\
95\end{array}$ & & & & & & & & \\
\hline $\begin{array}{l}37.93622 \\
119.48849\end{array}$ & 1766 & 56 & -45 & 0.9 & $3(1)$ & 20 & 63,80 & $r=5$ \\
\hline & & 58 & & 0.6 & $>5(2)$ & 10 & 120 & \\
\hline
\end{tabular}




\begin{tabular}{|c|c|c|c|c|c|c|c|c|}
\hline & & 40 & & 0.9 & $1.5(3)$ & face & 15,73 & \\
\hline & & 60 & & 1 & $>5(4)$ & 60 & 140,10 & \\
\hline & & 58 & & 0.3 & $3(5)$ & 20 & 107,90 & \\
\hline & & 64 & & 0.6 & $2(6)$ & 60 & 170 & \\
\hline & & 58 & & 2 & & $r=2$ & $r=9$ & \\
\hline & & $\overline{59}$ & & 0.9 & & & & \\
\hline & & $\overline{59}$ & & $r=21$ & & & & \\
\hline & & 37 & & & & & & \\
\hline & & $\overline{50}$ & & & & & & \\
\hline & & 47 & & & & & & \\
\hline & & 58 & & & & & & \\
\hline & & 54 & & & & & & \\
\hline & & 59 & & & & & & \\
\hline & & 54 & & & & & & \\
\hline & & 52 & & & & & & \\
\hline & & 54 & & & & & & \\
\hline & & & & & & & & \\
\hline $\begin{array}{l}\text { Waypoint } \\
96\end{array}$ & & & & & & & & \\
\hline $\begin{array}{l}37.93616 \\
119.48857\end{array}$ & 1798 & 50 & -90 & 0.3 & $1.5(1)$ & face & 90,90 & $r=5$ \\
\hline & & $\overline{58}$ & & 0.3 & $0.5(2)$ & face & 60,90 & \\
\hline & & 54 & & 0.6 & $2(3)$ & face & 80,90 & \\
\hline & & 54 & & 0.5 & 0.5 (4) & 4 & 57 & \\
\hline & & 52 & & 0.4 & & $r=7$ & $r=14$ & \\
\hline & & 56 & & $r=21$ & & & & \\
\hline & & 54 & & & & & & \\
\hline & & 54 & & & & & & \\
\hline & & 56 & & & & & & \\
\hline & & 58 & & & & & & \\
\hline & & 56 & & & & & & \\
\hline & & 51 & & & & & & \\
\hline & & 50 & & & & & & \\
\hline & & 60 & & & & & & \\
\hline & & 58 & & & & & & \\
\hline & & 58 & & & & & & \\
\hline & & 61 & & & & & & \\
\hline & & 54 & & & & & & \\
\hline & & $\mathbf{5 5}$ & & & & & & \\
\hline
\end{tabular}




\begin{tabular}{|c|c|c|c|c|c|c|c|}
\hline $\begin{array}{l}\text { Waypoint } \\
97 \text { (T6) }\end{array}$ & & & & & & & \\
\hline $\begin{array}{l}37.93097 \\
119.51716\end{array}$ & 1684 & -90 & $r=28$ & $\begin{array}{l}>5 \mathrm{~m} \\
(1)\end{array}$ & na & 32 & $r=7$ \\
\hline & & 58 & & $1(2)$ & na & 40 & \\
\hline & & 56 & & $5(3)$ & na & 27 & \\
\hline & & 52 & & $2.5(4)$ & na & 135 & \\
\hline & & 64 & & $2(5)$ & na & 135 & \\
\hline & & 64 & & $\begin{array}{l}>5 \mathrm{~m} \\
(6)\end{array}$ & na & 27 & \\
\hline & & 66 & & & $r=7$ & $r=20$ & \\
\hline & & 50 & & & & & \\
\hline & & 61 & & & & & \\
\hline & & 66 & & & & & \\
\hline & & 63 & & & & & \\
\hline & & 63 & & & & & \\
\hline & & 65 & & & & & \\
\hline & & 61 & & & & & \\
\hline & & 66 & & & & & \\
\hline & & 68 & & & & & \\
\hline & & 72 & & & & & \\
\hline & & 62 & & & & & \\
\hline & & & & & & & \\
\hline $\begin{array}{l}\text { Waypoint } \\
98\end{array}$ & & & & & & & \\
\hline $\begin{array}{l}37.93122 \\
119.51714\end{array}$ & 1695 & 72 (P-90 & $r=28$ & $2.5(1)$ & na & 60 & $r=7$ \\
\hline & & 72 & & $2(2)$ & na & 60 & \\
\hline & & 70 & & $4(3)$ & na & 10 & \\
\hline & & 75 & & $>4(4)$ & na & 10 & \\
\hline & & 72 & & & $r=7$ & $r=20$ & \\
\hline & & 71 & & & & & \\
\hline & & 76 & & & & & \\
\hline & & 68 & & & & & \\
\hline & & 72 & & & & & \\
\hline & & 72 & & & & & \\
\hline & & 73 & & & & & \\
\hline & & 70 & & & & & \\
\hline
\end{tabular}




\begin{tabular}{|c|c|c|c|c|c|c|c|c|}
\hline & & 70 & & & & & & \\
\hline & & 75 & & & & & & \\
\hline & & 72 & & & & & & \\
\hline & & 72 & & & & & & \\
\hline & & 68 & & & & & & \\
\hline & & 72 & & & & & & \\
\hline & & & & & & & & \\
\hline \multicolumn{9}{|l|}{$\begin{array}{l}\text { Waypoint } \\
99\end{array}$} \\
\hline \multirow[t]{19}{*}{$\begin{array}{l}37.93180 \\
119.51706 \\
\end{array}$} & 1720 & 52 & -90 & & $2(1)$ & 30 & 55 & $r=5$ \\
\hline & & 64 & & & $0.5(2)$ & 5 & 50 & \\
\hline & & 66 & & & $0.5(3)$ & 5 & 130 & \\
\hline & & 65 & & & $>4(4)$ & 10 & 110 & \\
\hline & & 55 & & & $3-4(5)$ & 15 & 169 & \\
\hline & & 58 & & & $3(6)$ & 20 & 5 & \\
\hline & & 66 & & & $2(7)$ & 30 & 55 & \\
\hline & & 62 & & & $3.5(8)$ & 60 & 115 & \\
\hline & & 58 & & & & & $r=14$ & \\
\hline & & 58 & & $r=21$ & & $r=2$ & & \\
\hline & & 63 & & & & & & \\
\hline & & 63 & & & & & & \\
\hline & & 65 & & & & & & \\
\hline & & 64 & & & & & & \\
\hline & & 52 & & & & & & \\
\hline & & 64 & & & & & & \\
\hline & & 58 & & & & & & \\
\hline & & 61 & & & & & & \\
\hline & & & & & & & & \\
\hline \multicolumn{9}{|l|}{$\begin{array}{l}\text { Waypoint } \\
100\end{array}$} \\
\hline $\begin{array}{l}37.931217 \\
119.51713\end{array}$ & 1766 & $68(\mathrm{P}$ & -90 & & $5.5(1)$ & $3 \mathrm{~mm}$ & 347 & $r=6$ \\
\hline & & 66 & & & $2(2)$ & face & 350,85 & \\
\hline & & 70 & & & $>5(3)$ & $1 \mathrm{~mm}$ & 76 & \\
\hline & & 64 & & & $>4(4)$ & na & 62 & \\
\hline
\end{tabular}




\begin{tabular}{|c|c|c|c|c|c|c|c|c|}
\hline & & 68 & & & $3(5)$ & na & 145 & \\
\hline & & 62 & & $r=21$ & & $r=5$ & $r=18$ & \\
\hline & & 66 & & & & & & \\
\hline & & 70 & & & & & & \\
\hline & & 62 & & & & & & \\
\hline & & 66 & & & & & & \\
\hline & & 64 & & & & & & \\
\hline & & 64 & & & & & & \\
\hline & & 67 & & & & & & \\
\hline & & 68 & & & & & & \\
\hline & & 63 & & & & & & \\
\hline & & 60 & & & & & & \\
\hline & & 62 & & & & & & \\
\hline & & 65 & & & & & & \\
\hline & & & & & & & & \\
\hline $\begin{array}{l}\text { Waypoint } \\
101 \text { (T5) }\end{array}$ & & & & & & & & \\
\hline $\begin{array}{l}37.93867 \\
119.53250 \\
\end{array}$ & 1619 & 55 & -90 & & $>5(1)$ & $4 \mathrm{~mm}$ & 135,90 & \\
\hline & & 55 & & & $0.5(2)$ & $1 \mathrm{~mm}$ & 40,34 & \\
\hline & & 66 & & & $0.5(3)$ & na & 78,42 & \\
\hline & & 62 & & & $1(4)$ & $1 \mathrm{~mm}$ & 48,40 & \\
\hline & & 58 & & & $0.5(5)$ & na & 85,30 & \\
\hline & & 60 & & & $\begin{array}{l}>10(6) \\
\& 5 \\
(10)\end{array}$ & $5 \mathrm{~mm}$ & 63,90 & \\
\hline & & 66 & & & $1(7)$ & $1 \mathrm{~mm}$ & 27,45 & \\
\hline & & 68 & & & $>6(8)$ & na & 343,86 & \\
\hline & & 59 & & $r=28$ & & $r=6$ & $r=14$ & $r=6$ \\
\hline & & 61 & & & & & & \\
\hline & & 64 & & & & & & \\
\hline & & 58 & & & & & & \\
\hline & & 65 & & & & & & \\
\hline & & 64 & & & & & & \\
\hline & & 61 & & & & & & \\
\hline & & 65 & & & & & & \\
\hline & & 63 & & & & & & \\
\hline & & 62 & & & & & & \\
\hline
\end{tabular}




\begin{tabular}{|c|c|c|c|c|c|c|c|c|}
\hline $\begin{array}{l}\text { Waypoint } \\
102\end{array}$ & & & & & & & & \\
\hline $\begin{array}{l}37.93851 \\
119.53333 \\
\end{array}$ & 1583 & 56 & 0 & 0.2 & $\begin{array}{l}0.6 \text { (set } \\
1)\end{array}$ & na & 130,90 & \\
\hline & & 58 & & 2 & $\begin{array}{l}7 \text { (vein } \\
1)\end{array}$ & 2 & 90 & \\
\hline & & 49 & & 0.6 & $\begin{array}{l}0.5 \text { (set } \\
2)\end{array}$ & 1.5 & 70,57 & \\
\hline & & 58 & & 1 & $\begin{array}{l}3 \\
\text { (veins } \\
2 \text { ) }\end{array}$ & $2 \mathrm{~mm}$ & 65 & \\
\hline & & 56 & & 2 & $>3(3)$ & $3.5 \mathrm{~mm}$ & 140 & \\
\hline & & 58 & & 1 & $>3(4)$ & $9 \mathrm{~mm}$ & 15 & \\
\hline & & 58 & & $r=28$ & \begin{tabular}{|l}
1.6 \\
(vein \\
3 )
\end{tabular} & 1 & 72 & \\
\hline & & 59 & & & $\begin{array}{l}>3.5 \\
(5)\end{array}$ & na & 80,90 & \\
\hline & & 65 & & & $\begin{array}{l}1.8(6) \\
\text { Flake(? }\end{array}$ & $1 \mathrm{~mm}$ & 130,86 & \\
\hline & & 66 & & & $\begin{array}{l}>4 \\
\text { (vein4) }\end{array}$ & 3 & $130(?), 34$ & \\
\hline & & 60 & & & & $r=6$ & $r=9$ & $r=5$ \\
\hline & & 50 & & & & & & \\
\hline & & 52 & & & & & & \\
\hline & & 56 & & & & & & \\
\hline & & 56 & & & & & & \\
\hline & & 62 & & & & & & \\
\hline & & 64 & & & & & & \\
\hline & & 58 & & & & & & \\
\hline $\begin{array}{l}\text { Waypoint } \\
103 \text { (T4) }\end{array}$ & & & & & & & & \\
\hline $\begin{array}{l}37.93404 \\
119.55695\end{array}$ & 1489 & 54 & -90 & 0.4 & $0.5(1)$ & $3 \mathrm{~mm}$ & 134,80 & $r=5$ \\
\hline & & 52 & & 0.6 & $1.5(2)$ & na & 55 & \\
\hline & & 46 & & 0.6 & $0.5(3)$ & na & 137,80 & \\
\hline
\end{tabular}




\begin{tabular}{|c|c|c|c|c|c|c|c|c|}
\hline & & 46 & & 0.9 & $\begin{array}{l}1.5(4) \\
2 \mathrm{nd} / / \\
\text { joint } \\
1 \mathrm{~m} .\end{array}$ & 2 & 220,85 & \\
\hline & & 54 & & & $\begin{array}{l}>2(5) \\
\text { Four // } \\
\text { joints } \\
1 \mathrm{~m}\end{array}$ & 1 & 134 & \\
\hline & & 55 & & & $16(6)$ & 2 & 5,87 & \\
\hline & & 54 & & & $\begin{array}{l}2(7) \\
2 \mathrm{nd} / / \\
\text { joint } \\
10 \mathrm{~cm} .\end{array}$ & 4 & 235 & \\
\hline & & 51 & & 0.6 & & $r=4$ & & \\
\hline & & 46 & & $r=21$ & & & $r=9$ & \\
\hline & & 51 & & & & & & \\
\hline & & 50 & & & & & & \\
\hline & & 46 & & & & & & \\
\hline & & 44 & & & & & & \\
\hline & & 48 & & & & & & \\
\hline & & 50 & & & & & & \\
\hline & & 48 & & & & & & \\
\hline & & 50 & & & & & & \\
\hline & & & & & & & & \\
\hline $\begin{array}{l}\text { Waypoint } \\
104\end{array}$ & & & & & & & & \\
\hline $\begin{array}{l}37.93425 \\
119.55743\end{array}$ & 1507 & 60 & -90 & 0.3 & $\begin{array}{l}3 \text { (Set } \\
1 \text { ) }\end{array}$ & 127 & 84,52 & $r=5$ \\
\hline & & 54 & & 0.3 & $\begin{array}{l}1 \text { (Set } \\
2 \text { ) }\end{array}$ & 150 & 342,67 & \\
\hline & & 53 & & 0.6 & $\begin{array}{l}3.5 \\
\text { (Set3) }\end{array}$ & 55 & 85,65 & \\
\hline & & 46 & & 0.9 & & $r=2$ & $r=14$ & \\
\hline & & 48 & & 0.45 & & & & \\
\hline & & 63 & & $r=21$ & & & & \\
\hline & & 65 & & & & & & \\
\hline & & 54 & & & & & & \\
\hline & & 50 & & & & & & \\
\hline & & 48 & & & & & & \\
\hline
\end{tabular}




\begin{tabular}{|c|c|c|c|c|c|c|c|c|}
\hline & & 56 & & & & & & \\
\hline & & 58 & & & & & & \\
\hline & & 58 & & & & & & \\
\hline & & 66 & & & & & & \\
\hline & & 62 & & & & & & \\
\hline & & 60 & & & & & & \\
\hline & & 54 & & & & & & \\
\hline & & 56 & & & & & & \\
\hline & & & & & & & & \\
\hline \multicolumn{9}{|l|}{$\begin{array}{l}\text { Waypoint } \\
105\end{array}$} \\
\hline \multirow[t]{19}{*}{$\begin{array}{l}37.93434 \\
119.55788 \\
\end{array}$} & 1509 & 69 & -90 & & $>4(1)$ & na & 17,78 & $r=5$ \\
\hline & & 72 & & & $>3(2)$ & na & 60 & \\
\hline & & 70 & & & $0.5(3)$ & na & 320 & \\
\hline & & 66 & & & $0.5(4)$ & na & 305 & \\
\hline & & 68 & & & $1.5(5)$ & 20 & 290 & \\
\hline & & 68 & & & $2(6)$ & 50 & 330,90 & \\
\hline & & 68 & & & $2.5(7)$ & 20 & 50 & \\
\hline & & 71 & & & $>2(8)$ & 30 & 320 & \\
\hline & & 68 & & & $1(9)$ & 25 & 30,40 & \\
\hline & & 70 & & & & $r=2$ & $r=14$ & \\
\hline & & 70 & & $r=28$ & & & & \\
\hline & & 70 & & & & & & \\
\hline & & 68 & & & & & & \\
\hline & & 68 & & & & & & \\
\hline & & 70 & & & & & & \\
\hline & & 68 & & & & & & \\
\hline & & 67 & & & & & & \\
\hline & & 69 & & & & & & \\
\hline & & & & & & & & \\
\hline \multicolumn{9}{|l|}{$\begin{array}{l}\text { Waypoint } \\
106 \text { (T3) }\end{array}$} \\
\hline $\begin{array}{l}37.92962 \\
119.56202\end{array}$ & 1459 & 58 & -90 & & $2(1)$ & $1 \mathrm{~mm}$ & 40,90 & $r=5$ \\
\hline & & 61 & & & $2.5(2)$ & $1.5 \mathrm{~mm}$ & 41,90 & \\
\hline & & 62 & & & $2.5(3)$ & $1 \mathrm{~mm}$ & 40,90 & \\
\hline & & 62 & & & $3(4)$ & $1.5 \mathrm{~mm}$ & 40,86 & \\
\hline
\end{tabular}




\begin{tabular}{|c|c|c|c|c|c|c|c|c|}
\hline & & 63 & & & $1.5(5)$ & na & 280 & \\
\hline & & 62 & & & $>3(6)$ & $1 \mathrm{~mm}$ & 40,90 & \\
\hline & & 53 & & $r=28$ & $>3(7)$ & na & 357,72 & \\
\hline & & 66 & & & $2(8)$ & na & 355,70 & \\
\hline & & 61 & & & \begin{tabular}{|l|}
$3(9)$ \\
\end{tabular} & na & \begin{tabular}{|l|}
281 \\
\end{tabular} & \\
\hline & & 54 & & & $\begin{array}{l}.5 \\
(10)\end{array}$ & na & 350 & \\
\hline & & 60 & & & $1(11)$ & $2 \mathrm{~mm}$ & 75,77 & \\
\hline & & 58 & & & \begin{tabular}{|l}
1.5 \\
$(12)$
\end{tabular} & $2 \mathrm{~mm}$ & 75,80 & \\
\hline & & 64 & & & $\begin{array}{l}0.5 \\
(13)\end{array}$ & $1 \mathrm{~mm}$ & 60,90 & \\
\hline & & 58 & & & $\begin{array}{l}1.8 \\
(14)\end{array}$ & $1 \mathrm{~mm}$ & 60,90 & \\
\hline & & 64 & & & \begin{tabular}{|l|}
0.5 \\
$(15)$ \\
\end{tabular} & $1 \mathrm{~mm}$ & 95,67 & \\
\hline & & 65 & & & \begin{tabular}{|l|}
0.5 \\
$(16)$
\end{tabular} & $1 \mathrm{~mm}$ & 95,68 & \\
\hline & & 52 & & & \begin{tabular}{|l|}
0.8 \\
$(17)$
\end{tabular} & $2 \mathrm{~mm}$ & 40 & \\
\hline & & 60 & & & \begin{tabular}{|l|}
0.8 \\
$(18)$ \\
\end{tabular} & $2 \mathrm{~mm}$ & 40 & \\
\hline & & & & & 1 (19) & na & 90,80 & \\
\hline & & & & & $1(20)$ & na & 70,80 & \\
\hline & & & & & \begin{tabular}{|l}
0.8 \\
$(21)$ \\
\end{tabular} & na & 60,80 & \\
\hline & & & & & $>3(22)$ & $6 \mathrm{~mm}$ & 355,85 & \\
\hline & & & & & $4(23)$ & na & 352 & \\
\hline & & & & & $>4(24)$ & face & 30,73 & \\
\hline & & & & & & $r=5$ & $r=14$ & \\
\hline & & & & & & & & \\
\hline $\begin{array}{l}\text { Waypoint } \\
107\end{array}$ & & & & & & & & \\
\hline $\begin{array}{l}37.92984 \\
119.56216\end{array}$ & 1477 & $54(\mathrm{P}$ & -90 & $r=28$ & $>4(1)$ & na & 45,50 & $r=7$ \\
\hline & & 66 & & & $>2(2)$ & na & 46,55 & \\
\hline & & 65 & & & $>3(3)$ & na & 43,50 & \\
\hline
\end{tabular}




\begin{tabular}{|c|c|c|c|c|c|c|c|c|}
\hline & & 66 & & & $0.3(4)$ & na & 320 & \\
\hline & & 69 & & & $\begin{array}{l}2 \text { (vein } \\
5)\end{array}$ & 2 & 355 & \\
\hline & & 68 & & & $\begin{array}{l}>2( \\
\text { vein 6) }\end{array}$ & $4 \mathrm{~mm}$ & 290 & \\
\hline & & 66 & & & $1(7)$ & 1 & 170 & \\
\hline & & 65 & & & & $r=7$ & $r=20$ & \\
\hline & & 66 & & & & & & \\
\hline & & 68 & & & & & & \\
\hline & & 70 & & & & & & \\
\hline & & 72 & & & & & & \\
\hline & & 74 & & & & & & \\
\hline & & 73 & & & & & & \\
\hline & & 75 & & & & & & \\
\hline & & 75 & & & & & & \\
\hline & & 70 & & & & & & \\
\hline & & 69 & & & & & & \\
\hline & & & & & & & & \\
\hline $\begin{array}{l}\text { Waypoint } \\
108\end{array}$ & & & & & & & & \\
\hline $\begin{array}{l}37.93003 \\
119.56244 \\
\end{array}$ & 1488 & 40 & 0 & $r=28$ & $>7(1)$ & face & 48,90 & $r=6$ \\
\hline & & 50 & & & $1(2)$ & $2 \mathrm{~mm}$ & 130,78 & \\
\hline & & 64 & & & $?(3)$ & Look at $p$ & See & \\
\hline & & 63 & & & & $r=5$ & $r=18$ & \\
\hline & & 58 & & & & & & \\
\hline & & 56 & & & & & & \\
\hline & & 54 & & & & & & \\
\hline & & 54 & & & & & & \\
\hline & & 60 & & & & & & \\
\hline & & 59 & & & & & & \\
\hline & & 61 & & & & & & \\
\hline & & 50 & & & & & & \\
\hline & & 51 & & & & & & \\
\hline & & 64 & & & & & & \\
\hline & & 64 & & & & & & \\
\hline & & 58 & & & & & & \\
\hline & & 59 & & & & & & \\
\hline & & 57 & & & & & & \\
\hline
\end{tabular}




\begin{tabular}{|l|l|l|l|l|l|l|l|l|}
\hline $\begin{array}{l}\text { Waypoint } \\
\mathbf{1 0 9} \text { (T2) }\end{array}$ & & & & & & & & \\
\hline $\begin{array}{l}37.93203 \\
119.59145\end{array}$ & 1333 & 56 & -45 & & $3(1)$ & face & 190,83 & $\mathbf{r}=\mathbf{6}$ \\
\hline & & 68 & & & $1.5(2)$ & vein & 68 & \\
\hline & & 52 & & & $2(3)$ & vein & 24 & \\
\hline & & 58 & & & $3(4)$ & na & 330,78 & \\
\hline & & 59 & & & $3(5)$ & na & 247,73 & \\
\hline & & 64 & & $r=21$ & $2(6)$ & na & 238,77 & \\
\hline & & 60 & & & $>3(7)$ & $1 \mathrm{~mm}$ & 307 & \\
\hline & & 54 & & & $1.5(8)$ & na & 240,75 & \\
\hline & & 64 & & & $2.5(9)$ & face & 324,75 & \\
\hline & & 62 & & & $2(10)$ & na & 260,20 & \\
\hline & & 50 & & & & $r=6$ & $r=18$ & \\
\hline & & 60 & & & & & & \\
\hline & & 56 & & & & & & \\
\hline & & 60 & & & & & & \\
\hline & & 53 & & & & & & \\
\hline & & 61 & & & & & & \\
\hline & & 62 & & & & & & \\
\hline & & $\mathbf{5 9}$ & & & & & & \\
\hline & & & & & & & & \\
\hline & & & & & & & & \\
\hline & & & & & & & & \\
\hline & & & & & & & & \\
\hline & & & & & & & \\
\hline & & & & & & \\
\hline \\
\hline
\end{tabular}




\begin{tabular}{|c|c|c|c|c|c|c|c|c|}
\hline & & 63 & & & & & & \\
\hline & & 50 & & & & & & \\
\hline & & 57 & & & & & & \\
\hline & & 58 & & & & & & \\
\hline & & 58 & & & & & & \\
\hline & & & & & & & & \\
\hline \multicolumn{9}{|l|}{\begin{tabular}{|l|} 
Waypoint \\
111 (T1) \\
\end{tabular}} \\
\hline \multirow[t]{19}{*}{$\begin{array}{l}37.93018 \\
119.60405 \\
\end{array}$} & 1371 & 54 & -90 & & $0.5(1)$ & 3 & 355,47 & $r=6$ \\
\hline & & 48 & & & $2(2)$ & na & 15 & \\
\hline & & 56 & & & $>4(3)$ & na & 35 & \\
\hline & & 54 & & & $1.5(4)$ & 25 & 12,62 & \\
\hline & & 61 & & $r=15$ & \begin{tabular}{|l|}
$>3.5$ \\
$(5)$ \\
\end{tabular} & 25 & 10,60 & \\
\hline & & 60 & & & $0.5(6)$ & 1 & 14 & \\
\hline & & 62 & & & $3(7)$ & 1.5 & 350 & \\
\hline & & 64 & & & $3.5(8)$ & 20 & 9,75 & \\
\hline & & 64 & & & \begin{tabular}{|l|}
$1.5(9)$ \\
\end{tabular} & 15 & 95 & \\
\hline & & 63 & & & $4(10)$ & face & 223,60 & \\
\hline & & 63 & & & $2(11)$ & na & 235 & \\
\hline & & 59 & & & $>2(12)$ & 20 & 107,75 & \\
\hline & & 64 & & & & $r=2$ & $r=18$ & \\
\hline & & 60 & & & & & & \\
\hline & & 57 & & & & & & \\
\hline & & 66 & & & & & & \\
\hline & & 64 & & & & & & \\
\hline & & 60 & & & & & & \\
\hline & & & & & & & & \\
\hline \multicolumn{9}{|l|}{\begin{tabular}{|l|} 
Waypoint \\
112 \\
\end{tabular}} \\
\hline $\begin{array}{l}37.93072 \\
119.60443\end{array}$ & 1376 & 64 & -90 & $r=15$ & $\begin{array}{l}1 \text { (Set } \\
1 \text { ) }\end{array}$ & 2 & 147,86 & $r=5$ \\
\hline & & 62 & & & $\begin{array}{l}2 \text { (Set } \\
2)\end{array}$ & 1 & 57,90 & \\
\hline & & 66 & & & $\begin{array}{l}1.5 \\
\text { Set 3) }\end{array}$ & 1 & 15,74 & \\
\hline
\end{tabular}




\begin{tabular}{|c|c|c|c|c|c|c|c|c|}
\hline & & 70 & & & & $r=2$ & $r=14$ & \\
\hline & & 68 & & & & & & \\
\hline & & 44 & & & & & & \\
\hline & & 64 & & & & & & \\
\hline & & 66 & & & & & & \\
\hline & & 70 & & & & & & \\
\hline & & 68 & & & & & & \\
\hline & & 66 & & & & & & \\
\hline & & 66 & & & & & & \\
\hline & & 60 & & & & & & \\
\hline & & 74 & & & & & & \\
\hline & & 64 & & & & & & \\
\hline & & 70 & & & & & & \\
\hline & & 71 & & & & & & \\
\hline & & 65 & & & & & & \\
\hline & & & & & & & & \\
\hline $\begin{array}{l}\text { Waypoint } \\
113\end{array}$ & & & & & & & & \\
\hline $\begin{array}{l}37.93131 \\
119.60491\end{array}$ & 1377 & 64 & 0 & $r=21$ & $2(1)$ & 40 & 340,70 & $r=5$ \\
\hline & & 66 & & & $0.5(2)$ & face & 260,77 & \\
\hline & & 66 & & & $0.6(3)$ & 50 & 345,70 & \\
\hline & & 64 & & & $2(4)$ & 30 & 335,80 & \\
\hline & & 68 & & & $3(5)$ & 120 & 250,60 & \\
\hline & & 68 & & & $2.5(6)$ & 150 & 240,30 & \\
\hline & & 67 & & & $\begin{array}{l}>3.5 \\
(7)\end{array}$ & 60 & 265,70 & \\
\hline & & 62 & & & $>4(8)$ & 90 & 260,65 & \\
\hline & & 68 & & & & $r=2$ & $r=14$ & \\
\hline & & 66 & & & & & & \\
\hline & & 62 & & & & & & \\
\hline & & 63 & & & & & & \\
\hline & & 68 & & & & & & \\
\hline & & 61 & & & & & & \\
\hline & & 66 & & & & & & \\
\hline & & 67 & & & & & & \\
\hline & & 66 & & & & & & \\
\hline & & 65 & & & & & & \\
\hline
\end{tabular}




\begin{tabular}{|c|c|c|c|c|c|c|c|c|}
\hline \multicolumn{9}{|c|}{ Tenaya Canyon } \\
\hline \multicolumn{9}{|c|}{\begin{tabular}{l|l} 
Waypoint & \\
? (TN8) &
\end{tabular}} \\
\hline Lat/Long & $\begin{array}{l}\text { Elev. } \\
\text { (m) }\end{array}$ & $\mathrm{SH}$ & Alpha & $\begin{array}{l}\text { Joint } \\
\text { length } \\
\text { (m) }\end{array}$ & $\begin{array}{l}\text { Joint } \\
\text { width } \\
(\mathrm{mm})\end{array}$ & $\begin{array}{l}\text { Joint } \\
\text { strike and } \\
\text { dip }\end{array}$ & \begin{tabular}{|l} 
Joint \\
spacing \\
(m)
\end{tabular} & Continuity \\
\hline $\begin{array}{l}37.80755 \\
119.47040\end{array}$ & & 60 & -90 & $>10$ & 100 & 185,65 & 3.5 & $r=6$ \\
\hline & & 52 & & 3.5 & 10 & 105,82 & 0.75 & \\
\hline & & 58 & & & 40 & $r=9$ & 1.25 & \\
\hline & & 48 & & & 50 & & 1 & \\
\hline & & 50 & & & 15 & & 0.4 & \\
\hline & & 40 & & & 40 & & 0.3 & \\
\hline & & 54 & & & 100 & & 0.4 & \\
\hline & & 50 & & & 40 & & 2 & \\
\hline & & 56 & & & $r=2$ & & 0.875 & \\
\hline & & 54 & & & & & $r=21$ & \\
\hline & & 52 & & & & & & \\
\hline & & 50 & & & & & & \\
\hline & & 5 & & & & & & \\
\hline & & 40 & & & & & & \\
\hline & & 52 & & & & & & \\
\hline & & 43 & & & & & & \\
\hline & & 5 & & & & & & \\
\hline & & 51 & & & & & & \\
\hline & & & & & & & & \\
\hline $\begin{array}{l}\text { Waypoint } \\
204\end{array}$ & & 63 & & & & & & \\
\hline $\begin{array}{l}37.80739 \\
119.47012\end{array}$ & & 60 & -90 & & 10 & 210,25 & 1.4 & $r=6$ \\
\hline & & 58 & & & 5 & 150,20 & 0.9 & \\
\hline & & 58 & & & 10 & $r=9$ & 5 & \\
\hline & & 64 & & & $r=4$ & & 4.5 & \\
\hline & & 64 & & & & & 2.95 & \\
\hline & & 58 & & & & & $r=28$ & \\
\hline & & 58 & & & & & & \\
\hline & & 58 & & & & & & \\
\hline & & 59 & & & & & & \\
\hline & & 60 & & & & & & \\
\hline
\end{tabular}




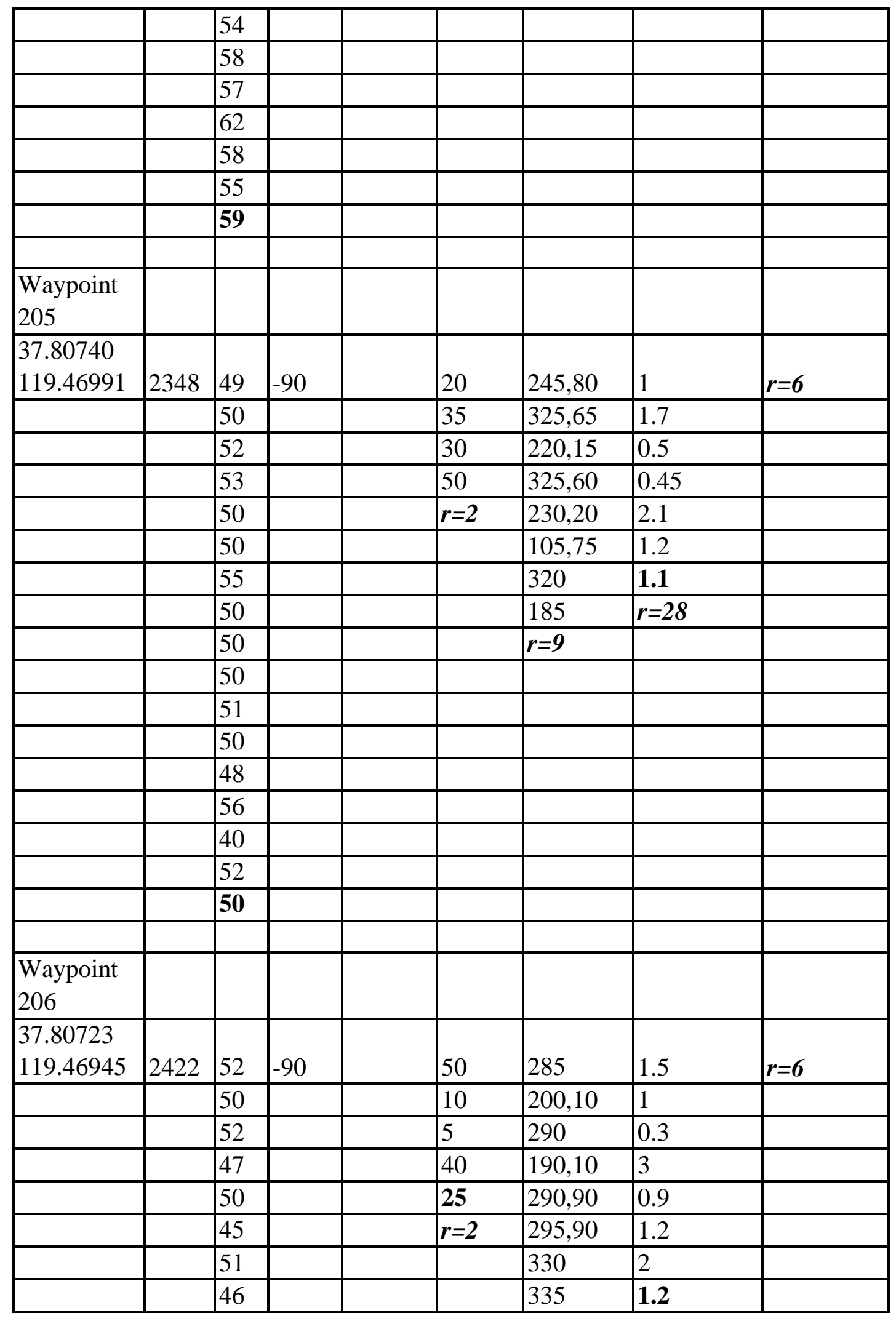




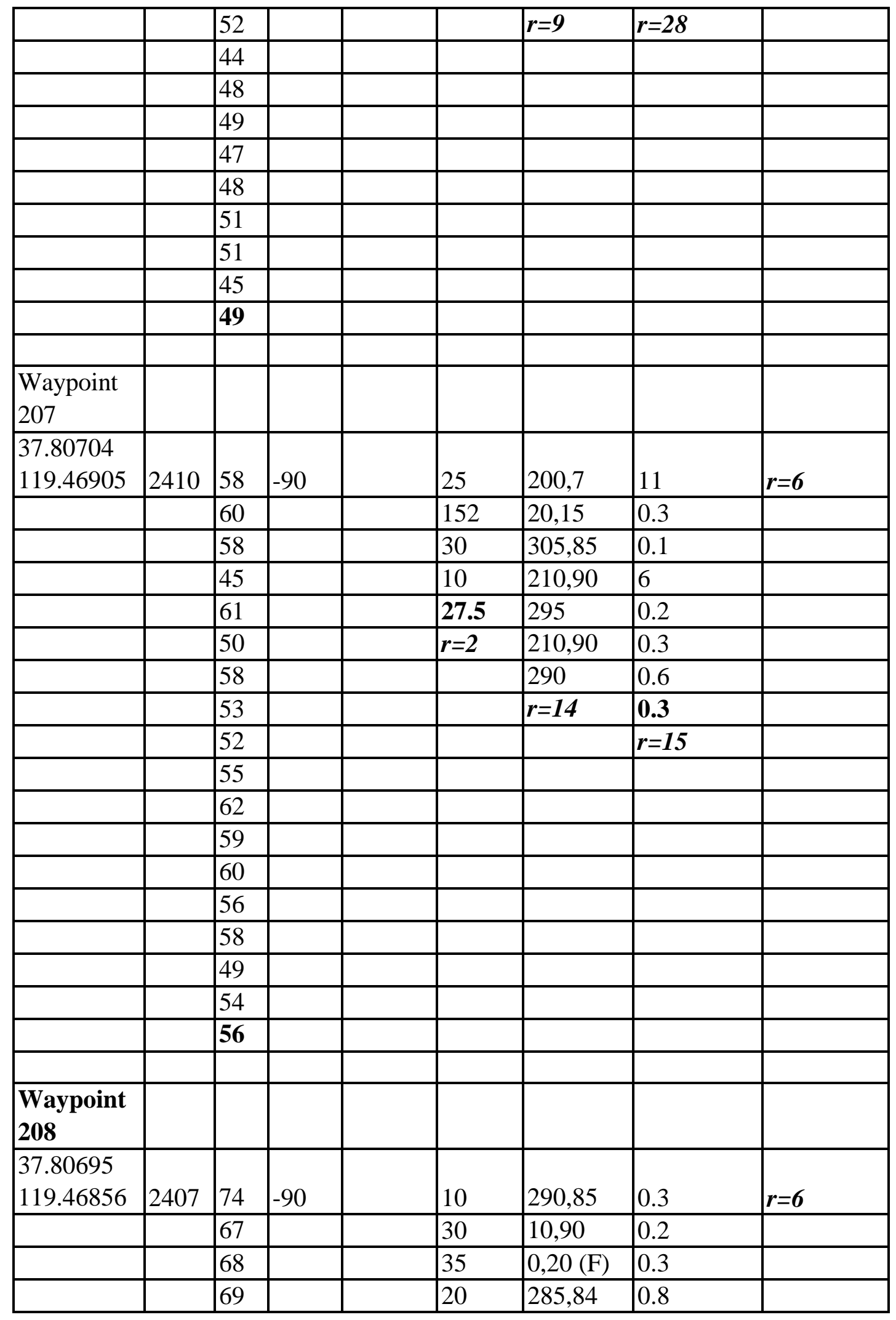




\begin{tabular}{|c|c|c|c|c|c|c|c|}
\hline & & 66 & & 10 & 292,79 & 0.3 & \\
\hline & & 64 & & 32 & 288,86 & 0.5 & \\
\hline & & 68 & & 25 & 285,90 & 0.25 & \\
\hline & & 70 & & $r=2$ & 10,88 & 1 & \\
\hline & & 68 & & & 15,86 & 3 & \\
\hline & & 70 & & & 12,90 & 2 & \\
\hline & & 68 & & & 290,88 & 1 & \\
\hline & & 70 & & & 289,86 & 1.5 & \\
\hline & & 70 & & & & 0.65 & \\
\hline & & 70 & & & & $r=15$ & \\
\hline & & 74 & & & & & \\
\hline & & 74 & & & & & \\
\hline & & 70 & & & & & \\
\hline & & 72 & & & & & \\
\hline & & 72 & & & & & \\
\hline & & 70 & & & & & \\
\hline & & & & & & & \\
\hline $\begin{array}{l}\text { Waypoint } \\
209\end{array}$ & & & & & & & \\
\hline $\begin{array}{l}37.80706 \\
119.46826\end{array}$ & 2420 & 48 & -45 & 10 & 105,90 & 0.8 & $r=6$ \\
\hline & & 67 & & 8 & 345 & 3.6 & \\
\hline & & 53 & & 40 & 125 & 1.8 & \\
\hline & & 66 & & $\sqrt{30}$ & 110,80 & 2.4 & \\
\hline & & 65 & & 15 & 10,82 & 2.1 & \\
\hline & & 58 & & 20 & 0,90 & 0.9 & \\
\hline & & 64 & & 25 & $0,10(\mathrm{~F})$ & 0.9 & \\
\hline & & 53 & & 20 & 145,85 & 1.8 & \\
\hline & & 63 & & $r=2$ & 280,83 & $r=28$ & \\
\hline & & 52 & & & & & \\
\hline & & 54 & & & & & \\
\hline & & 56 & & & & & \\
\hline & & 60 & & & & & \\
\hline & & 63 & & & & & \\
\hline & & 64 & & & & & \\
\hline & & 53 & & & & & \\
\hline & & 5 & & & & & \\
\hline & & & & & & & \\
\hline $\begin{array}{l}\text { Waypoint } \\
210\end{array}$ & & & & & & & \\
\hline
\end{tabular}




\begin{tabular}{|c|c|c|c|c|c|c|c|}
\hline \multirow[t]{2}{*}{$\begin{array}{l}37.80716 \\
119.46779 \\
\end{array}$} & \multirow[t]{2}{*}{2432} & 70 & -90 & 38 & 75,80 & $r=28$ & $r=7$ \\
\hline & & 65 & & 5 & 285,87 & & \\
\hline & & 58 & & 5 & $r=14$ & & \\
\hline & & 70 & & 10 & & & \\
\hline & & 69 & & 7.5 & & & \\
\hline & & 70 & & $\mathrm{r}=4$ & & & \\
\hline & & 70 & & & & & \\
\hline & & 68 & & & & & \\
\hline & & 74 & & & & & \\
\hline & & 66 & & & & & \\
\hline & & 72 & & & & & \\
\hline & & 67 & & & & & \\
\hline & & 70 & & & & & \\
\hline & & 70 & & & & & \\
\hline & & 66 & & & & & \\
\hline & & 70 & & & & & \\
\hline & & 75 & & & & & \\
\hline & & 69 & & & & & \\
\hline & & & & & & & \\
\hline $\begin{array}{l}\text { Waypoint } \\
211\end{array}$ & & & & & & & \\
\hline $\begin{array}{l}37.80735 \\
119.46729\end{array}$ & 2454 & 68 & -90 & 100 & 5,75 & 0.6 & $r=5$ \\
\hline & & 58 & & 20 & 265,70 & 0.3 & \\
\hline & & 68 & & 10 & $15,35(\mathrm{~F})$ & 0.3 & \\
\hline & & 66 & & 25 & 8,70 & 0.5 & \\
\hline & & 62 & & 10 & 5,85 & 0.7 & \\
\hline & & 60 & & 8 & 270,80 & 0.9 & \\
\hline & & 54 & & 115 & 265,73 & 1.2 & \\
\hline & & 66 & & 150 & $r=14$ & 0.3 & \\
\hline & & 52 & & 200 & & 0.15 & \\
\hline & & 52 & & 25 & & 0.2 & \\
\hline & & 63 & & 30 & & 0.2 & \\
\hline & & 58 & & 25 & & 0.9 & \\
\hline & & 66 & & $r=2$ & & 1.2 & \\
\hline & & 54 & & & & 0.5 & \\
\hline & & 64 & & & & $r=15$ & \\
\hline & & 58 & & & & & \\
\hline
\end{tabular}




\begin{tabular}{|c|c|c|c|c|c|c|c|}
\hline & & 54 & & & & & \\
\hline & & 60 & & & & & \\
\hline & & & & & & & \\
\hline \multicolumn{8}{|l|}{$\begin{array}{l}\text { Waypoint } \\
212 \text { (TN5) }\end{array}$} \\
\hline \multirow[t]{18}{*}{$\begin{array}{l}37.80617 \\
119.47073 \\
\end{array}$} & 2431 & 68 & -90 & 203 & 355,88 & 0.3 & 7 \\
\hline & & 64 & & 10 & 250 & 0.6 & \\
\hline & & 55 & & 30 & 275 & 0.15 & \\
\hline & & 67 & & 20 & 280 & 0.6 & \\
\hline & & 67 & & 15 & 260 & 0.5 & \\
\hline & & 68 & & 10 & 100,82 & 0.9 & \\
\hline & & 68 & & 12 & 325,80 & 0.9 & \\
\hline & & 65 & & 20 & 335 & 0.4 & \\
\hline & & 68 & & 30 & 320 & 0.2 & \\
\hline & & 69 & & 15 & 320,70 & 0.3 & \\
\hline & & 58 & & 25 & & 0.3 & \\
\hline & & 59 & & 30 & & 1.2 & \\
\hline & & 57 & & 150 & & 0.45 & \\
\hline & & 70 & & 120 & & & \\
\hline & & 60 & & 30 & & & \\
\hline & & 70 & & 40 & & & \\
\hline & & 58 & & 100 & & & \\
\hline & & 64 & & 30 & & & \\
\hline & & & & & & & \\
\hline \multicolumn{8}{|l|}{$\begin{array}{l}\text { Waypoint } \\
213\end{array}$} \\
\hline \multirow[t]{9}{*}{$\begin{array}{l}\text { No lat and } \\
\text { long data. } \\
\text { Next point } \\
\text { up. }\end{array}$} & 2419 & 65 & -90 & 50 & 325,65 & 1.8 & 5 \\
\hline & & 56 & & 30 & 325 & 4 & \\
\hline & & 64 & & 10 & 15 & 0.2 & \\
\hline & & 56 & & 20 & $\begin{array}{l}230,15 \\
\text { (F) }\end{array}$ & 2.1 & \\
\hline & & 60 & & 25 & & \begin{tabular}{|l|l}
1.2 \\
\end{tabular} & \\
\hline & & 58 & & & & 1.8 & \\
\hline & & 54 & & & & & \\
\hline & & 57 & & & & & \\
\hline & & 60 & & & & & \\
\hline
\end{tabular}




\begin{tabular}{|c|c|c|c|c|c|c|c|}
\hline & & 57 & & & & & \\
\hline & & 64 & & & & & \\
\hline & & 57 & & & & & \\
\hline & & 54 & & & & & \\
\hline & & 57 & & & & & \\
\hline & & 55 & & & & & \\
\hline & & 64 & & & & & \\
\hline & & 62 & & & & & \\
\hline & & 59 & & & & & \\
\hline & & & & & & & \\
\hline $\begin{array}{l}\text { Waypoint } \\
214\end{array}$ & & & & & & & \\
\hline $\begin{array}{l}37.80592 \\
119.46994\end{array}$ & 2403 & 65 & -90 & 10 & 310,90 & 0.6 & 6 \\
\hline & & 60 & & 40 & 308,84 & 0.3 & \\
\hline & & 64 & & 30 & 310,75 & 0.25 & \\
\hline & & 52 & & 8 & 05,86 & 0.9 & \\
\hline & & 56 & & 5 & $310(\mathrm{~V})$ & 0.2 & \\
\hline & & 57 & & 10 & $10(\mathrm{~V})$ & 0.3 & \\
\hline & & 61 & & & 320,88 & 1.2 & \\
\hline & & 60 & & & & 0.9 & \\
\hline & & 65 & & & & 0.2 & \\
\hline & & 64 & & & & 1.2 & \\
\hline & & 58 & & & & 0.15 & \\
\hline & & 66 & & & & 0.3 & \\
\hline & & 60 & & & & & \\
\hline & & 55 & & & & & \\
\hline & & 55 & & & & & \\
\hline & & 64 & & & & & \\
\hline & & 60 & & & & & \\
\hline & & 60 & & & & & \\
\hline & & & & & & & \\
\hline $\begin{array}{l}\text { Waypoint } \\
215\end{array}$ & & & & & & & \\
\hline $\begin{array}{l}37.80576 \\
119.46944\end{array}$ & 2386 & 62 & -45 & 0 & 195 & $>3$ & $R=7$ \\
\hline & & 58 & & $R=7$ & 191 & $R=30$ & \\
\hline & & 48 & & & 196 & & \\
\hline & & 56 & & & 195 & & \\
\hline
\end{tabular}




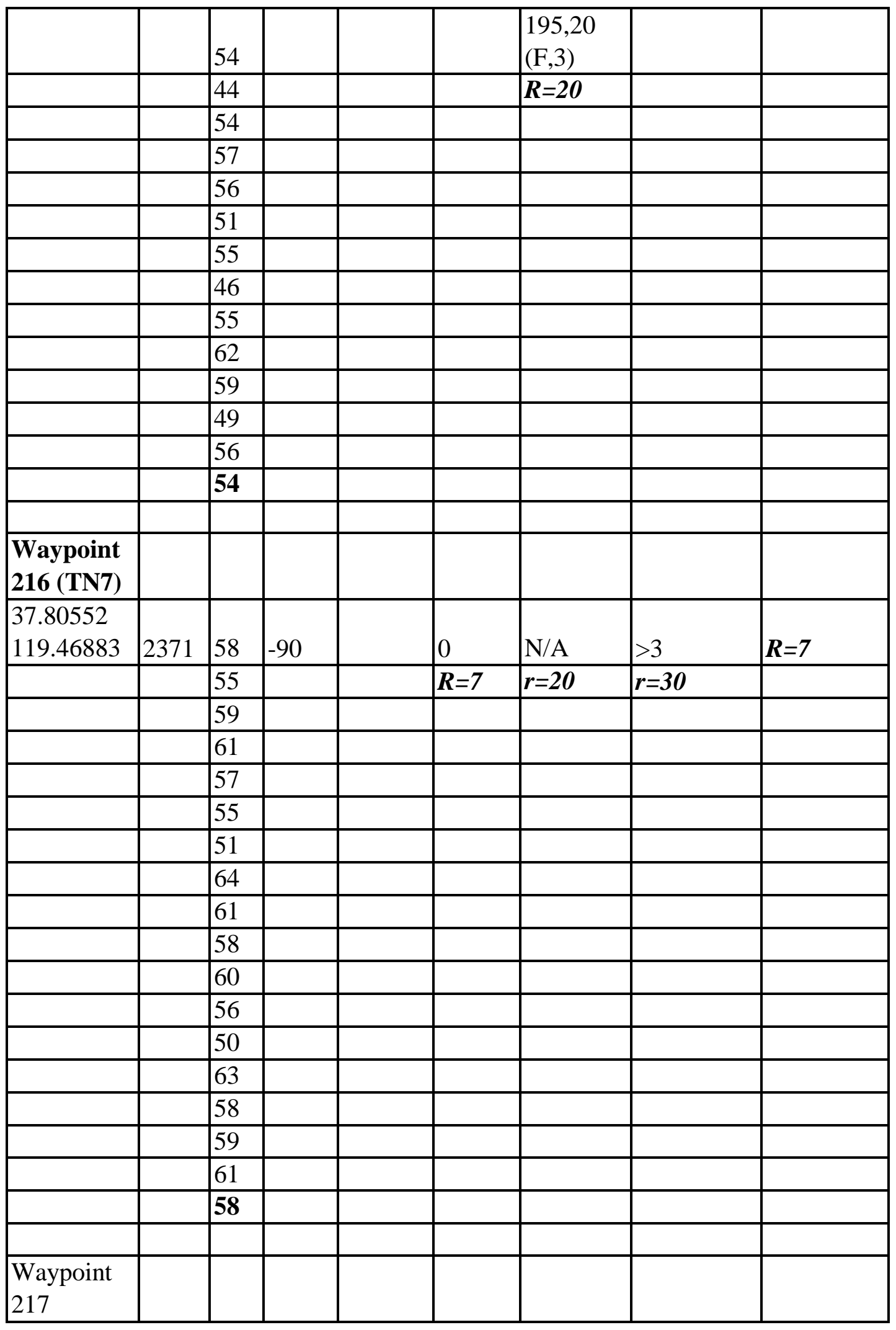




\begin{tabular}{|c|c|c|c|c|c|c|c|}
\hline $\begin{array}{l}37.80544 \\
119.46828\end{array}$ & 2384 & 64 & -90 & 5 & $\begin{array}{l}340,18 \\
(F, 2)\end{array}$ & & $R=7$ \\
\hline & & 66 & & $r=5$ & $100(\mathrm{~V})$ & $r=28$ & \\
\hline & & 65 & & & $160(\mathrm{~V})$ & & \\
\hline & & 56 & & & $r=18$ & & \\
\hline & & 58 & & & & & \\
\hline & & 57 & & & & & \\
\hline & & 59 & & & & & \\
\hline & & 56 & & & & & \\
\hline & & 58 & & & & & \\
\hline & & 57 & & & & & \\
\hline & & 65 & & & & & \\
\hline & & 62 & & & & & \\
\hline & & 63 & & & & & \\
\hline & & 58 & & & & & \\
\hline & & 58 & & & & & \\
\hline & & 58 & & & & & \\
\hline & & 59 & & & & & \\
\hline & & 60 & & & & & \\
\hline & & & & & & & \\
\hline $\begin{array}{l}\text { Waypoint } \\
218\end{array}$ & & & & & & & \\
\hline $\begin{array}{l}37.80536 \\
119.46762\end{array}$ & 2399 & 54 & -90 & 20 & 115 & 0.6 & $r=6$ \\
\hline & & 60 & & 100 & 225 & 1.8 & \\
\hline & & 61 & & 10 & 103 & 0.25 & \\
\hline & & 47 & & 30 & $r=14$ & 0.3 & \\
\hline & & 58 & & 5 & & 1.4 & \\
\hline & & 54 & & 10 & & 5.3 & \\
\hline & & 57 & & 15 & & 1 & \\
\hline & & 54 & & $r=4$ & & & \\
\hline & & 55 & & & & & \\
\hline & & 58 & & & & & \\
\hline & & 49 & & & & & \\
\hline & & 65 & & & & & \\
\hline & & 53 & & & & & \\
\hline & & 60 & & & & & \\
\hline & & 58 & & & & & \\
\hline & & 62 & & & & & \\
\hline & & 57 & & & & & \\
\hline
\end{tabular}




\begin{tabular}{|c|c|c|c|c|c|c|c|}
\hline & & & & & & & \\
\hline $\begin{array}{l}\text { Waypoint } \\
219\end{array}$ & & & & & & & \\
\hline \begin{tabular}{|l|}
37.80547 \\
119.46684
\end{tabular} & 2439 & 54 & -90 & 30 & 285,73 & 0.45 & $r=7$ \\
\hline & & 60 & & 40 & 05,80 & 0.25 & \\
\hline & & 44 & & 0.5 & 25 & 0.3 & \\
\hline & & 58 & & 1 & 285,68 & 0.4 & \\
\hline & & 58 & & 0.3 & 130,90 & 0.15 & \\
\hline & & 60 & & 20 & 100 & 0.9 & \\
\hline & & 54 & & 2 & $r=9$ & 0.35 & \\
\hline & & 63 & & 2 & & $r=15$ & \\
\hline & & 61 & & $r=5$ & & & \\
\hline & & 50 & & & & & \\
\hline & & 61 & & & & & \\
\hline & & 57 & & & & & \\
\hline & & 48 & & & & & \\
\hline & & 60 & & & & & \\
\hline & & 63 & & & & & \\
\hline & & 61 & & & & & \\
\hline & & 60 & & & & & \\
\hline & & 57 & & & & & \\
\hline & & & & & & & \\
\hline $\begin{array}{l}\text { Waypoint } \\
220 \text { (TN2) }\end{array}$ & & & & & & & \\
\hline $\begin{array}{l}37.78882 \\
119.48655 \\
\end{array}$ & 2342 & 59 & -90 & 10 & 340,80 & 1.5 & $r=5$ \\
\hline & & 61 & & 15 & $10,30(\mathrm{~F})$ & 0.9 & \\
\hline & & 58 & & 5 & 8 & 1 & \\
\hline & & 50 & & 2 & 335 & 0.45 & \\
\hline & & 53 & & 10 & 10,68 & 1.2 & \\
\hline & & 52 & & 1 & 5 & 0.45 & \\
\hline & & 55 & & 20 & 90 & 0.76 & \\
\hline & & 42 & & 1 & 350 & 1 & \\
\hline & & 53 & & 30 & 335 & 0.3 & \\
\hline & & 48 & & 1 & 350 & 0.1 & \\
\hline & & 51 & & 2 & 355 & 0.2 & \\
\hline & & 54 & & 1.5 & 185,50 & 0.1 & \\
\hline & & 48 & & 15 & 185,35 & 0.61 & \\
\hline
\end{tabular}




\begin{tabular}{|c|c|c|c|c|c|c|c|}
\hline & & 47 & & 5 & 185 & $r=21$ & \\
\hline & & 48 & & $r=4$ & $r=9$ & & \\
\hline & & 54 & & & & & \\
\hline & & 51 & & & & & \\
\hline & & 52 & & & & & \\
\hline & & & & & & & \\
\hline \multicolumn{8}{|l|}{$\begin{array}{l}\text { Waypoint } \\
221\end{array}$} \\
\hline \multirow[t]{19}{*}{$\begin{array}{l}37.78848 \\
119.48660 \\
\end{array}$} & 2347 & 64 & -90 & 30 & 355 & 0.3 & $r=6$ \\
\hline & & 61 & & 15 & 255 & 1 & \\
\hline & & 53 & & 10 & 180,68 & 0.2 & \\
\hline & & 52 & & 10 & $\begin{array}{l}280,15 \\
(F, 4)\end{array}$ & 0.6 & \\
\hline & & 61 & & 35 & 270,75 & 0.3 & \\
\hline & & 51 & & 5 & $r=9$ & 0.9 & \\
\hline & & 59 & & 15 & & 1.2 & \\
\hline & & 61 & & 25 & & 0.6 & \\
\hline & & 60 & & 20 & & $r=21$ & \\
\hline & & 63 & & 15 & & & \\
\hline & & 54 & & $r=4$ & & & \\
\hline & & 60 & & & & & \\
\hline & & 51 & & & & & \\
\hline & & 59 & & & & & \\
\hline & & 55 & & & & & \\
\hline & & 65 & & & & & \\
\hline & & 63 & & & & & \\
\hline & & 58 & & & & & \\
\hline & & & & & & & \\
\hline \multicolumn{8}{|l|}{$\begin{array}{l}\text { Waypoint } \\
222\end{array}$} \\
\hline $\begin{array}{l}37.78806 \\
119.48665\end{array}$ & 2333 & 60 & -90 & 0 & N/A & $>3$ & 7 \\
\hline & & 60 & & $r=7$ & $r=20$ & $r=30$ & \\
\hline & & 60 & & & & & \\
\hline & & 56 & & & & & \\
\hline & & 53 & & & & & \\
\hline & & 64 & & & & & \\
\hline & & 60 & & & & & \\
\hline & & 55 & & & & & \\
\hline
\end{tabular}




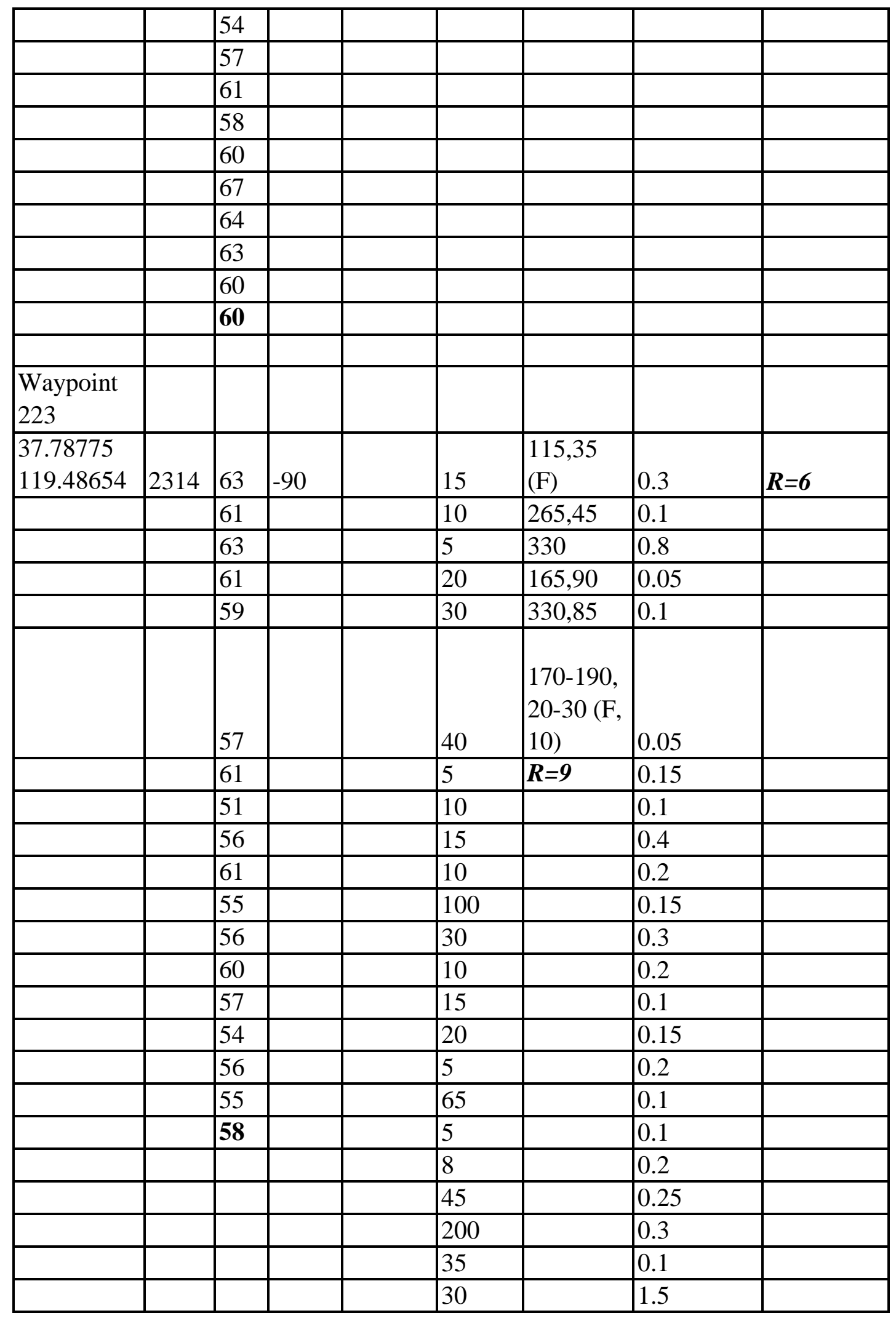




\begin{tabular}{|c|c|c|c|c|c|c|c|}
\hline & & & & 15 & & 0.15 & \\
\hline & & & & $R=4$ & & $R=15$ & \\
\hline & & & & & & & \\
\hline \multicolumn{8}{|l|}{$\begin{array}{l}\text { Waypoint } \\
225\end{array}$} \\
\hline \multirow[t]{18}{*}{\begin{tabular}{|l|}
37.78715 \\
119.48634 \\
\end{tabular}} & 2291 & 57 & -90 & 0 & N/A & $>3$ & \\
\hline & & 51 & & $r=7$ & $r=20$ & $r=30$ & $r=7$ \\
\hline & & 53 & & & & & \\
\hline & & 50 & & & & & \\
\hline & & 54 & & & & & \\
\hline & & 59 & & & & & \\
\hline & & 55 & & & & & \\
\hline & & 54 & & & & & \\
\hline & & 57 & & & & & \\
\hline & & 55 & & & & & \\
\hline & & 58 & & & & & \\
\hline & & 52 & & & & & \\
\hline & & 56 & & & & & \\
\hline & & 60 & & & & & \\
\hline & & 53 & & & & & \\
\hline & & 48 & & & & & \\
\hline & & 55 & & & & & \\
\hline & & 55 & & & & & \\
\hline & & & & & & & \\
\hline \multicolumn{8}{|l|}{$\begin{array}{l}\text { Waypoint } \\
226 \text { (TN3) }\end{array}$} \\
\hline \begin{tabular}{|l|}
37.78688 \\
119.48624
\end{tabular} & 2302 & 65 & -90 & 3 & $\begin{array}{l}95,20(\mathrm{~F}, \\
12)\end{array}$ & 0.9 & $r=4$ \\
\hline & & 58 & & 5 & $0,10(\mathrm{~F})$ & 1.8 & \\
\hline & & 61 & & 10 & 235,80 & 1.4 & \\
\hline & & 64 & & 8 & 120 & 0.3 & \\
\hline & & 57 & & 15 & 55 & 0.25 & \\
\hline & & 65 & & 10 & $r=9$ & 0.5 & \\
\hline & & 64 & & 20 & & 0.3 & \\
\hline & & 56 & & 5 & & 0.1 & \\
\hline & & 66 & & 20 & & 0.4 & \\
\hline & & 58 & & 2 & & $r=21$ & \\
\hline & & 65 & & 1 & & & \\
\hline & & 57 & & 15 & & & \\
\hline
\end{tabular}




\begin{tabular}{|c|c|c|c|c|c|c|c|}
\hline & & 60 & & 30 & & & \\
\hline & & 60 & & 60 & & & \\
\hline & & 60 & & 10 & & & \\
\hline & & 62 & & $r=4$ & & & \\
\hline & & 63 & & & & & \\
\hline & & 61 & & & & & \\
\hline & & & & & & & \\
\hline \multicolumn{8}{|l|}{$\begin{array}{l}\text { Waypoint } \\
227\end{array}$} \\
\hline \begin{tabular}{|l|}
37.78662 \\
119.48627
\end{tabular} & 2314 & 60 & -45 & 20 & $\begin{array}{l}75,40 \\
(F, 8)\end{array}$ & 1 & $r=5$ \\
\hline & & 48 & & 15 & 225,80 & 0.6 & \\
\hline & & 60 & & 10 & 185,90 & 0.4 & \\
\hline & & 52 & & 30 & 250,86 & 0.2 & \\
\hline & & 58 & & 10 & 95,20 & 0.3 & \\
\hline & & 58 & & 35 & 160 & 0.25 & \\
\hline & & 52 & & 15 & 265,15 & 0.6 & \\
\hline & & 60 & & 10 & $r=9$ & 0.1 & \\
\hline & & 54 & & 5 & & 0.9 & \\
\hline & & 55 & & 15 & & 0.05 & \\
\hline & & 60 & & $r=4$ & & 0.35 & \\
\hline & & 58 & & & & $r=21$ & \\
\hline & & 55 & & & & & \\
\hline & & 55 & & & & & \\
\hline & & 61 & & & & & \\
\hline & & 59 & & & & & \\
\hline & & 61 & & & & & \\
\hline & & 57 & & & & & \\
\hline & & & & & & & \\
\hline $\begin{array}{l}\text { Waypoint } \\
228\end{array}$ & & & & & & & \\
\hline $\begin{array}{l}37.78614 \\
119.48608\end{array}$ & 2346 & 68 & -90 & 15 & 175,80 & 0.3 & $r=6$ \\
\hline & & 65 & & 5 & 120,84 & 1.2 & \\
\hline & & 66 & & 20 & 165,90 & 0.45 & \\
\hline & & 59 & & 15 & 45 & 0.2 & \\
\hline & & 63 & & 10 & 115 & 0.1 & \\
\hline & & 66 & & 20 & 135,40 & 0.2 & \\
\hline & & 64 & & 5 & 315,55 & 0.35 & \\
\hline & & 64 & & 20 & 345,5 & 0.5 & \\
\hline
\end{tabular}




\begin{tabular}{|c|c|c|c|c|c|c|c|}
\hline & & 65 & & 15 & 95 & 0.15 & \\
\hline & & 64 & & 120 & 285 & 0.1 & \\
\hline & & 67 & & 40 & 325,80 & 0.2 & \\
\hline & & 64 & & 30 & 320,20 & 0.1 & \\
\hline & & 65 & & 17.5 & 290 & 0.9 & \\
\hline & & 64 & & $r=4$ & $115,8(\mathrm{~F})$ & 0.6 & \\
\hline & & 63 & & & 120,86 & 0.15 & \\
\hline & & 67 & & & $\begin{array}{l}\text { Flakes } \\
\text { striking } \\
\text { roughly } \\
\text { east/NE }\end{array}$ & 0.5 & \\
\hline & & 68 & & & $r=9$ & 1 & \\
\hline & & 65 & & & & 0.2 & \\
\hline & & & & & & 0.15 & \\
\hline & & & & & & 0.6 & \\
\hline & & & & & & 0.25 & \\
\hline & & & & & & $r=15$ & \\
\hline & & & & & & & \\
\hline $\begin{array}{l}\text { Waypoint } \\
229\end{array}$ & & & & & & & \\
\hline $\begin{array}{l}37.78581 \\
119.48599\end{array}$ & 2364 & 65 & -90 & 130 & 270,60 & 0.6 & $r=6$ \\
\hline & & 67 & & 140 & 175,86 & 1.2 & \\
\hline & & 68 & & 30 & 315,80 & 1.2 & \\
\hline & & 70 & & 20 & 350 & 0.6 & \\
\hline & & 66 & & 110 & 280 & 0.9 & \\
\hline & & 55 & & 10 & 180,75 & 1.5 & \\
\hline & & 58 & & 25 & $r=14$ & 0.2 & \\
\hline & & 60 & & 40 & & 1.4 & \\
\hline & & 69 & & 35 & & 1.05 & \\
\hline & & 66 & & $r=2$ & & $r=28$ & \\
\hline & & 58 & & & & & \\
\hline & & 65 & & & & & \\
\hline & & 67 & & & & & \\
\hline & & 58 & & & & & \\
\hline & & 68 & & & & & \\
\hline & & 57 & & & & & \\
\hline & & 68 & & & & & \\
\hline & & 64 & & & & & \\
\hline
\end{tabular}




\begin{tabular}{|c|c|c|c|c|c|c|c|}
\hline \multirow{2}{*}{$\begin{array}{l}\begin{array}{l}\text { Waypoint } \\
\text { 230 } \text { (TN1) }\end{array} \\
37.78648 \\
119.48923 \\
\end{array}$} & & & & & & & \\
\hline & 2266 & 64 & -45 & 60 & 280,76 & 1 & $r=6$ \\
\hline & & 65 & & 15 & \begin{tabular}{|l|}
195,5 \\
\end{tabular} & 0.6 & \\
\hline & & 65 & & 30 & 265 & 1.2 & \\
\hline & & 61 & & 80 & 260,75 & 0.6 & \\
\hline & & 69 & & 20 & $r=9$ & 1.5 & \\
\hline & & 71 & & 75 & & 0.5 & \\
\hline & & 66 & & 25 & & 0.8 & \\
\hline & & 58 & & 10 & & $r=21$ & \\
\hline & & 65 & & 27.5 & & & \\
\hline & & 57 & & $r=2$ & & & \\
\hline & & 67 & & & & & \\
\hline & & 69 & & & & & \\
\hline & & 66 & & & & & \\
\hline & & 65 & & & & & \\
\hline & & 56 & & & & & \\
\hline & & 69 & & & & & \\
\hline & & 67 & & & & & \\
\hline & & 65 & & & & & \\
\hline & & & & & & & \\
\hline $\begin{array}{l}\text { Waypoint } \\
231\end{array}$ & & & & & & & \\
\hline $\begin{array}{l}37.78637 \\
119.48890\end{array}$ & 2281 & 60 & -90 & 10 & 270,84 & 0.6 & $r=7$ \\
\hline & & 60 & & 15 & 105 & 0.3 & \\
\hline & & 48 & & 5 & 115,70 & 0.6 & \\
\hline & & 57 & & 6 & 95 & 1 & \\
\hline & & 50 & & 5 & 70,80 & 0.9 & \\
\hline & & 54 & & 2 & $\begin{array}{l}160,10 \\
(\mathrm{~F})\end{array}$ & 0.4 & \\
\hline & & 53 & & 20 & $15,25(\mathrm{~F})$ & 0.9 & \\
\hline & & 54 & & 1 & 20,76 & 0.6 & \\
\hline & & 53 & & 3 & 275,68 & $r=21$ & \\
\hline & & 50 & & 5 & $r=18$ & & \\
\hline & & 56 & & $r=4$ & & & \\
\hline & & 64 & & & & & \\
\hline
\end{tabular}




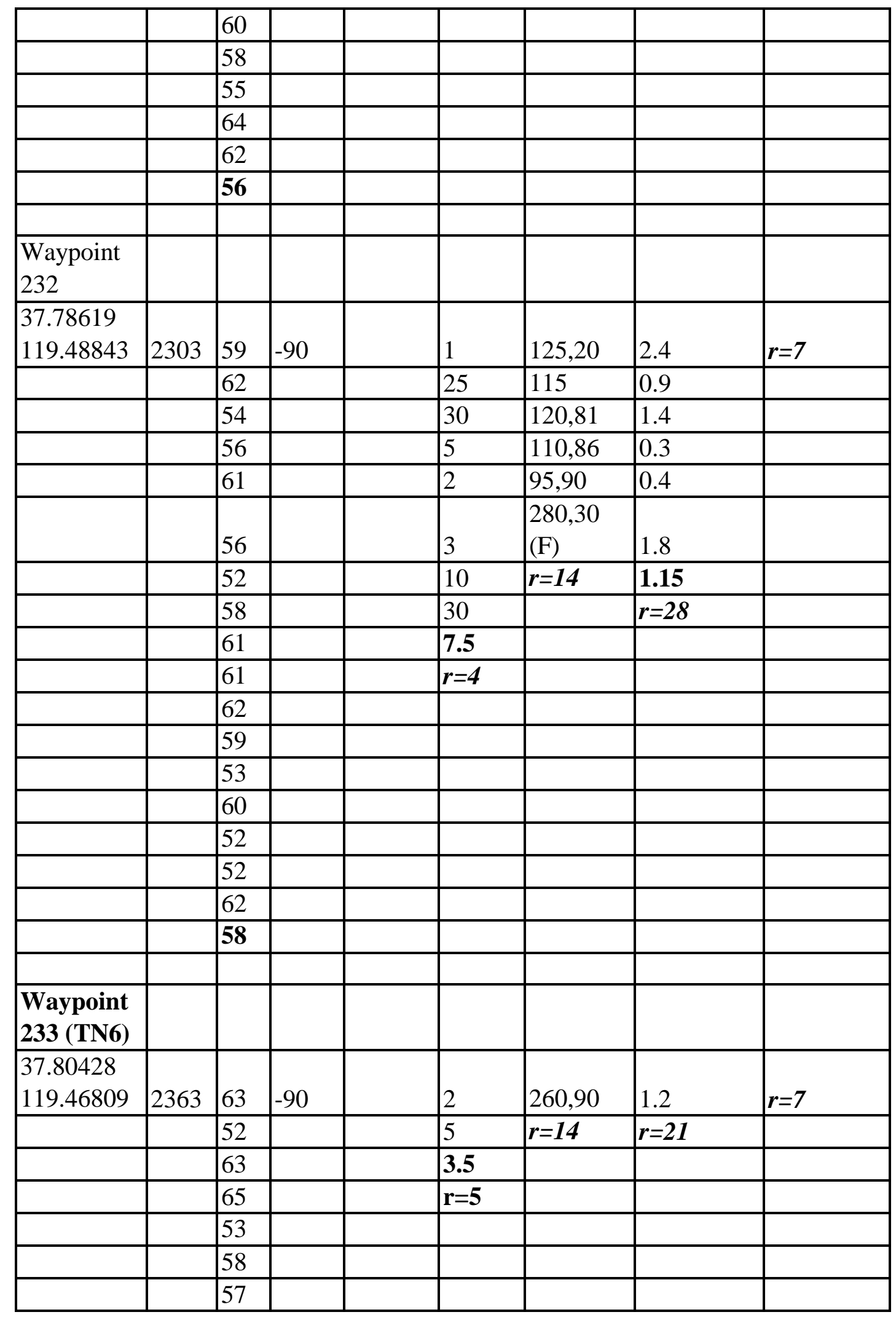




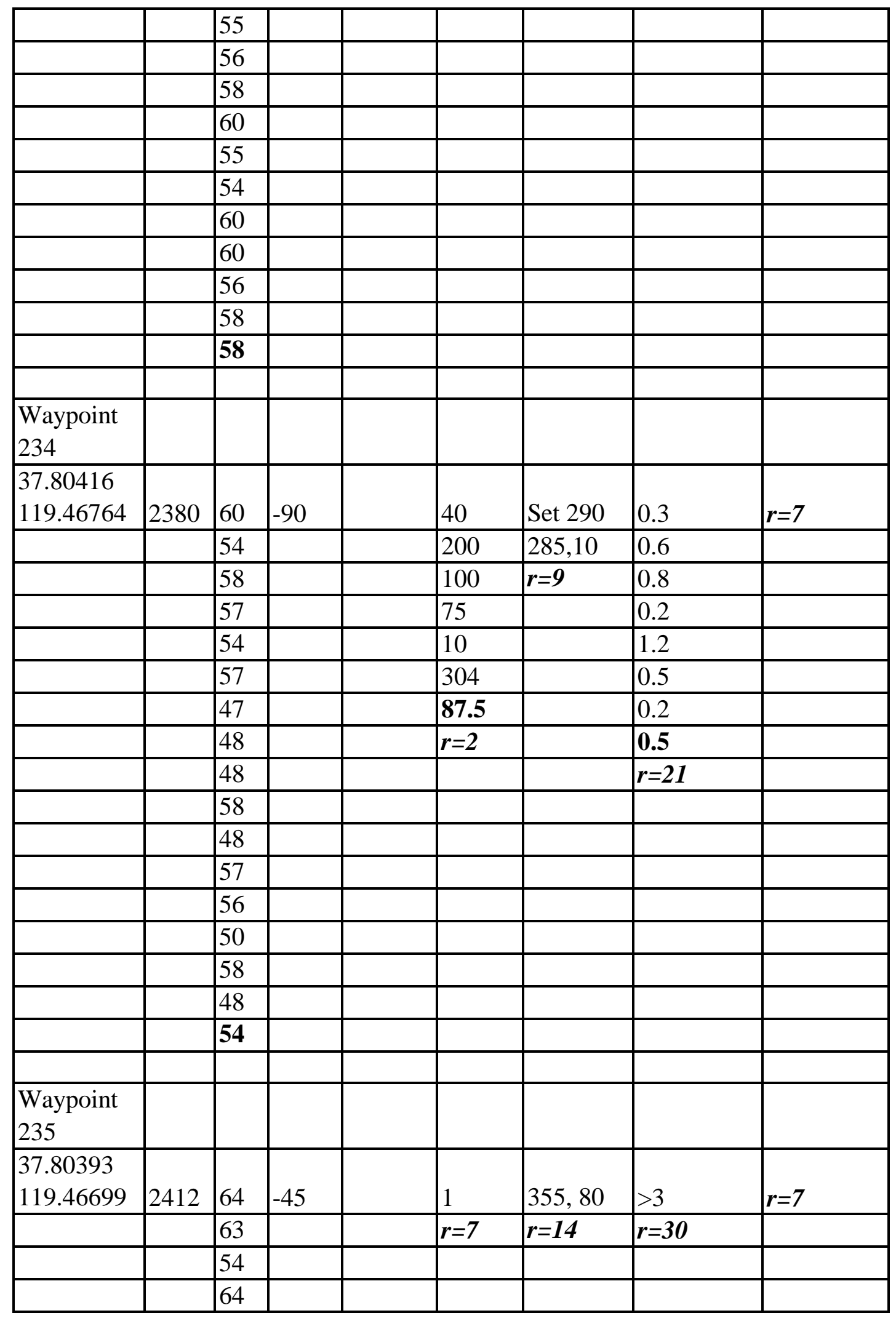




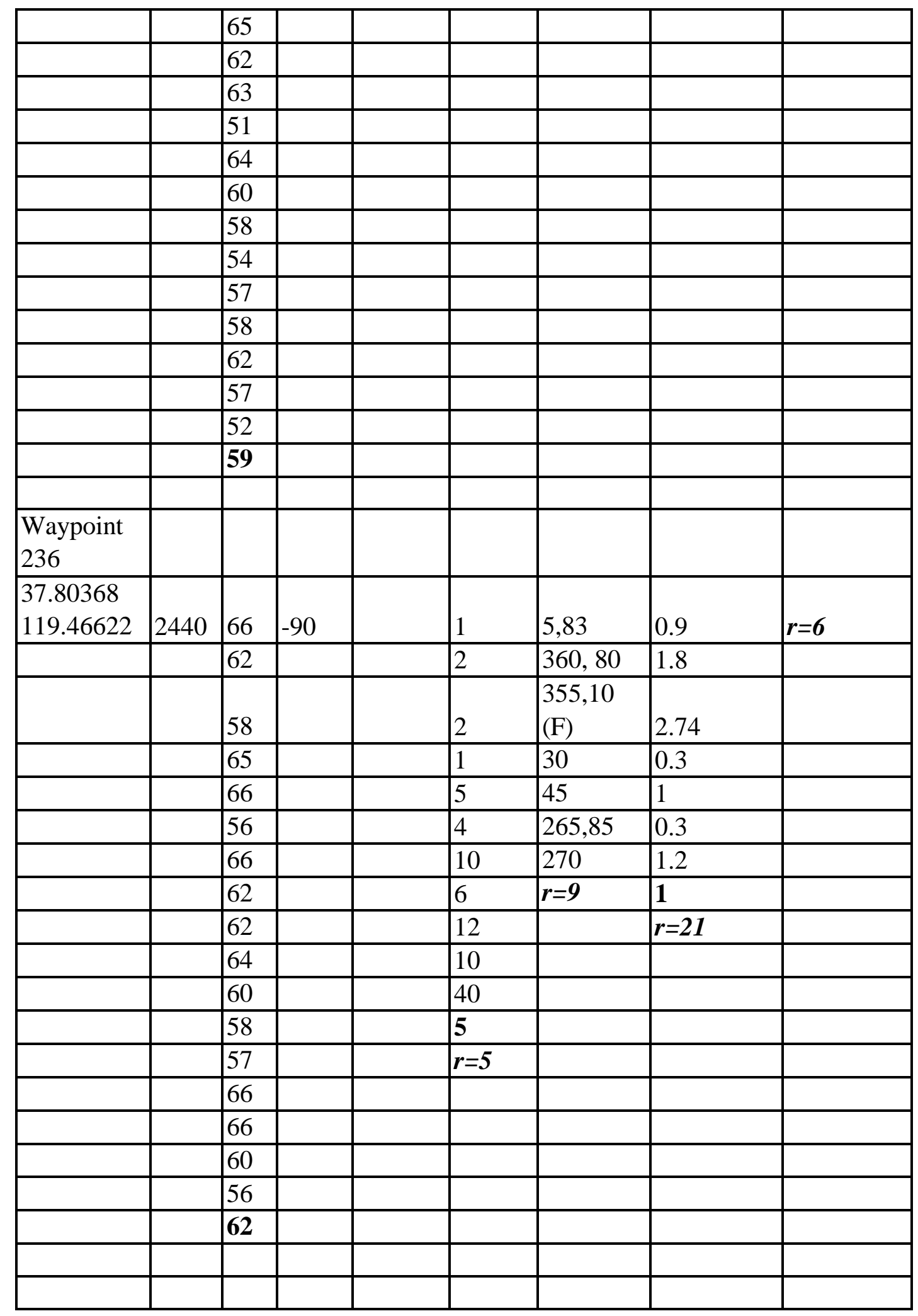




\begin{tabular}{|c|c|c|c|c|c|c|c|}
\hline $\begin{array}{l}\text { Waypoint } \\
237\end{array}$ & & & & & & & \\
\hline $\begin{array}{l}37.80450 \\
119.46854\end{array}$ & 2359 & 60 & -90 & 0 & 150 & $>3$ & $r=7$ \\
\hline & & 58 & & $r=7$ & $r=20$ & $r=30$ & \\
\hline & & 58 & & & & & \\
\hline & & 64 & & & & & \\
\hline & & 60 & & & & & \\
\hline & & 64 & & & & & \\
\hline & & 60 & & & & & \\
\hline & & 57 & & & & & \\
\hline & & 61 & & & & & \\
\hline & & 50 & & & & & \\
\hline & & 48 & & & & & \\
\hline & & 52 & & & & & \\
\hline & & 60 & & & & & \\
\hline & & 60 & & & & & \\
\hline & & 55 & & & & & \\
\hline & & 58 & & & & & \\
\hline & & 58 & & & & & \\
\hline & & 58 & & & & & \\
\hline & & & & & & & \\
\hline $\begin{array}{l}\text { Waypoint } \\
238\end{array}$ & & & & & & & \\
\hline $\begin{array}{l}37.80460 \\
119.46944\end{array}$ & 2355 & 60 & -90 & $r=7$ & $r=20$ & $r=30$ & $r=7$ \\
\hline & & 54 & & & & & \\
\hline & & 50 & & & & & \\
\hline & & 54 & & & & & \\
\hline & & 60 & & & & & \\
\hline & & 60 & & & & & \\
\hline & & 65 & & & & & \\
\hline & & 59 & & & & & \\
\hline & & 62 & & & & & \\
\hline & & 58 & & & & & \\
\hline & & 61 & & & & & \\
\hline & & 54 & & & & & \\
\hline & & 60 & & & & & \\
\hline & & 57 & & & & & \\
\hline & & 60 & & & & & \\
\hline
\end{tabular}




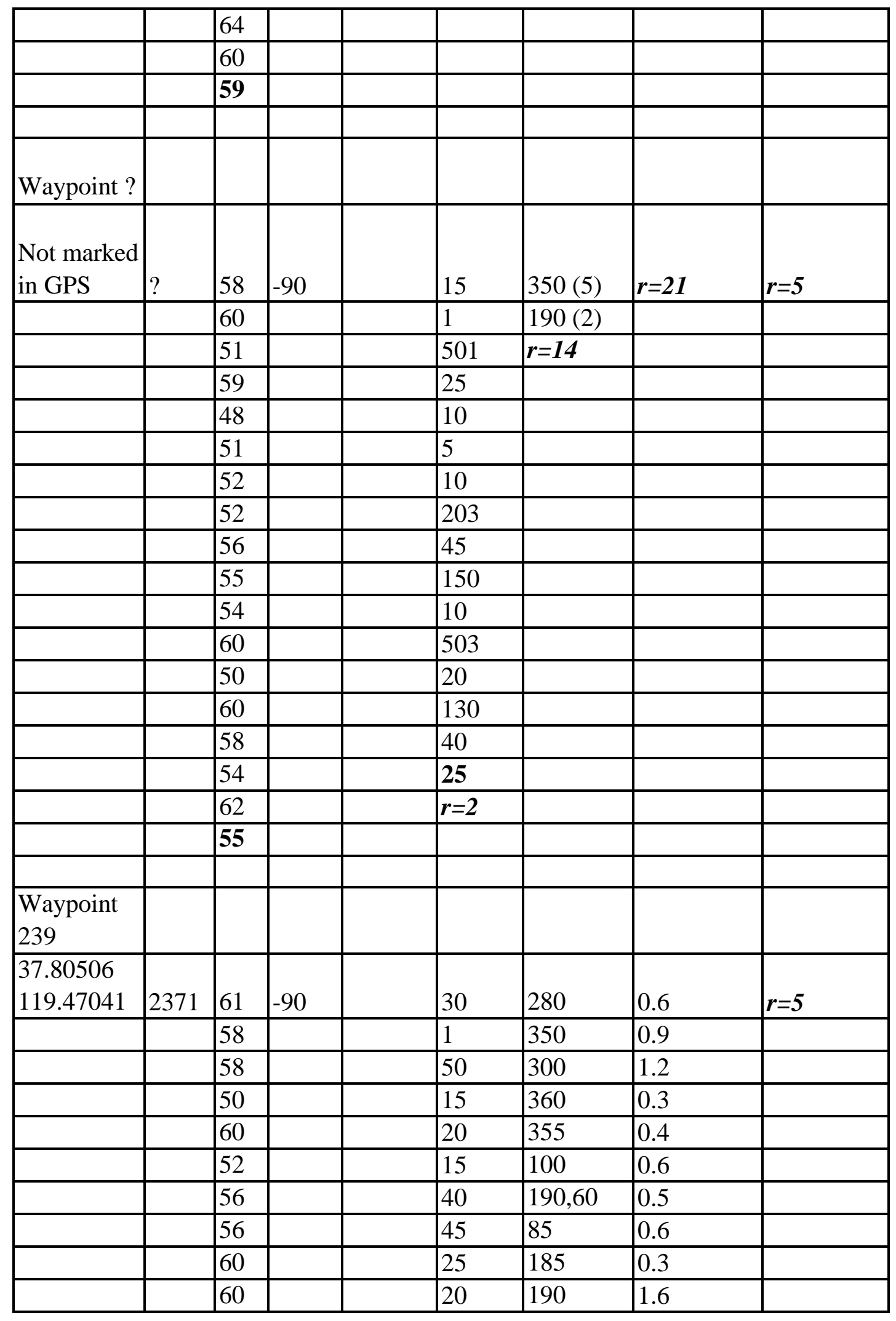




\begin{tabular}{|c|c|c|c|c|c|c|c|}
\hline & & 60 & & 35 & 95 & 1 & \\
\hline & & 50 & & 30 & 70 & 0.4 & \\
\hline & & 61 & & 60 & 175,90 & 0.5 & \\
\hline & & 54 & & 50 & $r=9$ & 0.4 & \\
\hline & & 54 & & 10 & & 0.3 & \\
\hline & & 61 & & 2 & & 0.2 & \\
\hline & & 60 & & 27.5 & & 0.5 & \\
\hline & & 57 & & $r=2$ & & $r=21$ & \\
\hline & & & & & & & \\
\hline \multicolumn{8}{|l|}{$\begin{array}{l}\text { Waypoint } \\
240\end{array}$} \\
\hline \multirow[t]{18}{*}{$\begin{array}{l}37.80532 \\
119.47142 \\
\end{array}$} & 2390 & 64 & -90 & 40 & 290 & 1.5 & $r=6$ \\
\hline & & 63 & & 20 & $265(\mathrm{~F})$ & 1 & \\
\hline & & 63 & & 15 & $r=14$ & 2.7 & \\
\hline & & 64 & & 20 & & 0.2 & \\
\hline & & 68 & & $r=4$ & & 0.3 & \\
\hline & & 66 & & & & 1 & \\
\hline & & 67 & & & & $r=28$ & \\
\hline & & 66 & & & & & \\
\hline & & 68 & & & & & \\
\hline & & 63 & & & & & \\
\hline & & 68 & & & & & \\
\hline & & 65 & & & & & \\
\hline & & 67 & & & & & \\
\hline & & 70 & & & & & \\
\hline & & 69 & & & & & \\
\hline & & 68 & & & & & \\
\hline & & 66 & & & & & \\
\hline & & 66 & & & & & \\
\hline & & & & & & & \\
\hline \multicolumn{8}{|l|}{$\begin{array}{l}\text { Waypoint } \\
241 \text { (TN4) }\end{array}$} \\
\hline $\begin{array}{l}37.80061 \\
119.47329\end{array}$ & 2341 & 55 & -90 & $r=7$ & $r=20$ & $r=30$ & $r=7$ \\
\hline & & 54 & & & & & \\
\hline & & 56 & & & & & \\
\hline & & 66 & & & & & \\
\hline & & 57 & & & & & \\
\hline & & 56 & & & & & \\
\hline
\end{tabular}




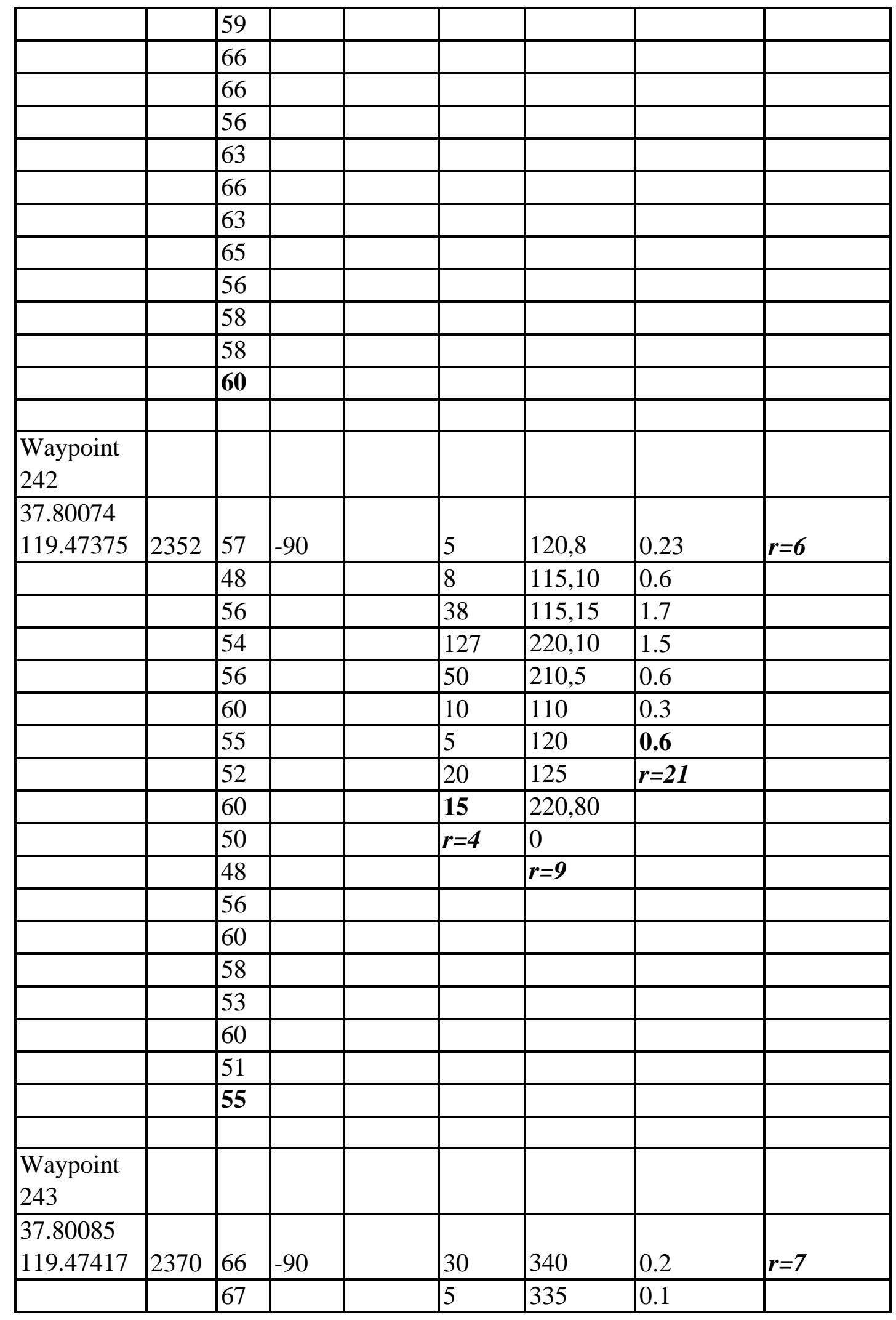




\begin{tabular}{|l|l|l|l|l|l|l|l|l|}
\hline & & 64 & & & 3 & 340 & 0.3 & \\
\hline & & 62 & & & 1 & 200 & 0.9 & \\
\hline & & 62 & & & 5 & 330 & 0.5 & \\
\hline & & 60 & & & 50 & 190,32 & 0.9 & \\
\hline & & 65 & & & 25 & 195 & 0.6 & \\
\hline & & 62 & & & 80 & 165 & 0.2 & \\
\hline & & 61 & & & 10 & 280 & 0.1 & \\
\hline & & 64 & & & 10 & 185 & 0.4 & \\
\hline & & 62 & & & 5 & 180 & 0.3 & \\
\hline & & 57 & & & 100 & 145 & 0.6 & \\
\hline & & 60 & & & 2 & 140 & 0.9 & \\
\hline & & 58 & & & 40 & $\boldsymbol{r}=\mathbf{9}$ & 0.3 & \\
\hline & & 66 & & & 5 & & 0.25 & \\
\hline & & 55 & & & $\mathbf{1 0}$ & & 0.15 & \\
\hline & & 64 & & & $\boldsymbol{r}=\mathbf{4}$ & & 0.15 & \\
\hline & & $\mathbf{6 2}$ & & & & & $\mathbf{0 . 3}$ & \\
\hline & & & & & & & $\boldsymbol{r}=\mathbf{1 5}$ & \\
\hline
\end{tabular}




\begin{tabular}{|c|c|c|c|c|c|c|c|c|}
\hline \multicolumn{9}{|c|}{ Little Yosemite Valley } \\
\hline \multicolumn{9}{|c|}{\begin{tabular}{|l|l|l|} 
Waypoint & & \\
166 (LY7) & & \\
\end{tabular}} \\
\hline Lat/Long & $\begin{array}{l}\text { Elev. } \\
(\mathrm{m})\end{array}$ & $\mathrm{SH}$ & Alpha & \begin{tabular}{|l} 
Joint \\
length \\
(m)
\end{tabular} & $\begin{array}{l}\text { Joint } \\
\text { width } \\
(\mathrm{mm}) \\
\end{array}$ & \begin{tabular}{|l} 
Joint \\
strike and \\
dip
\end{tabular} & $\begin{array}{l}\text { Joint } \\
\text { spacing } \\
\text { (m) }\end{array}$ & Continuity \\
\hline \multirow[t]{19}{*}{\begin{tabular}{|l|}
37.73994 \\
119.42727 \\
\end{tabular}} & 2170 & 66 & -90 & $>5(1)$ & na & 220 & 0.3 & $r=6$ \\
\hline & & 48 & & 3-4.5 (2) & 0.1 & 170 & 1.2 & \\
\hline & & 68 & & $2(3)$ & 0.1 & 170 & 1.5 & \\
\hline & & 72 & & $2.5(4)$ & na & 210 & 0.6 & \\
\hline & & 65 & & $2(5)$ & 0.1 & 181 & 0.9 & \\
\hline & & 66 & & $\begin{array}{l}>3,>15 \\
(6)\end{array}$ & na & 215 & 0.6 & \\
\hline & & 66 & & & $r=7$ & $r=20$ & 2 & \\
\hline & & 72 & & & & & 4 & \\
\hline & & 72 & & & & & 0.9 & \\
\hline & & 66 & & & & & 1.33 & \\
\hline & & 72 & & & & & 0.9 & \\
\hline & & 72 & & & & & $r=21$ & \\
\hline & & 71 & & & & & & \\
\hline & & 64 & & & & & & \\
\hline & & 68 & & & & & & \\
\hline & & 70 & & & & & & \\
\hline & & 69 & & & & & & \\
\hline & & 67 & & & & & & \\
\hline & & & & & & & & \\
\hline \multicolumn{9}{|l|}{$\begin{array}{l}\text { Waypoint } \\
167\end{array}$} \\
\hline $\begin{array}{l}37.73887 \\
119.42755\end{array}$ & 2202 & 51 & -90 & $>20(1)$ & 0.5 & 200 & 1.2 & $r=6$ \\
\hline & & 48 & & $4(2)$ & 1 & 170 & 0.6 & \\
\hline & & 62 & & $3.5(3)$ & 5 & 220 & 0.3 & \\
\hline & & 55 & & $3.5(4)$ & na & 215 & 5 & \\
\hline & & 47 & & $4(5)$ & na & 170 & 0.2 & \\
\hline
\end{tabular}




\begin{tabular}{|c|c|c|c|c|c|c|c|c|}
\hline & & 52 & & $\begin{array}{l}>10(\mathrm{~V} 1) \\
\text { Very } \\
\text { fractured }\end{array}$ & 15 & 120 & 4 & \\
\hline & & 57 & & $>6$ (V2) & 10 & 150 & $\mid 0.9$ & \\
\hline & & 55 & & $0.9(6)$ & $r=4$ & $r=14$ & $r=21$ & \\
\hline & & 54 & & & & & & \\
\hline & & 61 & & & & & & \\
\hline & & 63 & & & & & & \\
\hline & & 62 & & & & & & \\
\hline & & 56 & & & & & & \\
\hline & & 51 & & & & & & \\
\hline & & 56 & & & & & & \\
\hline & & 54 & & & & & & \\
\hline & & 60 & & & & & & \\
\hline & & 56 & & & & & & \\
\hline & & & & & & & & \\
\hline $\begin{array}{l}\text { Waypoint } \\
168 \text { (LY8) }\end{array}$ & & & & & & & & \\
\hline $\begin{array}{l}37.72949 \\
119.39222\end{array}$ & 2238 & 54 & -90 & $3.5(1)$ & 5 & 55 & 1.2 & $r=6$ \\
\hline & & 50 & & $10(2)$ & 75 & 345,90 & 0.3 & \\
\hline & & 52 & & $1.5(3)$ & 2 & 110 & 0.6 & \\
\hline & & 53 & & $1.5(\mathrm{~F} 1)$ & 150 & 325 & 0.3 & \\
\hline & & 48 & & $1(\mathrm{~F} 2)$ & 5 & 35 & 0.1 & \\
\hline & & 54 & & & 5 & $r=9$ & 0.2 & \\
\hline & & 52 & & & $r=4$ & & 0.5 & \\
\hline & & 50 & & & & & 0.2 & \\
\hline & & 56 & & & & & 0.4 & \\
\hline & & 57 & & & & & 0.6 & \\
\hline & & 52 & & & & & 1.5 & \\
\hline & & 54 & & & & & \begin{tabular}{|l|}
0.4 \\
\end{tabular} & \\
\hline & & 52 & & & & & $r=21$ & \\
\hline & & 53 & & & & & & \\
\hline & & 50 & & & & & & \\
\hline & & 52 & & & & & & \\
\hline & & 52 & & & & & & \\
\hline & & & & & & & & \\
\hline
\end{tabular}




\begin{tabular}{|c|c|c|c|c|c|c|c|c|}
\hline \multicolumn{9}{|l|}{$\begin{array}{l}\text { Waypoint } \\
169\end{array}$} \\
\hline $\begin{array}{l}37.72964 \\
119.39174\end{array}$ & 2263 & 56 & -45 & $>20(1)$ & na & 60 & 2 & $r=6$ \\
\hline & & 56 & & $>5(2)$ & 0.2 & 55 & 1.2 & \\
\hline & & 58 & & $2(3)$ & 1 & 10 & 3 & \\
\hline & & $\overline{53}$ & & $>10(4)$ & 0.1 & 60 & 1.5 & \\
\hline & & 60 & & $>15(\mathrm{~V} 1)$ & 0.1 & 135 & 2 & \\
\hline & & $\overline{58}$ & & 10 (V2) & 0.3 & 130 & 2.5 & \\
\hline & & 57 & & $>10(\mathrm{~V} 3)$ & na & 135 & 2.03 & \\
\hline & & 55 & & & $r=7$ & $r=20$ & 2 & \\
\hline & & 48 & & & & & $r=28$ & \\
\hline & & 62 & & & & & & \\
\hline & & 62 & & & & & & \\
\hline & & 56 & & & & & & \\
\hline & & 54 & & & & & & \\
\hline & & 56 & & & & & & \\
\hline & & 52 & & & & & & \\
\hline & & 53 & & & & & & \\
\hline & & 64 & & & & & & \\
\hline & & 56 & & & & & & \\
\hline & & & & & & & & \\
\hline $\begin{array}{l}\text { Waypoint } \\
170\end{array}$ & 2279 & 50 & -90 & $2(1)$ & 1 & 60 & 3 & $r=5$ \\
\hline $\begin{array}{l}37.72975 \\
119.39133\end{array}$ & & 58 & & $4(2)$ & 130 & 65,90 & 0.3 & \\
\hline & & 48 & & $2(3)$ & 10 & 45,90 & 1.2 & \\
\hline & & 61 & & 1 (4) & 20 & $160, .90$ & 2 & \\
\hline & & 57 & & $4(5)$ & 5 & 90,84 & 0.9 & \\
\hline & & 51 & & \begin{tabular}{|l|l}
$6(\mathrm{~F} 1)$ \\
\end{tabular} & 130 & 70 & 2 & \\
\hline & & 61 & & $2(\mathrm{~F} 2)$ & 5 & \begin{tabular}{|l|}
175,30 \\
\end{tabular} & 1 & \\
\hline & & 66 & & 1 (F3) & 1 & 165,50 & 0.2 & \\
\hline & & 64 & & $2.5(\mathrm{~F} 4)$ & na & 225,60 & 0.9 & \\
\hline & & 56 & & & 7.5 & $r=9$ & 1 & \\
\hline & & 52 & & & $r=4$ & & $r=28$ & \\
\hline & & 58 & & & & & & \\
\hline & & 53 & & & & & & \\
\hline & & 60 & & & & & & \\
\hline
\end{tabular}




\begin{tabular}{|l|l|l|l|l|l|l|l|l|}
\hline & & 52 & & & & & & \\
\hline & & 55 & & & & & & \\
\hline & & 53 & & & & & & \\
\hline & & $\mathbf{5 7}$ & & & & & & \\
\hline & & & & & & & & \\
\hline $\begin{array}{l}\text { Waypoint } \\
171\end{array}$ & & & & & & & & \\
\hline 37.73012 \\
119.39083
\end{tabular}




\begin{tabular}{|c|c|c|c|c|c|c|c|c|}
\hline & & 48 & & & & & & \\
\hline & & 52 & & & & & & \\
\hline & & 47 & & & & & & \\
\hline & & 50 & & & & & & \\
\hline & & 51 & & & & & & \\
\hline & & 53 & & & & & & \\
\hline & & 54 & & & & & & \\
\hline & & 51 & & & & & & \\
\hline & & 56 & & & & & & \\
\hline & & 60 & & & & & & \\
\hline & & 58 & & & & & & \\
\hline & & 53 & & & & & & \\
\hline & & & & & & & & \\
\hline $\begin{array}{l}\text { Waypoint } \\
173\end{array}$ & & & & & & & & \\
\hline $\begin{array}{l}37.74169 \\
119.44644\end{array}$ & 2151 & 54 & -45 & $2(1)$ & 2.5 & 150,85 & 0.3 & $r=6$ \\
\hline & & 54 & & 1 (Set 1$)$ & 20 & 225,80 & 0.2 & \\
\hline & & 57 & & $2.5(\mathrm{~F} 1)$ & 5 & 10 & 0.2 & \\
\hline & & 58 & & $3(2)$ & 20 & 310 & 0.3 & \\
\hline & & 62 & & $>5(\mathrm{~V} 1)$ & 10 & 355 & 0.5 & \\
\hline & & 58 & & $>4(\mathrm{~V} 2)$ & 30.5 & 355 & 4 & \\
\hline & & 60 & & $>4(\mathrm{~V} 3)$ & 122 & 0 & 3.5 & \\
\hline & & 56 & & & $r=4$ & $r=9$ & 2.5 & \\
\hline & & 62 & & & & & 2 & \\
\hline & & 64 & & & & & 3 & \\
\hline & & 52 & & & & & 1.65 & \\
\hline & & 54 & & & & & 1.25 & \\
\hline & & 58 & & & & & $r=28$ & \\
\hline & & 62 & & & & & & \\
\hline & & 56 & & & & & & \\
\hline & & 62 & & & & & & \\
\hline & & 62 & & & & & & \\
\hline & & 58 & & & & & & \\
\hline & & & & & & & & \\
\hline $\begin{array}{l}\text { Waypoint } \\
174\end{array}$ & & & & & & & & \\
\hline $\begin{array}{l}37.73779 \\
119.45259\end{array}$ & & & & & & & & \\
\hline
\end{tabular}




\begin{tabular}{|c|c|c|c|c|c|c|c|c|}
\hline \multicolumn{9}{|l|}{$\begin{array}{l}\text { Waypoint } \\
175 \text { (LY5) }\end{array}$} \\
\hline $\begin{array}{l}37.74176 \\
119.45863\end{array}$ & 2032 & 55 & -90 & $>9,4(1)$ & 1 & 30,86 & 0.3 & $r=6$ \\
\hline & & 55 & & $0.5-2(2)$ & 17.5 & 65 & 0.9 & \\
\hline & & 47 & & $1.75(3)$ & 14 & 85 & 2 & \\
\hline & & 60 & & $>9(4)$ & 5 & 55 & 3 & \\
\hline & & 53 & & $9(5)$ & face & 110 & 1.55 & \\
\hline & & 54 & & 9 & 25.5 & 30,90 & 1.45 & \\
\hline & & 54 & & $1.75(7)$ & na & 165 & $r=28$ & \\
\hline & & 58 & & & $r=4$ & $r=18$ & & \\
\hline & & 50 & & & & & & \\
\hline & & 53 & & & & & & \\
\hline & & 50 & & & & & & \\
\hline & & 56 & & & & & & \\
\hline & & 56 & & & & & & \\
\hline & & 54 & & & & & & \\
\hline & & 56 & & & & & & \\
\hline & & 58 & & & & & & \\
\hline & & 56 & & & & & & \\
\hline & & 54 & & & & & & \\
\hline & & & & & & & & \\
\hline $\begin{array}{l}\text { Waypoint } \\
176\end{array}$ & & & & & & & & \\
\hline $\begin{array}{l}37.74168 \\
119.45834\end{array}$ & 2054 & 52 & -90 & $3(1)$ & 10 & 45 & 0.6 & $r=5$ \\
\hline & & 56 & & $>5(2)$ & 10 & 30,88 & 0.3 & \\
\hline & & 46 & & $4.5(3)$ & 30 & 125,90 & 0.2 & \\
\hline & & 56 & & $2.5(4)$ & 50 & 45,80 & 0.3 & \\
\hline & & 53 & & $1.75(5)$ & 130 & 115,90 & 0.1 & \\
\hline & & 55 & & $\begin{array}{l}?(6) \\
\end{array}$ & 15 & 15,86 & 1 & \\
\hline & & 49 & & $2(\mathrm{~F} 1)$ & 30 & 295 & 2 & \\
\hline & & 54 & & & $r=2$ & $r=14$ & 2 & \\
\hline & & 56 & & & & & 0.45 & \\
\hline & & 57 & & & & & $r=21$ & \\
\hline & & 54 & & & & & & \\
\hline & & 53 & & & & & & \\
\hline & & 58 & & & & & & \\
\hline & & 50 & & & & & & \\
\hline
\end{tabular}




\begin{tabular}{|c|c|c|c|c|c|c|c|c|}
\hline & & 56 & & & & & & \\
\hline & & 52 & & & & & & \\
\hline & & 58 & & & & & & \\
\hline & & 54 & & & & & & \\
\hline & & & & & & & & \\
\hline $\begin{array}{l}\text { Waypoint } \\
177\end{array}$ & & & & & & & & \\
\hline $\begin{array}{l}37.74172 \\
119.45769\end{array}$ & 2080 & 50 & -90 & $>4.5(1)$ & 10 & 20 & 0.1 & $r=6$ \\
\hline & & 52 & & $1(2)$ & 25 & 140 & \begin{tabular}{|l|}
0.01 \\
\end{tabular} & \\
\hline & & 50 & & $3(3)$ & 10 & 50 & 0.3 & \\
\hline & & 50 & & $0.5(4)$ & 10 & 180 & 0.02 & \\
\hline & & 51 & & $\begin{array}{l}0.2 \text { (Set } \\
1)\end{array}$ & 10 & 110 & 0.01 & \\
\hline & & 50 & & $1(1 \mathrm{~A})$ & 100 & 180 & 0.2 & \\
\hline & & 52 & & $\begin{array}{l}0.25 \\
(2 \mathrm{~A})\end{array}$ & 5 & 140 & 0.3 & \\
\hline & & 49 & & $1(3 \mathrm{~A})$ & 5 & 50 & 0.3 & \\
\hline & & 53 & & $0.2(4 \mathrm{~A})$ & $\sqrt{10}$ & 115 & 0.3 & \\
\hline & & 54 & & $2(1 \mathrm{~B})$ & 100 & 130,90 & 0.6 & \\
\hline & & 53 & & $1.5(2 \mathrm{~B})$ & 25 & 30,85 & 0.6 & \\
\hline & & 54 & & $2(3 \mathrm{~B})$ & 75 & 125,80 & 0.1 & \\
\hline & & 53 & & $6(1 \mathrm{C})$ & 4 & 25,80 & 0.01 & \\
\hline & & $\sqrt[55]{ }$ & & $1(2 \mathrm{C})$ & 3 & $\begin{array}{l}75 \\
75\end{array}$ & 0.6 & \\
\hline & & 52 & & 2 (1D) & 10 & 80 & 0.2 & \\
\hline & & & & $1(2 \mathrm{D})$ & 20 & 85 & 0.24 & \\
\hline & & & & $2(3 \mathrm{D})$ & 13 & 25 & 0.2 & \\
\hline & & & & $0.5(4 \mathrm{D})$ & 50 & 165 & $r=15$ & \\
\hline & & & & & 10 & $r=18$ & & \\
\hline & & & & & $r=4$ & & & \\
\hline & & & & & & & & \\
\hline $\begin{array}{l}\text { Waypoint } \\
178 \text { (LY3) }\end{array}$ & & & & & & & & \\
\hline $\begin{array}{l}37.74338 \\
119.45921\end{array}$ & 2016 & 52 & -90 & $3.5(1)$ & 0.1 & 35 & 1.2 & $r=6$ \\
\hline & & 62 & & $1.5(2)$ & face & 320,85 & 4 & \\
\hline & & 58 & & $4(3)$ & na & 30 & 3.5 & \\
\hline & & 52 & & $1(4)$ & 1 & \begin{tabular}{|l}
315 or \\
220,80 as \\
joint.
\end{tabular} & 5 & \\
\hline
\end{tabular}




\begin{tabular}{|c|c|c|c|c|c|c|c|c|}
\hline & & 56 & & $3(\mathrm{~F} 1)$ & 0.2 & 40 & $\mathbf{3 . 7 5}$ & \\
\hline & & 58 & & $1.5(\mathrm{~F} 2)$ & 10 & 290,90 & $r=30$ & \\
\hline & & 61 & & & 0.6 & $r=18$ & & \\
\hline & & 58 & & & $r=6$ & & & \\
\hline & & 60 & & & & & & \\
\hline & & 56 & & & & & & \\
\hline & & 58 & & & & & & \\
\hline & & 59 & & & & & & \\
\hline & & 61 & & & & & & \\
\hline & & 58 & & & & & & \\
\hline & & 50 & & & & & & \\
\hline & & 54 & & & & & & \\
\hline & & 52 & & & & & & \\
\hline & & 57 & & & & & & \\
\hline & & & & & & & & \\
\hline $\begin{array}{l}\text { Waypoint } \\
179\end{array}$ & & & & & & & & \\
\hline $\begin{array}{l}37.74372 \\
119.45856\end{array}$ & 2035 & 56 & -90 & $>20$ & na & 30 & 0.3 & $r=6$ \\
\hline & & 58 & & 1.5 & na & 80 & 0.6 & \\
\hline & & 55 & & 2 & na & 30 & 0.9 & \\
\hline & & 61 & & & $r=7$ & $r=20$ & 0.3 & \\
\hline & & 55 & & & & & 3 & \\
\hline & & 60 & & & & & 1.2 & \\
\hline & & 61 & & & & & 5 & \\
\hline & & 54 & & & & & 2 & \\
\hline & & 60 & & & & & 1.05 & \\
\hline & & $\overline{55}$ & & & & & $r=28$ & \\
\hline & & 57 & & & & & & \\
\hline & & 61 & & & & & & \\
\hline & & 52 & & & & & & \\
\hline & & 52 & & & & & & \\
\hline & & 54 & & & & & & \\
\hline & & 53 & & & & & & \\
\hline & & 54 & & & & & & \\
\hline & & 56 & & & & & & \\
\hline & & & & & & & & \\
\hline & & & & & & & & \\
\hline & & & & & & & & \\
\hline
\end{tabular}




\begin{tabular}{|c|c|c|c|c|c|c|c|c|}
\hline \multicolumn{9}{|l|}{$\begin{array}{l}\text { Waypoint } \\
180\end{array}$} \\
\hline $\begin{array}{l}37.74396 \\
119.45820\end{array}$ & 2041 & 55 & -45 & $3(1)$ & 6 & 75,50 & $r=21$ & $r=5$ \\
\hline & & 56 & & $5.5(2)$ & face & 75,90 & & \\
\hline & & 54 & & $3.5(3)$ & 1.5 & 55,87 & & \\
\hline & & 52 & & \begin{tabular}{|l|}
$1(4)$ \\
\end{tabular} & 2 & $\begin{array}{l}75 \\
5\end{array}$ & & \\
\hline & & $\sqrt[58]{ }$ & & \begin{tabular}{|l|l}
$5(\mathrm{~F} 1)$ \\
\end{tabular} & 10 & 140 & & \\
\hline & & 54 & & & 4 & $r=14$ & & \\
\hline & & 57 & & & $r=5$ & & & \\
\hline & & 56 & & & & & & \\
\hline & & 58 & & & & & & \\
\hline & & 58 & & & & & & \\
\hline & & 54 & & & & & & \\
\hline & & 56 & & & & & & \\
\hline & & 55 & & & & & & \\
\hline & & 57 & & & & & & \\
\hline & & 54 & & & & & & \\
\hline & & 55 & & & & & & \\
\hline & & 52 & & & & & & \\
\hline & & 55 & & & & & & \\
\hline & & & & & & & & \\
\hline $\begin{array}{l}\text { Waypoint } \\
181 \text { (LY4) }\end{array}$ & & & & & & & & \\
\hline $\begin{array}{l}37.74186 \\
119.45962\end{array}$ & 2035 & 54 & -45 & 3.5 & 25 & $\begin{array}{l}95,90 \text { or } \\
175 \\
\text { (flake) }\end{array}$ & 0.1 & $r=5$ \\
\hline & & 56 & & 2.5 & 10 & $\begin{array}{l}35,90 \text { or } \\
300 \\
\text { (flake) }\end{array}$ & 0.1 & \\
\hline & & 55 & & $0.7(1)$ & 10 & 345,55 & 0.2 & \\
\hline & & 51 & & $0.5(2)$ & 15 & 285 & 0.3 & \\
\hline & & 52 & & 1 (3) & 75 & 195 & 0.15 & \\
\hline & & 52 & & $0.7(4)$ & 10 & $\begin{array}{l}\text { measure } \\
\text { from } \\
\text { photos }\end{array}$ & 0.6 & \\
\hline & & 54 & & $3(5)$ & 10 & 340,60 & 0.2 & \\
\hline & & 54 & & $>3(\mathrm{~V} 1)$ & 100 & 255 & 0.3 & \\
\hline & & 58 & & & 10 & $r=9$ & 0.1 & \\
\hline
\end{tabular}




\begin{tabular}{|l|l|l|l|l|l|l|l|l|}
\hline & & 58 & & & $\boldsymbol{r}=\mathbf{4}$ & & 2 & \\
\hline & & 51 & & & & & 0.6 & \\
\hline & & 54 & & & & & 0.6 & \\
\hline & & 52 & & & & & $\mathbf{0 . 2 5}$ & \\
\hline & & 48 & & & & & $\boldsymbol{r}=\mathbf{1 5}$ & \\
\hline & & 54 & & & & & & \\
\hline & & 50 & & & & & & \\
\hline & & 55 & & & & & & \\
\hline & & $\mathbf{5 3}$ & & & & & & \\
\hline & & & & & & & & \\
\hline $\begin{array}{l}\text { Waypoint } \\
182\end{array}$ & & & & & & & & \\
\hline 37.74182 \\
119.45968
\end{tabular}




\begin{tabular}{|c|c|c|c|c|c|c|c|c|}
\hline & & 52 & & & $r=4$ & & 2 & \\
\hline & & 51 & & & & & 2 & \\
\hline & & 52 & & & & & 1.5 & \\
\hline & & 58 & & & & & 1.2 & \\
\hline & & 53 & & & & & $r=21$ & \\
\hline & & 52 & & & & & & \\
\hline & & 55 & & & & & & \\
\hline & & 54 & & & & & & \\
\hline & & 54 & & & & & & \\
\hline & & 59 & & & & & & \\
\hline & & 58 & & & & & & \\
\hline & & 53 & & & & & & \\
\hline & & 55 & & & & & & \\
\hline & & & & & & & & \\
\hline $\begin{array}{l}\text { Waypoint } \\
184\end{array}$ & & & & & & & & \\
\hline $\begin{array}{l}37.74741 \\
119.47275\end{array}$ & 1997 & 60 & -90 & $3(1)$ & 1 & 315 & 0.6 & $r=6$ \\
\hline & & 58 & & $>3.5(2)$ & 2 & 70,80 & 0.9 & \\
\hline & & 55 & & $3(3)$ & 1 & 20 & 0.6 & \\
\hline & & 56 & & $2.5(4)$ & 1 & 295 & 2.5 & \\
\hline & & 52 & & $>4(5)$ & 1 & 260 & 4 & \\
\hline & & 61 & & $>10(6)$ & $r=6$ & 275 & 2 & \\
\hline & & 60 & & & & $r=20$ & 1.2 & \\
\hline & & 52 & & & & & 1.2 & \\
\hline & & 58 & & & & & $r=28$ & \\
\hline & & 54 & & & & & & \\
\hline & & 64 & & & & & & \\
\hline & & 62 & & & & & & \\
\hline & & 64 & & & & & & \\
\hline & & 52 & & & & & & \\
\hline & & 62 & & & & & & \\
\hline & & 55 & & & & & & \\
\hline & & 56 & & & & & & \\
\hline & & 58 & & & & & & \\
\hline & & & & & & & & \\
\hline & & & & & & & & \\
\hline & & & & & & & & \\
\hline $\begin{array}{l}\text { Waypoint } \\
185 \text { (LY1) }\end{array}$ & & & & & & & & \\
\hline
\end{tabular}




\begin{tabular}{|c|c|c|c|c|c|c|c|c|}
\hline $\begin{array}{l}37.73752 \\
119.47859\end{array}$ & 1964 & 53 & -90 & $\begin{array}{l}\text { No } \\
\text { visible } \\
\text { joints. }\end{array}$ & $r=7$ & $r=20$ & $r=30$ & $r=7$ \\
\hline & & 56 & & & & & & \\
\hline & & 66 & & & & & & \\
\hline & & 54 & & & & & & \\
\hline & & 56 & & & & & & \\
\hline & & 60 & & & & & & \\
\hline & & 58 & & & & & & \\
\hline & & 60 & & & & & & \\
\hline & & 56 & & & & & & \\
\hline & & 64 & & & & & & \\
\hline & & 54 & & & & & & \\
\hline & & 52 & & & & & & \\
\hline & & 56 & & & & & & \\
\hline & & 58 & & & & & & \\
\hline & & 54 & & & & & & \\
\hline & & 59 & & & & & & \\
\hline & & 52 & & & & & & \\
\hline & & 57 & & & & & & \\
\hline & & & & & & & & \\
\hline $\begin{array}{l}\text { Waypoint } \\
186\end{array}$ & & & & & & & & \\
\hline $\begin{array}{l}37.73841 \\
119.47932\end{array}$ & 2018 & 54 & -90 & $5(\mathrm{~F} 1)$ & 100 & 90 & 0.3 & $r=6$ \\
\hline & & 53 & & $\begin{array}{l}1.75(\mathrm{~F} 2 \\
?)\end{array}$ & 25 & \begin{tabular}{|l}
250 or \\
045,90 as \\
joint
\end{tabular} & 0.6 & \\
\hline & & 49 & & $1.5(\mathrm{~F} 2)$ & 50 & 330 & 0.9 & \\
\hline & & 53 & & $2(\mathrm{~F} 3)$ & 50 & 115 & 4 & \\
\hline & & $\sqrt{55}$ & & $2(\mathrm{~F} 4)$ & 60 & 230 & 3 & \\
\hline & & 57 & & $0.5(1)$ & 5 & 220 & 0.9 & \\
\hline & & 53 & & $2.5(2)$ & 2.5 & 165 & 0.6 & \\
\hline & & 55 & & $>3(3)$ & 30 & 90,85 & 0.9 & \\
\hline & & $\sqrt{50}$ & & $>8(\mathrm{~V} 1)$ & 150 & 70 & $r=21$ & \\
\hline & & 54 & & $\begin{array}{l}1.75 \\
(\mathrm{~V} 2)\end{array}$ & 4 & 105 & & \\
\hline & & 56 & & $>4(\mathrm{~V} 3)$ & 100 & 225 & & \\
\hline & & $\sqrt{50}$ & & & 40 & $r=9$ & & \\
\hline & & 52 & & & $r=2$ & & & \\
\hline
\end{tabular}




\begin{tabular}{|l|l|l|l|l|l|l|l|l|}
\hline & & 58 & & & & & & \\
\hline & & 54 & & & & & & \\
\hline & & 56 & & & & & & \\
\hline & & 55 & & & & & & \\
\hline & & $\mathbf{5 4}$ & & & & & & \\
\hline & & & & & & & & \\
\hline $\begin{array}{l}\text { Waypoint } \\
187\end{array}$ & & & & & & & & \\
\hline $\begin{array}{l}37.73683 \\
119.47863\end{array}$ & 1959 & 50 & -90 & $2(1)$ & 2 & 355 & 0.6 & $\boldsymbol{r}=\mathbf{6}$ \\
\hline & & 54 & & $>3(2)$ & na & 100,80 & 1.5 & \\
\hline & & 54 & & $1.5(3)$ & na & 45 & 1.2 & \\
\hline & & 56 & & $4(4)$ & 4 & 270 & 2 & \\
\hline & & 56 & & $>3.5(5)$ & 3 & 350 & 1 & \\
\hline & & 52 & & $3(\mathrm{~F} 1)$ & 1 & 290 & $\mathbf{1 . 2}$ & \\
\hline & & 57 & & & $\boldsymbol{r}=\mathbf{6}$ & $\boldsymbol{r}=\mathbf{2 0}$ & $\boldsymbol{r}=\mathbf{2 8}$ & \\
\hline & & 58 & & & & & & \\
\hline & & 53 & & & & & & \\
\hline & & 53 & & & & & & \\
\hline & & 54 & & & & & & \\
\hline & & 56 & & & & & & \\
\hline & & 52 & & & & & & \\
\hline & & 56 & & & & & & \\
\hline & & 57 & & & & & & \\
\hline & & 55 & & & & & & \\
\hline & & 58 & & & & & & \\
\hline & & $\mathbf{5 5}$ & & & & & & \\
\hline
\end{tabular}




\begin{tabular}{|c|c|c|c|c|c|c|c|c|}
\hline \multicolumn{9}{|c|}{ San Joaquin River valley } \\
\hline \multicolumn{9}{|l|}{$\begin{array}{l}\text { Waypoint } \\
58 \text { (SJ4) }\end{array}$} \\
\hline Lat/Long & $\begin{array}{l}\text { Elev. } \\
(\mathrm{m})\end{array}$ & $\mathrm{SH}$ & Alpha & \begin{tabular}{|l} 
Joint \\
length \\
(m)
\end{tabular} & $\begin{array}{l}\text { Joint } \\
\text { width } \\
(\mathrm{mm}) \\
\end{array}$ & $\begin{array}{l}\text { Spacing } \\
\text { between } \\
\text { joints (m) }\end{array}$ & $\begin{array}{l}\text { Joint strike } \\
\text { and dip }\end{array}$ & Continuity \\
\hline \multirow[t]{18}{*}{$\begin{array}{l}37.51050 \\
119.19923 \\
\end{array}$} & 1402 & 36 & -90 & $\begin{array}{l}\text { No } \\
\text { visible } \\
\text { joints. }\end{array}$ & $r=7$ & $r=30$ & $r=20$ & $r=7$ \\
\hline & & 62 & & & & & & \\
\hline & & 56 & & & & & & \\
\hline & & 42 & & & & & & \\
\hline & & 42 & & & & & & \\
\hline & & 65 & & & & & & \\
\hline & & 64 & & & & & & \\
\hline & & 33 & & & & & & \\
\hline & & 57 & & & & & & \\
\hline & & 57 & & & & & & \\
\hline & & 63 & & & & & & \\
\hline & & 59 & & & & & & \\
\hline & & 60 & & & & & & \\
\hline & & 42 & & & & & & \\
\hline & & 46 & & & & & & \\
\hline & & 61 & & & & & & \\
\hline & & 58 & & & & & & \\
\hline & & 53 & & & & & & \\
\hline & & & & & & & & \\
\hline \multicolumn{9}{|l|}{\begin{tabular}{|l} 
Waypoint \\
59 \\
\end{tabular}} \\
\hline $\begin{array}{l}37.51066 \\
119.19910\end{array}$ & 1404 & 44 & -90 & $\begin{array}{l}\text { No } \\
\text { visible } \\
\text { jointing. }\end{array}$ & $r=7$ & $r=30$ & $r=20$ & $r=7$ \\
\hline & & 62 & & & & & & \\
\hline & & 66 & & & & & & \\
\hline & & 68 & & & & & & \\
\hline & & 50 & & & & & & \\
\hline & & 64 & & & & & & \\
\hline & & 53 & & & & & & \\
\hline & & 44 & & & & & & \\
\hline
\end{tabular}




\begin{tabular}{|c|c|c|c|c|c|c|c|c|}
\hline & & 62 & & & & & & \\
\hline & & 56 & & & & & & \\
\hline & & 64 & & & & & & \\
\hline & & 41 & & & & & & \\
\hline & & 58 & & & & & & \\
\hline & & 53 & & & & & & \\
\hline & & 59 & & & & & & \\
\hline & & 68 & & & & & & \\
\hline & & 58 & & & & & & \\
\hline & & 57 & & & & & & \\
\hline & & & & & & & & \\
\hline $\begin{array}{l}\text { Waypoint } \\
60\end{array}$ & & & & & & & & \\
\hline $\begin{array}{l}37.51073 \\
119.19906\end{array}$ & 1405 & 52 & -90 & 10 & 2 & 0.50 & 206,82 & $r=6$ \\
\hline & & 64 & & 0.5 & 10 & 0.30 & 306,76 & \\
\hline & & 44 & & 0.3 & 50.8 & 0.60 & $r=14$ & \\
\hline & & 58 & & 1 & 25 & 0.47 & & \\
\hline & & 34 & & 1.3 & 21.95 & $r=21$ & & \\
\hline & & 56 & & 1 & 17.5 & & & \\
\hline & & 48 & & 0.1 & $r=7$ & & & \\
\hline & & 50 & & & & & & \\
\hline & & 50 & & & & & & \\
\hline & & 64 & & & & & & \\
\hline & & 38 & & & & & & \\
\hline & & 39 & & & & & & \\
\hline & & 40 & & & & & & \\
\hline & & 41 & & & & & & \\
\hline & & 36 & & & & & & \\
\hline & & 49 & & & & & & \\
\hline & & 54 & & & & & & \\
\hline & & 48 & & & & & & \\
\hline & & & & & & & & \\
\hline $\begin{array}{l}\text { Waypoint } \\
61\end{array}$ & & & & & & & & \\
\hline $\begin{array}{l}37.51076 \\
119.19904\end{array}$ & 1410 & 50 & -90 & 1.5 & na & 0.25 & 359,63 & $r=5$ \\
\hline & & 38 & & 0.8 & na & 0.15 & 6 & \\
\hline & & 49 & & 1 & 150 & 0.90 & 19 & \\
\hline & & 33 & & 2.4 & 50 & 0.50 & $r=9$ & \\
\hline
\end{tabular}




\begin{tabular}{|c|c|c|c|c|c|c|c|c|}
\hline & & 42 & & 2.5 & $r=5$ & 0.90 & & \\
\hline & & 60 & & 2 & & 0.54 & & \\
\hline & & 38 & & 1.75 & & 0.50 & & \\
\hline & & 54 & & 0.18 & & $r=21$ & & \\
\hline & & 38 & & & & & & \\
\hline & & 56 & & & & & & \\
\hline & & 58 & & & & & & \\
\hline & & 52 & & & & & & \\
\hline & & 44 & & & & & & \\
\hline & & 62 & & & & & & \\
\hline & & 42 & & & & & & \\
\hline & & 52 & & & & & & \\
\hline & & 40 & & & & & & \\
\hline & & 48 & & & & & & \\
\hline & & & & & & & & \\
\hline $\begin{array}{l}\text { Waypoint } \\
62\end{array}$ & & & & & & & & \\
\hline $\begin{array}{l}37.51082 \\
119.19893\end{array}$ & 1420 & 63 & -90 & 0.4 & 76 & 0.20 & $\begin{array}{l}169,85 / 165 \\
, 64\end{array}$ & $r=5$ \\
\hline & & 69 & & 0.5 & 76 & 1.50 & 205,85 & \\
\hline & & 59 & & 3.5 & 0.1 & 0.15 & 195,84 & \\
\hline & & 56 & & 3 & 0.1 & 0.62 & 97,89 & \\
\hline & & 64 & & 1 & 0.1 & 0.20 & 19,90 & \\
\hline & & 62 & & 1.5 & 30.46 & $r=15$ & $r=14$ & \\
\hline & & 61 & & 1.3 & 0.1 & & & \\
\hline & & 45 & & 2 & $r=6$ & & & \\
\hline & & 52 & & 1.4 & & & & \\
\hline & & $\overline{55}$ & & 0.14 & & & & \\
\hline & & 62 & & & & & & \\
\hline & & 57 & & & & & & \\
\hline & & 42 & & & & & & \\
\hline & & 39 & & & & & & \\
\hline & & 48 & & & & & & \\
\hline & & 50 & & & & & & \\
\hline & & 65 & & & & & & \\
\hline & & 53 & & & & & & \\
\hline & & 56 & & & & & & \\
\hline & & & & & & & & \\
\hline & & & & & & & & \\
\hline & & & & & & & & \\
\hline
\end{tabular}




\begin{tabular}{|c|c|c|c|c|c|c|c|c|}
\hline \multicolumn{9}{|l|}{$\begin{array}{l}\text { Waypoint } \\
63\end{array}$} \\
\hline \begin{tabular}{|l|}
37.51093 \\
119.19892
\end{tabular} & 1416 & 52 & -90 & 1 & neg & 1.80 & 15,90 & $r=6$ \\
\hline & & 66 & & 2 & neg & 0.30 & 10,100 & \\
\hline & & 34 & & 2.5 & 20 & 0.60 & 21,76 & \\
\hline & & \begin{tabular}{|l|}
58 \\
\end{tabular} & & 10 & $r=4$ & 0.90 & $r=9$ & \\
\hline & & 4 & & 2.25 & & 0.60 & & \\
\hline & & 59 & & 0.225 & & $r=21$ & & \\
\hline & & 59 & & & & & & \\
\hline & & \begin{tabular}{|l|}
54 \\
\end{tabular} & & & & & & \\
\hline & & 54 & & & & & & \\
\hline & & 52 & & & & & & \\
\hline & & 32 & & & & & & \\
\hline & & 48 & & & & & & \\
\hline & & \begin{tabular}{|l|}
54 \\
\end{tabular} & & & & & & \\
\hline & & 52 & & & & & & \\
\hline & & 42 & & & & & & \\
\hline & & 54 & & & & & & \\
\hline & & 48 & & & & & & \\
\hline & & \begin{tabular}{|l|}
51 \\
\end{tabular} & & & & & & \\
\hline & & & & & & & & \\
\hline $\begin{array}{l}\text { Waypoint } \\
64\end{array}$ & & & & & & & & \\
\hline $\begin{array}{l}37.51105 \\
119.19889\end{array}$ & 1422 & 51 & -45 & $20(1)$ & 10 & 0.30 & 17,88 & \\
\hline & & $\sqrt[52]{2}$ & & $15(2)$ & 20 & 0.15 & 23,75 & \\
\hline & & 42 & & & 15 & 0.50 & $r=14$ & $r=5$ \\
\hline & & 45 & & & $r=4$ & \begin{tabular}{|l|l|}
0.32 \\
\end{tabular} & & \\
\hline & & 39 & & & & 0.31 & & \\
\hline & & 46 & & & & $r=15$ & & \\
\hline & & 32 & & & & & & \\
\hline & & 52 & & & & & & \\
\hline & & 52 & & & & & & \\
\hline & & 41 & & & & & & \\
\hline & & 54 & & & & & & \\
\hline & & \begin{tabular}{|l|}
54 \\
\end{tabular} & & & & & & \\
\hline & & 44 & & & & & & \\
\hline & & 29 & & & & & & \\
\hline & & 37 & & & & & & \\
\hline
\end{tabular}




\begin{tabular}{|c|c|c|c|c|c|c|c|c|}
\hline & & 54 & & & & & & \\
\hline & & 45 & & & & & & \\
\hline & & 45 & & & & & & \\
\hline & & & & & & & & \\
\hline \multicolumn{9}{|l|}{$\begin{array}{l}\text { Waypoint } \\
65 \text { (SJ3) }\end{array}$} \\
\hline \multirow[t]{18}{*}{$\begin{array}{l}37.51120 \\
119.20140 \\
\end{array}$} & 1363 & 42 & -90 & 25 & 10 & 0.20 & 0 & $r=5$ \\
\hline & & 51 & & 15 & 100 & 1.20 & 191,72 & \\
\hline & & 32 & & 10 & 20 & 1.80 & 99 & \\
\hline & & 48 & & 1 & 15 & 1.07 & $r=14$ & \\
\hline & & 42 & & 1.5 & 50 & 1.20 & & \\
\hline & & 54 & & 1.5 & 39 & $r=28$ & & \\
\hline & & 37 & & 5.75 & 20 & & & \\
\hline & & 62 & & 1 & $r=4$ & & & \\
\hline & & 45 & & & & & & \\
\hline & & 38 & & & & & & \\
\hline & & 35 & & & & & & \\
\hline & & 48 & & & & & & \\
\hline & & 38 & & & & & & \\
\hline & & 40 & & & & & & \\
\hline & & 30 & & & & & & \\
\hline & & 38 & & & & & & \\
\hline & & 51 & & & & & & \\
\hline & & 43 & & & & & & \\
\hline & & & & & & & & \\
\hline \multicolumn{9}{|l|}{$\begin{array}{l}\text { Waypoint } \\
66\end{array}$} \\
\hline $\begin{array}{l}37.50979 \\
119.20454\end{array}$ & 1388 & 40 & -90 & 6 & 152 & 0.15 & $\begin{array}{l}350,35 \\
\text { (joint set } \\
\text { roughly } \\
\text { equivalent) }\end{array}$ & $r=5$ \\
\hline & & 50 & & 0.3 & 30 & 0.30 & $r=14$ & \\
\hline & & 4 & & 0.3 & 20 & 1.80 & & \\
\hline & & 44 & & 0.3 & 40 & 1.50 & & \\
\hline & & 30 & & 0.03 & 60.5 & 0.90 & & \\
\hline & & 4 & & & 35 & 0.93 & & \\
\hline & & 30 & & & $r=5$ & 0.90 & & \\
\hline & & 28 & & & & $r=21$ & & \\
\hline
\end{tabular}




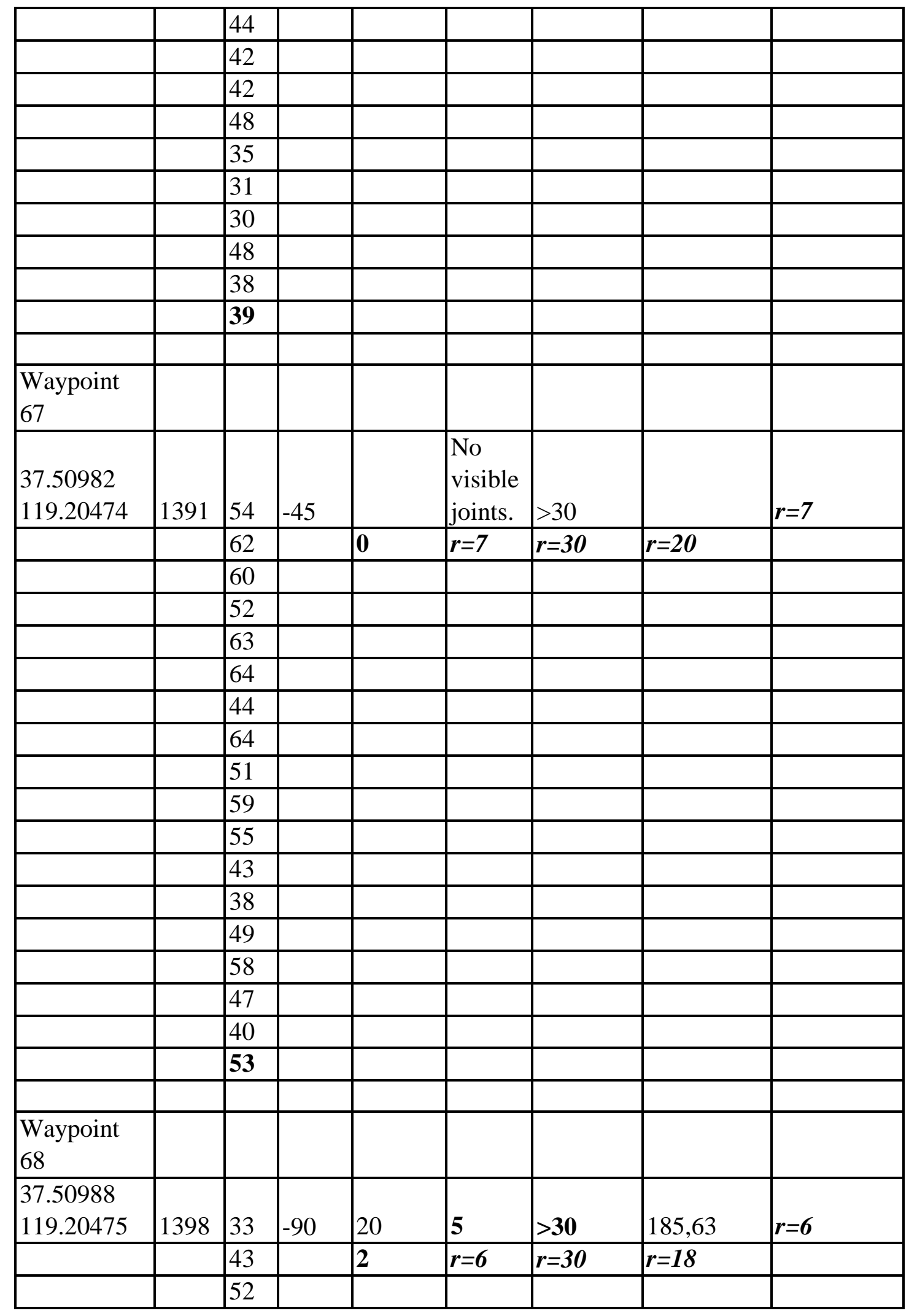




\begin{tabular}{|l|l|l|l|l|l|l|l|}
\hline & & 58 & & & & & \\
\hline & & 52 & & & & & \\
\hline \\
\hline
\end{tabular}




\begin{tabular}{|c|c|c|c|c|c|c|c|c|}
\hline \multicolumn{9}{|l|}{$\begin{array}{l}\text { Waypoint } \\
69\end{array}$} \\
\hline $\begin{array}{l}37.50995 \\
119.20486\end{array}$ & 1405 & 34 & -90 & $\begin{array}{l}>20 \\
\text { (closed) }\end{array}$ & 15 & $>30$ & 205,35 & $r=6$ \\
\hline & & 42 & & 0 & $r=5$ & $r=30$ & $r=18$ & \\
\hline & & 43 & & & & & & \\
\hline & & 53 & & & & & & \\
\hline & & 54 & & & & & & \\
\hline & & 56 & & & & & & \\
\hline & & 52 & & & & & & \\
\hline & & 55 & & & & & & \\
\hline & & 33 & & & & & & \\
\hline & & 46 & & & & & & \\
\hline & & 33 & & & & & & \\
\hline & & 35 & & & & & & \\
\hline & & 48 & & & & & & \\
\hline & & 52 & & & & & & \\
\hline & & 35 & & & & & & \\
\hline & & 49 & & & & & & \\
\hline & & 52 & & & & & & \\
\hline & & 45 & & & & & & \\
\hline & & & & & & & & \\
\hline $\begin{array}{l}\text { Waypoint } \\
70 \text { (SJ4) }\end{array}$ & & & & & & & & \\
\hline $\begin{array}{l}37.51024 \\
119.199528\end{array}$ & 1392 & 60 & -90 & 6 & 35 & 2.00 & $\begin{array}{l}281,78 \\
\text { (lower) }\end{array}$ & $r=5$ \\
\hline & & 62 & & 2 & 76 & 0.20 & $\begin{array}{l}193,58 \\
\text { (left) }\end{array}$ & \\
\hline & & 60 & & 0.2 & 1 & 0.30 & $\begin{array}{l}38,89 \\
\text { (right) }\end{array}$ & \\
\hline & & 64 & & 0.3 & 2 & 0.30 & $\begin{array}{l}277,69 \\
\text { (upper) }\end{array}$ & \\
\hline & & $\sqrt{60}$ & & 1.15 & 152 & $\overline{0.50}$ & $r=14$ & \\
\hline & & 63 & & 0.115 & 53.2 & 1.50 & & \\
\hline & & 64 & & & 35 & 0.05 & & \\
\hline & & 70 & & & $r=2$ & 0.07 & & \\
\hline & & 60 & & & & 0.05 & & \\
\hline & & 58 & & & & 0.07 & & \\
\hline
\end{tabular}




\begin{tabular}{|c|c|c|c|c|c|c|c|c|}
\hline & & 50 & & & & 0.07 & & \\
\hline & & 66 & & & & 0.07 & & \\
\hline & & 65 & & & & 0.05 & & \\
\hline & & 65 & & & & 0.06 & & \\
\hline & & 61 & & & & 0.05 & & \\
\hline & & 60 & & & & 0.07 & & \\
\hline & & 64 & & & & 0.34 & & \\
\hline & & 62 & & & & 0.07 & & \\
\hline & & & & & & $r=15$ & & \\
\hline $\begin{array}{l}\text { Waypoint } \\
71\end{array}$ & & & & & & & & \\
\hline $\begin{array}{l}37.51012 \\
119.19937\end{array}$ & 1405 & 52 & -90 & 2 & 76 & 0.07 & 201,74 & 5 \\
\hline & & 52 & & 2 & 250 & 0.50 & 304,79 & \\
\hline & & 36 & & 1.5 & 100 & 1.20 & \begin{tabular}{|l|} 
roughly \\
parallel to \\
joint 1
\end{tabular} & $r=5$ \\
\hline & & 66 & & 1.2 & 100 & 1.20 & $r=14$ & \\
\hline & & 48 & & 1.5 & 250 & 1.20 & & \\
\hline & & 65 & & 25 & 20 & 1.00 & & \\
\hline & & 52 & & 1.75 & 10 & 1.00 & & \\
\hline & & 65 & & 0.175 & 10 & 0.15 & & \\
\hline & & 70 & & & 15 & 0.15 & & \\
\hline & & 44 & & & 0.5 & 0.20 & & \\
\hline & & 64 & & & 0.5 & 0.20 & & \\
\hline & & 56 & & & \begin{tabular}{|l|}
75.64 \\
\end{tabular} & 0.30 & & \\
\hline & & 66 & & & 47.82 & 1.50 & & \\
\hline & & 54 & & & $r=4$ & 1.30 & & \\
\hline & & 66 & & & & 0.71 & & \\
\hline & & 55 & & & & 0.75 & & \\
\hline & & 52 & & & & $r=21$ & & \\
\hline & & 57 & & & & & & \\
\hline & & & & & & & & \\
\hline & & & & & & & & \\
\hline & & & & & & & & \\
\hline $\begin{array}{l}\text { Waypoint } \\
72\end{array}$ & & & & & & & & \\
\hline $\begin{array}{l}37.50989 \\
119.19945\end{array}$ & 1426 & 58 & -90 & 25 & 300 & 0.15 & 304,39 & $r=6$ \\
\hline & & 60 & & 4 & 127 & 2.00 & \begin{tabular}{|l|}
105,83 \\
\end{tabular} & \\
\hline
\end{tabular}




\begin{tabular}{|c|c|c|c|c|c|c|c|c|}
\hline & & 47 & & 4 & 20 & 0.60 & $r=14$ & \\
\hline & & 50 & & 0.5 & 10 & 3.50 & & \\
\hline & & 50 & & 1 & 20 & 1.20 & & \\
\hline & & 60 & & 4 & 100 & 0.30 & & \\
\hline & & 50 & & 0.4 & 30 & 1.50 & & \\
\hline & & 56 & & & 87 & 1.50 & & \\
\hline & & 50 & & & 30 & 0.30 & & \\
\hline & & 56 & & & $r=2$ & 1.23 & & \\
\hline & & 58 & & & & 1.20 & & \\
\hline & & 54 & & & & $r=21$ & & \\
\hline & & 52 & & & & & & \\
\hline & & 64 & & & & & & \\
\hline & & 45 & & & & & & \\
\hline & & 38 & & & & & & \\
\hline & & 50 & & & & & & \\
\hline & & 53 & & & & & & \\
\hline & & & & & & & & \\
\hline $\begin{array}{l}\text { Waypoint } \\
73\end{array}$ & & & & & & & & \\
\hline $\begin{array}{l}37.50974 \\
119.19944\end{array}$ & 1440 & 54 & -90 & 20 & 64 & 0.50 & 281,32 & $r=5$ \\
\hline & & 55 & & 1.5 & 20 & 0.60 & 10,84 & \\
\hline & & $\overline{55}$ & & 1 & 10 & 1.80 & 12,80 & \\
\hline & & 4 & & 2 & 20 & 2.50 & 290,25 & \\
\hline & & 56 & & 2.5 & 100 & 1.80 & $r=14$ & \\
\hline & & $\overline{59}$ & & 2.5 & 150 & 1.50 & & \\
\hline & & 42 & & 1.8 & $\sqrt{80}$ & 0.60 & & \\
\hline & & 54 & & 2 & 100 & 1.40 & & \\
\hline & & 58 & & 2 & 30 & 2.00 & & \\
\hline & & 60 & & 1.5 & 20 & 1.41 & & \\
\hline & & 50 & & 2 & 10 & 1.50 & & \\
\hline & & 60 & & 0.2 & 55 & $r=28$ & & \\
\hline & & 53 & & & 30 & & & \\
\hline & & 64 & & & $r=2$ & & & \\
\hline & & 48 & & & & & & \\
\hline & & 52 & & & & & & \\
\hline & & 63 & & & & & & \\
\hline & & 55 & & & & & & \\
\hline & & & & & & & & \\
\hline
\end{tabular}




\begin{tabular}{|c|c|c|c|c|c|c|c|c|}
\hline \multicolumn{9}{|c|}{\begin{tabular}{|l|l} 
Waypoint & \\
smoking & \\
crack & \\
(Double & \\
check all & \\
previous & \\
waypoints) & \\
\end{tabular}} \\
\hline & 1450 & 45 & -45 & $3.5(1)$ & 5 & 1.50 & 353,48 & $r-6$ \\
\hline & & 44 & & $>9(2)$ & 17.8 & 1.50 & 11,45 & \\
\hline & & 53 & & $3(3)$ & 6 & 2.00 & 35, na & \\
\hline & & 55 & & $>20(4)$ & 10 & 1.00 & 6,64 & \\
\hline & & 40 & & $1(5)$ & 4 & 1.00 & 110,67 & \\
\hline & & 52 & & & 8.56 & 3.00 & $r=21$ & \\
\hline & & 46 & & & 6 & 1.67 & & \\
\hline & & 52 & & & $r=4$ & 1.50 & & \\
\hline & & 58 & & & & $r=28$ & & \\
\hline & & 64 & & & & & & \\
\hline & & 40 & & & & & & \\
\hline & & 48 & & & & & & \\
\hline & & 38 & & & & & & \\
\hline & & 45 & & & & & & \\
\hline & & 54 & & & & & & \\
\hline & & 24 & & & & & & \\
\hline & & 42 & & & & & & \\
\hline & & 47 & & & & & & \\
\hline & & & & & & & & \\
\hline & & & & & & & & \\
\hline $\begin{array}{l}\text { Waypoint } \\
74 \text { (SJ2) }\end{array}$ & & & & & & & & \\
\hline \begin{tabular}{|l|}
37.49036 \\
119.20627
\end{tabular} & 1343 & 49 & -90 & 2.5 & 100 & 0.75 & 135,45 & $r=5$ \\
\hline & & 56 & & 1 & 50 & 0.50 & 242,49 & \\
\hline & & 52 & & 1.5 & 150 & \begin{tabular}{|l|}
0.90 \\
\end{tabular} & $r=9$ & \\
\hline & & 41 & & 2 & 100 & \begin{tabular}{|l|}
1.00 \\
\end{tabular} & & \\
\hline & & 56 & & 1.75 & 100 & 0.75 & & \\
\hline & & 54 & & \begin{tabular}{|l|l|}
0.175 \\
\end{tabular} & 100 & \begin{tabular}{|l|}
1.00 \\
\end{tabular} & & \\
\hline & & 50 & & & $r=2$ & 0.82 & & \\
\hline & & 44 & & & & \begin{tabular}{|l|}
0.83 \\
\end{tabular} & & \\
\hline & & 48 & & & & $r=21$ & & \\
\hline & & 45 & & & & & & \\
\hline
\end{tabular}




\begin{tabular}{|l|l|l|l|l|l|l|l|l|}
\hline & & 58 & & & & & & \\
\hline & & 34 & & & & & & \\
\hline & & 58 & & & & & & \\
\hline & & 56 & & & & & & \\
\hline & & 58 & & & & & & \\
\hline & & 47 & & & & & & \\
\hline & & 62 & & & & & & \\
\hline & & $\mathbf{5 1}$ & & & & & & \\
\hline & & & & & & & & \\
\hline $\begin{array}{l}\text { Waypoint } \\
75\end{array}$ & & & & & & & & \\
\hline 37.49047 \\
119.20604
\end{tabular}




\begin{tabular}{|l|l|l|l|l|l|l|l|l|}
\hline & & 48 & & & & & & \\
\hline & & 56 & & & & & & \\
\hline & & 49 & & & & & & \\
\hline & & 52 & & & & & & \\
\hline & & 67 & & & & & & \\
\hline & & 54 & & & & & & \\
\hline & & 60 & & & & & & \\
\hline & & 50 & & & & & & \\
\hline & & 56 & & & & & & \\
\hline & & 56 & & & & & & \\
\hline & & 45 & & & & & & \\
\hline & & 51 & & & & & & \\
\hline & & & & & & & & \\
\hline Waypoint & & & & & & & & \\
\hline 77 & & & & & & & & \\
\hline & & & & & & & & \\
\hline \\
\hline
\end{tabular}




\begin{tabular}{|c|c|c|c|c|c|c|c|c|}
\hline $\begin{array}{l}\text { Waypoint } \\
78\end{array}$ & & & & & & & & \\
\hline $\begin{array}{l}37.49072 \\
119.20548\end{array}$ & 1393 & 63 & -45 & 20 & 200 & $>30$ & 287,65 & $r=7$ \\
\hline & & 55 & & 2 & $r=4$ & $r=28$ & $r=14$ & \\
\hline & & 38 & & & & & & \\
\hline & & 40 & & & & & & \\
\hline & & 48 & & & & & & \\
\hline & & 46 & & & & & & \\
\hline & & 50 & & & & & & \\
\hline & & 60 & & & & & & \\
\hline & & 51 & & & & & & \\
\hline & & 60 & & & & & & \\
\hline & & 48 & & & & & & \\
\hline & & 61 & & & & & & \\
\hline & & 51 & & & & & & \\
\hline & & 49 & & & & & & \\
\hline & & 53 & & & & & & \\
\hline & & 61 & & & & & & \\
\hline & & 54 & & & & & & \\
\hline & & 52 & & & & & & \\
\hline & & & & & & & & \\
\hline $\begin{array}{l}\text { Waypoint } \\
79\end{array}$ & & & & & & & & \\
\hline $\begin{array}{l}37.4907 \\
119.20531\end{array}$ & 1405 & 28 & -90 & $1(1)$ & 50 & 0.20 & 15 , na & $r=5$ \\
\hline & & 36 & & $2(2)$ & 40 & 0.75 & 18, na & \\
\hline & & 40 & & $4(3)$ & 2 & 0.90 & 180,25 & \\
\hline & & 42 & & $\begin{array}{l}\text { Closed } \\
\text { or less } \\
\text { than } \\
0.05 \mathrm{~m} \\
\text { wide. }\end{array}$ & 30.667 & 1.00 & $r=14$ & \\
\hline & & 36 & & 1 & 40 & 0.71 & & \\
\hline & & 42 & & 2 & $r=4$ & 0.83 & & \\
\hline & & 32 & & 2 & & $r=21$ & & \\
\hline & & 42 & & 2.5 & & & & \\
\hline & & 34 & & 4 & & & & \\
\hline & & 30 & & 4 & & & & \\
\hline & & 42 & & 2.5 & & & & \\
\hline
\end{tabular}




\begin{tabular}{|c|c|c|c|c|c|c|c|c|}
\hline & & 44 & & 2.5 & & & & \\
\hline & & 42 & & 0.25 & & & & \\
\hline & & 40 & & & & & & \\
\hline & & 38 & & & & & & \\
\hline & & 38 & & & & & & \\
\hline & & 44 & & & & & & \\
\hline & & 38 & & & & & & \\
\hline & & & & & & & & \\
\hline $\begin{array}{l}\text { Waypoint } \\
80\end{array}$ & & & & & & & & \\
\hline $\begin{array}{l}37.49085 \\
119.20469\end{array}$ & 1439 & 62 & -90 & 3 & 40 & 0.50 & 104,65 & $r=6$ \\
\hline & & 70 & & 3.5 & 0 & \begin{tabular}{|l|}
0.30 \\
\end{tabular} & 42,85 & \\
\hline & & 50 & & 3.5 & 127 & 0.30 & 110 & \\
\hline & & 54 & & 3 & 25 & 0.90 & 310,30 & \\
\hline & & 46 & & 3 & 20 & \begin{tabular}{|l|}
1.20 \\
\end{tabular} & 220,80 & \\
\hline & & 62 & & 3.5 & 53 & \begin{tabular}{|l|}
0.30 \\
\end{tabular} & $r=14$ & \\
\hline & & 58 & & 1 & 32.5 & 1.50 & & \\
\hline & & 64 & & 0.8 & $r=2$ & 1.00 & & \\
\hline & & 44 & & 1 & & \begin{tabular}{|l|}
0.75 \\
\end{tabular} & & \\
\hline & & 44 & & 0.9 & & \begin{tabular}{|l|}
0.70 \\
\end{tabular} & & \\
\hline & & 36 & & 0.8 & & $r=21$ & & \\
\hline & & $\sqrt[58]{ }$ & & 3 & & & & \\
\hline & & 48 & & 2 & & & & \\
\hline & & 42 & & 3 & & & & \\
\hline & & 56 & & 2.5 & & & & \\
\hline & & 34 & & 3 & & & & \\
\hline & & 36 & & 3 & & & & \\
\hline & & 51 & & 0.3 & & & & \\
\hline & & & & & & & & \\
\hline $\begin{array}{l}\text { Waypoint } \\
81\end{array}$ & & & & & & & & \\
\hline $\begin{array}{l}37.48689 \\
119.20548\end{array}$ & & & & & & & & \\
\hline $\begin{array}{l}\text { Waypoint } \\
82 \text { (SJ1) }\end{array}$ & & & & & & & & \\
\hline $\begin{array}{l}37.4823 \\
119.20902\end{array}$ & 1377 & 36 & -90 & 3.5 & 0 & 1.00 & 276,76 & $r=6$ \\
\hline & & 24 & & 1 & 0 & 3.50 & 9.51 & \\
\hline
\end{tabular}




\begin{tabular}{|c|c|c|c|c|c|c|c|c|}
\hline & & 41 & & 0.9 & 0 & 2.50 & 159,75 & \\
\hline & & 53 & & 0.5 & $r=6$ & 1.50 & $r=18$ & \\
\hline & & 56 & & 2 & & 1.00 & & \\
\hline & & 52 & & 1 & & 1.80 & & \\
\hline & & 54 & & & & 2.00 & & \\
\hline & & $\overline{58}$ & & & & 1.80 & & \\
\hline & & 41 & & & & 1.89 & & \\
\hline & & \begin{tabular}{|l|}
54 \\
\end{tabular} & & & & 1.80 & & \\
\hline & & 65 & & & & $r=28$ & & \\
\hline & & 65 & & & & & & \\
\hline & & 53 & & & & & & \\
\hline & & 60 & & & & & & \\
\hline & & 55 & & & & & & \\
\hline & & 61 & & & & & & \\
\hline & & 44 & & & & & & \\
\hline & & 51 & & & & & & \\
\hline & & & & & & & & \\
\hline $\begin{array}{l}\text { Waypoint } \\
83\end{array}$ & & & & & & & & \\
\hline $\begin{array}{l}37.48283 \\
119.20913\end{array}$ & 1380 & 63 & -90 & $1(1)$ & 0 & 0.30 & 258,65 & $r=6$ \\
\hline & & 61 & & $3.5(2)$ & 0 & 5.00 & 295 & \\
\hline & & 52 & & $>20(3)$ & 0 & 3.00 & 261 & \\
\hline & & 68 & & & $r=7$ & 2.77 & $r=20$ & \\
\hline & & 49 & & 0 & & $r=28$ & & \\
\hline & & 38 & & & & & & \\
\hline & & 52 & & & & & & \\
\hline & & 60 & & & & & & \\
\hline & & 45 & & & & & & \\
\hline & & 60 & & & & & & \\
\hline & & 42 & & & & & & \\
\hline & & 55 & & & & & & \\
\hline & & 52 & & & & & & \\
\hline & & 48 & & & & & & \\
\hline & & 32 & & & & & & \\
\hline & & 51 & & & & & & \\
\hline & & 52 & & & & & & \\
\hline & & 52 & & & & & & \\
\hline & & & & & & & & \\
\hline
\end{tabular}




\begin{tabular}{|c|c|c|c|c|c|c|c|c|}
\hline \multicolumn{9}{|l|}{$\begin{array}{l}\text { Waypoint } \\
84\end{array}$} \\
\hline $\begin{array}{l}37.48288 \\
119.20919\end{array}$ & 1389 & 66 & -90 & 9 & 76 & 0.20 & 259,79 & $r=6$ \\
\hline & & 68 & & 0.3 & 20 & 0.70 & 261,65 & \\
\hline & & 68 & & 0.2 & 25 & 1.20 & 278,70 & \\
\hline & & 66 & & 1.2 & 30 & 1.30 & $r=14$ & \\
\hline & & 72 & & 1.5 & 40 & 1.50 & & \\
\hline & & 70 & & 1.2 & 100 & 0.60 & & \\
\hline & & 70 & & 0.12 & 150 & 0.92 & & \\
\hline & & 69 & & & 63 & 0.95 & & \\
\hline & & 68 & & & 40 & $r=21$ & & \\
\hline & & 66 & & & $r=4$ & & & \\
\hline & & 71 & & & & & & \\
\hline & & 66 & & & & & & \\
\hline & & 70 & & & & & & \\
\hline & & 70 & & & & & & \\
\hline & & 71 & & & & & & \\
\hline & & 72 & & & & & & \\
\hline & & 65 & & & & & & \\
\hline & & 69 & & & & & & \\
\hline & & & & & & & & \\
\hline $\begin{array}{l}\text { Waypoint } \\
85\end{array}$ & & & & & & & & \\
\hline $\begin{array}{l}37.48298 \\
119.20928\end{array}$ & 1395 & 46 & -90 & 2.5 & 150 & 0.20 & 34,72 & $r=6$ \\
\hline & & 47 & & 12 & 120 & 0.60 & 164,60 & \\
\hline & & 43 & & 12 & 100 & 1.20 & 329,45 & \\
\hline & & 42 & & 1 & face(?) & 1.00 & 23,90 & \\
\hline & & 42 & & 2 & face(?) & 1.20 & 282,85 & \\
\hline & & 44 & & 4 & face(?) & 1.00 & 138,44 & \\
\hline & & 41 & & 1.5 & 100 & 0.87 & 346,59 & \\
\hline & & 60 & & 10 & 25 & 1.00 & 251 & \\
\hline & & 40 & & 3.25 & 200 & $r=21$ & 339,59 & \\
\hline & & 56 & & 0.325 & 116 & & $r=14$ & \\
\hline & & 43 & & & 110 & & & \\
\hline & & 41 & & & $r=2$ & & & \\
\hline & & 38 & & & & & & \\
\hline & & 58 & & & & & & \\
\hline & & 46 & & & & & & \\
\hline
\end{tabular}




\begin{tabular}{|c|c|c|c|c|c|c|c|c|}
\hline & & 50 & & & & & & \\
\hline & & 39 & & & & & & \\
\hline & & 46 & & & & & & \\
\hline & & & & & & & & \\
\hline \multicolumn{9}{|l|}{$\begin{array}{l}\text { Waypoint } \\
86\end{array}$} \\
\hline $\begin{array}{l}37.48306 \\
119.20940\end{array}$ & 1395 & 42 & -90 & 2 & 15 & 1.00 & 201,65 & $r=6$ \\
\hline & & 52 & & 3 & 300 & 1.80 & 300 & \\
\hline & & 45 & & 1 & 30 & 2.00 & 354 & \\
\hline & & 52 & & 1.5 & 20 & 0.60 & $\begin{array}{l}18,66 \& \\
20,70\end{array}$ & \\
\hline & & 33 & & 0.3 & 10 & 0.80 & 280,60 & \\
\hline & & 51 & & 1.5 & face & 0.60 & $r=14$ & \\
\hline & & 32 & & 0.15 & face & 0.90 & & \\
\hline & & 38 & & & 75 & 1.10 & & \\
\hline & & 59 & & & 20 & 0.90 & & \\
\hline & & 48 & & & $r=4$ & $r=21$ & & \\
\hline & & 52 & & & & & & \\
\hline & & 61 & & & & & & \\
\hline & & 63 & & & & & & \\
\hline & & 49 & & & & & & \\
\hline & & 46 & & & & & & \\
\hline & & 48 & & & & & & \\
\hline & & $\overline{58}$ & & & & & & \\
\hline & & 49 & & & & & & \\
\hline & & & & & & & & \\
\hline $\begin{array}{l}\text { Waypoint } \\
87\end{array}$ & & & & & & & & \\
\hline $\begin{array}{l}37.48319 \\
119.20947\end{array}$ & 1399 & 47 & -90 & 8 & 200 & 1.80 & 184,79 & $r=5$ \\
\hline & & 58 & & 3 & 50 & 2.10 & 245,78 & \\
\hline & & 38 & & 1 & 25 & 1.20 & 325,77 & \\
\hline & & 61 & & 10 & 100 & 0.90 & $r=14$ & \\
\hline & & 46 & & 5.5 & 93.75 & 0.30 & & \\
\hline & & 60 & & 0.55 & 75 & 1.26 & & \\
\hline & & 37 & & & $r=2$ & 1.20 & & \\
\hline & & 46 & & & & $r=28$ & & \\
\hline & & 36 & & & & & & \\
\hline & & 49 & & & & & & \\
\hline
\end{tabular}




\begin{tabular}{|c|c|c|c|c|c|c|c|c|}
\hline & & 43 & & & & & & \\
\hline & & 36 & & & & & & \\
\hline & & 41 & & & & & & \\
\hline & & 36 & & & & & & \\
\hline & & 56 & & & & & & \\
\hline & & 44 & & & & & & \\
\hline & & 48 & & & & & & \\
\hline & & 46 & & & & & & \\
\hline & & & & & & & & \\
\hline $\begin{array}{l}\text { Waypoint } \\
88\end{array}$ & & & & & & & & \\
\hline $\begin{array}{l}37.48331 \\
119.21954\end{array}$ & 1398 & 59 & -90 & 10 & 180 & 0.25 & 270,56 & $r=5$ \\
\hline & & 62 & & 3.5 & 30 & 0.61 & 258 & \\
\hline & & 43 & & 2 & face(?) & 1.00 & 154,58 & \\
\hline & & 52 & & 2 & face(?) & 1.20 & 289 & \\
\hline & & 43 & & 2 & 30 & 0.50 & 196,71 & \\
\hline & & 53 & & 1 & 20 & 0.20 & 89,64 & \\
\hline & & 61 & & 1.5 & 20 & 0.30 & 78 & \\
\hline & & 67 & & 2 & $r=2$ & 1.50 & $r=14$ & \\
\hline & & 36 & & 0.2 & & 1.50 & & \\
\hline & & 55 & & & & 1.20 & & \\
\hline & & 53 & & & & 0.83 & & \\
\hline & & 63 & & & & 0.81 & & \\
\hline & & 48 & & & & $r=21$ & & \\
\hline & & 64 & & & & & & \\
\hline & & 45 & & & & & & \\
\hline & & 46 & & & & & & \\
\hline & & 51 & & & & & & \\
\hline & & 53 & & & & & & \\
\hline & & & & & & & & \\
\hline $\begin{array}{l}\text { Waypoint } \\
90\end{array}$ & & & & & & & & \\
\hline $\begin{array}{l}37.48277 \\
119.20753\end{array}$ & 1331 & 36 & -90 & 3 & 130 & 0.25 & 118,81 & $r=5$ \\
\hline & & 34 & & 1 & face & 0.90 & 180,47 & \\
\hline & & 28 & & 2.5 & 100 & 0.30 & NE block & \\
\hline & & 48 & & 4 & 20 & 0.30 & $r=14$ & \\
\hline & & 39 & & 1.5 & 50 & 0.60 & & \\
\hline & & 56 & & 2.5 & 60 & 1.50 & & \\
\hline
\end{tabular}




\begin{tabular}{|c|c|c|c|c|c|c|c|c|}
\hline & & 36 & & 0.25 & 150 & 1.20 & & \\
\hline & & 39 & & & $>20$ & 1.80 & & \\
\hline & & 33 & & & $r=2$ & 0.90 & & \\
\hline & & 43 & & & & 1.80 & & \\
\hline & & 50 & & & & 0.45 & & \\
\hline & & 50 & & & & 0.20 & & \\
\hline & & 54 & & & & 0.90 & & \\
\hline & & 40 & & & & 1.50 & & \\
\hline & & 33 & & & & 0.90 & & \\
\hline & & 37 & & & & 0.90 & & \\
\hline & & 34 & & & & $r=21$ & & \\
\hline & & 41 & & & & & & \\
\hline & & & & & & & & \\
\hline $\begin{array}{l}\text { Waypoint } \\
188\end{array}$ & & & & & & & & \\
\hline $\begin{array}{l}37.53322 \\
119.16740\end{array}$ & 1560 & 44 & -90 & 3 & 40 & 0.30 & 185,80 & $r=5$ \\
\hline & & 44 & & 3 & 50 & 0.30 & 194,70 & \\
\hline & & 52 & & 3 & 20 & 0.15 & 187,60 & \\
\hline & & 44 & & 2 & 30 & 0.20 & 259,60 & \\
\hline & & 52 & & 1.5 & 25 & 0.30 & 175 & \\
\hline & & 58 & & $\begin{array}{l}3.5 \\
\text { (Flake } \\
1 \text { ) }\end{array}$ & 25 & 0.50 & 239 & \\
\hline & & 54 & & 3 & $>20$ & 0.30 & $r=14$ & \\
\hline & & 61 & & 0.3 & $r=2$ & 0.40 & & \\
\hline & & 61 & & & & 1.00 & & \\
\hline & & 60 & & & & 0.90 & & \\
\hline & & 52 & & & & 0.15 & & \\
\hline & & 56 & & & & 0.20 & & \\
\hline & & \begin{tabular}{|l|}
50 \\
\end{tabular} & & & & 0.75 & & \\
\hline & & 49 & & & & 0.42 & & \\
\hline & & 57 & & & & 0.30 & & \\
\hline & & 60 & & & & $r=15$ & & \\
\hline & & 59 & & & & & & \\
\hline & & \begin{tabular}{|l|}
54 \\
\end{tabular} & & & & & & \\
\hline & & & & & & & & \\
\hline & & & & & & & & \\
\hline & & & & & & & & \\
\hline
\end{tabular}




\begin{tabular}{|c|c|c|c|c|c|c|c|c|}
\hline \multicolumn{9}{|l|}{$\begin{array}{l}\text { Waypoint } \\
189 \\
\end{array}$} \\
\hline $\begin{array}{l}37.53342 \\
119.16719\end{array}$ & 1580 & 60 & -90 & 3 & 1 & 0.40 & 250,65 & $r=5$ \\
\hline & & 57 & & 3.25 & 2 & 1.00 & 189,65 & \\
\hline & & 49 & & 1 & 2.5 & 1.50 & 141,69 & \\
\hline & & 60 & & 1 & 5 & 0.30 & 229,63 & \\
\hline & & 64 & & 3.3 & 2 & 0.15 & 145 & \\
\hline & & 49 & & 2.5 & 7.5 & 0.20 & 270,66 & \\
\hline & & 59 & & 2 & 5 & 0.40 & 131 & \\
\hline & & 55 & & 4 & 30.5 & 1.00 & 195,86 & \\
\hline & & 63 & & 1 & 4 & 1.20 & 146,55 & \\
\hline & & 62 & & 2.5 & 6.61 & 0.90 & $r=14$ & \\
\hline & & 64 & & 0.25 & 4 & 0.60 & & \\
\hline & & 50 & & & $r=5$ & 0.30 & & \\
\hline & & 52 & & & & 0.66 & & \\
\hline & & 54 & & & & 0.50 & & \\
\hline & & 58 & & & & $r=21$ & & \\
\hline & & 50 & & & & & & \\
\hline & & 51 & & & & & & \\
\hline & & 56 & & & & & & \\
\hline & & & & & & & & \\
\hline $\begin{array}{l}\text { Waypoint } \\
190\end{array}$ & & & & & & & & \\
\hline $\begin{array}{l}37.53392 \\
119.16698\end{array}$ & 1609 & 68 & -90 & 20 & 25 & 2.50 & 195 & $r=5$ \\
\hline & & 65 & & 1.5 & 50 & 1.50 & 239 & \\
\hline & & 66 & & 3 & 30 & 0.30 & 197 & \\
\hline & & 68 & & 3 & 75 & 1.00 & 134 & \\
\hline & & 68 & & 3.5 & 40 & 1.20 & 179,67 & \\
\hline & & 67 & & 1.5 & 30 & 2.00 & 130 & \\
\hline & & 67 & & 4 & 50 & 2.00 & 190,41 & \\
\hline & & 66 & & 1 & 30 & 3.50 & & \\
\hline & & 68 & & 20 & 41.25 & 0.90 & 193,59 & \\
\hline & & 67 & & 3.5 & 35 & 1.66 & 282,26 & \\
\hline & & 69 & & 1.5 & $r=2$ & 1.50 & 190,44 & \\
\hline & & 71 & & 3 & & $r=28$ & $r=9$ & \\
\hline & & 65 & & 0.3 & & & & \\
\hline & & 68 & & & & & & \\
\hline & & 67 & & & & & & \\
\hline
\end{tabular}




\begin{tabular}{|c|c|c|c|c|c|c|c|c|}
\hline & & 69 & & & & & & \\
\hline & & 62 & & & & & & \\
\hline & & 67 & & & & & & \\
\hline & & & & & & & & \\
\hline \multicolumn{9}{|l|}{$\begin{array}{l}\text { Waypoint } \\
191\end{array}$} \\
\hline $\begin{array}{l}37.53276 \\
119.16733\end{array}$ & 1544 & 48 & -90 & 4 & 50 & 1.50 & 195,85 & $r=5$ \\
\hline & & 50 & & 3 & 200 & 1.00 & 110 & \\
\hline & & 47 & & 10 & 200 & 0.30 & 218,56 & \\
\hline & & 50 & & 2 & 75 & 0.20 & 134 & \\
\hline & & 50 & & 1.5 & 25 & 0.20 & 190 & \\
\hline & & 54 & & 1 & 40 & 0.30 & 221 & \\
\hline & & 51 & & 2.5 & $>20$ & 0.15 & $r=9$ & \\
\hline & & 51 & & 0.25 & $r=2$ & 1.00 & & \\
\hline & & 48 & & & & 1.20 & & \\
\hline & & 50 & & & & 0.20 & & \\
\hline & & 44 & & & & 0.30 & & \\
\hline & & 47 & & & & 0.15 & & \\
\hline & & 46 & & & & 2.00 & & \\
\hline & & 53 & & & & 0.65 & & \\
\hline & & 52 & & & & 0 & & \\
\hline & & 45 & & & & $r=21$ & & \\
\hline & & 57 & & & & & & \\
\hline & & 5 & & & & & & \\
\hline & & & & & & & & \\
\hline $\begin{array}{l}\text { Waypoint } \\
192 \text { (SJ 7) }\end{array}$ & & & & & & & & \\
\hline $\begin{array}{l}37.53170 \\
119.17341\end{array}$ & 1506 & 73(P & -90 & 15 & 60 & 1.00 & 200,55 & $\mathrm{r}=6$ \\
\hline & & 72 & & 1.5 & $>20$ & 1.50 & $\mathrm{r}=9$ & \\
\hline & & \begin{tabular}{|l|}
74 \\
\end{tabular} & & & $r=2$ & 0.30 & & \\
\hline & & 71 & & & & 1.50 & & \\
\hline & & 75 & & & & 1.08 & & \\
\hline & & 68 & & & & 1.25 & & \\
\hline & & 73 & & & & $r=28$ & & \\
\hline & & 73 & & & & & & \\
\hline & & 72 & & & & & & \\
\hline & & \begin{tabular}{|l|}
71 \\
\end{tabular} & & & & & & \\
\hline & & 73 & & & & & & \\
\hline
\end{tabular}




\begin{tabular}{|c|c|c|c|c|c|c|c|}
\hline & & 70 & & & & & \\
\hline & & 74 & & & & & \\
\hline & & 74 & & & & & \\
\hline & & 72 & & & & & \\
\hline & & 70 & & & & & \\
\hline & & 70 & & & & & \\
\hline & & 72 & & & & & \\
\hline & & & & & & & \\
\hline & & 58 & & & & & \\
\hline & & 58 & & & & & \\
\hline & & 53 & & & & & \\
\hline & & 60 & & & & & \\
\hline & & 65 & & & & & \\
\hline & & 58 & & & & & \\
\hline & & 56 & & & & & \\
\hline & & 50 & & & & & \\
\hline & & 56 & & & & & \\
\hline & & 57 & & & & & \\
\hline & & 55 & & & & & \\
\hline & & 63 & & & & & \\
\hline & & 57 & & & & & \\
\hline & & 48 & & & & & \\
\hline & & 51 & & & & & \\
\hline & & 55 & & & & & \\
\hline & & 60 & & & & & \\
\hline & & 56 & & & & & \\
\hline & & & & & & & \\
\hline $\begin{array}{l}\text { Waypoint } \\
193\end{array}$ & & & & & & & \\
\hline $\begin{array}{l}37.53152 \\
119.17330\end{array}$ & 1513 & 60 (P-90 & 1 & 25 & 0.10 & 197,71 & $r=5$ \\
\hline & & 66 & 2 & 25 & 0.20 & 135 & \\
\hline & & 56 & 10 & 10 & 7.10 & 200,61 & \\
\hline & & 60 & 1.5 & 20 & 0.30 & $r=9$ & \\
\hline & & 56 & 1 & 30 & 1.00 & & \\
\hline & & 50 & 1.5 & 50 & 0.90 & & \\
\hline & & 62 & 0.15 & 40 & 0.50 & & \\
\hline & & 68 & & 12 & 1.00 & & \\
\hline & & 63 & & $>20$ & 0.60 & & \\
\hline & & 64 & & $r=4$ & 0.45 & & \\
\hline
\end{tabular}




\begin{tabular}{|c|c|c|c|c|c|c|c|c|}
\hline & & 60 & & & & 0.15 & & \\
\hline & & 62 & & & & 1.00 & & \\
\hline & & 66 & & & & 1.11 & & \\
\hline & & 64 & & & & 0.55 & & \\
\hline & & 68 & & & & $r=21$ & & \\
\hline & & 64 & & & & & & \\
\hline & & 63 & & & & & & \\
\hline & & 62 & & & & & & \\
\hline & & & & & & & & \\
\hline & & 72 & & & & & & \\
\hline & & 72 & & & & & & \\
\hline & & 65 & & & & & & \\
\hline & & 72 & & & & & & \\
\hline & & 71 & & & & & & \\
\hline & & 70 & & & & & & \\
\hline & & 70 & & & & & & \\
\hline & & 65 & & & & & & \\
\hline & & 72 & & & & & & \\
\hline & & 72 & & & & & & \\
\hline & & 72 & & & & & & \\
\hline & & 72 & & & & & & \\
\hline & & 62 & & & & & & \\
\hline & & 68 & & & & & & \\
\hline & & 64 & & & & & & \\
\hline & & 62 & & & & & & \\
\hline & & 68 & & & & & & \\
\hline & & 69 & & & & & & \\
\hline & & & & & & & & \\
\hline $\begin{array}{l}\text { Waypoint } \\
194\end{array}$ & & & & & & & & \\
\hline 37.53120 & & & & & & & & \\
\hline 119.17322 & 1517 & 64 & -90 & 10 & $30-75$ & 2.50 & 205,84 & $r=5$ \\
\hline & & 64 & & 2 & 30 & 2.00 & 187 & \\
\hline & & 64 & & 7 (F1) & 90 & 2.00 & 147 & \\
\hline & & 65 & & 20 & $>20$ & 2.40 & $r=14$ & \\
\hline & & 67 & & 10 & $r=2$ & 0.25 & & \\
\hline & & 66 & & 1 & & 0.10 & & \\
\hline & & 66 & & & & 0.20 & & \\
\hline & & 66 & & & & 0.30 & & \\
\hline & & 56 & & & & 3.00 & & \\
\hline
\end{tabular}




\begin{tabular}{|c|c|c|c|c|c|c|c|c|}
\hline & & 62 & & & & 1.42 & & \\
\hline & & 58 & & & & 2.00 & & \\
\hline & & 68 & & & & $r=28$ & & \\
\hline & & 70 & & & & & & \\
\hline & & 67 & & & & & & \\
\hline & & 68 & & & & & & \\
\hline & & 68 & & & & & & \\
\hline & & 68 & & & & & & \\
\hline & & 65 & & & & & & \\
\hline & & & & & & & & \\
\hline $\begin{array}{l}\text { Waypoint } \\
194 \mathrm{~A}\end{array}$ & & & & & & & & \\
\hline $\begin{array}{l}\text { No lat and } \\
\text { long. Next } \\
\text { step up from } \\
\text { waypoint } \\
194\end{array}$ & na & 63 & -90 & 3.5 & 50,70 & 1.20 & 189 & $r=6$ \\
\hline & & 65 & & 15 & 25 & 1.00 & $\overline{210,50}$ & \\
\hline & & 60 & & 1 & 25 & 10.00 & 175,86 & \\
\hline & & 56 & & 0.75 & 40 & 0.30 & 209,45 & \\
\hline & & 60 & & 3.5 & 5 & 0.20 & 286,27 & \\
\hline & & 65 & & 1 & 30 & 0.75 & 194 & \\
\hline & & 66 & & 30 & $>20$ & 1.00 & $r=14$ & \\
\hline & & 66 & & 3.5 & $r=2$ & 0.25 & & \\
\hline & & 65 & & 0.35 & & 0.50 & & \\
\hline & & 64 & & & & 0.15 & & \\
\hline & & 66 & & & & 1.00 & & \\
\hline & & 60 & & & & 2.00 & & \\
\hline & & 58 & & & & 1.53 & & \\
\hline & & 58 & & & & 0.88 & & \\
\hline & & 63 & & & & $r=21$ & & \\
\hline & & 58 & & & & & & \\
\hline & & 68 & & & & & & \\
\hline & & 62 & & & & & & \\
\hline & & & & & & & & \\
\hline & & & & & & & & \\
\hline & & & & & & & & \\
\hline & & & & & & & & \\
\hline
\end{tabular}




\begin{tabular}{|c|c|c|c|c|c|c|c|c|}
\hline \multicolumn{9}{|l|}{$\begin{array}{l}\text { Waypoint } \\
195\end{array}$} \\
\hline $\begin{array}{l}37.53037 \\
119.17336\end{array}$ & 1562 & 56 & -90 & $2(1 a)$ & 127 & 1.00 & 190,90 & $\mathrm{r}=5$ \\
\hline & & 64 & & $2.5(1 b)$ & 100 & 0.15 & 207,72 & \\
\hline & & 58 & & 0.3 & 75 & 0.15 & 240 & \\
\hline & & 57 & & 2 & 60 & 0.15 & 210 & \\
\hline & & 66 & & 7.5 & 30 & 0.30 & 184,70 & \\
\hline & & 63 & & 0.3 & 25 & 0.75 & 217,50 & \\
\hline & & 60 & & 1.15 & $>20$ & 0.40 & $\mathrm{r}=14$ & \\
\hline & & 55 & & 0.115 & $r=2$ & 0.41 & & \\
\hline & & 61 & & & & 0.30 & & \\
\hline & & 66 & & & & $r=15$ & & \\
\hline & & 56 & & & & & & \\
\hline & & 63 & & & & & & \\
\hline & & 62 & & & & & & \\
\hline & & 62 & & & & & & \\
\hline & & 64 & & & & & & \\
\hline & & 54 & & & & & & \\
\hline & & 60 & & & & & & \\
\hline & & 60 & & & & & & \\
\hline & & & & & & & & \\
\hline $\begin{array}{l}\text { Waypoint } \\
196 \text { (SJ6) }\end{array}$ & & & & & & & & \\
\hline $\begin{array}{l}37.51215 \\
119.19344\end{array}$ & 1412 & 52 & -90 & 0.5 & 15 & 0.30 & 199 & $r=5$ \\
\hline & & 50 & & 0.7 & 10 & 0.70 & 187 & \\
\hline & & 52 & & 1 & 35 & 0.50 & 128,45 & \\
\hline & & 56 & & 1 & 75 & 0.90 & 246 & \\
\hline & & 50 & & 0.7 & 25 & 0.90 & 234 & \\
\hline & & 57 & & 1.5 & 200 & 0.80 & 250,87 & \\
\hline & & 58 & & 2 & 130 & 0.80 & 187 & \\
\hline & & 52 & & 1.5 & 200 & 0.60 & 255,79 & \\
\hline & & 52 & & 1 & 230 & 0.20 & 129,56 & \\
\hline & & 50 & & 3.5 & 103 & 0.30 & 120,60 & \\
\hline & & 53 & & 3.5 & 130 & 0.60 & 268,79 & \\
\hline & & 55 & & 0.35 & $>20$ & 0.30 & $r=14$ & \\
\hline & & 53 & & & $r=2$ & 0.15 & & \\
\hline & & 53 & & & & 0.90 & & \\
\hline
\end{tabular}




\begin{tabular}{|c|c|c|c|c|c|c|c|c|}
\hline & & 52 & & & & 1.00 & & \\
\hline & & 57 & & & & 0.90 & & \\
\hline & & 50 & & & & 0.80 & & \\
\hline & & 53 & & & & 0.63 & & \\
\hline & & & & & & 0.70 & & \\
\hline & & & & & & $r=21$ & & \\
\hline $\begin{array}{l}\text { Waypoint } \\
197\end{array}$ & & & & & & & & \\
\hline $\begin{array}{l}37.51190 \\
119.19352\end{array}$ & 1423 & 52 & -90 & 0.8 & 10 & 1.20 & 200,74 & $r=5$ \\
\hline & & 54 & & 3.5 & 20 & 0.45 & 110 or 290 & \\
\hline & & 54 & & 1.5 & 180 & 0.15 & 260,80 & \\
\hline & & 50 & & 1 & 50 & 0.90 & 190,68 & \\
\hline & & 54 & & 2 & 25 & 0.25 & 165 & \\
\hline & & 58 & & 1 & 25 & 0.50 & 156,65 & \\
\hline & & 56 & & 2 & 30 & 0.90 & 164 & \\
\hline & & 52 & & 2 & 100 & 0.20 & 226,59 & \\
\hline & & 51 & & 3 & 150 & 0.80 & 189 & \\
\hline & & 55 & & 1.5 & 200 & 0.90 & 250,55 & \\
\hline & & 54 & & 3 & 10 & 0.30 & 110 & \\
\hline & & 56 & & 2.5 & 305 & 0.60 & 208 & \\
\hline & & 53 & & 1 & 50 & 0.20 & 259 & \\
\hline & & 54 & & 2 & 50 & 0.15 & 195 & \\
\hline & & 58 & & 1 & 15 & 0.60 & 219,76 & \\
\hline & & 45 & & 2 & $>20$ & 0.15 & $r=14$ & \\
\hline & & 55 & & 0.2 & $r=2$ & 0.20 & & \\
\hline & & 54 & & & & 0.30 & & \\
\hline & & & & & & 0.49 & & \\
\hline & & & & & & 0.38 & & \\
\hline & & & & & & $r=21$ & & \\
\hline $\begin{array}{l}\text { Waypoint } \\
198\end{array}$ & & & & & & & & \\
\hline $\begin{array}{l}37.51131 \\
119.19362\end{array}$ & 1451 & 60 & -90 & 10 & 1525 & $1-3 m$ & 201,39 & $r=6$ \\
\hline & & 62 & & 0.7 & 5 & $r=28$ & 143 & \\
\hline & & 66 & & 10 & 155 & & 223 & \\
\hline & & 62 & & 10 & 30 & & 195 & \\
\hline & & 58 & & 1.5 & 25 & & 238 & \\
\hline & & 55 & & 10 & 150 & & 205 & \\
\hline
\end{tabular}




\begin{tabular}{|c|c|c|c|c|c|c|c|c|}
\hline & & 60 & & 1.5 & 5 & & 137 & \\
\hline & & 55 & & 2 & 1 & & 245 & \\
\hline & & 68 & & 6 & 1 & & 255 & \\
\hline & & 60 & & 0.6 & 210.78 & & $r=14$ & \\
\hline & & 58 & & & 25 & & & \\
\hline & & 60 & & & $r=2$ & & & \\
\hline & & 52 & & & & & & \\
\hline & & 58 & & & & & & \\
\hline & & 60 & & & & & & \\
\hline & & 55 & & & & & & \\
\hline & & 59 & & & & & & \\
\hline & & & & & & & & \\
\hline $\begin{array}{l}\text { Waypoint } \\
199\end{array}$ & & & & & & & & \\
\hline $\begin{array}{l}37.51059 \\
119.19399\end{array}$ & 1476 & 62 & -90 & 4 & 5.3 & $1-3 m$ & 206 & $r=6$ \\
\hline & & 64 & & 1 & 25 & $r=28$ & 209 & \\
\hline & & 60 & & 0.6 & 5 & & 290,110 & \\
\hline & & 55 & & 4 & 10 & & 179 & \\
\hline & & 64 & & 3.5 & 25 & & 199 & \\
\hline & & 68 & & 3.5 & 1 & & $r=14$ & \\
\hline & & 64 & & 0.35 & 2 & & & \\
\hline & & 56 & & & 30 & & & \\
\hline & & 60 & & & 10 & & & \\
\hline & & 61 & & & 20 & & & \\
\hline & & 61 & & & 40 & & & \\
\hline & & 64 & & & 1 & & & \\
\hline & & 60 & & & 1 & & & \\
\hline & & 58 & & & 2 & & & \\
\hline & & 68 & & & 3 & & & \\
\hline & & 53 & & & 1 & & & \\
\hline & & 64 & & & 10 & & & \\
\hline & & 61 & & & 11.25 & & & \\
\hline & & & & & 5.3 & & & \\
\hline & & & & & $r=4$ & & & \\
\hline & & & & & & & & \\
\hline & & & & & & & & \\
\hline & & & & & & & & \\
\hline & & & & & & & & \\
\hline
\end{tabular}




\begin{tabular}{|c|c|c|c|c|c|c|c|c|}
\hline \multicolumn{9}{|l|}{$\begin{array}{l}\text { Waypoint } \\
200 \text { (SJ 5) }\end{array}$} \\
\hline \begin{tabular}{|l|}
37.51109 \\
119.19582
\end{tabular} & 1439 & $70(\mathrm{P}$ & -90 & 2 & 1 & 1.75 & 186 & $r=5$ \\
\hline & & 71 & & 2 & 25 & 3.00 & 155,36 & \\
\hline & & 72 & & 1 & face & 1.75 & 200 & \\
\hline & & 72 & & 0.5 & 100 & 0.60 & 146,43 & \\
\hline & & \begin{tabular}{|l|}
74 \\
\end{tabular} & & 1 & 115 & 0.50 & 276,73 & \\
\hline & & 74 & & 0.5 & 100 & 0.60 & 214 & \\
\hline & & 71 & & 1.5 & 100 & 0.30 & 185,90 & \\
\hline & & 73 & & 0.9 & 50 & 0.15 & 266 & \\
\hline & & 70 & & 1 & $r=2$ & 1.20 & $r=14$ & \\
\hline & & 72 & & 0.1 & & 0.30 & & \\
\hline & & 72 & & & & 0.60 & & \\
\hline & & 74 & & & & 0.90 & & \\
\hline & & 72 & & & & 0.60 & & \\
\hline & & 70 & & & & 0.90 & & \\
\hline & & 74 & & & & 0.94 & & \\
\hline & & 73 & & & & 0.60 & & \\
\hline & & & & & & $r=21$ & & \\
\hline & & 68 & & & & & & \\
\hline & & 72 & & & & & & \\
\hline & & 65 & & & & & & \\
\hline & & 70 & & & & & & \\
\hline & & 68 & & & & & & \\
\hline & & 66 & & & & & & \\
\hline & & 66 & & & & & & \\
\hline & & 70 & & & & & & \\
\hline & & 68 & & & & & & \\
\hline & & 63 & & & & & & \\
\hline & & 60 & & & & & & \\
\hline & & 64 & & & & & & \\
\hline & & 64 & & & & & & \\
\hline & & 67 & & & & & & \\
\hline & & 67 & & & & & & \\
\hline & & 68 & & & & & & \\
\hline & & 66 & & & & & & \\
\hline & & 67 & & & & & & \\
\hline & & & & & & & & \\
\hline
\end{tabular}




\begin{tabular}{|c|c|c|c|c|c|c|c|c|}
\hline $\begin{array}{l}\text { Waypoint } \\
201\end{array}$ & & & & & & & & \\
\hline 37.51139 & & & & & & & & \\
\hline 119.19610 & 1453 & 64 & -90 & 4 & 50 & 3.50 & 199 & $r=5$ \\
\hline & & 56 & & 2 & 15 & 1.00 & 225 & \\
\hline & & 63 & & 1.5 & 25 & 3.50 & 1149 & \\
\hline & & 70 & & 1.5 & 3 & 0.50 & 184 & \\
\hline & & 67 & & 3 & 50 & 1.20 & 285 & \\
\hline & & 57 & & 4.5 & 20 & 0.30 & 210 & \\
\hline & & 64 & & 2 & $\overline{10}$ & 0.60 & 195 & \\
\hline & & 52 & & 2 & 1 & 0.90 & 290 or 110 & \\
\hline & & 55 & & 1 & 12 & 1.20 & 130 & \\
\hline & & 59 & & 0.5 & 5 & 1.00 & 188 & \\
\hline & & 65 & & 3.5 & 7 & 1.50 & 216 & \\
\hline & & 55 & & 1 & 2 & 1.38 & 285 or 105 & \\
\hline & & 59 & & 2 & 6 & 1.00 & 283 & \\
\hline & & 57 & & 2 & $r=4$ & $r=21$ & $r=9$ & \\
\hline & & 70 & & 0.2 & & & & \\
\hline & & 52 & & & & & & \\
\hline & & 64 & & & & & & \\
\hline & & 61 & & & & & & \\
\hline & & & & & & & & \\
\hline $\begin{array}{l}\text { Waypoint } \\
202\end{array}$ & & & & & & & & \\
\hline $\begin{array}{l}37.51197 \\
119.19695\end{array}$ & 1502 & 58 & -90 & $\begin{array}{l}3.5(\mathrm{Set} \\
1)\end{array}$ & na & 0.30 & 194 & $r=5$ \\
\hline & & 54 & & $3(2)$ & 2 & 1.20 & 120 & \\
\hline & & 50 & & $1(3)$ & 150 & 0.45 & 145 & \\
\hline & & 61 & & $1.5(4)$ & 100 & 0.60 & 190 & \\
\hline & & 56 & & $3.5(5)$ & 2 & 1.50 & 166 & \\
\hline & & 58 & & $0.5(6)$ & na & 0.60 & 155,45 & \\
\hline & & 64 & & $2(7)$ & 100 & 0.30 & 225 & \\
\hline & & 56 & & $4(8)$ & 30 & 0.20 & 193 & \\
\hline & & 58 & & $3.5(9)$ & na & 1.80 & 229 & \\
\hline & & 42 & & $1(10)$ & 25 & 2.00 & 171 & \\
\hline & & 52 & & $>4(11)$ & 100 & 0.75 & 143 & \\
\hline & & 50 & & $>5(12)$ & face & 2.40 & 184,70 & \\
\hline & & 48 & & $3(13)$ & 25 & 0.60 & 279 & \\
\hline
\end{tabular}




\begin{tabular}{|c|c|c|c|c|c|c|c|c|}
\hline & & 55 & & & $r=2$ & 0.80 & $r=9$ & \\
\hline & & 57 & & & & 0.96 & & \\
\hline & & 46 & & & & 0.68 & & \\
\hline & & 56 & & & & $r=21$ & & \\
\hline & & 54 & & & & & & \\
\hline & & & & & & & & \\
\hline \multicolumn{9}{|l|}{$\begin{array}{l}\text { Waypoint } \\
203\end{array}$} \\
\hline $\begin{array}{l}37.51249 \\
119.19790\end{array}$ & 1524 & 52 & -90 & $>1.5(1)$ & 50 & 0.20 & 219 & $r=5$ \\
\hline & & 54 & & $0.7(2)$ & 75 & 0.30 & 265 & \\
\hline & & 57 & & $\begin{array}{l}1.6(\mathrm{Set} \\
3)\end{array}$ & 100 & 0.60 & 278 & \\
\hline & & 56 & & $2.5(4)$ & 50 & 0.30 & 232 & \\
\hline & & 57 & & $2(5)$ & 100 & 0.60 & 148 & \\
\hline & & 59 & & $1.2(6)$ & 50 & 0.50 & 161 & \\
\hline & & 55 & & $1.2(7)$ & 20 & 0.20 & 218 & \\
\hline & & 59 & & $2.5(8)$ & 150 & 0.75 & 171 & \\
\hline & & 59 & & $1(9)$ & 25 & 0.45 & 272 & \\
\hline & & 65 & & $1.5(10)$ & 10 & 0.45 & 133 & \\
\hline & & 60 & & $0.5(11)$ & 5 & 0.50 & 203 & \\
\hline & & 62 & & & \begin{tabular}{|l|l|}
57.73 \\
\end{tabular} & 0.60 & $r=9$ & \\
\hline & & 63 & & & 5 & 0.60 & & \\
\hline & & 60 & & & $r=2$ & 0.45 & & \\
\hline & & 64 & & & & 0.60 & & \\
\hline & & 60 & & & & 0.45 & & \\
\hline & & 65 & & & & 0.45 & & \\
\hline & & 5 & & & & 0.90 & & \\
\hline & & & & & & 1.75 & & \\
\hline & & & & & & 1.75 & & \\
\hline & & & & & & 1.75 & & \\
\hline & & & & & & 0.90 & & \\
\hline & & & & & & 1.60 & & \\
\hline & & & & & & 1.60 & & \\
\hline & & & & & & 0.20 & & \\
\hline & & & & & & 1.75 & & \\
\hline & & & & & & 1.60 & & \\
\hline & & & & & & 0.81 & & \\
\hline
\end{tabular}




\begin{tabular}{|l|l|l|l|l|l|l|l|l|}
\hline & & & & & & $\mathbf{0 . 6 0}$ & & \\
\hline & & & & & & $r=21$ & & \\
\hline
\end{tabular}




\begin{tabular}{|c|c|c|c|c|c|c|c|c|}
\hline \multicolumn{9}{|c|}{ North Fork King River valley } \\
\hline \multicolumn{9}{|l|}{$\begin{array}{l}\text { Waypoint } \\
128 \text { (K3) }\end{array}$} \\
\hline Lat/Long & $\begin{array}{l}\text { Elev. } \\
(\mathrm{m})\end{array}$ & $\mathrm{SH}$ & Alpha & \begin{tabular}{|l} 
Joint \\
length \\
$(\mathrm{m})$
\end{tabular} & \begin{tabular}{|l|} 
Joint \\
width \\
$(\mathrm{mm})$ \\
\end{tabular} & \begin{tabular}{|l|} 
Joint \\
strike and \\
dip
\end{tabular} & \begin{tabular}{|l|} 
Spacing \\
between \\
joints (m)
\end{tabular} & Continuity \\
\hline $\begin{array}{l}37.06516 \\
118.93773\end{array}$ & 2174 & 60 & -90 & no visible & $r=7$ & $r=20$ & $r=30$ & $r=7$ \\
\hline & & 50 & & & & & & \\
\hline & & 54 & & & & & & \\
\hline & & 60 & & & & & & \\
\hline & & 58 & & & & & & \\
\hline & & 62 & & & & & & \\
\hline & & 58 & & & & & & \\
\hline & & 40 & & & & & & \\
\hline & & 50 & & & & & & \\
\hline & & 60 & & & & & & \\
\hline & & 45 & & & & & & \\
\hline & & 50 & & & & & & \\
\hline & & 54 & & & & & & \\
\hline & & 55 & & & & & & \\
\hline & & 60 & & & & & & \\
\hline & & 62 & & & & & & \\
\hline & & 58 & & & & & & \\
\hline & & 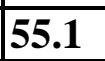 & & & & & & \\
\hline & & & & & & & & \\
\hline $\begin{array}{l}\text { Waypoint } \\
129\end{array}$ & & & & & & & & \\
\hline $\begin{array}{l}37.06528 \\
118.93782\end{array}$ & 2162 & $72(\mathrm{P})$ & -90 & $>5(1)$ & 25 & 220 & $1-3 m$ & $r=6$ \\
\hline & & 70 & & $>10(2)$ & 0 & 220,80 & $r=28$ & \\
\hline & & 70 & & & 152 & $r=18$ & & \\
\hline & & 70 & & & 10 & & & \\
\hline & & 66 & & & 46.75 & & & \\
\hline & & 69 & & & 17.5 & & & \\
\hline & & 64 & & & $r=4$ & & & \\
\hline & & 72 & & & & & & \\
\hline & & 70 & & & & & & \\
\hline & & 70 & & & & & & \\
\hline
\end{tabular}




\begin{tabular}{|l|l|l|l|l|l|l|l|l|}
\hline & & 68 & & & & & & \\
\hline & & 70 & & & & & & \\
\hline & & 68 & & & & & & \\
\hline & & 72 & & & & & & \\
\hline & & 68 & & & & & & \\
\hline & & 66 & & & & & & \\
\hline & & 70 & & & & & & \\
\hline & & $\mathbf{6 8 . 9}$ & & & & & & \\
\hline & & & & & & & & \\
\hline $\begin{array}{l}\text { Waypoint } \\
130\end{array}$ & & & & & & & & \\
\hline 37.06538 \\
118.93806
\end{tabular}




\begin{tabular}{|c|c|c|c|c|c|c|c|c|}
\hline & & 60 & & $0.5(8)$ & 1 & 285,80 & 0.7 & \\
\hline & & 64 & & $1(9)$ & 5 & 170 & 1.5 & \\
\hline & & 61 & & $2(10)$ & 10 & 30 & 1 & \\
\hline & & 60 & & $>4(11)$ & 25 & 40,76 & 1.2 & \\
\hline & & 63 & & & 44.82 & $r=9$ & 0.8 & \\
\hline & & 55 & & & 50 & & 0.8 & \\
\hline & & 62 & & & $r=2$ & & $r=21$ & \\
\hline & & 55 & & & & & & \\
\hline & & 52 & & & & & & \\
\hline & & 52 & & & & & & \\
\hline & & 57.8 & & & & & & \\
\hline & & & & & & & & \\
\hline $\begin{array}{l}\text { Waypoint } \\
132\end{array}$ & & & & & & & & \\
\hline $\begin{array}{l}37.06582 \\
118.9875\end{array}$ & 2113 & 57 & -90 & $>4(1)$ & 1 & 30,80 & 0.6 & $r=6$ \\
\hline & & 53 & & $>4(2)$ & 1 & 150 & 0.3 & \\
\hline & & 50 & & $3(3)$ & 3 & 185,90 & 1 & \\
\hline & & 50 & & $>4.5(4)$ & 2 & 180 & 0.2 & \\
\hline & & 50 & & $1.5(5)$ & 3 & $225,30(?)$ & 0.3 & \\
\hline & & 54 & & $1(\mathrm{~F} 1)$ & 5 & 45 & 0.2 & \\
\hline & & 55 & & & 1 & $r=14$ & 0.2 & \\
\hline & & $\sqrt[51]{2}$ & & & 1 & & 0.3 & \\
\hline & & $\overline{55}$ & & & 1 & & 0.25 & \\
\hline & & 52 & & & 50 & & 0.25 & \\
\hline & & 51 & & & 10 & & 0.6 & \\
\hline & & $\overline{58}$ & & & 5 & & 1.2 & \\
\hline & & 50 & & & 5 & & 1 & \\
\hline & & 56 & & & 2 & & 0.9 & \\
\hline & & 52 & & & 10 & & 0.52 & \\
\hline & & 52 & & & 5 & & 0.3 & \\
\hline & & 57 & & & 50 & & $r=21$ & \\
\hline & & 53.1 & & & 9.12 & & & \\
\hline & & & & & 3 & & & \\
\hline & & & & & & & & \\
\hline & & & & & & & & \\
\hline & & & & & & & & \\
\hline & & & & & & & & \\
\hline & & & & & & & & \\
\hline
\end{tabular}




\begin{tabular}{|c|c|c|c|c|c|c|c|c|}
\hline \multirow{2}{*}{$\begin{array}{l}\begin{array}{l}\text { Waypoint } \\
\mathbf{1 3 3}(\mathbf{K 2})\end{array} \\
37.05265 \\
118.94520\end{array}$} & & & & & $r=5$ & & & \\
\hline & 2116 & $70(\mathrm{P})$ & -90 & $>5(1)$ & 50 & 230,90 & 0.6 & $r=5$ \\
\hline & & 65 & & $>2(2)$ & 90 & 90,85 & 0.25 & \\
\hline & & 68 & & $1.5(3)$ & 2 & 190 & 1.2 & \\
\hline & & 66 & & $2(4)$ & 100 & 325,60 & 0.6 & \\
\hline & & 68 & & $2.5(5)$ & 150 & 30,60 & 1.8 & \\
\hline & & 68 & & $>5(\mathrm{~V} 1)$ & 150 & 315 & 0.3 & \\
\hline & & 68 & & $2(\mathrm{~V} 2)$ & 25 & 210 & 1.8 & \\
\hline & & 71 & & & 81 & $r=9$ & 0.94 & \\
\hline & & 66 & & & 90 & & 0.6 & \\
\hline & & 68 & & & $r=2$ & & $r=21$ & \\
\hline & & 64 & & & & & & \\
\hline & & 70 & & & & & & \\
\hline & & 68 & & & & & & \\
\hline & & 67 & & & & & & \\
\hline & & 63 & & & & & & \\
\hline & & 68 & & & & & & \\
\hline & & 69 & & & & & & \\
\hline & & 67.3 & & & & & & \\
\hline & & & & & & & & \\
\hline & & 62 & & & & & & \\
\hline & & 60 & & & & & & \\
\hline & & 66 & & & & & & \\
\hline & & 66 & & & & & & \\
\hline & & 56 & & & & & & \\
\hline & & 54 & & & & & & \\
\hline & & 56 & & & & & & \\
\hline & & 44 & & & & & & \\
\hline & & 50 & & & & & & \\
\hline & & 60 & & & & & & \\
\hline & & 52 & & & & & & \\
\hline & & 64 & & & & & & \\
\hline & & 51 & & & & & & \\
\hline & & 60 & & & & & & \\
\hline & & 61 & & & & & & \\
\hline & & 48 & & & & & & \\
\hline & & 53 & & & & & & \\
\hline & & 56.6 & & & & & & \\
\hline
\end{tabular}




\begin{tabular}{|c|c|c|c|c|c|c|c|c|}
\hline \multicolumn{9}{|l|}{$\begin{array}{l}\text { Waypoint } \\
134\end{array}$} \\
\hline $\begin{array}{l}37.05265 \\
118.94478\end{array}$ & 2099 & $68(\mathrm{P})$ & -90 & $>5(1)$ & 25 & 180 & 0.3 & $r=6$ \\
\hline & & 6 & & 5 & 0.1 & $r=18$ & 0.3 & \\
\hline & & 71 & & 0.5 & 2 & & 1.5 & \\
\hline & & 70 & & & 2 & & 1.8 & \\
\hline & & 70 & & & 0.01 & & 1.8 & \\
\hline & & 72 & & & 5.82 & & 1.14 & \\
\hline & & $\sqrt{69}$ & & & 2 & & 1.5 & \\
\hline & & 66 & & & $r=5$ & & $r=28$ & \\
\hline & & 74 & & & & & & \\
\hline & & 70 & & & & & & \\
\hline & & 66 & & & & & & \\
\hline & & 69 & & & & & & \\
\hline & & 69 & & & & & & \\
\hline & & 68 & & & & & & \\
\hline & & 68 & & & & & & \\
\hline & & 72 & & & & & & \\
\hline & & 72 & & & & & & \\
\hline & & 69.7 & & & & & & \\
\hline & & & & & & & & \\
\hline & & 61 & & & & & & \\
\hline & & 40 & & & & & & \\
\hline & & 45 & & & & & & \\
\hline & & 40 & & & & & & \\
\hline & & 60 & & & & & & \\
\hline & & 58 & & & & & & \\
\hline & & 54 & & & & & & \\
\hline & & 62 & & & & & & \\
\hline & & 52 & & & & & & \\
\hline & & 60 & & & & & & \\
\hline & & 44 & & & & & & \\
\hline & & 60 & & & & & & \\
\hline & & 56 & & & & & & \\
\hline & & 58 & & & & & & \\
\hline & & 62 & & & & & & \\
\hline & & 68 & & & & & & \\
\hline & & 54 & & & & & & \\
\hline & & \begin{tabular}{|l|}
54.9 \\
\end{tabular} & & & & & & \\
\hline
\end{tabular}




\begin{tabular}{|c|c|c|c|c|c|c|c|}
\hline \multicolumn{8}{|l|}{$\begin{array}{l}\text { Waypoint } \\
134 \mathrm{~A}\end{array}$} \\
\hline $\begin{array}{l}\text { No lat and } \\
\text { long. }\end{array}$ & $72(\mathrm{P})$ & -90 & $3(1)$ & 20 & 220 & 0.3 & $r=6$ \\
\hline & 71 & & $>3.5(2)$ & 10 & 335,20 & 1 & \\
\hline & 69 & & $1.5(3)$ & 10 & 170 & 0.6 & \\
\hline & 63 & & $2(4)$ & 5 & 170 & 0.3 & \\
\hline & 73 & & $1.5(5)$ & na & 290,60 & 1 & \\
\hline & 63 & & $2.5(6)$ & 10 & 290,65 & 2.5 & \\
\hline & 67 & & \begin{tabular}{|l|}
$0.5(7)$ \\
\end{tabular} & na & 170 & 1.2 & \\
\hline & 68 & & $3(8)$ & 30 & 290 & 0.5 & \\
\hline & 72 & & $3(9)$ & 15 & 225 & 0.25 & \\
\hline & 63 & & $3(10)$ & 40 & 55,85 & 0.8 & \\
\hline & 65 & & $4(11)$ & 100 & 10 & 1.8 & \\
\hline & 64 & & $1(12)$ & 2 & 220 & 1.4 & \\
\hline & 69 & & $>3(13)$ & 180 & 200,30 & 1.4 & \\
\hline & 62 & & $>3(14)$ & 100 & 200,30 & 0.2 & \\
\hline & 63 & & $>5(15)$ & 200 & 200,35 & 6 & \\
\hline & 64 & & & 55.54 & $r=18$ & 1.28 & \\
\hline & 64 & & & 20 & & 1 & \\
\hline & 66.3 & & & $r=5$ & & $r=28$ & \\
\hline & & & & & & & \\
\hline & 60 & -90 & & & & & \\
\hline & 63 & & & & & & \\
\hline & 54 & & & & & & \\
\hline & 57 & & & & & & \\
\hline & 58 & & & & & & \\
\hline & 56 & & & & & & \\
\hline & 51 & & & & & & \\
\hline & 49 & & & & & & \\
\hline & 61 & & & & & & \\
\hline & 52 & & & & & & \\
\hline & 44 & & & & & & \\
\hline & 60 & & & & & & \\
\hline & 70 & & & & & & \\
\hline & 58 & & & & & & \\
\hline & 54 & & & & & & \\
\hline & 54 & & & & & & \\
\hline & 52 & & & & & & \\
\hline & \begin{tabular}{|l|}
56.1 \\
\end{tabular} & & & & & & \\
\hline
\end{tabular}




\begin{tabular}{|c|c|c|c|c|c|c|c|c|}
\hline \multicolumn{9}{|l|}{$\begin{array}{l}\text { Waypoint } \\
135\end{array}$} \\
\hline $\begin{array}{l}37.05229 \\
118.94422\end{array}$ & 2070 & $70(\mathrm{P})$ & -90 & $>5(1)$ & 60 & 180,80 & 0.6 & $r=6$ \\
\hline & & 66 & & $>5(2)$ & 305 & 170 & 1.8 & \\
\hline & & 68 & & $2(3)$ & 75 & 260 & 3 & \\
\hline & & 58 & & $11(4)$ & 100 & 295 & 1 & \\
\hline & & 68 & & $3(5)$ & 75 & 265,82 & 1.2 & \\
\hline & & 70 & & $4(6)$ & 20 & 220,90 & 0.8 & \\
\hline & & $\overline{72}$ & & & 10 & $r=18$ & 1 & \\
\hline & & 70 & & & 30 & & 0.15 & \\
\hline & & 65 & & & 84.38 & & 0.3 & \\
\hline & & 72 & & & \begin{tabular}{|l|}
67.5 \\
\end{tabular} & & 1.09 & \\
\hline & & 60 & & & $r=2$ & & 1 & \\
\hline & & 70 & & & & & $r=21$ & \\
\hline & & 72 & & & & & & \\
\hline & & 65 & & & & & & \\
\hline & & 72 & & & & & & \\
\hline & & 60 & & & & & & \\
\hline & & 72 & & & & & & \\
\hline & & 67.5 & & & & & & \\
\hline & & & & & & & & \\
\hline & & 60 & & & & & & \\
\hline & & 62 & & & & & & \\
\hline & & 50 & & & & & & \\
\hline & & 52 & & & & & & \\
\hline & & 62 & & & & & & \\
\hline & & 56 & & & & & & \\
\hline & & 52 & & & & & & \\
\hline & & 56 & & & & & & \\
\hline & & 62 & & & & & & \\
\hline & & 58 & & & & & & \\
\hline & & 60 & & & & & & \\
\hline & & 51 & & & & & & \\
\hline & & 60 & & & & & & \\
\hline & & 48 & & & & & & \\
\hline & & 55 & & & & & & \\
\hline & & 65 & & & & & & \\
\hline & & 55 & & & & & & \\
\hline & & 56 & & & & & & \\
\hline
\end{tabular}




\begin{tabular}{|c|c|c|c|c|c|c|c|c|}
\hline \multirow{2}{*}{\begin{tabular}{|l|}
$\begin{array}{l}\text { Waypoint } \\
\mathbf{1 3 6}(\mathbf{K 1})\end{array}$ \\
37.05656 \\
118.94321
\end{tabular}} & & & & & & & & \\
\hline & 2058 & $72(\mathrm{P})$ & -90 & $>10(1)$ & 1 & 325 & 1.2 & $r=6$ \\
\hline & & 68 & & $>10(2)$ & 2 & 355 & 1.5 & \\
\hline & & 70 & & $4(3)$ & 1 & 235,85 & 1.8 & \\
\hline & & 72 & & & 3 & $r=20$ & 2.5 & \\
\hline & & 74 & & & 1 & & 3 & \\
\hline & & 72 & & & 1.6 & & 2 & \\
\hline & & 75 & & & 1 & & 1.8 & \\
\hline & & 71 & & & $r=6$ & & $r=28$ & \\
\hline & & 72 & & & & & & \\
\hline & & 69 & & & & & & \\
\hline & & 72 & & & & & & \\
\hline & & 70 & & & & & & \\
\hline & & 68 & & & & & & \\
\hline & & 66 & & & & & & \\
\hline & & 72 & & & & & & \\
\hline & & 71 & & & & & & \\
\hline & & 69 & & & & & & \\
\hline & & 70.7 & & & & & & \\
\hline & & & & & & & & \\
\hline & & 54 & & & & & & \\
\hline & & 44 & & & & & & \\
\hline & & 60 & & & & & & \\
\hline & & 52 & & & & & & \\
\hline & & 50 & & & & & & \\
\hline & & 56 & & & & & & \\
\hline & & 40 & & & & & & \\
\hline & & 54 & & & & & & \\
\hline & & 55 & & & & & & \\
\hline & & 48 & & & & & & \\
\hline & & 56 & & & & & & \\
\hline & & 52 & & & & & & \\
\hline & & 45 & & & & & & \\
\hline & & 52 & & & & & & \\
\hline & & 47 & & & & & & \\
\hline & & 58 & & & & & & \\
\hline & & 61 & & & & & & \\
\hline & & 52 & & & & & & \\
\hline
\end{tabular}




\begin{tabular}{|c|c|c|c|c|c|c|c|c|}
\hline \multicolumn{9}{|l|}{$\begin{array}{l}\text { Waypoint } \\
137\end{array}$} \\
\hline $\begin{array}{l}37.05655 \\
118.94260\end{array}$ & 2065 & $70(\mathrm{P}$ & -90 & $2-2.5$ & 50 & 35 (as flak & 0.3 & $r=5$ \\
\hline & & 74 & & $>10(2)$ & $\overline{100}$ & 325,90 & 0.5 & \\
\hline & & 68 & & $>7(3)$ & 90 & 45,60 & 0.25 & \\
\hline & & 70 & & $1.5(4)$ & $\overline{10}$ & 145,80 & 0.25 & \\
\hline & & 72 & & & $r=2$ & $r=9$ & 0.5 & \\
\hline & & 72 & & & & & 0.3 & \\
\hline & & $\overline{70}$ & & & & & 0.45 & \\
\hline & & 69 & & & & & 0.2 & \\
\hline & & 66 & & & & & 0.6 & \\
\hline & & 70 & & & & & 1.8 & \\
\hline & & 72 & & & & & 1 & \\
\hline & & 68 & & & & & 1 & \\
\hline & & 72 & & & & & 1.2 & \\
\hline & & 70 & & & & & 1 & \\
\hline & & 70 & & & & & 1.2 & \\
\hline & & 64 & & & & & 1 & \\
\hline & & 71 & & & & & 1 & \\
\hline & & 69.9 & & & & & 1.2 & \\
\hline & & 50 & & & & & 2.5 & \\
\hline & & $\overline{52}$ & & & & & 0.86 & \\
\hline & & 48 & & & & & 1 & \\
\hline & & 56 & & & & & $r=21$ & \\
\hline & & 60 & & & & & & \\
\hline & & 58 & & & & & & \\
\hline & & 60 & & & & & & \\
\hline & & 52 & & & & & & \\
\hline & & 54 & & & & & & \\
\hline & & 42 & & & & & & \\
\hline & & 59 & & & & & & \\
\hline & & 52 & & & & & & \\
\hline & & 61 & & & & & & \\
\hline & & 43 & & & & & & \\
\hline & & 60 & & & & & & \\
\hline & & 62 & & & & & & \\
\hline & & 60 & & & & & & \\
\hline & & 54.6 & & & & & & \\
\hline & & & & & & & & \\
\hline
\end{tabular}




\begin{tabular}{|c|c|c|c|c|c|c|c|c|}
\hline \multicolumn{9}{|l|}{$\begin{array}{l}\text { Waypoint } \\
138\end{array}$} \\
\hline $\begin{array}{l}37.05662 \\
118.94205\end{array}$ & 2083 & $71(\mathrm{P})$ & -90 & $1.5(1)$ & 20 & 330,82 & $r=30$ & $r=6$ \\
\hline & & 66 & & $2(2)$ & 10 & 145 & & \\
\hline & & 68 & & $2.5(3)$ & $\overline{75}$ & 25,85 & & \\
\hline & & 70 & & $3.5(4)$ & 30 & 55,90 & & \\
\hline & & \begin{tabular}{|l|}
71 \\
\end{tabular} & & & $r=6$ & $r=9$ & & \\
\hline & & 68 & & & & & & \\
\hline & & 72 & & & & & & \\
\hline & & 68 & & & & & & \\
\hline & & 70 & & & & & & \\
\hline & & 73 & & & & & & \\
\hline & & 70 & & & & & & \\
\hline & & 69 & & & & & & \\
\hline & & 72 & & & & & & \\
\hline & & 70 & & & & & & \\
\hline & & 72 & & & & & & \\
\hline & & 71 & & & & & & \\
\hline & & 68 & & & & & & \\
\hline & & 69.9 & & & & & & \\
\hline & & & & & & & & \\
\hline $\begin{array}{l}\text { Waypoint } \\
139\end{array}$ & & & & & & & & \\
\hline $\begin{array}{l}37.05666 \\
118.94156\end{array}$ & 2097 & 74 & -45 & $15(1)$ & 10 & 350,90 & 0.9 & $r=6$ \\
\hline & & 74 & & $10(2)$ & na & 55 & 0.6 & \\
\hline & & 71 & & $3.5(\mathrm{~F} 1)$ & 1 & 150 & 1.8 & \\
\hline & & 70 & & $4(\mathrm{~F} 2)$ & 0.1 & 140 & 1.5 & \\
\hline & & 70 & & $>10(\mathrm{~V} 1)$ & 2 & 155 & 2.1 & \\
\hline & & 69 & & & 1.5 & $r=14$ & 1.38 & \\
\hline & & \begin{tabular}{|l|}
72 \\
\end{tabular} & & & $r=5$ & & 1.5 & \\
\hline & & 71 & & & & & $r=28$ & \\
\hline & & 70 & & & & & & \\
\hline & & 67 & & & & & & \\
\hline & & 70 & & & & & & \\
\hline & & 72 & & & & & & \\
\hline & & 72 & & & & & & \\
\hline & & 71 & & & & & & \\
\hline & & 71 & & & & & & \\
\hline
\end{tabular}




\begin{tabular}{|c|c|c|c|c|c|c|c|c|}
\hline & & 69 & & & & & & \\
\hline & & 70.8 & & & & & & \\
\hline & & & & & & & & \\
\hline \multicolumn{9}{|l|}{$\begin{array}{l}\text { Waypoint } \\
140\end{array}$} \\
\hline \multirow[t]{22}{*}{$\begin{array}{l}37.05666 \\
118.94155 \\
\text { (very poor } \\
\text { satellite } \\
\text { reception.) }\end{array}$} & 2212 & 61 & -45 & $2-2.5$ & 50 & 50,30 & 0.6 & $r-6$ \\
\hline & & 58 & & $2(2)$ & 1 & 350 & 0.25 & \\
\hline & & 56 & & $0.5-1.5$ & 1 & 335,80 & 0.2 & \\
\hline & & 52 & & & 2 & $r=14$ & 0.15 & \\
\hline & & 43 & & & 1 & & 0.15 & \\
\hline & & 58 & & & 4 & & 0.25 & \\
\hline & & 60 & & & 2 & & 0.2 & \\
\hline & & 44 & & & 3 & & 0.15 & \\
\hline & & 61 & & & 2 & & 0.9 & \\
\hline & & 47 & & & 100 & & 0.8 & \\
\hline & & 60 & & & 51 & & 0.15 & \\
\hline & & 48 & & & 25 & & \begin{tabular}{|l|}
0.6 \\
\end{tabular} & \\
\hline & & 61 & & & 50 & & 0.3 & \\
\hline & & 56 & & & 10 & & \begin{tabular}{|l|}
0.36 \\
\end{tabular} & \\
\hline & & 50 & & & 150 & & 0.25 & \\
\hline & & 52 & & & 10 & & $r=15$ & \\
\hline & & 61 & & & 20 & & & \\
\hline & & 54.6 & & & 200 & & & \\
\hline & & & & & 100 & & & \\
\hline & & & & & 41.16 & & & \\
\hline & & & & & 10 & & & \\
\hline & & & & & $r=4$ & & & \\
\hline \multicolumn{9}{|l|}{$\begin{array}{l}\text { Waypoint } \\
141(\mathrm{~K} 5)\end{array}$} \\
\hline $\begin{array}{l}37.08646 \\
118.89583\end{array}$ & 2379 & 52 & -90 & $>4$ (1) & 75 & 175,70 & 0.5 & $r=5$ \\
\hline & & 60 & & $>4(2)$ & 90 & 220 & 1 & \\
\hline & & 61 & & $1.75(3)$ & 1 & 145 & \begin{tabular}{|l|}
0.15 \\
\end{tabular} & \\
\hline & & 50 & & \begin{tabular}{|l}
$1(4)$ \\
\end{tabular} & 5 & 150,60 & 0.9 & \\
\hline & & 54 & & $1(5)$ & 1 & 290 & 1.8 & \\
\hline & & 52 & & $2(6)$ & 2 & 220,50 & 1 & \\
\hline
\end{tabular}




\begin{tabular}{|c|c|c|c|c|c|c|c|c|}
\hline & & 50 & & & 29 & $r=9$ & 0.6 & \\
\hline & & 56 & & & 3.5 & & 0.15 & \\
\hline & & 50 & & & $r=6$ & & 1 & \\
\hline & & 50 & & & & & 1.5 & \\
\hline & & 58 & & & & & 0.86 & \\
\hline & & 52 & & & & & 0.95 & \\
\hline & & 58 & & & & & $r=21$ & \\
\hline & & 63 & & & & & & \\
\hline & & 56 & & & & & & \\
\hline & & 58 & & & & & & \\
\hline & & 59 & & & & & & \\
\hline & & 55.2 & & & & & & \\
\hline & & & & & & & & \\
\hline $\begin{array}{l}\text { Waypoint } \\
142\end{array}$ & & & & & & & & \\
\hline $\begin{array}{l}37.08659 \\
118.89581\end{array}$ & 2374 & 55 & -45 & $>20(1)$ & 150 & 210,85 & 1.8 & $r=5$ \\
\hline & & 60 & & $1(2)$ & 10 & 260,90 & 2.4 & \\
\hline & & 58 & & $2.5(3)$ & na & 305 & 2.75 & \\
\hline & & 56 & & $3(4)$ & 0 & 200 & 0.3 & \\
\hline & & 57 & & $4.5(\mathrm{~F} 1)$ & 75 & 115 & 0.6 & \\
\hline & & 50 & & $3(\mathrm{~F} 2)$ & 15 & 120 & 0.15 & \\
\hline & & 58 & & $>15$ (V1) & 305 & 195 & 2.4 & \\
\hline & & 49 & & $>4(\mathrm{~V} 2)$ & 50 & 125 & 0.2 & \\
\hline & & 41 & & $>10(\mathrm{~V} 3)$ & 40 & 120 & 0.15 & \\
\hline & & 61 & & & 1 & $r=14$ & 0.6 & \\
\hline & & 60 & & & 1 & & 0.3 & \\
\hline & & 53 & & & 2 & & 0.6 & \\
\hline & & 60 & & & 10 & & 1 & \\
\hline & & 42 & & & 20 & & 1.02 & \\
\hline & & 59 & & & 50 & & 0.6 & \\
\hline & & 54 & & & 1 & & $r=21$ & \\
\hline & & 58 & & & 48.67 & & & \\
\hline & & 54.8 & & & 15 & & & \\
\hline & & & & & $r=4$ & & & \\
\hline & & & & & & & & \\
\hline & & & & & & & & \\
\hline & & & & & & & & \\
\hline & & & & & & & & \\
\hline & & & & & & & & \\
\hline
\end{tabular}




\begin{tabular}{|c|c|c|c|c|c|c|c|c|}
\hline \multicolumn{9}{|l|}{\begin{tabular}{|l|} 
Waypoint \\
143
\end{tabular}} \\
\hline $\begin{array}{l}37.08673 \\
118.89556\end{array}$ & 2360 & 53 & -45 & $3(1)$ & 4 & 190 & 0.75 & $r=6$ \\
\hline & & 58 & & $3(2)$ & face & 70,60 & 0.3 & \\
\hline & & 61 & & $3.5(\mathrm{~F} 3)$ & 120 & 150 & 0.15 & \\
\hline & & 48 & & $2(\mathrm{~F} 4)$ & 50 & 205 & 0.15 & \\
\hline & & 56 & & $2(5)$ & face & 210,80 & 0.45 & \\
\hline & & 60 & & $2(\mathrm{~F} 6)$ & 50 & 275 & 0.45 & \\
\hline & & 44 & & $2.5(7)$ & face & 200 & 0.5 & \\
\hline & & 52 & & $>3(8)$ & 75 & $r=9$ & 0.2 & \\
\hline & & 58 & & & $r=2$ & & 0.3 & \\
\hline & & 61 & & & & & 0.15 & \\
\hline & & 54 & & & & & 0.3 & \\
\hline & & 47 & & & & & 0.3 & \\
\hline & & 56 & & & & & 0.15 & \\
\hline & & 58 & & & & & 0.25 & \\
\hline & & 46 & & & & & 0.2 & \\
\hline & & 52 & & & & & 0.3 & \\
\hline & & 54 & & & & & 0.4 & \\
\hline & & 54 & & & & & 0.25 & \\
\hline & & & & & & & 0.31 & \\
\hline & & & & & & & 0.3 & \\
\hline & & & & & & & $r=15$ & \\
\hline & & & & & & & & \\
\hline $\begin{array}{l}\text { Waypoint } \\
144\end{array}$ & & & & & & & & \\
\hline $\begin{array}{l}37.08733 \\
118.89536\end{array}$ & 2370 & 48 & -90 & $1.5(1)$ & 150 & 0,20 & 1.2 & $r-5$ \\
\hline & & 56 & & $>4(2)$ & face & 95,80 & 0.3 & \\
\hline & & 60 & & $>4.5(3)$ & 75 & 15,55 & 0.45 & \\
\hline & & 49 & & $3.5(4)$ & 10 & 115 & 1.2 & \\
\hline & & 50 & & $>3(5)$ & 10 & 40 & 1.5 & \\
\hline & & 58 & & $1(6)$ & 30 & 35 & 1 & \\
\hline & & 56 & & 1 (7) & 10 & 60 & 1 & \\
\hline & & 52 & & $>2(8)$ & 0 & 140 & 1.2 & \\
\hline & & 53 & & $3(9)$ & 0 & 20 & 1.8 & \\
\hline & & 55 & & & \begin{tabular}{|l|l|}
35.63 \\
\end{tabular} & $r=9$ & 1.07 & \\
\hline & & 57 & & & 10 & & 1.2 & \\
\hline & & 58 & & & $r=4$ & & $r=28$ & \\
\hline
\end{tabular}




\begin{tabular}{|c|c|c|c|c|c|c|c|c|}
\hline & & 55 & & & & & & \\
\hline & & 60 & & & & & & \\
\hline & & 52 & & & & & & \\
\hline & & 44 & & & & & & \\
\hline & & 55 & & & & & & \\
\hline & & 54 & & & & & & \\
\hline & & & & & & & & \\
\hline & & & & & & & & \\
\hline $\begin{array}{l}\text { Waypoint } \\
145\end{array}$ & & & & & & & & \\
\hline $\begin{array}{l}37.08774 \\
118.89510\end{array}$ & 2388 & 55 & -90 & $>10(1)$ & 25 & 45,75 & 0.3 & $r=5$ \\
\hline & & 50 & & $4(2)$ & 0.001 & 25 & 1.8 & \\
\hline & & 57 & & $1(3)$ & 0.001 & $65(\mathrm{~J}), 160$ & 2 & \\
\hline & & 43 & & $3(\mathrm{~F} 4)$ & 20 & 285 & 0.3 & \\
\hline & & 54 & & $0.5-2.5(\mathrm{~S}$ & 50 & 135,55 & 0.6 & \\
\hline & & 57 & & $>5(6)$ & 130 & 30,80 & 0.45 & \\
\hline & & 42 & & $1.5(7)$ & 2 & 0,90 & 0.45 & \\
\hline & & 61 & & 0.3 (F8) & 100 & 55 & 1.2 & \\
\hline & & 53 & & $2(9)$ & 5 & 90 & 1 & \\
\hline & & 45 & & & 10 & $r=9$ & 0.6 & \\
\hline & & 60 & & & 60 & & 0.45 & \\
\hline & & 53 & & & 40 & & 1 & \\
\hline & & 58 & & & 20 & & 1.2 & \\
\hline & & 60 & & & 30 & & 0.3 & \\
\hline & & 48 & & & 100 & & 0.3 & \\
\hline & & 62 & & & 20 & & 0.45 & \\
\hline & & 53.6 & & & 10 & & 3 & \\
\hline & & & & & 36.59 & & 0.91 & \\
\hline & & & & & 20 & & 0.6 & \\
\hline & & & & & $r=4$ & & $r=21$ & \\
\hline & & & & & & & & \\
\hline $\begin{array}{l}\text { Waypoint } \\
146\end{array}$ & & & & & & & & \\
\hline $\begin{array}{l}37.08809 \\
118.89482\end{array}$ & 2411 & 50 & -90 & 5.5 (Set 1 & 130 & 355 & $1-3 m$ & $r=5$ \\
\hline & & 40 & & $1.75-3.5$ & 200 & $70,20(?)$ & $r=28$ & \\
\hline & & 59 & & $2(3)$ & 40 & 100 & & \\
\hline & & 58 & & $3(4)$ & 30 & 350 & & \\
\hline & & 60 & & $2(5)$ & 130 & 225,75 & & \\
\hline
\end{tabular}




\begin{tabular}{|c|c|c|c|c|c|c|c|c|}
\hline & & 42 & & $>3(6)$ & 100 & 325 & & \\
\hline & & 60 & & & 105 & $r=14$ & & \\
\hline & & 58 & & & 115 & & & \\
\hline & & 52 & & & $r=2$ & & & \\
\hline & & 60 & & & & & & \\
\hline & & 54 & & & & & & \\
\hline & & 61 & & & & & & \\
\hline & & 56 & & & & & & \\
\hline & & 50 & & & & & & \\
\hline & & 52 & & & & & & \\
\hline & & 60 & & & & & & \\
\hline & & 58 & & & & & & \\
\hline & & 54.7 & & & & & & \\
\hline & & & & & & & & \\
\hline $\begin{array}{l}\text { Waypoint } \\
147 \text { (K4) }\end{array}$ & & & & & & & & \\
\hline $\begin{array}{l}37.07589 \\
118.91648 \\
\end{array}$ & 2196 & 60 & -90 & $>5(1)$ & 100 & 20 & $1-3 m$ & $r=6$ \\
\hline & & 51 & & $>4(2)$ & 100 & 180,75 & $r=28$ & \\
\hline & & 42 & & $1(3)$ & na & 320 & & \\
\hline & & 54 & & $>5(4)$ & 150 & 10,70 & & \\
\hline & & 50 & & $3(5)$ & na & 355,90 & & \\
\hline & & 40 & & $3(?)(\mathrm{F} 1)$ & 0.001 & 115 & & \\
\hline & & 50 & & & 1 & $r=18$ & & \\
\hline & & 60 & & & 1 & & & \\
\hline & & 56 & & & 2 & & & \\
\hline & & 55 & & & 0.001 & & & \\
\hline & & 61 & & & 44.25 & & & \\
\hline & & 42 & & & 1.5 & & & \\
\hline & & 58 & & & $r=5$ & & & \\
\hline & & 50 & & & & & & \\
\hline & & 58 & & & & & & \\
\hline & & 54 & & & & & & \\
\hline & & 60 & & & & & & \\
\hline & & 53 & & & & & & \\
\hline & & & & & & & & \\
\hline & & & & & & & & \\
\hline & & & & & & & & \\
\hline & & & & & & & & \\
\hline
\end{tabular}




\begin{tabular}{|c|c|c|c|c|c|c|c|c|}
\hline \multicolumn{9}{|l|}{$\begin{array}{l}\text { Waypoint } \\
147\end{array}$} \\
\hline $\begin{array}{l}37.07623 \\
118.91647\end{array}$ & 2211 & 40 & -90 & $4-10($ Set $)$ & 75 & 10,90 & 1 & $r=5$ \\
\hline & & 57 & & 4 (Set 2) & 10 & 290,80 & 1.2 & \\
\hline & & 51 & & $3(3)$ & 60 & 280 & 1.5 & \\
\hline & & 59 & & $2.5(4)$ & 75 & 255,86 & 1.2 & \\
\hline & & 46 & & $2(5)$ & na & 350 & 1.8 & \\
\hline & & 57 & & $3.5(6)$ & 125 & 85,70 & 3 & \\
\hline & & 53 & & $1.5(\mathrm{~F} 1)$ & 25 & 15 & 0.6 & \\
\hline & & 60 & & $1(\mathrm{~F} 2)$ & 10 & 280,77 & 1 & \\
\hline & & 54 & & & $r=2$ & $r=14$ & 0.9 & \\
\hline & & 48 & & & & & 2.5 & \\
\hline & & 52 & & & & & 0.6 & \\
\hline & & 55 & & & & & 2.5 & \\
\hline & & 56 & & & & & 1 & \\
\hline & & 62 & & & & & 0.9 & \\
\hline & & 54 & & & & & 1.41 & \\
\hline & & 47 & & & & & 1.1 & \\
\hline & & 56 & & & & & $r=28$ & \\
\hline & & 53.4 & & & & & & \\
\hline & & & & & & & & \\
\hline $\begin{array}{l}\text { Waypoint } \\
149\end{array}$ & & & & & & & & \\
\hline $\begin{array}{l}37.07677 \\
118.91641\end{array}$ & 2227 & 63 & -45 & $3(1)$ & 200 & 80,86 & 0.3 & $r=5$ \\
\hline & & 54 & & $>66(2)$ & 300 & 30,90 & 0.45 & \\
\hline & & 58 & & $1.5(3)$ & 120 & 5,85 & 0.6 & \\
\hline & & 50 & & $1.5(4)$ & 100 & 35,90 & 0.3 & \\
\hline & & 57 & & $>5(5)$ & 60 & 355,90 & 0.2 & \\
\hline & & 46 & & \begin{tabular}{|l|}
$4(6)$ \\
\end{tabular} & na & 105 & 0.3 & \\
\hline & & 52 & & 0.3 (Set 1 & $r=2$ & $r=14$ & 0.9 & \\
\hline & & 56 & & $0.2($ Set 2$)$ & & & 0.75 & \\
\hline & & 58 & & & & & 1.2 & \\
\hline & & 54 & & & & & 1.2 & \\
\hline & & 43 & & & & & 1.5 & \\
\hline & & 54 & & & & & 2 & \\
\hline & & 58 & & & & & 2 & \\
\hline & & 48 & & & & & 1 & \\
\hline & & 62 & & & & & 0.9 & \\
\hline
\end{tabular}




\begin{tabular}{|l|l|l|l|l|l|l|l|l|}
\hline & & 56 & & & & & 1 & \\
\hline & & 61 & & & & & 1.2 & \\
\hline & & $\mathbf{5 4 . 7}$ & & & & & $\mathbf{0 . 9 3}$ & \\
\hline & & & & & & & $\mathbf{0 . 9}$ & \\
\hline & & & & & & & $\boldsymbol{r}=\mathbf{2 1}$ & \\
\hline $\begin{array}{l}\text { Waypoint } \\
150\end{array}$ & & & & & & & & \\
\hline $\begin{array}{l}37.07800 \\
118.91647\end{array}$ & 2269 & 57 & -90 & $8(1)$ & 0.5 & 5 & $>3 \mathrm{~m}$ & $\boldsymbol{r}=\mathbf{6}$ \\
\hline & & 46 & & $3(2)$ & 0.001 & 5 & $\boldsymbol{r}=\mathbf{3 0}$ & \\
\hline & & 63 & & $5(3)$ & 1 & 45,90 & & \\
\hline & & 60 & & $4(\mathrm{~F} 1)$ & 1 & 20 & & \\
\hline & & 53 & & $2.5(\mathrm{~F} 2)$ & 1 & 40 & & \\
\hline & & 44 & & & 0.1 & $\boldsymbol{r}=\mathbf{9}$ & & \\
\hline & & 54 & & & 0.5 & & & \\
\hline & & 62 & & & $\mathbf{0 . 5 9}$ & & & \\
\hline & & 52 & & & $\mathbf{0 . 5}$ & & & \\
\hline & & 55 & & & $\boldsymbol{r}=\mathbf{6}$ & & & \\
\hline & & 60 & & & & & & \\
\hline & & 50 & & & & & & \\
\hline & & 43 & & & & & & \\
\hline & & 62 & & & & & & \\
\hline & & 50 & & & & & & \\
\hline & & 56 & & & & & & \\
\hline & & $\mathbf{5 4 . 2}$ & & & & & & \\
\hline & & & & & & & & \\
\hline & & & & & & & & \\
\hline & & & & & & & & \\
\hline & & & & & & & \\
\hline \\
\hline
\end{tabular}




\begin{tabular}{|c|c|c|c|c|c|c|c|c|}
\hline & & 41 & & & 20 & & 0.15 & \\
\hline & & 58 & & & 10 & & 0.1 & \\
\hline & & 52 & & & 5 & & 1.2 & \\
\hline & & 52 & & & 10 & & 1.5 & \\
\hline & & 54 & & & 1 & & 0.45 & \\
\hline & & 43 & & & 50 & & 6 & \\
\hline & & 52.4 & & & 40 & & 0.3 & \\
\hline & & & & & 100 & & 1.2 & \\
\hline & & & & & 35.41 & & 1.5 & \\
\hline & & & & & 20 & & 0.2 & \\
\hline & & & & & $r=2$ & & 0.85 & \\
\hline & & & & & & & 0.45 & \\
\hline & & & & & & & $r=21$ & \\
\hline $\begin{array}{l}\text { Waypoint } \\
152\end{array}$ & & & & & & & & \\
\hline $\begin{array}{l}37.07517 \\
118.91678\end{array}$ & 2205 & 54 & -90 & $2(1)$ & 2 & 165 & 0.6 & $r=5$ \\
\hline & & 60 & & $>10(\mathrm{~F} 1)$ & 100 & 90,88 & 0.15 & \\
\hline & & 51 & & $>10(\mathrm{~V} 1)$ & 75 & 250 & 0.1 & \\
\hline & & 48 & & $4.5(\mathrm{~V} 2)$ & 200 & 180 & 0.1 & \\
\hline & & 62 & & $>5(\mathrm{~V} 3)$ & 25 & 220 & 0.1 & \\
\hline & & 51 & & & $r=2$ & $r=9$ & 0.3 & \\
\hline & & 56 & & & & & 2.1 & \\
\hline & & 43 & & & & & 0.3 & \\
\hline & & 54 & & & & & 2.4 & \\
\hline & & 58 & & & & & 1.2 & \\
\hline & & 48 & & & & & 1 & \\
\hline & & $\sqrt[52]{ }$ & & & & & 1.5 & \\
\hline & & 53 & & & & & 0.9 & \\
\hline & & 60 & & & & & 0.3 & \\
\hline & & 46 & & & & & 0.2 & \\
\hline & & 59 & & & & & 0.75 & \\
\hline & & \begin{tabular}{|l|l|}
53.4 \\
\end{tabular} & & & & & 0.3 & \\
\hline & & & & & & & $r=15$ & \\
\hline & & & & & & & & \\
\hline & & & & & & & & \\
\hline & & & & & & & & \\
\hline & & & & & & & & \\
\hline & & & & & & & & \\
\hline
\end{tabular}




\begin{tabular}{|c|c|c|c|c|c|c|c|c|}
\hline \multicolumn{9}{|l|}{\begin{tabular}{|l} 
Waypoint \\
153
\end{tabular}} \\
\hline $\begin{array}{l}37.07484 \\
118.91695\end{array}$ & 2231 & 54 & -45 & $>4(1)$ & 1 & 350,80 & 1.2 & $r=6$ \\
\hline & & 62 & & $1(2)$ & 10 & 80,90 & & \\
\hline & & 41 & & $1.5(\mathrm{~F} 1)$ & 1 & 210 & 1.5 & \\
\hline & & 53 & & $2(\mathrm{~F} 2)$ & 3 & 50 & 0.2 & \\
\hline & & 60 & & $2.5(\mathrm{~F} 3)$ & 20 & 155 & & \\
\hline & & 54 & & & 20 & $r=9$ & & \\
\hline & & 42 & & & 0.5 & & 0.6 & \\
\hline & & 63 & & & 0.5 & & 0.5 & \\
\hline & & 55 & & & 1 & & 1 & \\
\hline & & 46 & & & 20 & & 0.9 & \\
\hline & & 64 & & & 10 & & 0.84 & \\
\hline & & 44 & & & 7.91 & & 0.9 & \\
\hline & & 62 & & & 3 & & $r=21$ & \\
\hline & & 56 & & & $r=5$ & & & \\
\hline & & 60 & & & & & & \\
\hline & & 56 & & & & & & \\
\hline & & 66 & & & & & & \\
\hline & & 55.2 & & & & & & \\
\hline & & & & & & & & \\
\hline $\begin{array}{l}\text { Waypoint } \\
154\end{array}$ & & & & & & & & \\
\hline $\begin{array}{l}37.08761 \\
118.87350\end{array}$ & 2410 & 50 & -90 & $1(1)$ & face & 60,86 & 0.75 & $r=5$ \\
\hline & & 60 & & $4.5(2)$ & 200 & 280 & 1 & \\
\hline & & 53 & & 4.5 (Flak & 75 & 200 & 1.4 & \\
\hline & & 60 & & 1 (Flake & 1 & 200,90 & 3 & \\
\hline & & 50 & & 2 (Flake & 3 & 15 & 2.5 & \\
\hline & & 58 & & & 69.75 & $r=9$ & 1.2 & \\
\hline & & 63 & & & 39 & & 1.64 & \\
\hline & & 58 & & & $r=2$ & & 1.4 & \\
\hline & & 44 & & & & & $r=28$ & \\
\hline & & 60 & & & & & & \\
\hline & & 52 & & & & & & \\
\hline & & 53 & & & & & & \\
\hline & & 60 & & & & & & \\
\hline & & 61 & & & & & & \\
\hline & & 61 & & & & & & \\
\hline
\end{tabular}




\begin{tabular}{|l|l|l|l|l|l|l|l|l|}
\hline & & 50 & & & & & & \\
\hline & & 52 & & & & & & \\
\hline & & $\mathbf{5 5 . 6}$ & & & & & & \\
\hline & & & & & & & & \\
\hline $\begin{array}{l}\text { Waypoint } \\
155\end{array}$ & & & & & & & & \\
\hline $\begin{array}{l}37.08686 \\
118.87364\end{array}$ & 2459 & $70(\mathrm{P})$ & -90 & $1.75(1)$ & 1 & 215 & 0.45 & $r=6$ \\
\hline & & 70 & & $>5(2)$ & 1 & 220 & 0.45 & \\
\hline & & 70 & & $1(3)$ & 2 & 245 & 0.3 & \\
\hline & & 72 & & $>5(\mathrm{~V} 1)$ & 1000 & 210 & 0.6 & \\
\hline & & 68 & & & $\mathbf{2 5 1}$ & $\boldsymbol{r}=\mathbf{1 4}$ & $\mathbf{0 . 4 5}$ & \\
\hline & & 62 & & & $\mathbf{1 . 5}$ & & $\mathbf{0 . 4 5}$ & \\
\hline & & 65 & & & $\boldsymbol{r}=5$ & & $r=21$ & \\
\hline & & 64 & & & & & & \\
\hline & & 70 & & & & & & \\
\hline & & 71 & & & & & & \\
\hline & & 69 & & & & & & \\
\hline & & 64 & & & & & & \\
\hline & & 64 & & & & & & \\
\hline & & 66 & & & & & & \\
\hline & & 62 & & & & & & \\
\hline & & 62 & & & & & & \\
\hline & & 64 & & & & & & \\
\hline & & $\mathbf{6 6 . 6}$ & & & & & & \\
\hline & & & & & & & & \\
\hline & & & & & & & & \\
\hline & & & & & & & & \\
\hline \\
\hline
\end{tabular}




\begin{tabular}{|c|c|c|c|c|c|c|c|c|}
\hline & & 69 & & & & & 0.3 & \\
\hline & & 72 & & & & & 0.6 & \\
\hline & & 70 & & & & & 5 & \\
\hline & & 63 & & & & & 1.2 & \\
\hline & & 61 & & & & & 0.3 & \\
\hline & & 68 & & & & & 1.8 & \\
\hline & & 60.8 & & & & & 3 & \\
\hline & & & & & & & 1.26 & \\
\hline & & & & & & & 0.83 & \\
\hline & & & & & & & $r=21$ & \\
\hline & & & & & & & & \\
\hline $\begin{array}{l}\text { Waypoint } \\
157\end{array}$ & & & & & & & & \\
\hline $\begin{array}{l}37.08514 \\
118.87444\end{array}$ & 2503 & 64 & -45 & $4(1)$ & 1 & 115 & 1.8 & $r=6$ \\
\hline & & 58 & & $1.5(2)$ & 0.5 & 205 & 1.5 & \\
\hline & & 56 & & $1.5(3)$ & na & 175 & 0.6 & \\
\hline & & 74 & & $3(4)$ & na & 245 & 0.6 & \\
\hline & & 66 & & 7 (V1) & 305 & 305 & 0.6 & \\
\hline & & 52 & & $0.5(\mathrm{~V} 2)$ & 75 & 200 & 4.5 & \\
\hline & & 46 & & & $r=6$ & $r=20$ & 1.5 & \\
\hline & & 70 & & & & & 1.8 & \\
\hline & & 66 & & & & & 0.3 & \\
\hline & & 62 & & & & & 1.2 & \\
\hline & & 49 & & & & & 1.44 & \\
\hline & & 56 & & & & & 1.35 & \\
\hline & & 56 & & & & & $r=28$ & \\
\hline & & 62 & & & & & & \\
\hline & & 58 & & & & & & \\
\hline & & 70 & & & & & & \\
\hline & & 58 & & & & & & \\
\hline & & 60.2 & & & & & & \\
\hline & & & & & & & & \\
\hline & & & & & & & & \\
\hline & & & & & & & & \\
\hline & & & & & & & & \\
\hline & & & & & & & & \\
\hline & & & & & & & & \\
\hline & & & & & & & & \\
\hline
\end{tabular}




\begin{tabular}{|c|c|c|c|c|c|c|c|c|}
\hline \multicolumn{9}{|l|}{$\begin{array}{l}\text { Waypoint } \\
158\end{array}$} \\
\hline $\begin{array}{l}37.08435 \\
118.87475\end{array}$ & 2536 & 42 & -90 & $>4$ (1) & 20 & 200 & $1-3 m$ & $r=6$ \\
\hline & & 60 & & $>4(2)$ & 10 & 95,90 & $r=28$ & \\
\hline & & 58 & & $1(3)$ & 0.01 & 150 & & \\
\hline & & 48 & & $5(4)$ & 0.5 & 95 & & \\
\hline & & 59 & & & 30 & $r=18$ & & \\
\hline & & 54 & & & 15 & & & \\
\hline & & 55 & & & 12.59 & & & \\
\hline & & 58 & & & 12.5 & & & \\
\hline & & 56 & & & $r=5$ & & & \\
\hline & & 54 & & & & & & \\
\hline & & 53 & & & & & & \\
\hline & & 44 & & & & & & \\
\hline & & 57 & & & & & & \\
\hline & & 56 & & & & & & \\
\hline & & 56 & & & & & & \\
\hline & & 53 & & & & & & \\
\hline & & 58 & & & & & & \\
\hline & & 54.2 & & & & & & \\
\hline & & & & & & & & \\
\hline $\begin{array}{l}\text { Waypoint } \\
159 \text { (K6) }\end{array}$ & & & & & & & & \\
\hline $\begin{array}{l}37.08803 \\
118.87320\end{array}$ & 2453 & 60 & -90 & $>4$ (Flake & 75 & 115 & 0.15 & $r=5$ \\
\hline & & 52 & & 2.5 (Flak & 10 & 145 & 0.1 & \\
\hline & & 44 & & 1 (Flake & 10 & 65 & 0.2 & \\
\hline & & 58 & & $1.5(1)$ & 50 & 240,86 & 0.3 & \\
\hline & & 66 & & $1(2)$ & 50 & 115,90 & 0.3 & \\
\hline & & 56 & & $1.3(3)$ & 25 & 125,90 & 0.15 & \\
\hline & & 46 & & $1(4)$ & 15 & 195,75 & 0.3 & \\
\hline & & 65 & & & 100 & $r=14$ & 0.25 & \\
\hline & & 60 & & & 60 & & 0.2 & \\
\hline & & 5 & & & 20 & & 0.25 & \\
\hline & & 50 & & & 10 & & 0.4 & \\
\hline & & 58 & & & 60 & & 0.01 & \\
\hline & & 62 & & & $r=2$ & & 0.9 & \\
\hline & & 55 & & & & & 0.75 & \\
\hline & & 48 & & & & & 0.30 & \\
\hline
\end{tabular}




\begin{tabular}{|l|l|l|l|l|l|l|l|l|}
\hline & & 60 & & & & & $\mathbf{0 . 2 5}$ & \\
\hline & & 57 & & & & & $\boldsymbol{r}=\mathbf{1 5}$ & \\
\hline & & $\mathbf{5 6 . 2}$ & & & & & & \\
\hline & & & & & & & & \\
\hline $\begin{array}{l}\text { Waypoint } \\
160\end{array}$ & & & & & & & & \\
\hline 37.08861 & & & & & & & & \\
\hline 118.87283 & 2464 & 59 & -90 & $3-4$ (Set 1 & 10 & 20 & 0.3 & $r=6$ \\
\hline & & 62 & & $>3.5(2)$ & 10 & 285,90 & 0.4 & \\
\hline & & 47 & & 2 (Flake & 2 & 315 & 0.2 & \\
\hline & & 58 & & & 200 & $r=9$ & 4.5 & \\
\hline & & 61 & & & 100 & & 1.7 & \\
\hline & & 64 & & & 200 & & 2.4 & \\
\hline & & 56 & & & 50 & & 1 & \\
\hline & & 49 & & & $\mathbf{8 1 . 7 1 4}$ & & $\mathbf{1 . 5}$ & \\
\hline & & 54 & & & $\mathbf{5 0}$ & & $\mathbf{1}$ & \\
\hline & & 60 & & & $\boldsymbol{r}=\mathbf{2}$ & & $\boldsymbol{r}=\mathbf{2 8}$ & \\
\hline & & 51 & & & & & & \\
\hline & & 57 & & & & & & \\
\hline & & 46 & & & & & & \\
\hline & & 56 & & & & & & \\
\hline & & 56 & & & & & & \\
\hline & & 63 & & & & & & \\
\hline & & 60 & & & & & & \\
\hline & & $\mathbf{5 6 . 4}$ & & & & & & \\
\hline & & & & & & & & \\
\hline & & & & & & & & \\
\hline & & & & & & & & \\
\hline \\
\hline
\end{tabular}




\begin{tabular}{|l|l|l|l|l|l|l|l|l|}
\hline & & 52 & & & 5 & & 0.3 & \\
\hline & & 56 & & & 3 & & 0.45 & \\
\hline & & 56 & & & 1 & & 0.3 & \\
\hline & & 60 & & & 2 & & 0.45 & \\
\hline & & 58 & & & 10 & & 1.2 & \\
\hline & & 55 & & & 100 & & 1.5 & \\
\hline & & $\mathbf{5 4 . 1}$ & & & $\mathbf{4 8 . 4 7}$ & & 2 & \\
\hline & & & & & 10 & & $\mathbf{0 . 6 5}$ & \\
\hline & & & & & & & $\mathbf{0 . 4 5}$ & \\
\hline & & & & & & & & \\
\hline $\begin{array}{l}\text { Waypoint } \\
162\end{array}$ & & & & & & & & \\
\hline 37.09029 \\
118.87216 & 2515 & 63 & -90 & $\sim 3(1)$ & na & 15 & $>3 \mathrm{~m}$ & $r=6$ \\
\hline & & 70 & & $3.5(\mathrm{~V} 1)$ & 150 & 345 & $\mathbf{r} 30$ & \\
\hline & & 58 & & $3(\mathrm{~V} 2)$ & 40 & 260 & & \\
\hline & & 71 & & & $r=7$ & $r=20$ & & \\
\hline & & 68 & & & & & & \\
\hline & & 64 & & & & & & \\
\hline & & 61 & & & & & & \\
\hline & & 70 & & & & & & \\
\hline & & 68 & & & & & & \\
\hline & & 64 & & & & & & \\
\hline & & 64 & & & & & & \\
\hline & & 72 & & & & & & \\
\hline & & 72 & & & & & & \\
\hline & & 72 & & & & & & \\
\hline & & 68 & & & & & & \\
\hline & & 74 & & & & & & \\
\hline & & $\mathbf{6 3}$ & & & & & & \\
\hline & & & & & & & & \\
\hline & & & & & & & & \\
\hline & & & & & & & & \\
\hline & & & & & & & & \\
\hline & & & & & & & & \\
\hline
\end{tabular}




\begin{tabular}{|c|c|c|c|c|c|c|c|c|}
\hline \multicolumn{9}{|l|}{$\begin{array}{l}\text { Waypoint } \\
163\end{array}$} \\
\hline $\begin{array}{l}37.09109 \\
118.87156\end{array}$ & 2541 & 49 & -90 & $>10(1)$ & 10 & 185,84 & $1-3 m$ & $r=6$ \\
\hline & & 60 & & 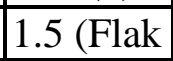 & 15 & 70 & $r=28$ & \\
\hline & & 58 & & 2 (Flake & 20 & $\sqrt[50]{50}$ & & \\
\hline & & 55 & & 1 (Flake & 20 & 50 & & \\
\hline & & 55 & & $>15(\mathrm{~V} 1)$ & 100 & 355 & & \\
\hline & & 56 & & & $r=4$ & $r=18$ & & \\
\hline & & 44 & & & & & & \\
\hline & & 62 & & & & & & \\
\hline & & 64 & & & & & & \\
\hline & & 58 & & & & & & \\
\hline & & 48 & & & & & & \\
\hline & & 60 & & & & & & \\
\hline & & 56 & & & & & & \\
\hline & & 56 & & & & & & \\
\hline & & 48 & & & & & & \\
\hline & & 55 & & & & & & \\
\hline & & 54 & & & & & & \\
\hline & & 55.2 & & & & & & \\
\hline & & & & & & & & \\
\hline $\begin{array}{l}\text { Waypoint } \\
164 \text { (K7) }\end{array}$ & & & & & & & & \\
\hline $\begin{array}{l}37.10564 \\
118.89648\end{array}$ & 2451 & 52 & -90 & $3(1)$ & 75 & 155,80 & $1-3 m$ & $r=5$ \\
\hline & & $\overline{52}$ & & $4(2)$ & 75 & 230 & $r=21$ & \\
\hline & & 59 & & $1.8(3)$ & 100 & 120,75 & & \\
\hline & & $\overline{54}$ & & $>4(4)$ & 50 & 260 & & \\
\hline & & 58 & & $>3(5)$ & 100 & 240,90 & & \\
\hline & & 56 & & $1(6)$ & 100 & 280,86 & & \\
\hline & & 64 & & $1.5(7)$ & 100 & 275,90 & & \\
\hline & & 62 & & $1.5(8)$ & 250 & 250,90 & & \\
\hline & & 50 & & $1.5(9)$ & 250 & 335,65 & & \\
\hline & & 55 & & & $r=2$ & $r=14$ & & \\
\hline & & 50 & & & & & & \\
\hline & & 58 & & & & & & \\
\hline & & 62 & & & & & & \\
\hline & & 54 & & & & & & \\
\hline & & 61 & & & & & & \\
\hline
\end{tabular}




\begin{tabular}{|l|l|l|l|l|l|l|l|l|}
\hline & & 52 & & & & & & \\
\hline & & 60 & & & & & & \\
\hline & & $\mathbf{5 6 . 4}$ & & & & & & \\
\hline & & & & & & & & \\
\hline $\begin{array}{l}\text { Waypoint } \\
165\end{array}$ & & & & & & & & \\
\hline $\begin{array}{l}37.10569 \\
118.89684\end{array}$ & 2472 & 60 & -90 & $1(\mathrm{~V} 1)$ & 130 & 275 & 0.2 & $\boldsymbol{r}=\mathbf{5}$ \\
\hline & & 52 & & $>3($ Set 1 & 180 & 275 & 0.3 & \\
\hline & & 48 & & $0.5($ Set 2 & 50 & 345,80 & 0.2 & \\
\hline & & 58 & & $4(3)$ & 25 & 260,90 & 0.6 & \\
\hline & & 51 & & & $\boldsymbol{r}=\mathbf{2}$ & $\mathbf{r}=\mathbf{1 4}$ & 0.15 & \\
\hline & & 56 & & & & & 0.15 & \\
\hline & & 52 & & & & & 0.45 & \\
\hline & & 56 & & & & & 1.2 & \\
\hline & & 61 & & & & & 0.9 & \\
\hline & & 62 & & & & & 1.5 & \\
\hline & & 56 & & & & & 0.6 & \\
\hline & & 50 & & & & & 0.5 & \\
\hline & & 50 & & & & & 0.6 & \\
\hline & & 60 & & & & & 0.45 & \\
\hline & & 54 & & & & & 0.5 & \\
\hline & & 62 & & & & & 0.6 & \\
\hline & & 62 & & & & & 0.3 & \\
\hline & & $\mathbf{5 5 . 9}$ & & & & & 0.6 & \\
\hline & & & & & & & $\mathbf{0 . 5 4}$ & \\
\hline & & & & & & & $\mathbf{0 . 5}$ & \\
\hline & & & & & & $\boldsymbol{r}=\mathbf{2 1}$ & \\
\hline
\end{tabular}




\begin{tabular}{|c|c|c|c|c|c|c|c|c|}
\hline \multicolumn{9}{|c|}{ Deadman Canyon } \\
\hline \multicolumn{9}{|c|}{\begin{tabular}{l|l|l|} 
Waypoint & \\
114 (D5) & \\
\end{tabular}} \\
\hline Lat/Long & $\begin{array}{l}\text { Elev. } \\
(\mathrm{m})\end{array}$ & $\mathrm{SH}$ & Alpha & $\begin{array}{l}\text { Joint } \\
\text { length } \\
(\mathrm{m})\end{array}$ & $\begin{array}{l}\text { Joint } \\
\text { width } \\
(\mathrm{mm})\end{array}$ & $\begin{array}{l}\text { Spacing } \\
\text { of joints } \\
\text { (m) }\end{array}$ & $\begin{array}{l}\text { Joint strike } \\
\text { and dip }\end{array}$ & Continuity \\
\hline $\begin{array}{l}36.68356 \\
118.57772\end{array}$ & 2404 & 35 & -90 & $2(1)$ & 10 & 0.30 & $320(?), 52$ & $r=5$ \\
\hline & & 45 & & $1.2(2)$ & 9 & 0.50 & 330,60 & \\
\hline & & 36 & & $0.6(3)$ & 10 & 0.60 & 320,75 & \\
\hline & & 48 & & $1(4)$ & 2 & 1.00 & 175 & \\
\hline & & 30 & & $1(5)$ & 3 & 0.60 & 170 & \\
\hline & & 38 & & $2.5(6)$ & 2 & 0.55 & 175 & \\
\hline & & 44 & & $0.3(7)$ & 2.5 & $r=21$ & 310,85 & \\
\hline & & 60 & & $0.6(8)$ & 1 & & 330 & \\
\hline & & 42 & & $1.5(9)$ & 3 & & 330,75 & \\
\hline & & 58 & & $2(10)$ & 2.5 & & 155 & \\
\hline & & 52 & & $3(11)$ & 18 & & 340,80 & \\
\hline & & 37 & & $\begin{array}{l}>1.5 \\
(12)\end{array}$ & 3 & & 200,50 & \\
\hline & & 44 & & $0.5(13)$ & 1.5 & & $r=9$ & \\
\hline & & 46 & & $0.5(14)$ & 1 & & & \\
\hline & & 56 & & & 4.89 & & & \\
\hline & & 34 & & & 2.75 & & & \\
\hline & & 52 & & & $r=2$ & & & \\
\hline & & 45 & & & & & & \\
\hline & & & & & & & & \\
\hline $\begin{array}{l}\text { Waypoint } \\
115\end{array}$ & & & & & & & & \\
\hline $\begin{array}{l}36.68367 \\
118.57719 \\
\end{array}$ & 2488 & 36 & -90 & $3(1)$ & 1 & 0.20 & 300,85 & $r=6$ \\
\hline & & 42 & & $1.8(2)$ & na & 1.00 & 160 & \\
\hline & & 42 & & $2(3)$ & na & 1.20 & 220 & \\
\hline & & 50 & & $1(4)$ & na & 0.30 & 140 & \\
\hline & & 46 & & $3.5(5)$ & face & 0.50 & 325,10 & \\
\hline & & 66 & & $\begin{array}{l}>4(6) \\
(\text { Set }) \\
2.5 \& 2\end{array}$ & 2.25 & 0.15 & 220,70 & \\
\hline
\end{tabular}




\begin{tabular}{|c|c|c|c|c|c|c|c|c|}
\hline & & 43 & & $\begin{array}{l}1(7) \\
\text { (Set) } \\
\text { All 1m. }\end{array}$ & 2 & 0.40 & 130 & \\
\hline & & 48 & & $1.5(8)$ & 2.5 & $r=21$ & 230 & \\
\hline & & 49 & & $4(9)$ & 5 & & 350 & \\
\hline & & 60 & & & 2.55 & & $r=20$ & \\
\hline & & 40 & & & 2.25 & & & \\
\hline & & 52 & & & $r=6$ & & & \\
\hline & & 44 & & & & & & \\
\hline & & 62 & & & & & & \\
\hline & & 38 & & & & & & \\
\hline & & 48 & & & & & & \\
\hline & & 44 & & & & & & \\
\hline & & 48 & & & & & & \\
\hline & & & & & & & & \\
\hline $\begin{array}{l}\text { Waypoint } \\
116\end{array}$ & & & & & & & & \\
\hline $\begin{array}{l}36.68356 \\
118.57685 \\
\end{array}$ & 2499 & 42 & -45 & $\begin{array}{l}3.5 \text { (Set } \\
1)\end{array}$ & $2-0.1$ & 0.76 & 75,5 & $r=6$ \\
\hline & & 60 & & $\begin{array}{l}3 \text { (Set } \\
2)\end{array}$ & $0.2-0.1$ & 0.15 & 130,35 & \\
\hline & & 45 & & $\begin{array}{l}1.5(\mathrm{Set} \\
3)\end{array}$ & $0.4-0.1$ & 0.20 & 90,70 & \\
\hline & & 43 & & & 0.86 & 0.07 & $r=14$ & \\
\hline & & 61 & & & $r=4$ & 0.60 & & \\
\hline & & 62 & & & & 1.20 & & \\
\hline & & 62 & & & & 0.02 & & \\
\hline & & 44 & & & & 0.20 & & \\
\hline & & 43 & & & & $r=21$ & & \\
\hline & & 38 & & & & & & \\
\hline & & 48 & & & & & & \\
\hline & & 39 & & & & & & \\
\hline & & 45 & & & & & & \\
\hline & & 46 & & & & & & \\
\hline & & 41 & & & & & & \\
\hline & & 48 & & & & & & \\
\hline & & 30 & & & & & & \\
\hline & & 47 & & & & & & \\
\hline & & & & & & & & \\
\hline
\end{tabular}




\begin{tabular}{|c|c|c|c|c|c|c|c|c|}
\hline \multicolumn{9}{|l|}{$\begin{array}{l}\text { Waypoint } \\
117 \text { (D1) }\end{array}$} \\
\hline $\begin{array}{l}36.64120 \\
118.58739\end{array}$ & 2693 & 46 & -90 & $2(1)$ & 5 & 1.40 & 200 & $r=5$ \\
\hline & & 34 & & $4(2)$ & 10 & 0.50 & 175 & \\
\hline & & 40 & & $\begin{array}{l}1 \text { (3) } \\
\text { (Set) }\end{array}$ & 12 & 0.10 & 285,85 & \\
\hline & & 44 & & $3.5(4)$ & 5 & 0.15 & $\begin{array}{ll}185 \\
\end{array}$ & \\
\hline & & 35 & & $>5(5)$ & $\begin{array}{l}\text { top } \\
\text { edge }\end{array}$ & 0.30 & 170 & \\
\hline & & 52 & & & 8 & 0.60 & $r=9$ & \\
\hline & & 40 & & & 7.5 & 1.80 & & \\
\hline & & 54 & & & $r=5$ & 0.69 & & \\
\hline & & 46 & & & & 0.50 & & \\
\hline & & 42 & & & & $r=21$ & & \\
\hline & & 50 & & & & & & \\
\hline & & 62 & & & & & & \\
\hline & & 52 & & & & & & \\
\hline & & 41 & & & & & & \\
\hline & & 43 & & & & & & \\
\hline & & 55 & & & & & & \\
\hline & & 38 & & & & & & \\
\hline & & 46 & & & & & & \\
\hline & & & & & & & & \\
\hline $\begin{array}{l}\text { Waypoint } \\
118\end{array}$ & & & & & & & & \\
\hline $\begin{array}{l}36.64114 \\
118.58816\end{array}$ & 2724 & 42 & -90 & $\begin{array}{l}2-2.5 \\
\text { (Set } 1)\end{array}$ & 4 & 1.80 & 195 & $r=6$ \\
\hline & & 46 & & $\begin{array}{l}1 \text { (Set } \\
2 \text { ) }\end{array}$ & 3 & 0.30 & 120,20 & \\
\hline & & 53 & & $3(3)$ & 5 & 0.70 & 155 & \\
\hline & & 43 & & $4(4)$ & 4 & 2.50 & 25 & \\
\hline & & 37 & & $\begin{array}{l}1-1.5 \\
(5)(\mathrm{Set})\end{array}$ & 2 & 1.00 & 90,15 & \\
\hline & & 34 & & $\begin{array}{l}15 .-2 \\
(6)\end{array}$ & 1 & 0.10 & 25,10 & \\
\hline & & 43 & & $2(7)$ & 2 & 0.60 & 235 & \\
\hline & & 32 & & $1(8)$ & 1 & 0.02 & 315 & \\
\hline & & 43 & & & 2.75 & 0.15 & $r=9$ & \\
\hline
\end{tabular}




\begin{tabular}{|c|c|c|c|c|c|c|c|c|}
\hline & & 52 & & & 2.5 & 0.20 & & \\
\hline & & 56 & & & $r=5$ & 0.45 & & \\
\hline & & 42 & & & & $r=21$ & & \\
\hline & & 40 & & & & & & \\
\hline & & 54 & & & & & & \\
\hline & & 50 & & & & & & \\
\hline & & 43 & & & & & & \\
\hline & & 41 & & & & & & \\
\hline & & 44 & & & & & & \\
\hline & & & & & & & & \\
\hline $\begin{array}{l}\text { Waypoint } \\
119\end{array}$ & & & & & & & & \\
\hline $\begin{array}{l}36.64113 \\
118.5887\end{array}$ & 2754 & 34 & -90 & $\begin{array}{l}>4 \text { (Set } \\
1)\end{array}$ & 1 & 0.25 & Strike into f & $r=6$ \\
\hline & & 42 & & \begin{tabular}{|l|}
0.6 (Set \\
2 )
\end{tabular} & 4 & 0.30 & 20 & \\
\hline & & 41 & & $\begin{array}{l}>5 \text { (Set } \\
3 \text { ) }\end{array}$ & $\begin{array}{ll}1 & \text { for } \\
\text { most. } \\
15 & \text { for } \\
\text { largest. }\end{array}$ & $\begin{array}{l}0.60 \\
0.0\end{array}$ & 280,65 & \\
\hline & & 39 & & \begin{tabular}{|l|}
$0.5-1$ \\
$(\operatorname{Set} 4)$
\end{tabular} & na & 0.30 & \multicolumn{2}{|c|}{ 05, dip into face } \\
\hline & & 50 & & & $r=2$ & 0.90 & $r=14$ & \\
\hline & & 34 & & & & 0.60 & & \\
\hline & & 33 & & & & 0.45 & & \\
\hline & & 45 & & & & $r=21$ & & \\
\hline & & 37 & & & & & & \\
\hline & & 45 & & & & & & \\
\hline & & 50 & & & & & & \\
\hline & & 36 & & & & & & \\
\hline & & 34 & & & & & & \\
\hline & & 52 & & & & & & \\
\hline & & 44 & & & & & & \\
\hline & & 41 & & & & & & \\
\hline & & 40 & & & & & & \\
\hline & & 41 & & & & & & \\
\hline & & 43 & & & & & & \\
\hline & & & & & & & & \\
\hline & & & & & & & & \\
\hline
\end{tabular}




\begin{tabular}{|c|c|c|c|c|c|c|c|c|}
\hline \multicolumn{9}{|l|}{\begin{tabular}{|l|} 
Waypoint \\
120 (D2)
\end{tabular}} \\
\hline $\begin{array}{l}36.66020 \\
118.58072\end{array}$ & 2672 & 35 & -90 & $\begin{array}{l}3.5 \text { (Set } \\
1)\end{array}$ & $2-4 \mathrm{~cm}$ & 0.60 & 270,65 & \\
\hline & & 62 & & $\begin{array}{l}>4 \text { (Set } \\
2)\end{array}$ & 15 & 0.05 & 45 & $r=6$ \\
\hline & & 50 & & & $r=4$ & 0.30 & $r=14$ & \\
\hline & & 61 & & & & 0.20 & & \\
\hline & & 46 & & & & 1.20 & & \\
\hline & & 44 & & & & 0.50 & & \\
\hline & & 48 & & & & 0.90 & & \\
\hline & & 50 & & & & 0.50 & & \\
\hline & & 58 & & & & $r=21$ & & \\
\hline & & 46 & & & & & & \\
\hline & & 48 & & & & & & \\
\hline & & 50 & & & & & & \\
\hline & & 54 & & & & & & \\
\hline & & 57 & & & & & & \\
\hline & & 54 & & & & & & \\
\hline & & 54 & & & & & & \\
\hline & & 54 & & & & & & \\
\hline & & 51 & & & & & & \\
\hline & & & & & & & & \\
\hline $\begin{array}{l}\text { Waypoint } \\
121\end{array}$ & & & & & & & & \\
\hline $\begin{array}{l}36.66047 \\
118.58121\end{array}$ & 2695 & 51 & -90 & $\begin{array}{l}\text { 1.8 (Set } \\
\text { 1) }\end{array}$ & 4 & 0.30 & 320,80 & $r=5$ \\
\hline & & 45 & & $2.5(2)$ & 5 & 0.20 & 190 & \\
\hline & & 53 & & 1 (3) & 1 & 0.90 & 270 & \\
\hline & & 52 & & $2(4)$ & 3.5 & 0.15 & 300,55 & \\
\hline & & 58 & & & $r=2$ & 0.60 & $r=14$ & \\
\hline & & 42 & & & & 0 & & \\
\hline & & 49 & & & & $\mathrm{r}=21$ & & \\
\hline & & 42 & & & & & & \\
\hline & & 44 & & & & & & \\
\hline & & 54 & & & & & & \\
\hline & & 48 & & & & & & \\
\hline & & 57 & & & & & & \\
\hline & & 47 & & & & & & \\
\hline & & 48 & & & & & & \\
\hline
\end{tabular}




\begin{tabular}{|c|c|c|c|c|c|c|c|c|}
\hline & & 57 & & & & & & \\
\hline & & 53 & & & & & & \\
\hline & & 44 & & & & & & \\
\hline & & 50 & & & & & & \\
\hline & & & & & & & & \\
\hline \multicolumn{9}{|l|}{$\begin{array}{l}\text { Waypoint } \\
122 \text { (D3) }\end{array}$} \\
\hline $\begin{array}{l}36.66129 \\
118.57693\end{array}$ & 2640 & $68(\mathrm{P})$ & -45 & $\begin{array}{l}\text { No } \\
\text { visible } \\
\text { joints. }\end{array}$ & $r=7$ & $r=30$ & $r=20$ & $r=7$ \\
\hline & & \begin{tabular}{|l|}
66 \\
\end{tabular} & & 0 & & & & \\
\hline & & 66 & & & & & & \\
\hline & & 65 & & & & & & \\
\hline & & 55 & & & & & & \\
\hline & & 66 & & & & & & \\
\hline & & 68 & & & & & & \\
\hline & & 64 & & & & & & \\
\hline & & 68 & & & & & & \\
\hline & & 62 & & & & & & \\
\hline & & 66 & & & & & & \\
\hline & & 69 & & & & & & \\
\hline & & 68 & & & & & & \\
\hline & & 68 & & & & & & \\
\hline & & 67 & & & & & & \\
\hline & & 58 & & & & & & \\
\hline & & 68 & & & & & & \\
\hline & & 61 & & & & & & \\
\hline & & & & & & & & \\
\hline $\begin{array}{l}\text { Waypoint } \\
123\end{array}$ & & & & & & & & \\
\hline $\begin{array}{l}36.66078 \\
118.57639\end{array}$ & 2665 & $68(\mathrm{P})$ & -45 & $>4(1)$ & 2.5 & 1.20 & 8,10 & $r=5$ \\
\hline & & 66 & & $0.7(2)$ & 10 & 1.90 & 115,20 & \\
\hline & & 66 & & $0.5(3)$ & 3 & 0.40 & 115,30 & \\
\hline & & 65 & & $1.5(4)$ & 2.5 & 0.30 & 10 & \\
\hline & & 55 & & $>3(5)$ & 8 & 0.95 & 5,15 & \\
\hline & & 66 & & $0.3(6)$ & na & \begin{tabular}{|c|}
0.80 \\
\end{tabular} & & \\
\hline & & 68 & & $3.5(7)$ & 2 & $r=21$ & 5 & \\
\hline & & 64 & & $3(8)$ & 2 & & 7 & \\
\hline & & 68 & & $0.4(9)$ & 7.5 & & 115,30 & \\
\hline
\end{tabular}




\begin{tabular}{|c|c|c|c|c|c|c|c|c|}
\hline & & 62 & & $2.5(10)$ & 2 & & 6 & \\
\hline & & 66 & & $1(11)$ & na & & 160 & \\
\hline & & 69 & & $2(12)$ & na & & 160 & \\
\hline & & 68 & & & $r=5$ & & $r=14$ & \\
\hline & & 68 & & & & & & \\
\hline & & 67 & & & & & & \\
\hline & & 58 & & & & & & \\
\hline & & 68 & & & & & & \\
\hline & & 61 & & & & & & \\
\hline & & & & & & & & \\
\hline $\begin{array}{l}\text { Waypoint } \\
124\end{array}$ & & & & & & & & \\
\hline $\begin{array}{l}36.66010 \\
118.57553\end{array}$ & 2725 & 58 & -90 & $2(1)$ & 1 & 0.30 & 120,30 & $r=6$ \\
\hline & & 52 & & $3.5(2)$ & 1.5 & 0.90 & 100,35 & \\
\hline & & 60 & & $3(3)$ & 1 & 1.50 & 200 & \\
\hline & & 57 & & $>4(4)$ & 1 & 0.25 & 200 & \\
\hline & & 56 & & $>4(5)$ & 2 & 0.30 & 245 & \\
\hline & & 64 & & $3.5(6)$ & $\begin{array}{l}12.5 \\
\text { (flake) }\end{array}$ & 0.15 & 140 & \\
\hline & & 58 & & $1.5(7)$ & 1.5 & 0.80 & 140 & \\
\hline & & 58 & & $1(8)$ & 2.5 & 2.00 & 265 & \\
\hline & & 60 & & $>4(9)$ & 15 & 0.55 & 175,25 & \\
\hline & & 64 & & $>5(10)$ & 10 & $r=21$ & 250 & \\
\hline & & 58 & & $1.5(11)$ & 1 & & 140,20 & \\
\hline & & 53 & & $1.5(12)$ & 3 & & 200 & \\
\hline & & 59 & & $>3(13)$ & 2 & & & \\
\hline & & 59 & & $>4(14)$ & 2 & & 260 & \\
\hline & & 66 & & & 3 & & $r=14$ & \\
\hline & & 66 & & & $r=5$ & & & \\
\hline & & 62 & & & & & & \\
\hline & & 59 & & & & & & \\
\hline & & & & & & & & \\
\hline & & & & & & & & \\
\hline & & & & & & & & \\
\hline & & & & & & & & \\
\hline
\end{tabular}




\begin{tabular}{|c|c|c|c|c|c|c|c|c|}
\hline \begin{tabular}{|l|} 
Waypoint \\
125 (D4)
\end{tabular} & & & & & & & & \\
\hline \begin{tabular}{|l|}
36.6819 \\
118.57624
\end{tabular} & 2515 & 43 & -90 & $\begin{array}{l}1.5-2.5 \\
\text { (Set } 1)\end{array}$ & 9 & 0.60 & 60,50 & $r=6$ \\
\hline & & 60 & & $\begin{array}{l}1-1.5 \\
\text { Set 2) }\end{array}$ & 0.5 & 1.20 & 300,80 & \\
\hline & & 47 & & $\begin{array}{l}0.3 \text { (Set } \\
3 \text { ) }\end{array}$ & 7.5 & 0.30 & 45 & \\
\hline & & 44 & & $\begin{array}{l}1.5(\mathrm{Set} \\
4)\end{array}$ & 15 & 1.50 & 60,75 & \\
\hline & & 41 & & $2.5(5)$ & 10 & 0.25 & 30,65 & \\
\hline & & 52 & & $0.6(6)$ & 7.5 & 0.60 & 120 & \\
\hline & & 44 & & & $r=4$ & $r=21$ & $r=18$ & \\
\hline & & 44 & & & & & & \\
\hline & & 48 & & & & & & \\
\hline & & 54 & & & & & & \\
\hline & & 48 & & & & & & \\
\hline & & 50 & & & & & & \\
\hline & & 47 & & & & & & \\
\hline & & 48 & & & & & & \\
\hline & & 50 & & & & & & \\
\hline & & 50 & & & & & & \\
\hline & & 50 & & & & & & \\
\hline & & 48 & & & & & & \\
\hline & & & & & & & & \\
\hline $\begin{array}{l}\text { Waypoint } \\
126\end{array}$ & & & & & & & & \\
\hline \begin{tabular}{|l|}
36.68018 \\
118.57571
\end{tabular} & 2549 & $54(\mathrm{P})$ & -90 & $\begin{array}{l}>4 \text { (Set } \\
1)\end{array}$ & $15-20$ & 0.60 & 77 & $r=5$ \\
\hline & & 62 & & $1(2)$ & 1 & 0.25 & 140 & \\
\hline & & 64 & & $>2(3)$ & 1 & 1.20 & 135 & \\
\hline & & 62 & & $2(4)$ & 9 & 0.30 & 140 & \\
\hline & & 64 & & $>5(5)$ & 7 & 1.80 & 140 & \\
\hline & & 52 & & \begin{tabular}{|l}
$\sim 5$ \\
(Flake \\
1 )
\end{tabular} & 13 & 0.60 & See Photos. & \\
\hline & & 68 & & & 6.2 & $r=21$ & $r=14$ & \\
\hline & & 66 & & & 7 & & & \\
\hline & & 68 & & & $r=5$ & & & \\
\hline & & 56 & & & & & & \\
\hline
\end{tabular}




\begin{tabular}{|c|c|c|c|c|c|c|c|c|}
\hline & & 65 & & & & & & \\
\hline & & 62 & & & & & & \\
\hline & & 70 & & & & & & \\
\hline & & 66 & & & & & & \\
\hline & & 66 & & & & & & \\
\hline & & 65 & & & & & & \\
\hline & & 64 & & & & & & \\
\hline & & \begin{tabular}{|l|}
57 \\
\end{tabular} & & & & & & \\
\hline & & & & & & & & \\
\hline $\begin{array}{l}\text { Waypoint } \\
127\end{array}$ & & & & & & & & \\
\hline $\begin{array}{l}36.68022 \\
118.57536\end{array}$ & 2579 & 70 & -90 & $\begin{array}{l}0.4-0.5 \\
\text { (Set 1) }\end{array}$ & 13 & 1.00 & 55 & $r=5$ \\
\hline & & 66 & & $\begin{array}{l}3-3.5 \\
\text { (Set 2) }\end{array}$ & $\begin{array}{l}10 \& \\
\text { na }\end{array}$ & 0.60 & 325,20 & \\
\hline & & 58 & & $\begin{array}{l}3(\mathrm{Set} \\
3)\end{array}$ & 1 to 2 & 1.50 & 135,30 & \\
\hline & & 64 & & $2(4)$ & 2.5 & 0.90 & 65,20 & \\
\hline & & 74 & & $4(5)$ & 7 & 0.95 & 130,80 & \\
\hline & & 68 & & $2(6)$ & 20 & $r=21$ & 60,70 & \\
\hline & & 63 & & $>4(7)$ & 4 & & 320 & \\
\hline & & 56 & & $0.5(8)$ & face & & 770,40 & \\
\hline & & 58 & & & $r=4$ & & $r=18$ & \\
\hline & & 63 & & & & & & \\
\hline & & 64 & & & & & & \\
\hline & & 67 & & & & & & \\
\hline & & 64 & & & & & & \\
\hline & & 58 & & & & & & \\
\hline & & 68 & & & & & & \\
\hline & & 69 & & & & & & \\
\hline & & 65 & & & & & & \\
\hline & & 64 & & & & & & \\
\hline
\end{tabular}

